\title{
Reactivity and Selectivity in the Wittig Reaction: A Computational Study
}

Raphä̈l Robiette, Jeffery Richardson, Varinder K. Aggarwal, * and Jeremy N. Harvey*

School of Chemistry, University of Bristol, Cantock's Close, Bristol BS8 1TS, U.K.

E-mail: jeremy.harvey@bristol.ac.uk; v.aggarwal@bristol.ac.uk

\section{Supporting information}

I. Gaussian 03 reference

II. Molpro reference

III. Model reaction results

IV. B3LYP/6-311+G**(THF) energies of TS1s

V. Ylide dipoles

VI. Energies and geometries 


\section{Gaussian 03 reference}

Gaussian 03, Revision B.04, Frisch, M. J.; Trucks, G. W.; Schlegel, H. B.; Scuseria, G. E.; Robb, M. A.; Cheeseman, J. R.; Montgomery, Jr., J. A.; Vreven, T.; Kudin, K. N.; Burant, J. C.; Millam, J. M.; Iyengar, S. S.; Tomasi, J.; Barone, V.; Mennucci, B.; Cossi, M.; Scalmani, G.; Rega, N.; Petersson, G. A.; Nakatsuji, H.; Hada, M.; Ehara, M.; Toyota, K.; Fukuda, R.; Hasegawa, J.; Ishida, M.; Nakajima, T.; Honda, Y.; Kitao, O.; Nakai, H.; Klene, M.; Li, X.; Knox, J. E.; Hratchian, H. P.; Cross, J. B.; Bakken, V.; Adamo, C.; Jaramillo, J.; Gomperts, R.; Stratmann, R. E.; Yazyev, O.; Austin, A. J.; Cammi, R.; Pomelli, C.; Ochterski, J. W.; Ayala, P. Y.; Morokuma, K.; Voth, G. A.; Salvador, P.; Dannenberg, J. J.; Zakrzewski, V. G.; Dapprich, S.; Daniels, A. D.; Strain, M. C.; Farkas, O.; Malick, D. K.; Rabuck, A. D.; Raghavachari, K.; Foresman, J. B.; Ortiz, J. V.; Cui, Q.; Baboul, A. G.; Clifford, S.; Cioslowski, J.; Stefanov, B. B.; Liu, G.; Liashenko, A.; Piskorz, P.; Komaromi, I.; Martin, R. L.; Fox, D. J.; Keith, T.; Al-Laham, M. A.; Peng, C. Y.; Nanayakkara, A.; Challacombe, M.; Gill, P. M. W.; Johnson, B.; Chen, W.; Wong, M. W.; Gonzalez, C.; and Pople, J. A.; Gaussian, Inc., Pittsburgh, 2003.

\section{MOLPRO reference}

MOLPRO, Version 2002.3, Werner H.-J; Knowles, P.J.; Schütz, M.; Lindh, R.; Celani, P.; Korona, T.; Rauhut, G.; Manby, F.R.; Amos, R.D.; Bernhardsson, A.; Berning, A.; Cooper, D.L.; Deegan, M.J.O.; Dobbyn, A.J.; Eckert, F.; Hampel, C.; Hetzer, G.; Lloyd, A.W.; McNicholas, S.J.; Meyer, W.; Mura, M.E.; Nicklaß, A.; Palmieri, P.; Pitzer, R.; Schumann, U.; Stoll, H.; Stone A.J.; Tarroni, R. and Thorsteinsson, $\mathrm{T}$.

\section{Model reaction results}

Relative energies (in $\mathrm{kcal} / \mathrm{mol}$ ) to the reactants of TSs and intermediates, and relevant geometrical parameters for TS1, of the reaction $\mathrm{CH}_{2} \mathrm{O}+\mathrm{CH}_{2} \mathrm{PMe}_{3} \rightarrow \mathrm{CH}_{2}=\mathrm{CH}_{2}+\mathrm{P}(\mathrm{O}) \mathrm{Me}_{3}$

\begin{tabular}{|c|c|c|c|c|c|c|c|c|}
\hline \multirow[b]{2}{*}{ Method } & \multirow[b]{2}{*}{$\begin{array}{c}\mathrm{vdw} \\
\text { complex }\end{array}$} & \multirow[b]{2}{*}{ TS1 } & \multirow[b]{2}{*}{ OP1 } & \multirow[b]{2}{*}{ OP2 } & \multirow[b]{2}{*}{ TS2 } & \multirow[b]{2}{*}{$\begin{array}{l}\mathrm{CH}_{2}=\mathrm{CH}_{2}+ \\
\mathrm{POMe}_{3}\end{array}$} & \multicolumn{2}{|c|}{ Structure of TS1 } \\
\hline & & & & & & & C-C & PCCO \\
\hline B3LYP/6-31G* & -9.35 & -8.84 & -30.94 & -29.14 & -10.13 & -54.84 & 2.048 & 1.0 \\
\hline B3LYP/6-31+G* & -7.10 & -6.61 & -27.22 & -23.76 & -6.21 & -53.08 & 2.133 & 10.3 \\
\hline B3LYP/6-311+G** & -7.08 & -5.94 & -23.49 & -19.44 & -3.75 & -51.62 & 2.120 & 0.9 \\
\hline MP2/6-31G* & -9.62 & -8.65 & -36.93 & -34.75 & -12.51 & -57.93 & 2.140 & 0.0 \\
\hline $\mathrm{MP} 2 / 6-31+\mathrm{G}^{*}$ & -8.12 & -7.24 & -34.45 & -30.32 & -10.39 & -55.84 & 2.205 & 0.1 \\
\hline $\mathrm{MP} 2 / 6-311+\mathrm{G}^{* *}$ & -8.37 & -6.28 & -29.82 & -26.00 & -6.14 & -48.91 & 2.098 & 0.1 \\
\hline B3LYP/6-311+G**// B3LYP/6-31G* & -6.54 & -6.01 & -23.13 & -19.31 & -3.62 & -51.71 & & \\
\hline MP2/6-311+G**// B3LYP/6-31G* & -7.06 & -6.35 & -29.12 & -26.03 & -6.08 & -49.59 & & \\
\hline QCISD(T)/6-31G*//B3LYP/6-31G* & -8.70 & -7.23 & -34.27 & -31.88 & -10.18 & -57.52 & & \\
\hline QCISD(T)/6-31G*//MP2/6-31G* & -8.90 & -7.10 & -34.21 & -31.75 & -9.94 & -57.41 & & \\
\hline G3(MP2)/B3LYP & -4.92 & -2.44 & -27.40 & -24.35 & -8.34 & -54.47 & & \\
\hline B3LYP/6-31G*(THF) & $-{ }^{\mathrm{a}}$ & $-{ }^{\mathrm{a}}$ & -27.08 & -23.50 & -5.66 & -57.05 & & \\
\hline B3LYP/6-31G*(THF)//B3LYP/6-31G* & -7.57 & -10.32 & -26.91 & -23.81 & -5.97 & -57.47 & & \\
\hline B3LYP/6-311+G**(THF)//B3LYP/6-31G* & -4.00 & -7.19 & -18.12 & -12.80 & 0.81 & -53.99 & & \\
\hline
\end{tabular}


${ }^{a}$ not found at this level of theory

\section{B3LYP/6-311+G**(THF) energies of TS1s}

\begin{tabular}{|c|c|c|c|}
\hline Reaction & & B3LYP/6-31G*(THF) ${ }^{a}$ & B3LYP/6-311+G**(THF) ${ }^{a}$ \\
\hline \multirow[t]{2}{*}{ (1) $\mathrm{Me}_{3} \mathrm{PCHMe}+\mathrm{MeCHO}$} & cis & -1.47 & 0.96 \\
\hline & trans & -1.48 & 1.10 \\
\hline \multirow[t]{2}{*}{ (2) $\mathrm{Ph}_{3} \mathrm{PCHMe}+\mathrm{MeCHO}$} & cis & -0.23 & 1.40 \\
\hline & trans & 1.96 & 4.95 \\
\hline \multirow[t]{2}{*}{ (3) $\mathrm{Me}_{3} \mathrm{PCHPh}+\mathrm{PhCHO}$} & cis & 7.68 & 9.28 \\
\hline & trans & 5.05 & 6.00 \\
\hline \multirow[t]{2}{*}{ (4) $\mathrm{Ph}_{3} \mathrm{PCHPh}+\mathrm{PhCHO}$} & cis & 11.98 & 13.23 \\
\hline & trans & 12.39 & 14.10 \\
\hline \multirow[t]{2}{*}{ (5) $(\mathrm{MeO})_{3} \mathrm{PCHPh}+\mathrm{PhCHO}$} & cis & 16.31 & 18.65 \\
\hline & trans & 13.26 & 15.65 \\
\hline \multirow[t]{2}{*}{ (6) $\mathrm{Me}_{3} \mathrm{PCHCO}_{2} \mathrm{Me}+\mathrm{PhCHO}$} & cis & 15.95 & 17.40 \\
\hline & trans & 12.29 & 14.78 \\
\hline \multirow[t]{2}{*}{ (7) $\mathrm{Ph}_{3} \mathrm{PCHCO}_{2} \mathrm{Me}+\mathrm{PhCHO}$} & cis & 23.76 & 26.70 \\
\hline & trans & 21.30 & 24.33 \\
\hline \multirow[t]{2}{*}{ (8) $(\mathrm{MeO})_{3} \mathrm{PCHCO}_{2} \mathrm{Me}+\mathrm{PhCHO}$} & cis & 21.26 & 25.17 \\
\hline & trans & 19.77 & 23.09 \\
\hline
\end{tabular}

${ }^{\mathrm{a}}$ Single point calculations at the B3LYP/6-31G*(THF) geometries 
V. Ylides dipoles

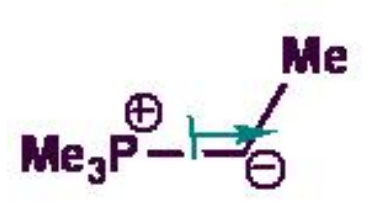

dipole $=\mathbf{2 . 8 2}$

x: 0.04

$y:-0.27$

z: 2.81

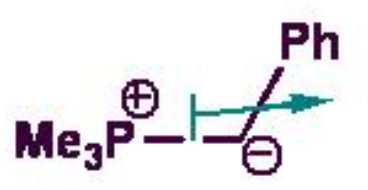

dipole $=6.02$

x: 0.00

$y:-1.85$

z: 5.71

$\mathrm{Me}_{3} \mathrm{P}-\overbrace{\theta}^{\text {CoOMe }}$

dipole $=4.60 \mathrm{D}$

x: 0.00

y: -2.65

z: 3.75

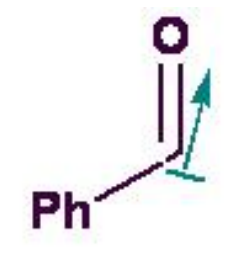

dipole $=\mathbf{3 . 3 7}$

x: 3.00

y: -1.52

z: 0.00

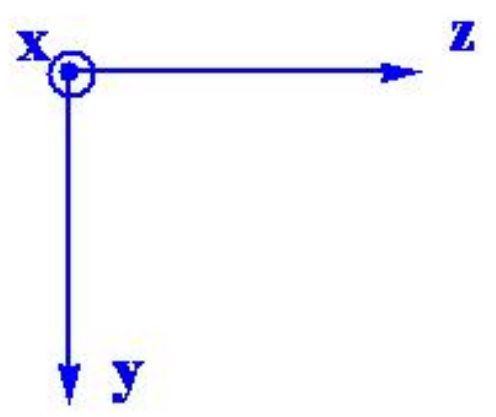

$\mathrm{Ph}_{3} \stackrel{\oplus}{\mathrm{P}}-\stackrel{\mathrm{Ph}}{=}$

$(\mathrm{MeO})_{3} \stackrel{\oplus}{\mathrm{P}}-\mathrm{J}_{\ominus}^{\mathrm{Ph}}$

dipole $=5.62$

dipole $=4.62$

x: 0.22

y: -1.55

z: $\mathbf{5 . 4 0}$

x: 0.10

y: $-\mathbf{2 . 1 2}$

z: 4.10

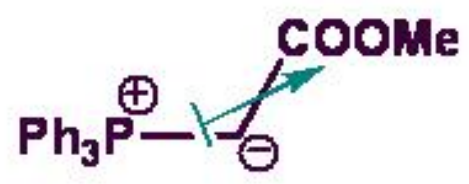

dipole $=5.32 \mathrm{D}$

x: 0.05

$y:-2.38$

z: 4.75

dipole $=\mathbf{2 . 1 2}$

x: 0.35

y: -1.62

z: $\mathbf{2 . 1 2}$ 


\section{Energies and Geometries}

\begin{abstract}
A. Model reaction: $\quad \begin{array}{lllll}0 & 1.21832 & 2.99857 & -0.38722\end{array}$ $\mathrm{Me}_{3} \mathrm{PCH}_{2}+\mathrm{CH}_{2} \mathrm{O}$

\section{$\mathrm{CH}_{2} \mathrm{O}$ (B3LYP 6-31G*)}

$\mathrm{E}\left(\mathrm{B} 3 \mathrm{LYP} 6-31 \mathrm{G}^{*}\right)=-114.50053$

$\mathrm{E}\left(\mathrm{B} 3 \mathrm{LYP} 6-311+\mathrm{G}^{* *}\right)=-114.54171$

$\mathrm{E}(\mathrm{B} 3 \mathrm{LYP} 6-31 \mathrm{G} *(\mathrm{THF}))=-114.50646$

$\mathrm{E}\left(\mathrm{B} 3 \mathrm{LYP} 6-311+\mathrm{G}^{* *}(\mathrm{THF})\right)=$

$-114.54912$

H $0.92813 \quad 1.18417 \quad-1.25682$

\section{$\mathrm{CH}_{2} \mathrm{O}(\mathrm{MP2}$ 6-311+G**)}

$\mathrm{E}\left(\mathrm{MP} 26-311+\mathrm{G}^{* *}\right)=-114.24161$

$\begin{array}{llll}\text { C } & 1.22341 & 1.77961 & -0.37371\end{array}$

H $1.52367 \quad 1.20627 \quad 0.52195$

O $1.21835 \quad 2.99235-0.38713$

H $0.92830 \quad 1.18441 \quad-1.25631$
\end{abstract}

$\mathrm{E}\left(\mathrm{MP} 26-311+\mathrm{G}^{* *}\right)=-114.24151$

$\mathrm{E}(\mathrm{QCISD}(\mathrm{T})$ 6-31G*) $=-114.24151$

$\mathrm{E}(\mathrm{G} 3(\mathrm{MP} 2))=-114.35802$

C $1.22600 \quad 1.80351 \quad-.36579$

H $1.52638 \quad 1.22092 \quad .53039$

O $1.22090 \quad 3.00997 \quad-.37915$

H $0.93075 \quad 1.19868$ - 1.24865

\section{$\mathrm{CH}_{2} \mathrm{O}($ B3LYP 6-31+G*)}

$\mathrm{E}\left(\mathrm{B} 3 \mathrm{LYP} 6-31+\mathrm{G}^{*}\right)=-114.50887$

C $1.22603 \quad 1.80147 \quad-.36570$

H $1.52753 \quad 1.22820 \quad .53382$

O $1.22088 \quad 3.01059 \quad-.37921$

H $0.92958 \quad 1.20587 \quad-1.25211$

\section{$\mathrm{CH}_{2} \mathrm{O}$ (B3LYP 6-311+G**)}

$\mathrm{E}\left(\mathrm{B} 3 \mathrm{LYP} 6-311+\mathrm{G}^{* *}\right)=-114.54176$

$\begin{array}{llll}\text { C } & 1.22600 & 1.80562 & -.36579\end{array}$

H $1.52692 \quad 1.22945 \quad .53201$

O $1.22090 \quad 3.00732 \quad-.37914$

H $\quad 93019 \quad 1.20705 \quad-1.25031$

\section{$\mathrm{CH}_{2} \mathrm{O}$ (MP2 6-31G*)}

$\mathrm{E}(\mathrm{MP} 2$ 6-31G*) $=-114.16774$

$\mathrm{E}(\mathrm{QCISD}(\mathrm{T})$ 6-31G*) $=-114.19110$

C $1.22343 \quad 1.77941 \quad-0.37367$

H $1.52268 \quad 1.20224 \quad 0.51898$

O $1.21833 \quad 3.00037 \quad-0.38719$

H $0.92930 \quad 1.18062-1.25331$

\section{$\mathrm{CH}_{2} \mathrm{O}$ (MP2 6-31+G*)}

$\mathrm{E}\left(\mathrm{MP} 26-31+\mathrm{G}^{*}\right)=-114.17707$

$\begin{array}{llll}\text { C } & 1.22345 & 1.77397 & -0.37362\end{array}$

H $1.52384 \quad 1.20593 \quad 0.52246$

\section{$\mathrm{CH}_{2} \mathrm{O}$ (B3LYP 6-31G*(THF))}

$\mathrm{E}\left(\mathrm{B} 3 \mathrm{LYP} 6-31 \mathrm{G}^{*}(\mathrm{THF})\right)=$ $-114.50644$

$\begin{array}{cccr}\text { C } & 1.22611 & 1.80002 & -.36557 \\ \text { H } & 1.52632 & 1.22175 & .53026 \\ \text { O } & 1.22083 & 3.01248 & -.37930 \\ \text { H } & .93072 & 1.19961 & -1.24866\end{array}$

\section{$\mathrm{Me}_{3} \mathrm{PCH}_{2}$ (B3LYP 6-31G*)}

$\mathrm{E}\left(\mathrm{B} 3 \mathrm{LYP} 6-31 \mathrm{G}^{*}\right)=-500.35268$

$\mathrm{E}\left(\mathrm{B} 3 \mathrm{LYP} 6-311+\mathrm{G}^{* *}\right)=-500.43674$

$\mathrm{E}\left(\mathrm{B} 3 \mathrm{LYP} 6-31 \mathrm{G}^{*}(\mathrm{THF})\right)=$

$-500.36147$

$\mathrm{E}(\mathrm{B} 3 \mathrm{LYP} 6-311+\mathrm{G} * *(\mathrm{THF}))=$ $-500.44803$

$\mathrm{E}\left(\mathrm{MP} 26-311+\mathrm{G}^{* *}\right)==-499.36122$

$\mathrm{E}(\mathrm{QCISD}(\mathrm{T})$ 6-31G*) $=-499.26334$

$\mathrm{E}(\mathrm{G} 3(\mathrm{MP} 2))=-499.61770$
$\begin{array}{llll}\text { C } & 1.63932 & -.11183 & .44414\end{array}$
$\begin{array}{llll}\text { P } & 1.90966 & .46629 & -1.11083\end{array}$
$\begin{array}{llll}\text { C } & 2.65093 & -.54705 & -2.50580\end{array}$
$\begin{array}{llll}\text { C } & .33923 & 1.03285 & -1.87617\end{array}$
C $3.04136 \quad 1.91253 \quad-1.07891$
H $\quad .82210 \quad-.81633 \quad .58021$
H $\quad 2.02205 \quad-1.42709 \quad-2.67877$
H $3.64325 \quad-.89742 \quad-2.20174$
$\begin{array}{llll}\text { H } & 2.74388 & .01378 & -3.44448\end{array}$
H $\quad .49780 \quad 1.37718 \quad-2.90315$
H $\quad-.08547 \quad 1.84040 \quad-1.27516$
H $\quad-.37453 \quad .20274 \quad-1.89340$
H $3.25334 \quad 2.27583 \quad-2.08954$
H $3.98606 \quad 1.62148 \quad-.60805$
$\begin{array}{llll}\mathrm{H} & 2.59120 & 2.71074 & -.48397\end{array}$
H $\quad 2.50885 \quad-.26543 \quad 1.07871$

\section{$\mathrm{Me}_{3} \mathrm{PCH}_{2}(\mathrm{~B} 3 \mathrm{~L}$ YP 6-31+G*)}

$\mathrm{E}\left(\mathrm{B} 3 \mathrm{LYP} 6-31+\mathrm{G}^{*}\right)=-500.36400$

$\begin{array}{llll}\text { C } & 1.64948 & -.13044 & .43832 \\ \text { P } & 1.91126 & .47152 & -1.11541 \\ \text { C } & 2.65190 & -.54934 & -2.50030 \\ \text { C } & .33394 & 1.03276 & -1.86956 \\ \text { C } & 3.04484 & 1.91529 & -1.07029 \\ \text { H } & .81886 & -.81607 & .58910 \\ \text { H } & 2.02043 & -1.42908 & -2.66781 \\ \text { H } & 3.64208 & -.90113 & -2.18956 \\ \text { H } & 2.74825 & .00746 & -3.44181 \\ \text { H } & .48086 & 1.36652 & -2.90225 \\ \text { H } & -.08497 & 1.84666 & -1.27184 \\ \text { H } & -.37952 & .20141 & -1.86843 \\ \text { H } & 3.27253 & 2.27642 & -2.07883 \\ \text { H } & 3.98133 & 1.62059 & -.58418 \\ \text { H } & 2.58917 & 2.71663 & -.48258 \\ \text { H } & 2.51114 & -.26316 & 1.08841\end{array}$

$\mathrm{Me}_{3} \mathrm{PCH}_{2}$ (B3LYP 6-311+G**)

$\mathrm{E}\left(\mathrm{B} 3 \mathrm{LYP} 6-311+\mathrm{G}^{* *}\right)=-500.43691$

$\begin{array}{llll}\text { C } & 1.65057 & -.12757 & .42608\end{array}$

$\begin{array}{llll}\text { P } & 1.90957 & .47494 & -1.12094\end{array}$

$\begin{array}{llll}\text { C } 2.64932 & -.55048 & -2.49586\end{array}$

$\begin{array}{llll}\text { C } & 33269 & 1.03189 & -1.86587\end{array}$

$\begin{array}{llll}\text { C } 3.04117 & 1.91342 & -1.06803\end{array}$

$\begin{array}{llll}\mathrm{H} & .82221 & -.81137 & .57250\end{array}$

H $2.01643-1.42688-2.65344$

$\begin{array}{llll}\text { H } & 3.63380 & -.89966 & -2.17594\end{array}$

H $2.74886-.00200 \quad-3.43778$

H $\quad .47123 \quad 1.36406 \quad-2.89674$

H $-.080691 .84176 \quad-1.26468$

$\begin{array}{llll}\mathrm{H} & -.37398 & .19922 & -1.85538\end{array}$

$\begin{array}{llll}\text { H } & 3.28108 & 2.27461 & -2.07003\end{array}$

$\begin{array}{llll}\mathrm{H} & 3.96587 & 1.61164 & -.57102\end{array}$

H $2.58056 \quad 2.70965 \quad-.48320$

H $2.51142 \quad-.26167 \quad 1.07136$

\section{$\mathrm{Me}_{3} \mathrm{PCH}_{2}(\mathrm{MP} 2$ 6-31G*)}

$\mathrm{E}\left(\mathrm{MP} 26-31 \mathrm{G}^{*}\right)=-499.17532$

$\mathrm{E}(\mathrm{QCISD}(\mathrm{T})$ 6-31G*) $=-499.26332$
$\begin{array}{llll}\text { C } & 1.61298 & -0.07627 & 0.46656\end{array}$
$\begin{array}{llll}\text { P } & 1.89095 & 0.48209 & -1.09046\end{array}$
$\begin{array}{llll}\text { C } & 2.62489 & -0.52597 & -2.46641\end{array}$
$\begin{array}{llll}\text { C } & 0.33319 & 1.04052 & -1.85260\end{array}$
C $3.01752 \quad 1.91356-1.06026$
H $0.79969 \quad-0.78449 \quad 0.59571$
$\begin{array}{llll}\text { H } & 1.99603 & -1.40349 & -2.64310\end{array}$
H $3.61657-0.87610-2.16522$
$\begin{array}{llll}\mathrm{H} & 2.71728 & 0.03755 & -3.40159\end{array}$
$\begin{array}{llll}\mathrm{H} & 0.49866 & 1.41684 & -2.86549\end{array}$
H $-0.10896 \quad 1.82257 \quad-1.23348$ 


\begin{abstract}
H $-0.36632 \quad 0.20156 \quad-1.90281$
$\begin{array}{llll}\text { H } & 3.20185 & 2.29552 & -2.06776\end{array}$

H $3.97302 \quad 1.61305 \quad-0.62159$

$\begin{array}{llll}\mathrm{H} & 2.58374 & 2.69866 & -0.43907\end{array}$

$\begin{array}{llll}\text { H } & 2.48569 & -0.23535 & 1.09331\end{array}$
\end{abstract}

\section{$\mathrm{Me}_{3} \mathrm{PCH}_{2}$ (MP2 6-31+G*)}

$\begin{array}{lrrr}\mathrm{E}(\mathrm{MP} 2 & \left.6-31+\mathrm{G}^{*}\right)=-499.19171 \\ \mathrm{C} & -1.60284 & 0.02233 & -0.87847 \\ \mathrm{P} & -0.11893 & 0.00174 & -0.09034 \\ \mathrm{C} & 1.50583 & -0.02132 & -0.96879 \\ \mathrm{C} & 0.05309 & 1.46687 & 0.96837 \\ \mathrm{C} & 0.01099 & -1.46743 & 0.96876 \\ \mathrm{H} & -1.88678 & 0.95020 & -1.36696 \\ \mathrm{H} & 1.57057 & 0.86270 & -1.60972 \\ \mathrm{H} & 1.54604 & -0.90770 & -1.60848 \\ \mathrm{H} & 2.35788 & -0.03260 & -0.28000 \\ \mathrm{H} & 1.03862 & 1.50467 & 1.43960 \\ \mathrm{H} & -0.72789 & 1.45189 & 1.73035 \\ \mathrm{H} & -0.08072 & 2.36035 & 0.35234 \\ \mathrm{H} & 0.99596 & -1.53525 & 1.43775 \\ \mathrm{H} & -0.15165 & -2.35652 & 0.35332 \\ \mathrm{H} & -0.76736 & -1.42837 & 1.73257 \\ \mathrm{H} & -1.91308 & -0.89822 & -1.36480\end{array}$

$\mathrm{Me}_{3} \mathrm{PCH}_{2}(\mathrm{MP2}$ 6-311+G**)

$\begin{array}{lrrr}\mathrm{E}(\mathrm{MP} 2 & \left.6-311+\mathrm{G}^{* *}\right)=-499.36200 \\ \mathrm{C} & 1.62486 & -0.09110 & 0.44685 \\ \mathrm{P} & 1.89201 & 0.49289 & -1.10593 \\ \mathrm{C} & 2.62200 & -0.51896 & -2.46803 \\ \mathrm{C} & 0.32987 & 1.04366 & -1.84988 \\ \mathrm{C} & 3.01715 & 1.91725 & -1.05787 \\ \mathrm{H} & 0.80832 & -0.79652 & 0.57264 \\ \mathrm{H} & 1.98885 & -1.39582 & -2.63158 \\ \mathrm{H} & 3.61023 & -0.86788 & -2.15475 \\ \mathrm{H} & 2.71560 & 0.04024 & -3.40562 \\ \mathrm{H} & 0.47303 & 1.36678 & -2.88422 \\ \mathrm{H} & -0.08228 & 1.85994 & -1.25440 \\ \mathrm{H} & -0.37790 & 0.21031 & -1.83266 \\ \mathrm{H} & 3.25789 & 2.26957 & -2.06418 \\ \mathrm{H} & 3.94082 & 1.61532 & -0.55651 \\ \mathrm{H} & 2.55560 & 2.71898 & -0.47909 \\ \mathrm{H} & 2.50073 & -0.24442 & 1.07097\end{array}$

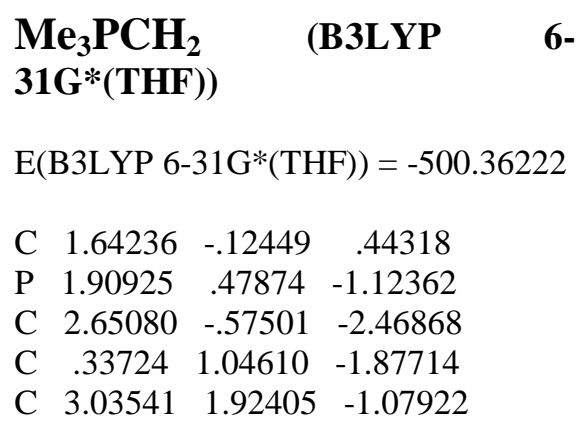

\begin{abstract}
Н $\quad .88099 \quad-.90486 \quad .50541$
$\begin{array}{llll}\text { H } & 2.01308 & -1.45303 & -2.62212\end{array}$

$\begin{array}{llll}\mathrm{H} & 3.63690 & -.92403 & -2.14165\end{array}$

$\begin{array}{llll}\mathrm{H} & 2.75798 & -.04045 & -3.42058\end{array}$

$\mathrm{H} \quad .48905 \quad 1.36116 \quad-2.91487$

$\begin{array}{llll}\mathrm{H} & -.06991 & 1.88087 & -1.29929\end{array}$

$\begin{array}{llll}\mathrm{H} & -.38980 & .22624 & -1.86407\end{array}$

$\begin{array}{llll}\mathrm{H} & 3.26031 & 2.27691 & -2.09107\end{array}$

$\begin{array}{llll}\text { H } & 3.97499 & 1.64061 & -.59183\end{array}$

$\begin{array}{llll}\mathrm{H} & 2.57583 & 2.73298 & -.50432\end{array}$

H $2.55166 \quad-.35436 \quad 1.00272$
\end{abstract}

\section{VDW complex (B3LYP 6-31G*)}

$\mathrm{E}\left(\mathrm{B} 3 \mathrm{LYP} 6-31 \mathrm{G}^{*}\right)=-614.86812$

E(B3LYP 6-311+G**) $=-614.98888$

$\mathrm{E}\left(\mathrm{B} 3 \mathrm{LYP} 6-31 \mathrm{G}^{*}(\mathrm{THF})\right)=$

$-614.88000$

$\mathrm{E}\left(\mathrm{B} 3 \mathrm{LYP} 6-311+\mathrm{G}^{* *}(\mathrm{THF})\right)=$

$-615.00354$

$\mathrm{E}\left(\mathrm{MP} 26-311+\mathrm{G}^{* *}\right)=-613.61400$

$\mathrm{E}(\mathrm{QCISD}(\mathrm{T})$ 6-31G*) $=-613.46804$

$\mathrm{E}(\mathrm{G} 3(\mathrm{MP} 2))=-613.98356$

$\begin{array}{lccc}\mathrm{C} & -.02497 & -.14664 & .84365 \\ \mathrm{O} & -.06597 & .36033 & 1.97544 \\ \mathrm{P} & 3.10092 & .21244 & 1.29434 \\ \mathrm{C} & 2.14257 & -.41551 & .00780 \\ \mathrm{H} & -.14631 & -1.23480 & .69336 \\ \mathrm{C} & 2.61170 & 1.94229 & 1.60984 \\ \mathrm{C} & 2.74395 & -.72917 & 2.81657 \\ \mathrm{C} & 4.95207 & .25415 & 1.18511 \\ \mathrm{H} & -.22656 & .45036 & -.06421 \\ \mathrm{H} & 2.26915 & -1.48507 & -.16495 \\ \mathrm{H} & 3.20399 & 2.37830 & 2.42032 \\ \mathrm{H} & 1.54908 & 1.92753 & 1.87627 \\ \mathrm{H} & 2.75595 & 2.53860 & .70309 \\ \mathrm{H} & 3.34076 & -.36151 & 3.65703 \\ \mathrm{H} & 2.96871 & -1.78869 & 2.65693 \\ \mathrm{H} & 1.67471 & -.60602 & 3.02179 \\ \mathrm{H} & 5.39956 & .68395 & 2.08933 \\ \mathrm{H} & 5.25323 & .85242 & .31917 \\ \mathrm{H} & 5.33200 & -.76343 & 1.04841 \\ \mathrm{H} & 2.18475 & .17281 & -.90978\end{array}$

VDW complex (B3LYP 6-
$\left.31+\mathrm{G}^{*}\right)$

6- $\quad \mathrm{E}\left(\mathrm{B} 3 \mathrm{LYP} 6-31+\mathrm{G}^{*}\right)=-614.88420$

$\begin{array}{llll}\text { C } & -.17113 & -.13983 & .86554\end{array}$

$\begin{array}{llll}\mathrm{O} & -.21580 & .35392 & 1.99406\end{array}$

$\begin{array}{llll}\text { P } & 3.13265 & .20605 & 1.28442\end{array}$

$\begin{array}{llll}\text { C } & 2.20698 & -.42966 & -.01449\end{array}$

$\begin{array}{llll}\mathrm{H} & -.22428 & -1.22965 & .70646\end{array}$

$\begin{array}{llll}\text { C } & 2.64349 & 1.93996 & 1.59440\end{array}$

$\begin{array}{llll}\text { C } & 2.78049 & -.74308 & 2.80625\end{array}$

$\begin{array}{llll}\text { C } & 4.98918 & .27152 & 1.21599\end{array}$

$\begin{array}{cccc}\mathrm{H} & -.29320 & .47463 & -.04189 \\ \mathrm{H} & 2.31038 & -1.50380 & -.17545 \\ \mathrm{H} & 3.24486 & 2.38049 & 2.39639 \\ \mathrm{H} & 1.58416 & 1.95551 & 1.86947 \\ \mathrm{H} & 2.78786 & 2.52735 & .68104 \\ \mathrm{H} & 3.38630 & -.38195 & 3.64377 \\ \mathrm{H} & 3.00831 & -1.80093 & 2.63551 \\ \mathrm{H} & 1.71659 & -.63706 & 3.04086 \\ \mathrm{H} & 5.41751 & .70680 & 2.12790 \\ \mathrm{H} & 5.29850 & .87208 & .35367 \\ \mathrm{H} & 5.38268 & -.74226 & 1.08444 \\ \mathrm{H} & 2.23203 & .15635 & -.93445\end{array}$

VDW complex (B3LYP 6$\left.311+G^{* *}\right)$

$\mathrm{E}\left(\mathrm{B} 3 \mathrm{LYP} 6-311+\mathrm{G}^{* *}\right)=-614.98997$

$\begin{array}{cccc}\mathrm{C} & -.21961 & -.13724 & .88335 \\ \mathrm{O} & -.25621 & .35552 & 2.00164 \\ \mathrm{P} & 3.14010 & .20795 & 1.28575 \\ \mathrm{C} & 2.23989 & -.42615 & -.02022 \\ \mathrm{H} & -.24554 & -1.22718 & .72270 \\ \mathrm{C} & 2.64274 & 1.93646 & 1.58741 \\ \mathrm{C} & 2.77414 & -.74563 & 2.79629 \\ \mathrm{C} & 4.99274 & .27319 & 1.22757 \\ \mathrm{H} & -.32247 & .47248 & -.02884 \\ \mathrm{H} & 2.35005 & -1.49618 & -.18088 \\ \mathrm{H} & 3.25821 & 2.39639 & 2.36279 \\ \mathrm{H} & 1.59303 & 1.94507 & 1.88642 \\ \mathrm{H} & 2.75525 & 2.50397 & .66085 \\ \mathrm{H} & 3.39085 & -.41351 & 3.63353 \\ \mathrm{H} & 2.97334 & -1.80243 & 2.60484 \\ \mathrm{H} & 1.71664 & -.61838 & 3.03462 \\ \mathrm{H} & 5.42012 & .70548 & 2.13718 \\ \mathrm{H} & 5.29942 & .87063 & .36623 \\ \mathrm{H} & 5.37870 & -.73977 & 1.09419 \\ \mathrm{H} & 2.27079 & .16376 & -.93344\end{array}$

VDW complex (MP2 6-31G*)

$\mathrm{E}\left(\mathrm{MP} 26-31 \mathrm{G}^{*}\right)=-613.35842$

$\mathrm{E}(\mathrm{QCISD}(\mathrm{T})$ 6-31G*) $=-613.46863$
$\begin{array}{llll}\text { C } & -0.22001 & -0.11001 & 0.94355\end{array}$
$\begin{array}{llll}\text { O } & -0.21751 & 0.39740 & 2.06754\end{array}$
$\begin{array}{llll}\text { P } & 3.13159 & 0.19065 & 1.24285\end{array}$
$\begin{array}{llll}\text { C } & 2.23288 & -0.43881 & -0.05362\end{array}$
$\begin{array}{llll}\text { H } & -0.24693 & -1.19888 & 0.79260\end{array}$
$\begin{array}{llll}\text { C } & 2.63063 & 1.90810 & 1.55392\end{array}$
$\begin{array}{llll}\text { C } & 2.76199 & -0.74818 & 2.75261\end{array}$
$\begin{array}{llll}\text { C } & 4.97577 & 0.28261 & 1.24459\end{array}$
$\begin{array}{llll}\mathrm{H} & -0.33044 & 0.49147 & 0.02968\end{array}$
$\begin{array}{llll}\mathrm{H} & 2.35509 & -1.50892 & -0.21565\end{array}$
$\begin{array}{llll}\mathrm{H} & 3.21907 & 2.34890 & 2.36287\end{array}$
$\begin{array}{llll}\mathrm{H} & 1.57152 & 1.91198 & 1.82041\end{array}$
$\begin{array}{llll}\mathrm{H} & 2.77836 & 2.50210 & 0.64782\end{array}$
$\begin{array}{llll}\mathrm{H} & 3.35430 & -0.38478 & 3.59645\end{array}$ 


\begin{abstract}
H $2.99130 \quad-1.80512 \quad 2.59149$
$\begin{array}{llll}\mathrm{H} & 1.69765 & -0.63813 & 2.97125\end{array}$

$\begin{array}{llll}\mathrm{H} & 5.36260 & 0.71997 & 2.17139\end{array}$

$\begin{array}{llll}\mathrm{H} & 5.30948 & 0.89055 & 0.39890\end{array}$

H $5.38926-0.72304 \quad 1.12707$

$\begin{array}{llll}\text { H } & 2.27273 & 0.15448 & -0.96615\end{array}$
\end{abstract}

\section{VDW complex (MP2 6-31+G*)}

\begin{tabular}{lrrr}
\multicolumn{4}{l}{ E(MP2 6-31+G*) $=-613.38175$} \\
C & -0.27203 & -0.11307 & 0.94256 \\
O & -0.26194 & 0.39563 & 2.06875 \\
P & 3.14015 & 0.19294 & 1.24695 \\
C & 2.25978 & -0.44247 & -0.06472 \\
H & -0.28959 & -1.20199 & 0.79991 \\
C & 2.63891 & 1.91114 & 1.54963 \\
C & 2.77059 & -0.75269 & 2.75170 \\
C & 4.98198 & 0.28756 & 1.25488 \\
H & -0.37349 & 0.49552 & 0.03388 \\
H & 2.37605 & -1.51493 & -0.22526 \\
H & 3.23728 & 2.35762 & 2.34947 \\
H & 1.58159 & 1.93047 & 1.82602 \\
H & 2.78167 & 2.49629 & 0.63563 \\
H & 3.37354 & -0.39902 & 3.59342 \\
H & 2.99455 & -1.81012 & 2.57892 \\
H & 1.70892 & -0.64526 & 2.98834 \\
H & 5.36604 & 0.72574 & 2.18382 \\
H & 5.31595 & 0.89490 & 0.40764 \\
H & 5.39572 & -0.71884 & 1.13585 \\
H & 2.29360 & 0.15290 & -0.97787
\end{tabular}

\section{VDW complex (MP2 6-311+G**)}

$E\left(M P 26-311+G^{* *}\right)=-613.61696$

$\begin{array}{llll}\text { C } & -0.30115 & -0.10935 & 0.95398\end{array}$

$\begin{array}{llll}\text { O } & -0.29371 & 0.39400 & 2.06859\end{array}$

$\begin{array}{llll}\text { P } & 3.14493 & 0.19444 & 1.24975\end{array}$

$\begin{array}{llll}\text { C } & 2.27128 & -0.43990 & -0.06028\end{array}$

$\begin{array}{llll}\text { H } & -0.30500 & -1.19996 & 0.80473\end{array}$

$\begin{array}{llll}\text { C } & 2.64375 & 1.91025 & 1.54510\end{array}$

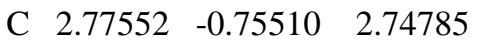

$\begin{array}{llll}\text { C } & 4.98156 & 0.29024 & 1.26085\end{array}$

$\begin{array}{llll}\mathrm{H} & -0.38885 & 0.49654 & 0.03915\end{array}$

H $2.40035-1.51070 \quad-0.21650$

$\begin{array}{llll}\mathrm{H} & 3.24698 & 2.36448 & 2.33545\end{array}$

$\begin{array}{llll}\mathrm{H} & 1.58850 & 1.92768 & 1.82541\end{array}$

$\begin{array}{llll}\mathrm{H} & 2.77973 & 2.47983 & 0.62149\end{array}$

$\begin{array}{llll}\mathrm{H} & 3.38430 & -0.41323 & 3.58891\end{array}$

$\begin{array}{llll}\mathrm{H} & 2.99180 & -1.81003 & 2.55731\end{array}$

H $1.71560 \quad-0.64332 \quad 2.98559$

$\begin{array}{llll}\text { H } & 5.36491 & 0.72803 & 2.18903\end{array}$

$\begin{array}{llll}\mathrm{H} & 5.31060 & 0.89682 & 0.41233\end{array}$

H $\quad 5.39038 \quad-0.71702 \quad 1.14059$

$\begin{array}{llll}\text { H } & 2.31781 & 0.15865 & -0.96980\end{array}$

TS1 (B3L YP 6-31G*)
$\mathrm{E}(\mathrm{B} 3 \mathrm{LYP}$ 6-31G*) $=-614.86732$

E(B3LYP 6-311+G**) $=-614.98804$

$\mathrm{E}\left(\mathrm{B} 3 \mathrm{LYP} \quad 6-31 \mathrm{G}^{*}(\mathrm{THF})\right)=$ -

614.88439

$\mathrm{E}\left(\mathrm{B} 3 \mathrm{LYP} 6-311+\mathrm{G}^{* *}(\mathrm{THF})\right)=$ $-615.00863$

$\mathrm{E}\left(\mathrm{MP} 26-311+\mathrm{G}^{* *}\right)=-613.61286$

$\mathrm{E}(\mathrm{QCISD}(\mathrm{T})$ 6-31G*) $=-613.46571$

$\mathrm{E}(\mathrm{G} 3(\mathrm{MP} 2))=-613.97971$

$\begin{array}{lccc}\mathrm{C} & -.17160 & -.16053 & .79444 \\ \mathrm{O} & -.29908 & .33757 & 1.95153 \\ \mathrm{P} & 2.73773 & .27662 & 1.46879 \\ \mathrm{C} & 1.76549 & -.36145 & .16027 \\ \mathrm{H} & -.39012 & -1.23664 & .63161 \\ \mathrm{C} & 2.23306 & 1.99791 & 1.77659 \\ \mathrm{C} & 2.36760 & -.67319 & 2.97643 \\ \mathrm{C} & 4.57479 & .29158 & 1.29435 \\ \mathrm{H} & -.45466 & .43857 & -.09631 \\ \mathrm{H} & 1.96476 & -1.42197 & -.01485 \\ \mathrm{H} & 2.78051 & 2.42569 & 2.62218 \\ \mathrm{H} & 1.15640 & 1.94348 & 1.98795 \\ \mathrm{H} & 2.41265 & 2.60667 & .88475 \\ \mathrm{H} & 2.91063 & -.27445 & 3.83886 \\ \mathrm{H} & 2.63826 & -1.72417 & 2.83341 \\ \mathrm{H} & 1.28173 & -.57622 & 3.11228 \\ \mathrm{H} & 5.04832 & .71718 & 2.18707 \\ \mathrm{H} & 4.85916 & .88869 & .42234 \\ \mathrm{H} & 4.94135 & -.72948 & 1.15010 \\ \mathrm{H} & 1.88799 & .21787 & -.75879\end{array}$

\section{TS1 (B3LYP 6-31+G*)}

$\mathrm{E}\left(\mathrm{B} 3 \mathrm{LYP} 6-31+\mathrm{G}^{*}\right)=-614.88341$

$$
\begin{array}{lccc}
\mathrm{C} & -.22474 & -.27819 & .82230 \\
\mathrm{O} & -.38479 & .32848 & 1.91615 \\
\mathrm{P} & 2.75418 & .20249 & 1.49233 \\
\mathrm{C} & 1.79706 & -.36416 & .14908 \\
\mathrm{H} & -.30684 & -1.38185 & .78852 \\
\mathrm{C} & 2.26927 & 1.91953 & 1.85721 \\
\mathrm{C} & 2.37173 & -.80725 & 2.96175 \\
\mathrm{C} & 4.59756 & .21030 & 1.36412 \\
\mathrm{H} & -.53518 & .19839 & -.12625 \\
\mathrm{H} & 1.99130 & -1.40793 & -.11114 \\
\mathrm{H} & 2.80611 & 2.30319 & 2.73081 \\
\mathrm{H} & 1.18779 & 1.90751 & 2.04179 \\
\mathrm{H} & 2.48878 & 2.55536 & .99268 \\
\mathrm{H} & 2.94634 & -.46963 & 3.83034 \\
\mathrm{H} & 2.60980 & -1.85749 & 2.76154 \\
\mathrm{H} & 1.29676 & -.70063 & 3.14906 \\
\mathrm{H} & 5.05448 & .59523 & 2.28449 \\
\mathrm{H} & 4.90550 & .83941 & .52257 \\
\mathrm{H} & 4.96004 & -.80736 & 1.18491 \\
\mathrm{H} & 1.87069 & .29194 & -.72185
\end{array}
$$

TS1 (B3LYP 6-311+G**)
$\mathrm{E}\left(\mathrm{B} 3 \mathrm{LYP} 6-311+\mathrm{G}^{* *}\right)=-614.98814$

$$
\begin{array}{cccc}
\mathrm{C} & -.21229 & -.23217 & .79893 \\
\mathrm{O} & -. .35817 & .25237 & 1.94937 \\
\mathrm{P} & 2.74843 & .20972 & 1.49743 \\
\mathrm{C} & 1.80491 & -.38735 & .16416 \\
\mathrm{H} & -.36924 & -1.31269 & .63035 \\
\mathrm{C} & 2.24459 & 1.92071 & 1.84269 \\
\mathrm{C} & 2.37652 & -.79295 & 2.96639 \\
\mathrm{C} & 4.58307 & .23319 & 1.34273 \\
\mathrm{H} & -.46564 & .37083 & -.09127 \\
\mathrm{H} & 1.99537 & -1.43986 & -.04607 \\
\mathrm{H} & 2.80565 & 2.33474 & 2.68241 \\
\mathrm{H} & 1.17408 & 1.88868 & 2.06751 \\
\mathrm{H} & 2.41627 & 2.53465 & .95579 \\
\mathrm{H} & 2.94399 & -.44818 & 3.83266 \\
\mathrm{H} & 2.62558 & -1.83702 & 2.76397 \\
\mathrm{H} & 1.30082 & -.69798 & 3.14280 \\
\mathrm{H} & 5.05088 & .62518 & 2.25045 \\
\mathrm{H} & 4.86722 & .85774 & .49352 \\
\mathrm{H} & 4.94443 & -.78100 & 1.16173 \\
\mathrm{H} & 1.90815 & .22837 & -.72942
\end{array}
$$

\section{TS1 (MP2 6-31G*)}

$\mathrm{E}\left(\mathrm{MP} 26-31 \mathrm{G}^{*}\right)=-613.35686$

$\mathrm{E}\left(\mathrm{QCISD}(\mathrm{T}) 6-31 \mathrm{G}^{*}\right)=-613.46576$

$$
\begin{array}{rrrc}
\mathrm{C} & -0.20191 & -0.17837 & 0.83842 \\
\mathrm{O} & -0.33217 & 0.30179 & 1.99868 \\
\mathrm{P} & 2.74886 & 0.22529 & 1.46230 \\
\mathrm{C} & 1.81135 & -0.37478 & 0.14099 \\
\mathrm{H} & -0.36360 & -1.25631 & 0.66110 \\
\mathrm{C} & 2.23854 & 1.92696 & 1.79912 \\
\mathrm{C} & 2.36821 & -0.76229 & 2.92835 \\
\mathrm{C} & 4.57798 & 0.26890 & 1.35608 \\
\mathrm{H} & -0.44418 & 0.43453 & -0.04768 \\
\mathrm{H} & 1.98977 & -1.43299 & -0.05808 \\
\mathrm{H} & 2.78240 & 2.34017 & 2.65240 \\
\mathrm{H} & 1.16442 & 1.88400 & 2.00864 \\
\mathrm{H} & 2.42406 & 2.54939 & 0.91966 \\
\mathrm{H} & 2.91424 & -0.39762 & 3.80213 \\
\mathrm{H} & 2.63437 & -1.80745 & 2.74921 \\
\mathrm{H} & 1.28757 & -0.67115 & 3.08133 \\
\mathrm{H} & 5.01272 & 0.67571 & 2.27511 \\
\mathrm{H} & 4.88329 & 0.89228 & 0.51162 \\
\mathrm{H} & 4.96210 & -0.74220 & 1.19784 \\
\mathrm{H} & 1.90964 & 0.23506 & -0.75884
\end{array}
$$

\section{TS1 (MP2 6-31+G*)}

$\mathrm{E}\left(\mathrm{MP} 26-31+\mathrm{G}^{*}\right)=-613.38033$
$\begin{array}{llll}\text { C } & -0.25689 & -0.18600 & 0.82651\end{array}$
$\begin{array}{llll}\text { O } & -0.38540 & 0.29414 & 1.98694\end{array}$
$\begin{array}{llll}\text { P } & 2.75209 & 0.22398 & 1.45885\end{array}$
C $1.82735 \quad-0.37802 \quad 0.13150$ 


$\begin{array}{cccc}\mathrm{H} & -0.39166 & -1.26566 & 0.65569 \\ \mathrm{C} & 2.24950 & 1.92854 & 1.79588 \\ \mathrm{C} & 2.37936 & -0.76479 & 2.92686 \\ \mathrm{C} & 4.58204 & 0.27561 & 1.37157 \\ \mathrm{H} & -0.47265 & 0.43516 & -0.05700 \\ \mathrm{H} & 1.99204 & -1.43976 & -0.06608 \\ \mathrm{H} & 2.81658 & 2.34397 & 2.63421 \\ \mathrm{H} & 1.17989 & 1.91446 & 2.03009 \\ \mathrm{H} & 2.42379 & 2.54474 & 0.90833 \\ \mathrm{H} & 2.94918 & -0.41036 & 3.79095 \\ \mathrm{H} & 2.63414 & -1.81229 & 2.73804 \\ \mathrm{H} & 1.30471 & -0.67584 & 3.11752 \\ \mathrm{H} & 5.00822 & 0.68396 & 2.29539 \\ \mathrm{H} & 4.89244 & 0.89966 & 0.52820 \\ \mathrm{H} & 4.97126 & -0.73474 & 1.21429 \\ \mathrm{H} & 1.91166 & 0.23418 & -0.76936\end{array}$

\section{TS1 (MP2 6-311+G**)}

$\mathrm{E}\left(\mathrm{MP} 26-311+\mathrm{G}^{* *}\right)=-613.61363$

C $-0.18932-0.19110 \quad 0.80582$

$\begin{array}{llll}\text { O } & -0.33918 & 0.28554 & 1.96249\end{array}$

$\begin{array}{llll}\mathrm{P} & 2.73894 & 0.23323 & 1.48262\end{array}$

$\begin{array}{llll}\text { C } & 1.79869 & -0.36777 & 0.15990\end{array}$

$\begin{array}{llll}\text { H } & -0.35535 & -1.26916 & 0.62874\end{array}$

$\begin{array}{llll}\text { C } & 2.24512 & 1.93608 & 1.81152\end{array}$

$\begin{array}{llll}\text { C } & 2.37532 & -0.75936 & 2.94350\end{array}$

$\begin{array}{llll}\text { C } & 4.55813 & 0.26563 & 1.35032\end{array}$

$\begin{array}{llll}\mathrm{H} & -0.43498 & 0.42355 & -0.07906\end{array}$

$\begin{array}{llll}\text { H } & 1.98669 & -1.42593 & -0.03554\end{array}$

$\begin{array}{llll}\mathrm{H} & 2.80675 & 2.34875 & 2.65343\end{array}$

$\begin{array}{llll}\mathrm{H} & 1.17267 & 1.91620 & 2.03140\end{array}$

H $2.43196 \quad 2.54079 \quad 0.91984$

H $2.93874 \quad-0.40024 \quad 3.80845$

$\begin{array}{llll}\mathrm{H} & 2.64270 & -1.80061 & 2.74354\end{array}$

$\begin{array}{llll}\mathrm{H} & 1.29774 & -0.67760 & 3.11968\end{array}$

$\begin{array}{llll}\text { H } & 5.00563 & 0.67094 & 2.26412\end{array}$

$\begin{array}{llll}\mathrm{H} & 4.85074 & 0.88628 & 0.49953\end{array}$

H $4.92980 \quad-0.74904 \quad 1.18615$

$\begin{array}{llll}\text { H } & 1.90684 & 0.24475 & -0.73807\end{array}$

\section{OP1 (B3LYP 6-31G*)}

$\mathrm{E}\left(\mathrm{B} 3 \mathrm{LYP} 6-31 \mathrm{G}^{*}\right)=-614.90256$

E(B3LYP 6-311+G**) $=-615.01534$

$\mathrm{E}\left(\mathrm{B} 3 \mathrm{LYP} 6-31 \mathrm{G}^{*}(\mathrm{THF})\right)=-614.91084$

$\mathrm{E}\left(\mathrm{B} 3 \mathrm{LYP} 6-311+\mathrm{G}^{* *}(\mathrm{THF})\right)=$

$-615.02606$

$\mathrm{E}\left(\mathrm{MP} 26-311+\mathrm{G}^{* *}\right)=-613.64918$

$\mathrm{E}(\mathrm{QCISD}(\mathrm{T})$ 6-31G*) $=-613.50884$

$\mathrm{E}(\mathrm{G} 3(\mathrm{MP} 2))=-614.01942$

$\begin{array}{cccc}\mathrm{O} & -.16342 & -.14978 & -.31217 \\ \mathrm{C} & .27664 & .18549 & .98848 \\ \mathrm{C} & 1.74098 & .51222 & .67426 \\ \mathrm{P} & 1.45908 & .14096 & -1.14523 \\ \mathrm{H} & -.27228 & 1.04425 & 1.40735\end{array}$

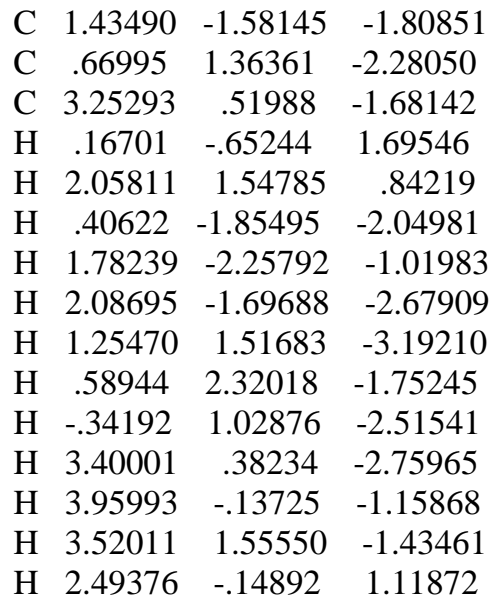

\section{OP1 (B3L YP 6-31+G*)}

$\mathrm{E}\left(\mathrm{B} 3 \mathrm{LYP} 6-31+\mathrm{G}^{*}\right)=-614.91627$

$\begin{array}{cccc}\mathrm{O} & -.17843 & -.15150 & -.30257 \\ \mathrm{C} & .27340 & .18733 & .99743 \\ \mathrm{C} & 1.73633 & .50900 & .67015 \\ \mathrm{P} & 1.46861 & .14232 & -1.15140 \\ \mathrm{H} & -.27458 & 1.04826 & 1.41117 \\ \mathrm{C} & 1.43349 & -1.58022 & -1.81038 \\ \mathrm{C} & .66913 & 1.36079 & -2.28212 \\ \mathrm{C} & 3.26116 & .52255 & -1.68210 \\ \mathrm{H} & .16318 & -.65202 & 1.70148 \\ \mathrm{H} & 2.05614 & 1.54472 & .83673 \\ \mathrm{H} & .40270 & -1.85006 & -2.04885 \\ \mathrm{H} & 1.78106 & -2.25912 & -1.02314 \\ \mathrm{H} & 2.08313 & -1.69522 & -2.68370 \\ \mathrm{H} & 1.25325 & 1.51380 & -3.19498 \\ \mathrm{H} & .58457 & 2.31829 & -1.75539 \\ \mathrm{H} & -.34206 & 1.02094 & -2.51502 \\ \mathrm{H} & 3.40744 & .38613 & -2.76110 \\ \mathrm{H} & 3.96823 & -.13584 & -1.16009 \\ \mathrm{H} & 3.52730 & 1.55870 & -1.43429 \\ \mathrm{H} & 2.48911 & -.15431 & 1.11299\end{array}$

\section{OP1 (B3LYP 6-311+G**)}

$\mathrm{E}\left(\right.$ B3LYP $\left.6-311+\mathrm{G}^{* *}\right)=-615.01614$

$\begin{array}{cccc}\mathrm{O} & -.19273 & -.15455 & -.29968 \\ \mathrm{C} & .26360 & .18429 & .99443 \\ \mathrm{C} & 1.72633 & .50514 & .66528 \\ \mathrm{P} & 1.47698 & .14411 & -1.15404 \\ \mathrm{H} & -.27625 & 1.04542 & 1.41250 \\ \mathrm{C} & 1.43746 & -1.57381 & -1.80603 \\ \mathrm{C} & .67527 & 1.35861 & -2.27641 \\ \mathrm{C} & 3.26471 & .52340 & -1.68089 \\ \mathrm{H} & .15960 & -.64995 & 1.70198 \\ \mathrm{H} & 2.04602 & 1.53779 & .82984 \\ \mathrm{H} & .40561 & -1.84849 & -2.01665 \\ \mathrm{H} & 1.80971 & -2.24400 & -1.02685 \\ \mathrm{H} & 2.06778 & -1.68279 & -2.69038\end{array}$

$\begin{array}{cccc}\mathrm{H} & 1.24736 & 1.49574 & -3.19566 \\ \mathrm{H} & .61489 & 2.31771 & -1.75558 \\ \mathrm{H} & -.34067 & 1.02897 & -2.48566 \\ \mathrm{H} & 3.40622 & .38465 & -2.75634 \\ \mathrm{H} & 3.96876 & -.13302 & -1.15993 \\ \mathrm{H} & 3.52868 & 1.55697 & -1.43587 \\ \mathrm{H} & 2.47716 & -.15736 & 1.10464\end{array}$

OP1 (MP2 6-31G*)

$\mathrm{E}\left(\mathrm{MP} 26-31 \mathrm{G}^{*}\right)=-613.40197$

$\mathrm{E}(\mathrm{QCISD}(\mathrm{T})$ 6-31G*) $=-613.50884$

$\begin{array}{llll}\text { O } & -0.20180 & 0.04228 & -0.34406\end{array}$

$\begin{array}{llll}\text { C } & 0.32102 & 0.03827 & 0.97841\end{array}$

$\begin{array}{llll}\text { C } & 1.73749 & 0.50172 & 0.65364\end{array}$

$\begin{array}{llll}\mathrm{P} & 1.44616 & 0.18251 & -1.15668\end{array}$

$\begin{array}{llll}\text { H } & -0.24089 & 0.71356 & 1.63582\end{array}$

$\begin{array}{llll}\text { C } & 1.38067 & -1.56246 & -1.70398\end{array}$

$\begin{array}{llll}\text { C } & 0.70786 & 1.35588 & -2.35567\end{array}$

$\begin{array}{llll}\text { C } & 3.23262 & 0.50342 & -1.67923\end{array}$

$\begin{array}{llll}\mathrm{H} & 0.31179 & -0.96419 & 1.43447\end{array}$

$\begin{array}{lllll}\mathrm{H} & 1.89480 & 1.57434 & 0.81534\end{array}$

$\begin{array}{llll}\mathrm{H} & 0.36147 & -1.79688 & -2.01235\end{array}$

H $1.62106-2.19696 \quad-0.84484$

$\begin{array}{llll}\mathrm{H} & 2.09028 & -1.77536 & -2.50623\end{array}$

$\begin{array}{llll}\mathrm{H} & 1.27344 & 1.39147 & -3.28935\end{array}$

$\begin{array}{lllll}\mathrm{H} & 0.71648 & 2.35254 & -1.90302\end{array}$

$\begin{array}{llll}\mathrm{H} & -0.33117 & 1.07897 & -2.53401\end{array}$

$\begin{array}{llll}\text { H } & 3.38623 & 0.34291 & -2.75166\end{array}$

$\begin{array}{llll}\text { H } & 3.91604 & -0.16083 & -1.13892\end{array}$

H $3.52265 \quad 1.53555-1.45106$

H $\quad 2.58374 \quad-0.04220 \quad 1.08656$

\section{OP1 (MP2 6-31+G*)}

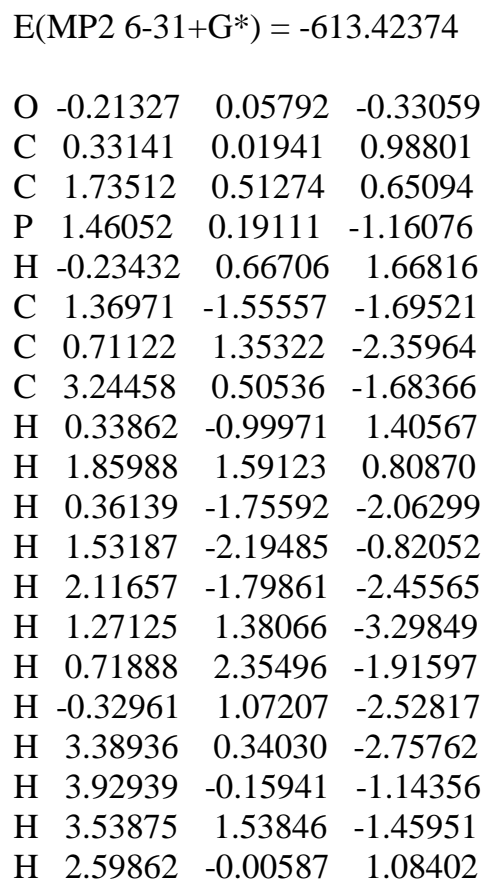




\section{OP1 (MP2 6-311+G**)}

$\mathrm{E}\left(\mathrm{MP} 26-311+\mathrm{G}^{* *}\right)=-613.65117$

$\begin{array}{rrrr}\text { O } & -0.21391 & 0.08491 & -0.32782 \\ \text { C } & 0.34020 & -0.00876 & 0.97086 \\ \text { C } & 1.73645 & 0.52216 & 0.64500 \\ \text { P } & 1.46386 & 0.20495 & -1.16171 \\ \text { H } & -0.22080 & 0.59281 & 1.69431 \\ \text { C } & 1.35897 & -1.54229 & -1.66926 \\ \text { C } & 0.71871 & 1.35800 & -2.36261 \\ \text { C } & 3.24135 & 0.50279 & -1.69268 \\ \text { H } & 0.38326 & -1.04627 & 1.33853 \\ \text { H } & 1.81525 & 1.60415 & 0.79729 \\ \text { H } & 0.36795 & -1.72339 & -2.08794 \\ \text { H } & 1.44942 & -2.16249 & -0.77214 \\ \text { H } & 2.14224 & -1.82006 & -2.37765 \\ \text { H } & 1.27860 & 1.37607 & -3.30058 \\ \text { H } & 0.73648 & 2.35950 & -1.92090 \\ \text { H } & -0.32387 & 1.08302 & -2.52265 \\ \text { H } & 3.37990 & 0.32484 & -2.76444 \\ \text { H } & 3.91950 & -0.16191 & -1.14625 \\ \text { H } & 3.54040 & 1.53572 & -1.47899 \\ \text { H } & 2.61598 & 0.03080 & 1.07279\end{array}$

OP1 (B3LYP 6-31G*(THF))

$\mathrm{E}(\mathrm{B} 3 \mathrm{LYP} 6-31 \mathrm{G} *(\mathrm{THF}))=-614.91184$

$\begin{array}{llll}\text { O } & -.19397 & -.15698 & -.29121\end{array}$

$\begin{array}{llll}\text { C } & .27885 & .19028 & .99631\end{array}$

$\begin{array}{llll}\text { C } & 1.73786 & .50717 & .66012\end{array}$

$\begin{array}{llll}\mathrm{P} & 1.47437 & .14269 & -1.15624\end{array}$

$\begin{array}{llll}\mathrm{H} & -.25443 & 1.05649 & 1.42237\end{array}$

C $1.43633-1.58014 \quad-1.81176$

$\begin{array}{llll}\text { C } & .66966 & 1.36190 & -2.28021\end{array}$

$\begin{array}{llll}\text { C } & 3.26111 & .52485 & -1.68064\end{array}$

H $\quad .18020 \quad-.63690 \quad 1.71872$

$\begin{array}{llll}\mathrm{H} & 2.06029 & 1.54187 & .82357\end{array}$

H $\quad .41293 \quad-1.84564 \quad-2.08560$

H $1.76219 \quad-2.26270-1.01859$

H $2.10531-1.69674-2.66959$

H $1.26015 \quad 1.52195 \quad-3.18732$

H $\quad .57761 \quad 2.31688 \quad-1.75030$

$\mathrm{H} \quad-.33581 \quad 1.01853 \quad-2.53203$

$\begin{array}{llll}\text { H } & 3.41149 & .38502 & -2.75790\end{array}$

H $3.96200 \quad-.13141 \quad-1.14885$

H $3.51707 \quad 1.56248 \quad-1.43085$

$\begin{array}{llll}\mathrm{H} & 2.48996 & -.15842 & 1.09886\end{array}$

\section{TSOP (B3LYP 6-31G*)}

$\mathrm{E}(\mathrm{B} 3 \mathrm{LYP}$ 6-31G*) $=-614.89930$

$\mathrm{E}\left(\mathrm{B} 3 \mathrm{LYP} 6-311+\mathrm{G}^{* *}\right)=-615.00989$

$\mathrm{E}\left(\mathrm{B} 3 \mathrm{LYP} 6-31 \mathrm{G}^{*}(\mathrm{THF})\right)=$

$-614.90555$

$\mathrm{E}\left(\mathrm{B} 3 \mathrm{LYP} 6-311+\mathrm{G}^{* *}(\mathrm{THF})\right)=$
$-615.01816$

$\mathrm{E}\left(\mathrm{MP} 26-311+\mathrm{G}^{* *}\right)=-613.64488$

$\mathrm{E}(\mathrm{QCISD}(\mathrm{T})$ 6-31G*) $=-613.50513$

$\mathrm{E}(\mathrm{G} 3(\mathrm{MP} 2))=-614.01498$

$\begin{array}{cccc}\mathrm{O} & .12767 & .41650 & -.49305 \\ \mathrm{C} & .47859 & .09157 & .86476 \\ \mathrm{C} & 1.94310 & .48967 & .81175 \\ \mathrm{P} & 1.73011 & .71468 & -1.11969 \\ \mathrm{H} & -.14202 & .67008 & 1.55950 \\ \mathrm{H} & .31681 & -.97715 & 1.06004 \\ \mathrm{H} & 2.16036 & 1.43282 & 1.32518 \\ \mathrm{H} & 2.67500 & -.26032 & 1.13176 \\ \mathrm{C} & 2.52949 & -.83395 & -1.73103 \\ \mathrm{H} & 2.78082 & -.74933 & -2.79241 \\ \mathrm{H} & 3.43752 & -1.04909 & -1.16051 \\ \mathrm{H} & 1.83165 & -1.66840 & -1.60674 \\ \mathrm{C} & 3.09330 & 2.00634 & -1.25905 \\ \mathrm{H} & 3.43959 & 2.14332 & -2.28889 \\ \mathrm{H} & 2.70621 & 2.96913 & -.90236 \\ \mathrm{H} & 3.93745 & 1.72864 & -.61841 \\ \mathrm{C} & .86333 & 1.28782 & -2.69785 \\ \mathrm{H} & 1.58100 & 1.38807 & -3.51912 \\ \mathrm{H} & .07951 & .58008 & -2.97974 \\ \mathrm{H} & .39014 & 2.26166 & -2.53117\end{array}$

TSOP (B3LYP 6-31+G*)

$\mathrm{E}\left(\right.$ B3LYP $\left.6-31+\mathrm{G}^{*}\right)=-614.91079$

$\begin{array}{cccc}\mathrm{O} & .15868 & .71074 & -.42896 \\ \mathrm{C} & .49108 & .38864 & .94585 \\ \mathrm{C} & 1.97721 & .14540 & .76275 \\ \mathrm{P} & 1.72918 & .64295 & -1.14429 \\ \mathrm{H} & .23807 & 1.23867 & 1.59088 \\ \mathrm{H} & -.09177 & -.48336 & 1.26428 \\ \mathrm{H} & 2.63592 & .80391 & 1.34019 \\ \mathrm{H} & 2.29007 & -.89258 & .92266 \\ \mathrm{C} & 2.67388 & -.81560 & -1.82351 \\ \mathrm{H} & 2.92758 & -.68868 & -2.88081 \\ \mathrm{H} & 3.58323 & -.97980 & -1.23590 \\ \mathrm{H} & 2.04434 & -1.70921 & -1.72724 \\ \mathrm{C} & 2.88145 & 2.09931 & -1.13467 \\ \mathrm{H} & 3.12266 & 2.43452 & -2.14823 \\ \mathrm{H} & 2.38777 & 2.92495 & -.60772 \\ \mathrm{H} & 3.80150 & 1.85296 & -.59539 \\ \mathrm{C} & .86265 & 1.06152 & -2.77756 \\ \mathrm{H} & 1.59564 & 1.17350 & -3.58535 \\ \mathrm{H} & .15820 & .26790 & -3.05084 \\ \mathrm{H} & .29334 & 1.99235 & -2.68331\end{array}$

\section{TSOP (B3LYP 6-311+G**)}

$\mathrm{E}\left(\mathrm{B} 3 \mathrm{LYP} 6-311+\mathrm{G}^{* *}\right)=-615.00967$

$\begin{array}{cccc}\mathrm{O} & .15716 & .68738 & -.44034 \\ \mathrm{C} & .47917 & .35989 & .93583 \\ \mathrm{C} & 1.97399 & .17396 & .77280\end{array}$

$\begin{array}{cccc}\mathrm{P} & 1.73161 & .65049 & -1.14380 \\ \mathrm{H} & .18551 & 1.18849 & 1.58636 \\ \mathrm{H} & -.07118 & -.53647 & 1.23426 \\ \mathrm{H} & 2.59440 & .86473 & 1.34792 \\ \mathrm{H} & 2.32743 & -.84492 & .94522 \\ \mathrm{C} & 2.65651 & -.81790 & -1.81078 \\ \mathrm{H} & 2.91416 & -.69548 & -2.86401 \\ \mathrm{H} & 3.55715 & -.99336 & -1.21982 \\ \mathrm{H} & 2.01136 & -1.69634 & -1.71541 \\ \mathrm{C} & 2.89724 & 2.09292 & -1.14007 \\ \mathrm{H} & 3.14867 & 2.41512 & -2.15166 \\ \mathrm{H} & 2.40600 & 2.92419 & -.62616 \\ \mathrm{H} & 3.80535 & 1.84176 & -.58959 \\ \mathrm{C} & .87612 & 1.07978 & -2.77470 \\ \mathrm{H} & 1.61095 & 1.18415 & -3.57698 \\ \mathrm{H} & .16534 & .29644 & -3.04628 \\ \mathrm{H} & .31970 & 2.01400 & -2.67665\end{array}$

\section{TSOP (MP2 6-31G*)}

$\mathrm{E}\left(\mathrm{MP} 26-31 \mathrm{G}^{*}\right)=-613.39849$

$\mathrm{E}(\mathrm{QCISD}(\mathrm{T})$ 6-31G*) $=-613.50507$

$\begin{array}{rrrr}\mathrm{O} & 0.16389 & 0.68317 & -0.46302 \\ \mathrm{C} & 0.49389 & 0.34474 & 0.91603 \\ \mathrm{C} & 1.99204 & 0.23778 & 0.75765 \\ \mathrm{P} & 1.74465 & 0.68034 & -1.13978 \\ \mathrm{H} & 0.14962 & 1.14750 & 1.57548 \\ \mathrm{H} & -0.01451 & -0.58503 & 1.18968 \\ \mathrm{H} & 2.56370 & 0.97096 & 1.33428 \\ \mathrm{H} & 2.40064 & -0.75803 & 0.95324 \\ \mathrm{C} & 2.63677 & -0.79713 & -1.78974 \\ \mathrm{H} & 2.87309 & -0.70150 & -2.85199 \\ \mathrm{H} & 3.55204 & -0.96574 & -1.21679 \\ \mathrm{H} & 1.98874 & -1.67054 & -1.65754 \\ \mathrm{C} & 2.92124 & 2.10068 & -1.14943 \\ \mathrm{H} & 3.17999 & 2.41481 & -2.16321 \\ \mathrm{H} & 2.44122 & 2.94291 & -0.63904 \\ \mathrm{H} & 3.82711 & 1.84193 & -0.59543 \\ \mathrm{C} & 0.89516 & 1.12124 & -2.75580 \\ \mathrm{H} & 1.62617 & 1.22873 & -3.56325 \\ \mathrm{H} & 0.17828 & 0.34453 & -3.03423 \\ \mathrm{H} & 0.34589 & 2.06077 & -2.65413\end{array}$

TSOP (MP2 6-31+G*)

$\mathrm{E}\left(\mathrm{MP} 26-31+\mathrm{G}^{*}\right)=-613.41715$
$\begin{array}{lllll}\mathrm{O} & 0.14551 & 0.69435 & -0.46207\end{array}$
$\begin{array}{llll}\text { C } & 0.47853 & 0.35086 & 0.91855\end{array}$
$\begin{array}{llll}\text { C } & 1.97633 & 0.24390 & 0.75251\end{array}$
$\begin{array}{llll}\mathrm{P} & 1.73377 & 0.69538 & -1.14715\end{array}$
$\begin{array}{llll}\mathrm{H} & 0.13495 & 1.15462 & 1.57709\end{array}$
$\begin{array}{llll}\mathrm{H} & -0.03222 & -0.57936 & 1.18604\end{array}$
$\begin{array}{llll}\mathrm{H} & 2.55230 & 0.97509 & 1.32977\end{array}$
$\begin{array}{llll}\mathrm{H} & 2.38575 & -0.75450 & 0.94007\end{array}$
$\begin{array}{llll}\text { C } & 2.63545 & -0.77386 & -1.80197\end{array}$
$\begin{array}{llll}\text { H } & 2.87417 & -0.67042 & -2.86412\end{array}$ 


$\begin{array}{rrrr}\mathrm{H} & 3.55222 & -0.93881 & -1.22812 \\ \mathrm{H} & 1.99344 & -1.65399 & -1.67543 \\ \mathrm{C} & 2.91318 & 2.11294 & -1.15120 \\ \mathrm{H} & 3.17269 & 2.42943 & -2.16528 \\ \mathrm{H} & 2.43663 & 2.95608 & -0.63658 \\ \mathrm{H} & 3.82029 & 1.84902 & -0.59941 \\ \mathrm{C} & 0.88331 & 1.14067 & -2.75870 \\ \mathrm{H} & 1.62034 & 1.25057 & -3.56179 \\ \mathrm{H} & 0.16602 & 0.36450 & -3.04252 \\ \mathrm{H} & 0.33164 & 2.08011 & -2.65570\end{array}$

\section{TSOP (MP2 6-311+G**)}

$\mathrm{E}\left(\mathrm{MP} 26-311+\mathrm{G}^{* *}\right)=-613.64508$

$\begin{array}{lrrr}\mathrm{O} & 0.15785 & 0.69286 & -0.46984 \\ \mathrm{C} & 0.48293 & 0.35249 & 0.90375 \\ \mathrm{C} & 1.98487 & 0.24381 & 0.74974 \\ \mathrm{P} & 1.73067 & 0.69611 & -1.14918 \\ \mathrm{H} & 0.14449 & 1.15574 & 1.56400 \\ \mathrm{H} & -0.02123 & -0.57962 & 1.17323 \\ \mathrm{H} & 2.55726 & 0.97804 & 1.32303 \\ \mathrm{H} & 2.39174 & -0.75446 & 0.93227 \\ \mathrm{C} & 2.62483 & -0.77449 & -1.79562 \\ \mathrm{H} & 2.83923 & -0.69306 & -2.86379 \\ \mathrm{H} & 3.54941 & -0.92388 & -1.23200 \\ \mathrm{H} & 1.98589 & -1.64989 & -1.63338 \\ \mathrm{C} & 2.90046 & 2.11450 & -1.14403 \\ \mathrm{H} & 3.13959 & 2.45868 & -2.15295 \\ \mathrm{H} & 2.42366 & 2.93658 & -0.59837 \\ \mathrm{H} & 3.81334 & 1.84049 & -0.60885 \\ \mathrm{C} & 0.89725 & 1.14021 & -2.76484 \\ \mathrm{H} & 1.64300 & 1.24795 & -3.55863 \\ \mathrm{H} & 0.18251 & 0.36473 & -3.05374 \\ \mathrm{H} & 0.34658 & 2.07980 & -2.66682\end{array}$

\section{TSOP (B3LYP 6-31G*(THF))}

$\mathrm{E}\left(\mathrm{B} 3 \mathrm{LYP} 6-31 \mathrm{G}^{*}(\mathrm{THF})\right)=$ $-614.90578$
$\begin{array}{llll}\text { O } & .12677 & .52208 & -.46955\end{array}$
$\begin{array}{llll}\text { C } & .47034 & .17901 & .89582\end{array}$
$\begin{array}{llll}\text { C } & 1.97088 & .37197 & .79927\end{array}$
$\begin{array}{llll}\text { P } & 1.73942 & .69241 & -1.12901\end{array}$
$\begin{array}{llll}\mathrm{H} & -.04673 & .85459 & 1.58816\end{array}$
$\begin{array}{llll}\mathrm{H} & .15712 & -.84946 & 1.11604\end{array}$
$\begin{array}{llll}\mathrm{H} & 2.34200 & 1.24777 & 1.34241\end{array}$
$\begin{array}{llll}\mathrm{H} & 2.58582 & -.49480 & 1.06388\end{array}$
$\begin{array}{llll}\text { C } & 2.55879 & -.84082 & -1.75947\end{array}$
$\begin{array}{llll}\mathrm{H} & 2.78627 & -.76013 & -2.82680\end{array}$
H $3.47804-1.03755-1.19964$
H $1.87948-1.68912 \quad-1.61492$
C $3.01648 \quad 2.05524 \quad-1.21511$
$\begin{array}{llll}\mathrm{H} & 3.32620 & 2.27093 & -2.24289\end{array}$
$\begin{array}{llll}\mathrm{H} & 2.58133 & 2.96806 & -.78874\end{array}$
$\begin{array}{llll}\mathrm{H} & 3.88810 & 1.78408 & -.60992\end{array}$
$\begin{array}{llll}\text { C } & .87745 & 1.21091 & -2.73664\end{array}$

$\begin{array}{cccc}\mathrm{H} & 1.61096 & 1.30819 & -3.54491 \\ \mathrm{H} & .12245 & .47418 & -3.02936 \\ \mathrm{H} & .37629 & 2.17702 & -2.60629\end{array}$

\section{OP2 (B3LYP 6-31G*)}

$\mathrm{E}\left(\mathrm{B} 3 \mathrm{LYP} 6-31 \mathrm{G}^{*}\right)=-614.89969$

$\mathrm{E}\left(\mathrm{B} 3 \mathrm{LYP} 6-311+\mathrm{G}^{* *}\right)=-615.00926$

$\mathrm{E}\left(\mathrm{B} 3 \mathrm{LYP} \quad 6-31 \mathrm{G}^{*}(\mathrm{THF})\right)=-$

614.90590

$\mathrm{E}\left(\mathrm{B} 3 \mathrm{LYP} 6-311+\mathrm{G}^{* *}(\mathrm{THF})\right)=$

$-615.01758$

$\mathrm{E}\left(\mathrm{MP} 26-311+\mathrm{G}^{* *}\right)=-613.64426$

$\mathrm{E}(\mathrm{QCISD}(\mathrm{T})$ ) 6-31G*) $=-613.50502$

$\mathrm{E}(\mathrm{G} 3(\mathrm{MP} 2))=-614.01456$

$\begin{array}{lclc}\mathrm{O} & .14057 & .58929 & -.49362 \\ \mathrm{C} & .41979 & .32300 & .90363 \\ \mathrm{C} & 1.93382 & .28429 & .82793 \\ \mathrm{P} & 1.74039 & .64282 & -1.11468 \\ \mathrm{H} & .00617 & 1.13034 & 1.51907 \\ \mathrm{H} & -.05403 & -.62100 & 1.19729 \\ \mathrm{H} & 2.44266 & 1.06897 & 1.39777 \\ \mathrm{H} & 2.38381 & -.68076 & 1.08363 \\ \mathrm{C} & 2.73796 & -.82364 & -1.67544 \\ \mathrm{H} & 3.00655 & -.76024 & -2.73393 \\ \mathrm{H} & 3.64060 & -.92122 & -1.06462 \\ \mathrm{H} & 2.13238 & -1.72585 & -1.52852 \\ \mathrm{C} & 2.85465 & 2.13219 & -1.13993 \\ \mathrm{H} & 3.13455 & 2.42198 & -2.15698 \\ \mathrm{H} & 2.31617 & 2.96981 & -.68071 \\ \mathrm{H} & 3.75202 & 1.94152 & -.54358 \\ \mathrm{C} & .93672 & .98045 & -2.79988 \\ \mathrm{H} & 1.70025 & 1.09310 & -3.57813 \\ \mathrm{H} & .27067 & .15705 & -3.07739 \\ \mathrm{H} & .33618 & 1.89507 & -2.76189\end{array}$

\section{OP2 (B3L YP 6-31+G*)}

$\mathrm{E}\left(\mathrm{B} 3 \mathrm{LYP} 6-31+\mathrm{G}^{*}\right)=-614.91076$
O $\quad .13675$
$\begin{array}{ll}.58845 & -.48993\end{array}$
$\begin{array}{llll}\text { C } & .41685 & .32199 & .90915\end{array}$
$\begin{array}{llll}\text { C } & 1.93161 & .28458 & .82892\end{array}$
$\begin{array}{llll}\mathrm{P} & 1.74191 & .64325 & -1.11769\end{array}$
$\begin{array}{llll}\mathrm{H} & .00170 & 1.13090 & 1.52172\end{array}$
$\begin{array}{llll}\mathrm{H} & -.05812 & -.62235 & 1.19992\end{array}$
$\begin{array}{llll}\mathrm{H} & 2.44268 & 1.07045 & 1.39667\end{array}$
$\begin{array}{llll}\mathrm{H} & 2.38382 & -.68123 & 1.08145\end{array}$
$\begin{array}{llll}\text { C } & 2.74124 & -.82408 & -1.67664\end{array}$
$\begin{array}{llll}\text { H } & 3.01054 & -.75942 & -2.73556\end{array}$
$\begin{array}{llll}\mathrm{H} & 3.64460 & -.92033 & -1.06549\end{array}$
$\begin{array}{llll}\mathrm{H} & 2.13604 & -1.72760 & -1.53078\end{array}$
$\begin{array}{llll}\text { C } & 2.85771 & 2.13305 & -1.14111\end{array}$
$\begin{array}{llll}\mathrm{H} & 3.13743 & 2.42205 & -2.15909\end{array}$
$\begin{array}{llll}\text { H } & 2.32029 & 2.97204 & -.68153\end{array}$
$\begin{array}{llll}\mathrm{H} & 3.75623 & 1.94082 & -.54568\end{array}$
$\begin{array}{llll}\text { C } & .93576 & .98054 & -2.80039\end{array}$
$\begin{array}{llll}\mathrm{H} & 1.70069 & 1.09214 & -3.57811\end{array}$
$\begin{array}{llll}\mathrm{H} & .26794 & .15748 & -3.07812\end{array}$
$\begin{array}{llll}\mathrm{H} & .33541 & 1.89634 & -2.76230\end{array}$

\section{OP2 (B3LYP 6-311+G**)}

E $\left(\right.$ B3LYP $\left.6-311+\mathrm{G}^{* *}\right)=-615.00968$

$\begin{array}{lllc}\text { O } & .13903 & .58799 & -.48870 \\ \text { C } & .41802 & .32099 & .91034 \\ \text { C } & 1.93063 & .28496 & .82973 \\ \text { P } & 1.74222 & .64377 & -1.11944 \\ \text { H } & .00528 & 1.12672 & 1.52344 \\ \text { H } & -.05289 & -.62192 & 1.20145 \\ \text { H } & 2.43981 & 1.06968 & 1.39344 \\ \text { H } & 2.38238 & -.67771 & 1.07870 \\ \text { C } & 2.73889 & -.82000 & -1.67708 \\ \text { H } & 3.01470 & -.74905 & -2.73030 \\ \text { H } & 3.63285 & -.92312 & -1.05963 \\ \text { H } & 2.12716 & -1.71676 & -1.54175 \\ \text { C } & 2.85593 & 2.12936 & -1.14343 \\ \text { H } & 3.14313 & 2.40831 & -2.15837 \\ \text { H } & 2.31161 & 2.96713 & -.69779 \\ \text { H } & 3.74458 & 1.94222 & -.53822 \\ \text { C } & .93743 & .98028 & -2.79759 \\ \text { H } & 1.70082 & 1.09174 & -3.57176 \\ \text { H } & .27214 & .15868 & -3.07096 \\ \text { H } & .33888 & 1.89260 & -2.75555\end{array}$

\section{OP2 (MP2 6-31G*)}

$\mathrm{E}\left(\mathrm{MP} 26-31 \mathrm{G}^{*}\right)=-613.39849$

$\mathrm{E}(\mathrm{QCISD}(\mathrm{T})$ 6-31G* $)=-613.50502$
O 0.13872
$\begin{array}{ll}0.59141 & -0.50555\end{array}$
$\begin{array}{llll}\text { C } & 0.42640 & 0.32329 & 0.89832\end{array}$
$\begin{array}{llll}\text { C } & 1.93351 & 0.28888 & 0.80529\end{array}$
$\begin{array}{lllll}\mathrm{P} & 1.74407 & 0.64312 & -1.11761\end{array}$
$\begin{array}{llll}\mathrm{H} & 0.01362 & 1.13132 & 1.51026\end{array}$
$\begin{array}{llll}\mathrm{H} & -0.04569 & -0.62067 & 1.18795\end{array}$
$\begin{array}{llll}\mathrm{H} & 2.44165 & 1.07353 & 1.37353\end{array}$
$\begin{array}{llll}\mathrm{H} & 2.38350 & -0.67550 & 1.05875\end{array}$
$\begin{array}{llll}\text { C } & 2.73354 & -0.81200 & -1.66896\end{array}$
$\begin{array}{llll}\text { H } & 3.00872 & -0.74803 & -2.72420\end{array}$
H $3.63148 \quad-0.91054 \quad-1.05395$
H $2.12449-1.71118 \quad-1.52510$
C $2.84911 \quad 2.11952-1.13852$
$\begin{array}{llll}\mathrm{H} & 3.13447 & 2.40736 & -2.15295\end{array}$
$\begin{array}{lllll}\mathrm{H} & 2.30786 & 2.95552 & -0.68207\end{array}$
$\begin{array}{lllll}\mathrm{H} & 3.74258 & 1.92840 & -0.53894\end{array}$
$\begin{array}{llll}\text { C } & 0.94255 & 0.97705 & -2.78258\end{array}$
$\begin{array}{lllll}\mathrm{H} & 1.70033 & 1.08962 & -3.56421\end{array}$
$\begin{array}{lllll}\mathrm{H} & 0.27661 & 0.15510 & -3.05749\end{array}$
$\begin{array}{llll}\mathrm{H} & 0.34438 & 1.89096 & -2.74196\end{array}$

\section{OP2 (MP2 6-31+G*)}

$\mathrm{E}\left(\mathrm{MP} 26-31+\mathrm{G}^{*}\right)=-613.41715$ 


$\begin{array}{rrrr}\mathrm{O} & 0.12901 & 0.59004 & -0.49928 \\ \mathrm{C} & 0.42052 & 0.32077 & 0.90710 \\ \mathrm{C} & 1.92755 & 0.28992 & 0.80683 \\ \mathrm{P} & 1.74266 & 0.64390 & -1.11991 \\ \mathrm{H} & 0.00625 & 1.12990 & 1.51649 \\ \mathrm{H} & -0.05059 & -0.62491 & 1.19254 \\ \mathrm{H} & 2.43758 & 1.07733 & 1.37198 \\ \mathrm{H} & 2.38219 & -0.67452 & 1.05720 \\ \mathrm{C} & 2.73735 & -0.80770 & -1.67056 \\ \mathrm{H} & 3.01319 & -0.74087 & -2.72662 \\ \mathrm{H} & 3.63720 & -0.90208 & -1.05552 \\ \mathrm{H} & 2.13242 & -1.71116 & -1.52713 \\ \mathrm{C} & 2.85391 & 2.11550 & -1.14229 \\ \mathrm{H} & 3.14017 & 2.40012 & -2.15859 \\ \mathrm{H} & 2.31774 & 2.95603 & -0.68533 \\ \mathrm{H} & 3.74863 & 1.91990 & -0.54379 \\ \mathrm{C} & 0.93974 & 0.97761 & -2.78193 \\ \mathrm{H} & 1.70344 & 1.08905 & -3.55947 \\ \mathrm{H} & 0.27227 & 0.15585 & -3.05871 \\ \mathrm{H} & 0.34063 & 1.89251 & -2.74300\end{array}$

\section{OP2 (MP2 6-311+G**)}

$\mathrm{E}\left(\mathrm{MP} 26-311+\mathrm{G}^{* *}\right)=-613.64508$

$\begin{array}{lrrr}\mathrm{O} & 0.14227 & 0.59086 & -0.50447 \\ \mathrm{C} & 0.42668 & 0.32259 & 0.89379 \\ \mathrm{C} & 1.93744 & 0.29012 & 0.80483 \\ \mathrm{P} & 1.73929 & 0.64452 & -1.12180 \\ \mathrm{H} & 0.01802 & 1.13097 & 1.50634 \\ \mathrm{H} & -0.03798 & -0.62468 & 1.18105 \\ \mathrm{H} & 2.44430 & 1.08030 & 1.36527 \\ \mathrm{H} & 2.38965 & -0.67461 & 1.05014 \\ \mathrm{C} & 2.72508 & -0.80890 & -1.66546 \\ \mathrm{H} & 2.97773 & -0.76464 & -2.72744 \\ \mathrm{H} & 3.63254 & -0.88869 & -1.06135 \\ \mathrm{H} & 2.12128 & -1.70587 & -1.48723 \\ \mathrm{C} & 2.84212 & 2.11574 & -1.13724 \\ \mathrm{H} & 3.10755 & 2.42474 & -2.15099 \\ \mathrm{H} & 2.30604 & 2.93834 & -0.65058 \\ \mathrm{H} & 3.74319 & 1.90984 & -0.55369 \\ \mathrm{C} & 0.95206 & 0.97810 & -2.78649 \\ \mathrm{H} & 1.72335 & 1.08737 & -3.55528 \\ \mathrm{H} & 0.28622 & 0.15751 & -3.06740 \\ \mathrm{H} & 0.35502 & 1.89356 & -2.75199\end{array}$

\section{OP2 (B3L YP 6-31G*(THF))}

$\mathrm{E}(\mathrm{B} 3 \mathrm{LYP} 6-31 \mathrm{G} *(\mathrm{THF}))=$
-614.90614
$\begin{array}{lccc} & & \\ \mathrm{O} & .13102 & .58855 & -.48858 \\ \mathrm{C} & .42023 & .32387 & .91226 \\ \mathrm{C} & 1.93249 & .28417 & .82423 \\ \mathrm{P} & 1.74653 & .64235 & -1.11667 \\ \mathrm{H} & .00997 & 1.13263 & 1.52862 \\ \mathrm{H} & -.05467 & -.61815 & 1.21162\end{array}$

$\begin{array}{llll}\mathrm{H} & 2.44592 & 1.06961 & 1.38854 \\ \mathrm{H} & 2.38259 & -.68247 & 1.07313 \\ \mathrm{C} & 2.73748 & -.82521 & -1.67791 \\ \mathrm{H} & 3.01753 & -.75143 & -2.73333 \\ \mathrm{H} & 3.63344 & -.92847 & -1.05777 \\ \mathrm{H} & 2.12726 & -1.72721 & -1.54468 \\ \mathrm{C} & 2.85148 & 2.13531 & -1.14047 \\ \mathrm{H} & 3.13373 & 2.41923 & -2.15894 \\ \mathrm{H} & 2.31112 & 2.97483 & -.68508 \\ \mathrm{H} & 3.74777 & 1.94538 & -.54153 \\ \mathrm{C} & .93739 & .97961 & -2.80163 \\ \mathrm{H} & 1.70687 & 1.09208 & -3.57380 \\ \mathrm{H} & .27571 & .15378 & -3.08677 \\ \mathrm{H} & .33983 & 1.89775 & -2.76882 \#\end{array}$

TS2 (B3LYP 6-31G*)

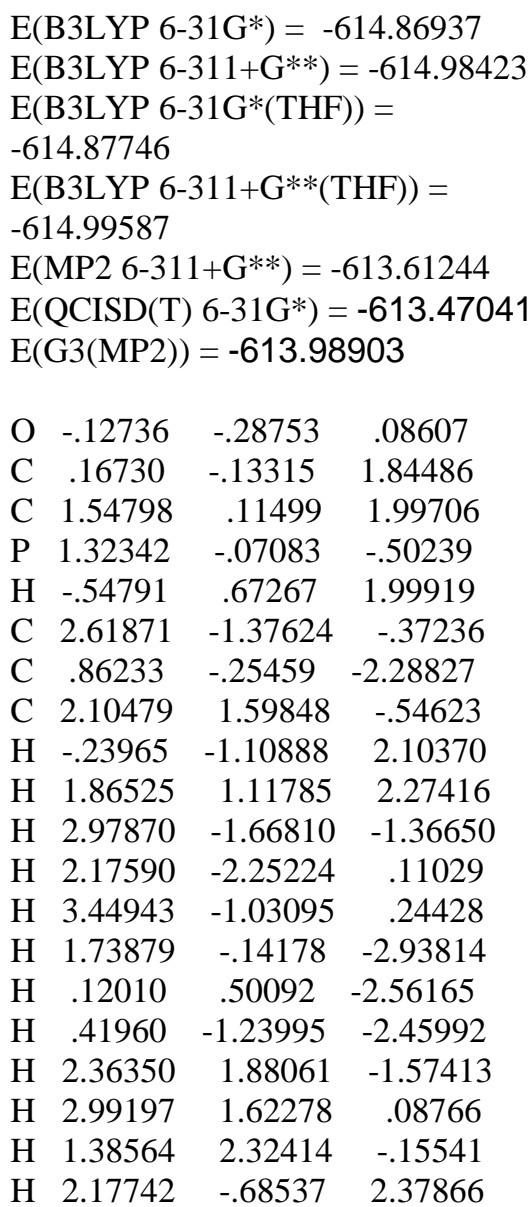

\section{TS2 (B3LYP 6-31+G*)}

$\mathrm{E}\left(\mathrm{B} 3 \mathrm{LYP} 6-31+\mathrm{G}^{*}\right)=-614.88277$

$\begin{array}{lccc}\mathrm{O} & -.11552 & -.28388 & .10146 \\ \mathrm{C} & .15240 & -.13572 & 1.84418 \\ \mathrm{C} & 1.53641 & .11345 & 2.01477 \\ \mathrm{P} & 1.33367 & -.06944 & -.51123 \\ \mathrm{H} & -.56333 & .66991 & 2.00053 \\ \mathrm{C} & 2.62206 & -1.38070 & -.37924\end{array}$

$\begin{array}{cccc}\mathrm{C} & .84968 & -.25718 & -2.28886 \\ \mathrm{C} & 2.10698 & 1.60300 & -.55377 \\ \mathrm{H} & -.25539 & -1.11124 & 2.10459 \\ \mathrm{H} & 1.85126 & 1.11708 & 2.29642 \\ \mathrm{H} & 2.98456 & -1.66849 & -1.37438 \\ \mathrm{H} & 2.17084 & -2.25659 & .09692 \\ \mathrm{H} & 3.45310 & -1.04367 & .24254 \\ \mathrm{H} & 1.72074 & -.14650 & -2.94707 \\ \mathrm{H} & .10468 & .49880 & -2.55565 \\ \mathrm{H} & .40351 & -1.24293 & -2.45309 \\ \mathrm{H} & 2.37182 & 1.88085 & -1.58197 \\ \mathrm{H} & 2.99055 & 1.63573 & .08582 \\ \mathrm{H} & 1.37979 & 2.32569 & -.17108 \\ \mathrm{H} & 2.16328 & -.68834 & 2.40193\end{array}$

TS2 (B3LYP 6-311+G**)

$\mathrm{E}\left(\mathrm{B} 3 \mathrm{LYP} 6-311+\mathrm{G}^{* *}\right)=-614.98465$

$\begin{array}{llll}\text { O } & -.095136 & -.28158 & .11728\end{array}$

$\begin{array}{llll}\text { C } & .138863 & -.13817 & 1.83316\end{array}$

$\begin{array}{llll}\text { C } & 1.520783 & .11415 & 2.03282\end{array}$

$\begin{array}{llll}\mathrm{P} & 1.342330 & -.06877 & -.51839\end{array}$

$\begin{array}{llll}\mathrm{H} & -.576689 & .66363 & 1.99589\end{array}$

C $\quad 2.628204 \quad-1.37311 \quad-.39111$

$\begin{array}{llll}\text { C } & .826344 & -.25934 & -2.27982\end{array}$

$\begin{array}{llll}\text { C } & 2.116184 & 1.59556 & -.56484\end{array}$

$\begin{array}{llll}\mathrm{H} & -.268738 & -1.10913 & 2.10234\end{array}$

$\begin{array}{llll}\mathrm{H} & 1.818933 & 1.11355 & 2.33502\end{array}$

$\mathrm{H} \quad 3.004210 \quad-1.63998 \quad-1.38373$

$\begin{array}{llll}\mathrm{H} & 2.172087 & -2.25468 & .06238\end{array}$

$\begin{array}{llll}\mathrm{H} & 3.442092 & -1.04106 & .24959\end{array}$

$\begin{array}{llll}\mathrm{H} & 1.682480 & -.14834 & -2.95167\end{array}$

$\begin{array}{llll}\mathrm{H} & .077164 & .49414 & -2.52761\end{array}$

$\begin{array}{llll}\mathrm{H} & .379489 & -1.24339 & -2.42878\end{array}$

$\begin{array}{llll}\mathrm{H} & 2.398777 & 1.85935 & -1.58880\end{array}$

$\begin{array}{llll}\mathrm{H} & 2.982717 & 1.62814 & .09183\end{array}$

$\begin{array}{llll}\mathrm{H} & 1.384220 & 2.31966 & -.20315\end{array}$

$\begin{array}{llll}\mathrm{H} & 2.131828 & -.68421 & 2.44290\end{array}$

\section{TS2 (MP2 6-31G*)}

$\mathrm{E}\left(\mathrm{MP} 26-31 \mathrm{G}^{*}\right)=-613.36303$

$\mathrm{E}(\mathrm{QCISD}(\mathrm{T})$ 6-31G*) $=-613.46576$
$\begin{array}{llll}\text { O } & -0.14121 & -0.29010 & 0.07123\end{array}$
$\begin{array}{llll}\text { C } & 0.17901 & -0.13199 & 1.82991\end{array}$
$\begin{array}{llll}\text { C } & 1.55334 & 0.11363 & 1.96957\end{array}$
$\begin{array}{llll}\text { P } & 1.32089 & -0.07094 & -0.50175\end{array}$
$\begin{array}{llll}\mathrm{H} & -0.53267 & 0.67603 & 1.98696\end{array}$
$\begin{array}{llll}\text { C } & 2.60577 & -1.36596 & -0.36210\end{array}$
$\begin{array}{llll}\text { C } & 0.87084 & -0.25231 & -2.27381\end{array}$
$\begin{array}{llll}\text { C } & 2.09560 & 1.58621 & -0.53470\end{array}$
$\begin{array}{llll}\mathrm{H} & -0.22437 & -1.10823 & 2.09123\end{array}$
$\begin{array}{llll}\mathrm{H} & 1.87670 & 1.11612 & 2.23171\end{array}$
$\begin{array}{lllll}\mathrm{H} & 2.96363 & -1.66736 & -1.35198\end{array}$
$\begin{array}{llll}\mathrm{H} & 2.16115 & -2.23482 & 0.12968\end{array}$
H $3.43805 \quad-1.01835 \quad 0.24942$ 


$\begin{array}{rrrr}\mathrm{H} & 1.74826 & -0.13840 & -2.91900 \\ \mathrm{H} & 0.13021 & 0.50199 & -2.54752 \\ \mathrm{H} & 0.43069 & -1.23675 & -2.44587 \\ \mathrm{H} & 2.35143 & 1.87582 & -1.55912 \\ \mathrm{H} & 2.98402 & 1.60863 & 0.09593 \\ \mathrm{H} & 1.37640 & 2.30606 & -0.13592 \\ \mathrm{H} & 2.18818 & -0.68648 & 2.33705\end{array}$

\section{TS2 (MP2 6-31+G*)}

$\mathrm{E}\left(\mathrm{MP} 26-31+\mathrm{G}^{*}\right)=-613.38536$

$\begin{array}{rrrr}\text { O } & -0.14046 & -0.29685 & -0.03861 \\ \text { C } & 0.18298 & -0.14019 & 1.69352 \\ \text { C } & 1.56471 & 0.10683 & 1.82864 \\ \text { P } & 1.30753 & -0.08300 & -0.66965 \\ \text { H } & -0.52567 & 0.66898 & 1.86331 \\ \text { C } & 2.58682 & -1.38308 & -0.54948 \\ \text { C } & 0.80156 & -0.27238 & -2.42324 \\ \text { C } & 2.07508 & 1.57513 & -0.72149 \\ \text { H } & -0.21680 & -1.11548 & 1.96702 \\ \text { H } & 1.88963 & 1.11096 & 2.09020 \\ \text { H } & 2.93340 & -1.67786 & -1.54681 \\ \text { H } & 2.14242 & -2.25362 & -0.05813 \\ \text { H } & 3.42900 & -1.04464 & 0.05557 \\ \text { H } & 1.66446 & -0.16212 & -3.09013 \\ \text { H } & 0.05394 & 0.48256 & -2.67997 \\ \text { H } & 0.35449 & -1.25763 & -2.57877 \\ \text { H } & 2.32254 & 1.85457 & -1.75220 \\ \text { H } & 2.96986 & 1.60872 & -0.09849 \\ \text { H } & 1.35522 & 2.29608 & -0.32297 \\ \text { H } & 2.20211 & -0.69413 & 2.19530\end{array}$

TS2 (MP2 6-311+G**)

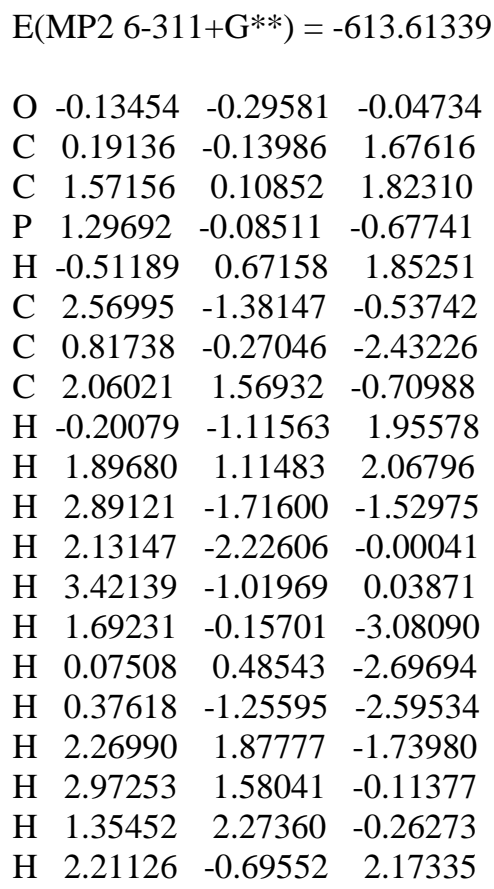

\section{TS2 (B3LYP 6-31G*(THF))}

$\mathrm{E}\left(\mathrm{B} 3 \mathrm{LYP} \quad 6-31 \mathrm{G}^{*}(\mathrm{THF})\right)=$ 614.87769

$\begin{array}{cccc}\mathrm{O} & -.12236 & -.28455 & .09997 \\ \mathrm{C} & .15340 & -.13827 & 1.83320 \\ \mathrm{C} & 1.53665 & .10973 & 1.99318 \\ \mathrm{P} & 1.33824 & -.06772 & -.50379 \\ \mathrm{H} & -.56035 & .66644 & 2.00671 \\ \mathrm{C} & 2.61656 & -1.38526 & -.37288 \\ \mathrm{C} & .85482 & -.25407 & -2.28287 \\ \mathrm{C} & 2.10157 & 1.60692 & -.54756 \\ \mathrm{H} & -.25408 & -1.11092 & 2.10714 \\ \mathrm{H} & 1.85978 & 1.11411 & 2.25690 \\ \mathrm{H} & 2.96625 & -1.68030 & -1.36998 \\ \mathrm{H} & 2.17210 & -2.25820 & .11605 \\ \mathrm{H} & 3.45711 & -1.04145 & .23307 \\ \mathrm{H} & 1.73052 & -.14186 & -2.93328 \\ \mathrm{H} & .11447 & .50473 & -2.55655 \\ \mathrm{H} & .41418 & -1.24154 & -2.45455 \\ \mathrm{H} & 2.37457 & 1.87525 & -1.57467 \\ \mathrm{H} & 2.98026 & 1.64702 & .09767 \\ \mathrm{H} & 1.36996 & 2.33291 & -.17984 \\ \mathrm{H} & 2.17286 & -.69300 & 2.35878\end{array}$

POMe $_{3}$ (B3LYP 6-31G*)

$\mathrm{E}\left(\mathrm{B} 3 \mathrm{LYP} 6-31 \mathrm{G}^{*}\right)=-536.35312$

$\mathrm{E}\left(\mathrm{B} 3 \mathrm{LYP} 6-311+\mathrm{G}^{* *}\right)=-536.44536$

$\mathrm{E}\left(\mathrm{B} 3 \mathrm{LYP} 6-31 \mathrm{G}^{*}(\mathrm{THF})\right)=$ $-536.37003$

$\mathrm{E}\left(\mathrm{B} 3 \mathrm{LYP} 6-311+\mathrm{G}^{* *}(\mathrm{THF})\right)=$ $-536.46544$

$\mathrm{E}\left(\mathrm{MP} 26-311+\mathrm{G}^{* *}\right)=-535.33484$

$\mathrm{E}\left(\mathrm{QCISD}(\mathrm{T}) 6-31 \mathrm{G}^{*}\right)=-535.22383$

$\mathrm{E}(\mathrm{G} 3(\mathrm{MP} 2))=-535.62360$

$$
\begin{array}{lccc}
\mathrm{O} & .52790 & -1.78282 & -.02968 \\
\mathrm{P} & -.00806 & -.38105 & -.03274 \\
\mathrm{C} & 1.28873 & .91571 & -.02416 \\
\mathrm{C} & -1.04731 & .01779 & -1.49019 \\
\mathrm{C} & -1.06645 & .01749 & 1.41104 \\
\mathrm{H} & 1.91428 & .79089 & .86520 \\
\mathrm{H} & .86451 & 1.92558 & -.02540 \\
\mathrm{H} & 1.92434 & .79296 & -.90670 \\
\mathrm{H} & -1.42556 & 1.04532 & -1.46097 \\
\mathrm{H} & -1.89371 & -.67515 & -1.52632 \\
\mathrm{H} & -. .45576 & -.12078 & -2.40056 \\
\mathrm{H} & -1.91440 & -.67404 & 1.43529 \\
\mathrm{H} & -1.44252 & 1.04564 & 1.37746 \\
\mathrm{H} & -. .48719 & -.12277 & 2.32904
\end{array}
$$

\section{POMe $_{3}($ B3L YP 6-31+G*)}

$\mathrm{E}(\mathrm{B} 3 \mathrm{LYP}$ 6-31+G*) $=-536.36423$

$\begin{array}{llll}\text { O } & .52942 & -1.78603 & -.03015\end{array}$

$\begin{array}{cccc}\mathrm{P} & -.00875 & -.37915 & -.03285 \\ \mathrm{C} & 1.29123 & .91681 & -.02368 \\ \mathrm{C} & -1.04878 & .01702 & -1.49235 \\ \mathrm{C} & -1.06744 & .01615 & 1.41340 \\ \mathrm{H} & 1.91543 & .79387 & .86754 \\ \mathrm{H} & .86359 & 1.92601 & -.02640 \\ \mathrm{H} & 1.92763 & .79436 & -.90629 \\ \mathrm{H} & -1.42339 & 1.04660 & -1.46154 \\ \mathrm{H} & -1.89805 & -.67331 & -1.52651 \\ \mathrm{H} & -.45773 & -.12297 & -2.40343 \\ \mathrm{H} & -1.91651 & -.67485 & 1.43718 \\ \mathrm{H} & -1.44255 & 1.04539 & 1.37753 \\ \mathrm{H} & -.48771 & -.12294 & 2.33185\end{array}$

POMe $_{3}($ B3LYP 6-311+G**)

$\mathrm{E}\left(\right.$ B3LYP $\left.6-311+\mathrm{G}^{* *}\right)=-536.44545$

$$
\begin{array}{lccc}
\mathrm{O} & .52426 & -1.77534 & -.02994 \\
\mathrm{P} & -.01038 & -.37464 & -.03288 \\
\mathrm{C} & 1.29257 & .91052 & -.02389 \\
\mathrm{C} & -1.04566 & .01137 & -1.49126 \\
\mathrm{C} & -1.06425 & .01069 & 1.41235 \\
\mathrm{H} & 1.91203 & .77892 & .86550 \\
\mathrm{H} & .87422 & 1.91995 & -.02721 \\
\mathrm{H} & 1.92471 & .77818 & -.90420 \\
\mathrm{H} & -1.42454 & 1.03598 & -1.46789 \\
\mathrm{H} & -1.88644 & -.68455 & -1.52062 \\
\mathrm{H} & -.44998 & -.13328 & -2.39475 \\
\mathrm{H} & -1.90578 & -.68470 & 1.43025 \\
\mathrm{H} & -1.44211 & 1.03559 & 1.38509 \\
\mathrm{H} & -.48029 & -.13518 & 2.32328
\end{array}
$$

\section{$\mathrm{POMe}_{3}$ (MP2 6-31G*)}

$\mathrm{E}\left(\mathrm{MP} 26-31 \mathrm{G}^{*}\right)=-535.15043$ $\mathrm{E}(\mathrm{QCISD}(\mathrm{T})$ 6-31G*) $=-535.22384$
$\begin{array}{llll}\text { O } & 0.53012 & -1.78521 & -0.02945\end{array}$
$\begin{array}{llll}\mathrm{P} & -0.00916 & -0.37817 & -0.03234\end{array}$
$\begin{array}{llll}\text { C } & 1.27151 & 0.91499 & -0.02355\end{array}$
$\begin{array}{llll}\text { C } & -1.04247 & 0.02497 & -1.47534\end{array}$
$\begin{array}{llll}\text { C }-1.06093 & 0.02430 & 1.39746\end{array}$
$\begin{array}{llll}\mathrm{H} & 1.89593 & 0.79910 & 0.86570\end{array}$
$\begin{array}{lllll}\mathrm{H} & 0.83691 & 1.91864 & -0.02637\end{array}$
$\begin{array}{llll}\mathrm{H} & 1.90794 & 0.79932 & -0.90430\end{array}$
H $-1.41264 \quad 1.05341-1.43676$
$\begin{array}{llll}\mathrm{H} & -1.89357 & -0.65938 & -1.51271\end{array}$
$\begin{array}{llll}\mathrm{H} & -0.45681 & -0.10940 & -2.38808\end{array}$
$\begin{array}{llll}\mathrm{H} & -1.91303 & -0.65934 & 1.42329\end{array}$
$\begin{array}{llll}\mathrm{H} & -1.42975 & 1.05307 & 1.35471\end{array}$
H $-0.48732 \quad-0.11106 \quad 2.31766$

\section{$\mathrm{POMe}_{3}(\mathrm{MP2}$ 6-31+G*)}

$\mathrm{E}\left(\mathrm{MP} 26-31+\mathrm{G}^{*}\right)=-535.16668$

$\begin{array}{llll}\text { O } & 0.52825 & -1.78043 & -0.02920\end{array}$ 


$\begin{array}{rrrr}\text { P } & -0.01300 & -0.36816 & -0.03231 \\ \text { C } & 1.27426 & 0.91726 & -0.02360 \\ \text { C } & -1.04436 & 0.02540 & -1.47829 \\ \text { C } & -1.06297 & 0.02471 & 1.40040 \\ \text { H } & 1.89872 & 0.79560 & 0.86624 \\ \text { H } & 0.84367 & 1.92369 & -0.02637 \\ \text { H } & 1.91051 & 0.79577 & -0.90508 \\ \text { H } & -1.41690 & 1.05415 & -1.44393 \\ \text { H } & -1.89323 & -0.66363 & -1.51342 \\ \text { H } & -0.45525 & -0.11361 & -2.38946 \\ \text { H } & -1.91258 & -0.66390 & 1.42394 \\ \text { H } & -1.43452 & 1.05367 & 1.36195 \\ \text { H } & -0.48585 & -0.11528 & 2.31905\end{array}$

\section{$\mathrm{POMe}_{3}(\mathrm{MP2}$ 6-311+G**)}

$\mathrm{E}\left(\mathrm{MP} 26-311+\mathrm{G}^{* *}\right)=-535.33532$

$\begin{array}{llll}\text { O } & 0.52343 & -1.76779 & -0.02939\end{array}$

$\begin{array}{llll}\mathrm{P} & -0.01303 & -0.36809 & -0.03234\end{array}$

$\begin{array}{llll}\text { C } & 1.27000 & 0.91612 & -0.02360\end{array}$

$\begin{array}{llll}\text { C } & -1.04256 & 0.02664 & -1.47447\end{array}$

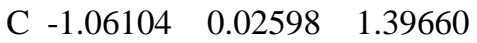

$\begin{array}{llll}\mathrm{H} & 1.89158 & 0.79047 & 0.86636\end{array}$

$\begin{array}{llll}\mathrm{H} & 0.83932 & 1.92133 & -0.02641\end{array}$

$\begin{array}{llll}\mathrm{H} & 1.90358 & 0.79063 & -0.90508\end{array}$

H $-1.41473 \quad 1.05435-1.43950$

H $-1.88848-0.66445-1.50618$

$\begin{array}{llll}\text { H } & -0.45055 & -0.11392 & -2.38219\end{array}$

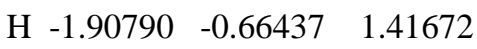

H $-1.43185 \quad 1.05405 \quad 1.35756$

H $-0.48102 \quad-0.11571 \quad 2.31185$

\section{POMe $_{3}($ B3LYP 6-31G*(THF))}

$\mathrm{E}(\mathrm{B} 3 \mathrm{LYP} 6-31 \mathrm{G} *(\mathrm{THF}))=-536.37016$

$\begin{array}{lccc}\mathrm{O} & .52510 & -1.78064 & -.02892 \\ \mathrm{P} & -.01407 & -.36495 & -.03262 \\ \mathrm{C} & 1.29996 & .90526 & -.02526 \\ \mathrm{C} & -1.04516 & .00547 & -1.49451 \\ \mathrm{C} & -1.06484 & .00538 & 1.41558 \\ \mathrm{H} & 1.92549 & .77851 & .86556 \\ \mathrm{H} & .87866 & 1.91546 & -.02659 \\ \mathrm{H} & 1.93347 & .77997 & -.91071 \\ \mathrm{H} & -1.42904 & 1.03000 & -1.46404 \\ \mathrm{H} & -1.88858 & -.69332 & -1.53225 \\ \mathrm{H} & -.44747 & -.12688 & -2.40331 \\ \mathrm{H} & -1.91227 & -.68913 & 1.43754 \\ \mathrm{H} & -1.44321 & 1.03185 & 1.38236 \\ \mathrm{H} & -.48237 & -.13318 & 2.33338\end{array}$

\section{$\mathrm{C}_{2} \mathrm{H}_{4}$ (B3LYP 6-31G*)}

$\mathrm{E}(\mathrm{B} 3 \mathrm{LYP}$ 6-31G*) $=-78.58755$

$\mathrm{E}\left(\mathrm{B} 3 \mathrm{LYP} 6-311+\mathrm{G}^{* *}\right)=-78.61556$

$\mathrm{E}\left(\mathrm{B} 3 \mathrm{LYP} 6-31 \mathrm{G}^{*}(\mathrm{THF})\right)=-78.58955$

\author{
$\mathrm{E}\left(\mathrm{B} 3 \mathrm{LYP} 6-311+\mathrm{G}^{* *}(\mathrm{THF})\right)=$ \\ $-78.61782$ \\ $\mathrm{E}\left(\mathrm{MP} 26-311+\mathrm{G}^{* *}\right)=-78.34620$ \\ $\mathrm{E}(\mathrm{QCISD}(\mathrm{T})$ 6-31G*) $=-78.32208$ \\ $\mathrm{E}(\mathrm{G} 3(\mathrm{MP} 2))=-78.43899$ \\ $\begin{array}{llll}\text { C } & .00000 & .00000 & .66556\end{array}$ \\ $\begin{array}{llll}\text { C } .00000 & .00000 & -.66556\end{array}$ \\ $\begin{array}{llll}\mathrm{H} & .00000 & .92392 & 1.23908\end{array}$ \\ H $.00000 \quad .92392 \quad-1.23908$ \\ H $.00000 \quad-.92392 \quad-1.23908$ \\ H .00000 $-.92392 \quad 1.23908$
}

\section{$\mathrm{C}_{2} \mathrm{H}_{4}$ (B3LYP 6-31+G*)}

$\mathrm{E}\left(\right.$ B3LYP $\left.6-31+\mathrm{G}^{*}\right)=-78.59328$

$\begin{array}{llll}\text { C } & .00000 & .00000 & .66782\end{array}$

$\begin{array}{llll}\text { C } & .00000 & .00000 & -.66782\end{array}$

$\begin{array}{llll}\mathrm{H} & .00000 & .92513 & 1.23971\end{array}$

H $.00000 \quad .92513 \quad-1.23971$

H $.00000 \quad-.92513 \quad-1.23971$

H $.00000 \quad-.92513 \quad 1.23971$

\section{$\mathrm{C}_{2} \mathrm{H}_{4}$ (B3LYP 6-311+G**)}

$\mathrm{E}\left(\right.$ B3LYP $\left.6-31+\mathrm{G}^{* *}\right)=-78.61556$

$\begin{array}{llll}\text { C } .00000 & .00000 & .66460\end{array}$

C .00000 $\quad .00000 \quad-.66460$

$\mathrm{H} \quad .00000 \quad .92318 \quad 1.23479$

H $.00000 \quad .92318 \quad-1.23479$

H $.00000 \quad-.92318-1.23479$

H $\begin{array}{lll}.00000 & -.92318 & 1.23479\end{array}$

\section{$\mathrm{C}_{2} \mathrm{H}_{4}$ (MP2 6-31G*)}

$\mathrm{E}\left(\mathrm{MP} 26-31 \mathrm{G}^{*}\right)=-78.28502$

$\mathrm{E}\left(\mathrm{QCISD}(\mathrm{T}) 6-31 \mathrm{G}^{*}\right)=-78.32215$

$\begin{array}{llll}\mathrm{C} & 2.11479 & 1.12736 & 0.00000 \\ \mathrm{C} & 0.77817 & 1.12736 & 0.00000 \\ \mathrm{H} & 2.68424 & 0.20380 & 0.00000 \\ \mathrm{H} & 0.20871 & 0.20380 & 0.00000 \\ \mathrm{H} & 0.20871 & 2.05092 & 0.00000 \\ \mathrm{H} & 2.68425 & 2.05092 & 0.00000\end{array}$

\section{$\mathrm{C}_{2} \mathrm{H}_{4}$ (MP2 6-31+G*)}

$\mathrm{E}\left(\mathrm{MP} 26-31+\mathrm{G}^{*}\right)=-78.29117$

$\begin{array}{llll}\mathrm{C} & 2.11662 & 1.12736 & 0.00000 \\ \mathrm{C} & 0.77633 & 1.12736 & 0.00000 \\ \mathrm{H} & 2.68498 & 0.20228 & 0.00000 \\ \mathrm{H} & 0.20798 & 0.20228 & 0.00000 \\ \mathrm{H} & 0.20797 & 2.05244 & 0.00000 \\ \mathrm{H} & 2.68498 & 2.05244 & 0.00000\end{array}$

$\mathrm{C}_{2} \mathrm{H}_{4}$ (MP2 6-311+G**)

$\mathrm{E}\left(\mathrm{MP} 26-311 \mathrm{G}^{* *}\right)=-78.34630$

$\begin{array}{llll}\text { C } & 2.11608 & 1.12736 & 0.00000\end{array}$

$\begin{array}{llll}\text { C } & 0.77688 & 1.12736 & 0.00000\end{array}$

$\begin{array}{llll}\mathrm{H} & 2.68143 & 0.20093 & 0.00000\end{array}$

$\begin{array}{llll}\mathrm{H} & 0.21152 & 0.20093 & 0.00000\end{array}$

$\begin{array}{llll}\mathrm{H} & 0.21152 & 2.05379 & 0.00000\end{array}$

$\begin{array}{llll}\mathrm{H} & 2.68144 & 2.05379 & 0.00000\end{array}$

\section{$\mathrm{C}_{2} \mathrm{H}_{4}$ (B3LYP 6-31G*(THF))}

$\mathrm{E}\left(\mathrm{B} 3 \mathrm{LYP} 6-31 \mathrm{G}^{*}(\mathrm{THF})\right)=-78.58949$

$\begin{array}{lll}\text { C } .00000 & .00000 & .66728\end{array}$

C .00000 $.00000 \quad-.66728$

H $.00000 \quad .92398 \quad 1.24320$

H $.00000 \quad .92398 \quad-1.24320$

H $.00000 \quad-.92398 \quad-1.24320$

$\begin{array}{llll}\text { H } & .00000 & -.92398 & 1.24320\end{array}$

Betaine (B3LYP 6-31G*(THF))

$\mathrm{E}\left(\mathrm{B} 3 \mathrm{LYP} 6-31 \mathrm{G}^{*}(\mathrm{THF})\right)=$ $-614.89190$

$\begin{array}{cccc}\mathrm{C} & .46031 & .27506 & -.18705 \\ \mathrm{O} & -.24035 & -.39243 & -1.08711 \\ \mathrm{P} & 2.35439 & .06333 & 1.97017 \\ \mathrm{C} & 1.16837 & -.71500 & .84262 \\ \mathrm{H} & 1.29752 & .92742 & -.59009 \\ \mathrm{C} & 1.63858 & 1.58529 & 2.66946 \\ \mathrm{C} & 2.81649 & -1.03323 & 3.35161 \\ \mathrm{C} & 3.86183 & .50867 & 1.04960 \\ \mathrm{H} & -.11935 & .98943 & .46951 \\ \mathrm{H} & .40230 & -1.23057 & 1.43351 \\ \mathrm{H} & 2.35130 & 2.05776 & 3.35268 \\ \mathrm{H} & .71930 & 1.34528 & 3.21271 \\ \mathrm{H} & 1.39636 & 2.28039 & 1.86006 \\ \mathrm{H} & 3.53448 & -.53731 & 4.01363 \\ \mathrm{H} & 3.26058 & -1.95328 & 2.95791 \\ \mathrm{H} & 1.91783 & -1.29096 & 3.92169 \\ \mathrm{H} & 4.57630 & 1.01598 & 1.70519 \\ \mathrm{H} & 3.59424 & 1.17091 & .22023 \\ \mathrm{H} & 4.32174 & -.39720 & .64151 \\ \mathrm{H} & 1.71057 & -1.47340 & .26523\end{array}$

\section{B. $\mathrm{Me}_{3} \mathrm{PCHMe}+$ MeCHO (reaction 1)}

\section{MeCHO (B3LYP 6-31G*)}

$\mathrm{E}\left(\right.$ B3LYP $\left.6-31 \mathrm{G}^{*}\right)=-153.83026$ $\mathrm{E}\left(\right.$ B3LYP $\left.6-31 \mathrm{G}^{*}(\mathrm{THF})\right)=$ 


$\begin{array}{cccc}-153.83730 & & \\ & & & \\ \mathrm{C} & 1.01808 & 1.27565 & -.36987 \\ \mathrm{H} & 1.32434 & .71661 & .54391 \\ \mathrm{O} & 1.01492 & 2.48613 & -.37691 \\ \mathrm{C} & .62687 & .41012 & -1.54084 \\ \mathrm{H} & .34009 & 1.02872 & -2.39427 \\ \mathrm{H} & 1.46483 & -.24395 & -1.81574 \\ \mathrm{H} & -.20584 & -.24638 & -1.25599\end{array}$

\section{MeCHO (B3LYP 6-31G*(THF))}

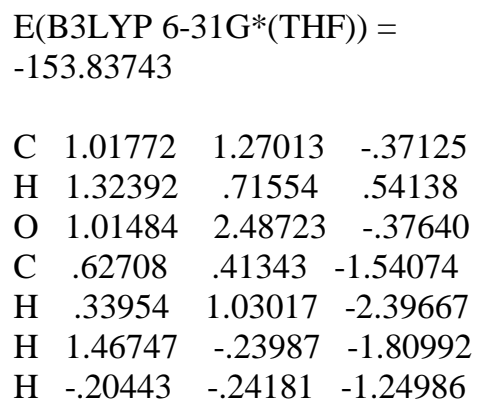

\section{Me $_{3}$ PCHMe (B3LYP 6-31G*)}

$\mathrm{E}\left(\mathrm{B} 3 \mathrm{LYP} 6-31 \mathrm{G}^{*}\right)=-539.66227$

$\mathrm{E}(\mathrm{B} 3 \mathrm{LYP} 6-31 \mathrm{G} *(\mathrm{THF}))=$ $-539.66891$

$\begin{array}{cccc}\mathrm{C} & .31834 & -.00373 & .38760 \\ \mathrm{P} & .42335 & -.13717 & 2.05918 \\ \mathrm{H} & .02011 & .98482 & .03978 \\ \mathrm{C} & 1.05718 & -.88918 & -.59289 \\ \mathrm{H} & 1.27204 & -1.88065 & -.17032 \\ \mathrm{H} & .44913 & -1.07032 & -1.49147 \\ \mathrm{H} & 2.02244 & -.48702 & -.94612 \\ \mathrm{C} & -.89276 & .87080 & 2.84677 \\ \mathrm{H} & -.78735 & .87517 & 3.93614 \\ \mathrm{H} & -.82091 & 1.90150 & 2.48536 \\ \mathrm{H} & -1.87309 & .47442 & 2.57220 \\ \mathrm{C} & 1.93075 & .36363 & 3.06862 \\ \mathrm{H} & 1.80948 & .18496 & 4.14481 \\ \mathrm{H} & 2.80176 & -.19879 & 2.71480 \\ \mathrm{H} & 2.12828 & 1.42831 & 2.90393 \\ \mathrm{C} & .19638 & -1.86976 & 2.64260 \\ \mathrm{H} & .28205 & -1.93368 & 3.73214 \\ \mathrm{H} & -.78470 & -2.23207 & 2.32550 \\ \mathrm{H} & .96260 & -2.51313 & 2.19890\end{array}$

$\begin{array}{cccc}\mathrm{H} & 1.26303 & -1.88063 & -.18115 \\ \mathrm{H} & .57919 & -1.00271 & -1.53441 \\ \mathrm{H} & 2.10137 & -.47674 & -.82939 \\ \mathrm{C} & -.89107 & .86946 & 2.86440 \\ \mathrm{H} & -.77224 & .86687 & 3.95267 \\ \mathrm{H} & -.81624 & 1.90277 & 2.50802 \\ \mathrm{H} & -1.87833 & .47906 & 2.60358 \\ \mathrm{C} & 1.94516 & .37025 & 3.00499 \\ \mathrm{H} & 1.85589 & .21373 & 4.08709 \\ \mathrm{H} & 2.80529 & -.19948 & 2.63503 \\ \mathrm{H} & 2.13422 & 1.43217 & 2.81123 \\ \mathrm{C} & .18626 & -1.87140 & 2.65762 \\ \mathrm{H} & .30695 & -1.92923 & 3.74439 \\ \mathrm{H} & -.80968 & -2.22793 & 2.38075 \\ \mathrm{H} & .93191 & -2.52690 & 2.19507\end{array}$

\section{Cis TS1 (B3LYP 6-31G*)}

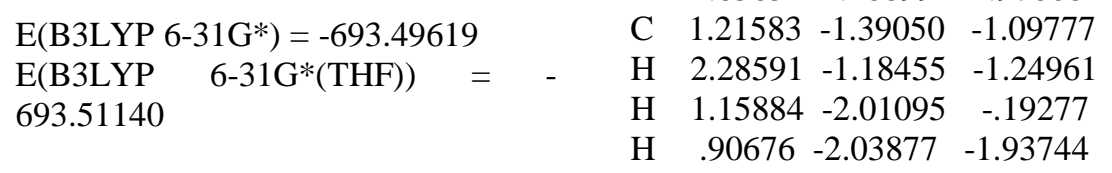

\begin{abstract}
$\begin{array}{llll}\mathrm{H} & 1.97609 & 2.22153 & -.19489\end{array}$
H $\quad .45503 \quad .57993 \quad-1.79755$

$\begin{array}{llll}\text { C } & -1.65218 & 1.68267 & -.04601\end{array}$

$\begin{array}{lllll}\mathrm{H} & -2.63691 & 1.73366 & .42979\end{array}$

$\begin{array}{lllll}\mathrm{H} & -.93158 & 2.27366 & .52543\end{array}$

$\begin{array}{llll}\mathrm{H} & -1.73053 & 2.08923 & -1.05980\end{array}$

$\begin{array}{llll}\text { C } & -2.56410 & -.98383 & -.72802\end{array}$

$\begin{array}{llll}\mathrm{H} & -3.43297 & -.83754 & -.07410\end{array}$

$\begin{array}{llll}\mathrm{H} & -2.81379 & -.64092 & -1.73841\end{array}$

$\begin{array}{llll}\mathrm{H} & -2.33440 & -2.05447 & -.77733\end{array}$

$\begin{array}{llll}\text { C } & -.90405 & -.63621 & 1.60796\end{array}$

$\begin{array}{llll}\mathrm{H} & -1.87819 & -.65501 & 2.10765\end{array}$

$\begin{array}{llll}\mathrm{H} & -.48641 & -1.64841 & 1.62347\end{array}$

$\begin{array}{llll}\mathrm{H} & -.22660 & .03596 & 2.14161\end{array}$

$\begin{array}{llll}\text { C } & 3.08862 & .81066 & 1.11432\end{array}$

$\begin{array}{llll}\mathrm{H} & 3.32015 & .18693 & .24735\end{array}$

$\begin{array}{llll}\mathrm{H} & 3.99399 & 1.38826 & 1.35796\end{array}$

$\begin{array}{llll}\mathrm{H} & 2.83854 & .18899 & 1.97868\end{array}$

$\begin{array}{llll}\text { C } & 1.21583 & -1.39050 & -1.09777\end{array}$

$\begin{array}{lllll}\mathrm{H} & 2.28591 & -1.18455 & -1.24961\end{array}$

H $\quad .90676 \quad-2.03877 \quad-1.93744$
\end{abstract}
$\mathrm{Me}_{3} \mathrm{PCHMe}$ 31G*(THF))
(B3LYP
$\mathrm{E}($ B3LYP 6-31G*(THF) $)=-539.66956$
6- Cis TS1 (B3LYP 6-31G*(THF))
$\mathrm{E}(\mathrm{B} 3 \mathrm{LYP} 6-31 \mathrm{G} *(\mathrm{THF}))=$ $-693.50966$

$$
\begin{array}{lrll}
\mathrm{O} & 1.20481 & 2.20093 & 1.67763 \\
\mathrm{C} & 1.97824 & 1.78621 & .81976 \\
\mathrm{P} & -1.06482 & -.04792 & -.12375 \\
\mathrm{C} & .43065 & -.10201 & -.94507
\end{array}
$$

$\begin{array}{cccc}\text { O } & 1.01088 & 1.58275 & 1.69720 \\ \text { C } & 1.59146 & 1.44205 & .58089 \\ \text { P } & -.99893 & .09847 & -.03768 \\ \text { C } & .64140 & .03917 & -.66695 \\ \text { H } & 1.44377 & 2.22988 & -.19241 \\ \text { H } & .68139 & .53515 & -1.64284 \\ \text { C } & -1.55984 & 1.82848 & .07803 \\ \text { H } & -2.59761 & 1.87526 & .42214 \\ \text { H } & -.88709 & 2.30430 & .80163 \\ \text { H } & -1.48125 & 2.31931 & -.89667 \\ \text { C } & -2.31648 & -.80119 & -.96917 \\ \text { H } & -3.27800 & -.73951 & -.44639 \\ \text { H } & -2.42581 & -.36769 & -1.96825 \\ \text { H } & -2.03997 & -1.85467 & -1.07646 \\ \text { C } & -1.01407 & -.58980 & 1.64917 \\ \text { H } & -1.99343 & -.44726 & 2.11603 \\ \text { H } & -.77993 & -1.65797 & 1.62590 \\ \text { H } & -.23687 & -.03677 & 2.19253 \\ \text { C } & 3.01617 & .89690 & .55517 \\ \text { H } & 3.39402 & .74800 & -.46288 \\ \text { H } & 3.66169 & 1.62916 & 1.05896 \\ \text { H } & 3.08710 & -.04207 & 1.11374 \\ \text { C } & 1.28036 & -1.34778 & -.68344 \\ \text { H } & 2.30670 & -1.27498 & -1.05847 \\ \text { H } & 1.34755 & -1.77373 & .32574 \\ \text { H } & .76647 & -2.08932 & -1.31709\end{array}$

\section{Cis TS1b (B3LYP 6-31G*)} E(B3LYP 6-31G*) $=-693.49575$
E(B3LYP 6-31G*(THF) $)=$
-693.51162

$$
\begin{array}{cccc}
\mathrm{O} & .85580 & 1.78558 & 1.59380 \\
\mathrm{C} & 1.61622 & 1.36106 & .66302 \\
\mathrm{P} & -1.01879 & .14449 & -.03441 \\
\mathrm{C} & .64538 & .09264 & -.63216 \\
\mathrm{H} & 1.86293 & 2.06136 & -.16617 \\
\mathrm{H} & .68118 & .65451 & -1.57287 \\
\mathrm{C} & -1.62488 & 1.86050 & -.04190 \\
\mathrm{H} & -2.63390 & 1.91711 & .37793 \\
\mathrm{H} & -.90679 & 2.41521 & .57606 \\
\mathrm{H} & -1.63560 & 2.24976 & -1.06503 \\
\mathrm{C} & -2.25723 & -.84771 & -.97515 \\
\mathrm{H} & -3.24301 & -.79244 & -.49961 \\
\mathrm{H} & -2.33380 & -.46748 & -1.99857 \\
\mathrm{H} & -1.94510 & -1.89578 & -1.01680 \\
\mathrm{C} & -1.07955 & -.46388 & 1.68125 \\
\mathrm{H} & -2.09242 & -.38090 & 2.08800 \\
\mathrm{H} & -.76065 & -1.50948 & 1.72202 \\
\mathrm{H} & -. .37434 & .17395 & 2.22973 \\
\mathrm{C} & 2.83683 & .53192 & 1.06224 \\
\mathrm{H} & 3.41735 & .18184 & .20131 \\
\mathrm{H} & 3.48466 & 1.17274 & 1.67525 \\
\mathrm{H} & 2.54897 & -.32625 & 1.67955 \\
\mathrm{C} & 1.22687 & -1.31192 & -.79216 \\
\mathrm{H} & 2.25350 & -1.24355 & -1.16763 \\
\mathrm{H} & 1.27952 & -1.84437 & .16594 \\
\mathrm{H} & .67763 & -1.95921 & -1.49548
\end{array}
$$

Cis TS1c (B3LYP 6-31G*) 


$\begin{array}{lccc}\text { E(B3LYP 6-31G*) }=-693.49444 \\ \text { E(B3LYP 6-31G*(THF)) } \\ -693.51378 \\ \text { O } & & \\ \text { O } & 1.76385 & .70718 & 1.68938 \\ \text { C } & 1.62321 & 1.38289 & .63018 \\ \text { P } & -1.03473 & .15687 & -.06078 \\ \text { C } & .58752 & .28018 & -.72560 \\ \text { H } & .92896 & 2.26106 & .63386 \\ \text { H } & .55807 & .82101 & -1.67688 \\ \text { C } & -1.81248 & 1.81079 & .00427 \\ \text { H } & -2.82235 & 1.74303 & .41984 \\ \text { H } & -1.20279 & 2.46934 & .62807 \\ \text { H } & -1.86895 & 2.23514 & -1.00285 \\ \text { C } & -2.26572 & -.89116 & -.95609 \\ \text { H } & -3.21398 & -.93650 & -.40801 \\ \text { H } & -2.44682 & -.48376 & -1.95559 \\ \text { H } & -1.87074 & -1.90622 & -1.06079 \\ \text { C } & -.96132 & -.49664 & 1.63721 \\ \text { H } & -1.89719 & -.29788 & 2.16864 \\ \text { H } & -.78747 & -1.57677 & 1.61043 \\ \text { H } & -.09109 & -.01585 & 2.11408 \\ \text { C } & 2.86849 & 1.70702 & -.20271 \\ \text { H } & 2.62590 & 2.18234 & -1.16108 \\ \text { H } & 3.49302 & 2.39812 & .37929 \\ \text { H } & 3.46052 & .80331 & -.37855 \\ \text { C } & 1.35837 & -1.03678 & -.75941 \\ \text { H } & 2.24403 & -.92738 & -1.39377 \\ \text { H } & 1.71019 & -1.26072 & .25747 \\ \text { H } & .80264 & -1.90651 & -1.14164\end{array}$

Cis TS1d (B3LYP 6-31G*(THF))

$\begin{array}{lccc}\text { E (B3LYP 6-31G*(THF) })= \\ \text {-693.50823 } & & \\ & & & \\ \text { O } & 2.18824 & .94850 & 1.64372 \\ \text { C } & 1.60834 & 1.79720 & .96572 \\ \text { P } & -1.19437 & .03797 & -.18405 \\ \text { C } & .33120 & .39028 & -.87643 \\ \mathrm{H} & .61616 & 2.17573 & 1.27133 \\ \mathrm{H} & .27684 & 1.11773 & -1.68806 \\ \mathrm{C} & -2.12503 & 1.58919 & .10371 \\ \mathrm{H} & -3.14710 & 1.37027 & .42868 \\ \mathrm{H} & -1.62672 & 2.19373 & .86652 \\ \mathrm{H} & -2.16605 & 2.16535 & -.82695 \\ \mathrm{C} & -2.44462 & -1.03910 & -1.04791 \\ \mathrm{H} & -3.34269 & -1.19319 & -.43668 \\ \mathrm{H} & -2.73127 & -.56957 & -1.99561 \\ \mathrm{H} & -1.99347 & -2.01299 & -1.26851 \\ \mathrm{C} & -.99169 & -.80749 & 1.43147 \\ \mathrm{H} & -1.94539 & -.84205 & 1.96775 \\ \mathrm{H} & -.64987 & -1.83603 & 1.27509 \\ \mathrm{H} & -.23976 & -.28007 & 2.02477 \\ \mathrm{C} & 2.31608 & 2.62128 & -.08635 \\ \mathrm{H} & 1.60999 & 3.10727 & -.76383 \\ \mathrm{H} & 2.90171 & 3.40085 & .42514 \\ \mathrm{H} & 3.01831 & 2.00375 & -.65609\end{array}$

$$
\begin{array}{cccc}
\mathrm{C} & 1.38588 & -.69175 & -1.01117 \\
\mathrm{H} & 2.39507 & -.28312 & -.85045 \\
\mathrm{H} & 1.26591 & -1.47413 & -.25138 \\
\mathrm{H} & 1.39552 & -1.20292 & -1.98724
\end{array}
$$

\section{Trans TS1 (B3LYP 6-31G*)}

$$
\begin{aligned}
& \mathrm{E}(\text { B3LYP 6-31G*) }=-693.49928 \\
& \mathrm{E}(\text { B3LYP 6-31G*(THF) })= \\
& -693.51413
\end{aligned}
$$

$\begin{array}{cccc}\mathrm{O} & 1.22198 & 1.50618 & 1.55635 \\ \mathrm{C} & 1.83884 & .59577 & .93281 \\ \mathrm{P} & -.91994 & .20781 & -.23693 \\ \mathrm{C} & .70808 & -.32094 & -.59743 \\ \mathrm{H} & 1.96586 & -.39483 & 1.42580 \\ \mathrm{H} & 1.05461 & .17181 & -1.51040 \\ \mathrm{C} & 3.05430 & .97804 & .08733 \\ \mathrm{H} & 3.43096 & .14705 & -.52024 \\ \mathrm{H} & 2.81253 & 1.82572 & -.56283 \\ \mathrm{H} & 3.85728 & 1.30105 & .76453 \\ \mathrm{C} & 1.01322 & -1.81051 & -.55621 \\ \mathrm{H} & 2.10109 & -1.95983 & -.53222 \\ \mathrm{H} & .62143 & -2.28094 & .35549 \\ \mathrm{H} & .62937 & -2.38600 & -1.41230 \\ \mathrm{C} & -.96938 & 2.01530 & -.43469 \\ \mathrm{H} & -1.94656 & 2.41641 & -.14911 \\ \mathrm{H} & -.18076 & 2.40005 & .22585 \\ \mathrm{H} & -.75807 & 2.28324 & -1.47462 \\ \mathrm{C} & -2.32430 & -.47746 & -1.22464 \\ \mathrm{H} & -3.28136 & -.06718 & -.88210 \\ \mathrm{H} & -2.18726 & -.23044 & -2.28188 \\ \mathrm{H} & -2.35140 & -1.56717 & -1.12369 \\ \mathrm{C} & -1.33227 & -.15714 & 1.50303 \\ \mathrm{H} & -2.33358 & .20975 & 1.74874 \\ \mathrm{H} & -1.29226 & -1.23508 & 1.68625 \\ \mathrm{H} & -.56724 & .35823 & 2.09719\end{array}$

\section{Trans TS1 (B3LYP 6- 31G*(THF))}

$$
\begin{aligned}
& \mathrm{E}(\mathrm{B} 3 \mathrm{LYP} 6-31 \mathrm{G} *(\mathrm{THF}))= \\
& -693.50966 \\
& \begin{array}{llll}
\text { O } & 1.43737 & 1.62878 & 1.82567
\end{array} \\
& \begin{array}{llll}
\text { C } & 2.16333 & .86104 & 1.19850
\end{array} \\
& \begin{array}{llll}
\mathrm{P} & -.96063 & .10650 & -.33038
\end{array} \\
& \text { C } \quad \begin{array}{llll}
.55205 & -.55141 & -.76219
\end{array} \\
& \begin{array}{llll}
\mathrm{H} & 2.26683 & -.19337 & 1.50336
\end{array} \\
& \begin{array}{llll}
\mathrm{H} & 1.03031 & -.02244 & -1.58641
\end{array} \\
& \begin{array}{llll}
\text { C } & 3.13739 & 1.32656 & .14607
\end{array} \\
& \begin{array}{llll}
\mathrm{H} & 3.29015 & .56367 & -.62127
\end{array} \\
& \begin{array}{llll}
\text { H } & 2.79859 & 2.26178 & -.30951
\end{array} \\
& \begin{array}{llll}
\mathrm{H} & 4.10502 & 1.51464 & .63688
\end{array} \\
& \begin{array}{llll}
\text { C } & .84277 & -2.03558 & -.62733
\end{array} \\
& \begin{array}{llll}
\mathrm{H} & 1.84435 & -2.24178 & -.21760
\end{array} \\
& \text { H } \quad .13953 \quad-2.52885 \quad .05790 \\
& \text { H } \quad .77801 \quad-2.59482-1.57575
\end{aligned}
$$

$$
\begin{array}{cccc}
\text { C } & -.91185 & 1.92173 & -.54091 \\
\text { H } & -1.90277 & 2.35650 & -.37597 \\
\text { H } & -.19908 & 2.33950 & .17468 \\
\text { H } & -.58768 & 2.16241 & -1.55875 \\
\text { C } & -2.52821 & -.40207 & -1.20491 \\
\text { H } & -3.41094 & .09477 & -.78338 \\
\text { H } & -2.44292 & -.14738 & -2.26717 \\
\text { H } & -2.66158 & -1.48678 & -1.12201 \\
\text { C } & -1.37543 & -.21826 & 1.42907 \\
\text { H } & -2.36654 & .17482 & 1.67911 \\
\text { H } & -1.36801 & -1.29630 & 1.62336 \\
\text { H } & -.61546 & .26317 & 2.05023
\end{array}
$$

\section{Cis OP1 (B3LYP 6-31G*)}

$\mathrm{E}(\mathrm{B} 3 \mathrm{LYP}$ 6-31G*) $=-693.53465$ $\mathrm{E}(\mathrm{B} 3 \mathrm{LYP} 6-31 \mathrm{G} *(\mathrm{THF}))=$ $-693.54195$

$\begin{array}{cccc}\mathrm{O} & -.00084 & -.27823 & .11430 \\ \mathrm{C} & .05453 & -.03193 & 1.50707 \\ \mathrm{C} & 1.58983 & .09671 & 1.65482 \\ \mathrm{P} & 1.78595 & .04146 & -.22671 \\ \mathrm{C} & 1.37131 & 1.49471 & -1.28775 \\ \mathrm{C} & 1.85631 & -1.53977 & -1.18157 \\ \mathrm{C} & 3.67615 & .33837 & -.25357 \\ \mathrm{H} & -.32525 & -.90251 & 2.06818 \\ \mathrm{H} & 2.01242 & -.84930 & 2.02338 \\ \mathrm{H} & 2.16584 & 1.71483 & -2.00684 \\ \mathrm{H} & 1.23810 & 2.37025 & -.64268 \\ \mathrm{H} & .42423 & 1.30762 & -1.79649 \\ \mathrm{H} & 2.69236 & -1.56125 & -1.88619 \\ \mathrm{H} & .90558 & -1.68576 & -1.69729 \\ \mathrm{H} & 1.97769 & -2.36398 & -.46993 \\ \mathrm{H} & 3.93057 & 1.31462 & .17596 \\ \mathrm{H} & 4.07251 & .31091 & -1.27585 \\ \mathrm{H} & 4.20034 & -.42925 & .33082 \\ \mathrm{C} & -.77161 & 1.18978 & 1.91219 \\ \mathrm{H} & -.72824 & 1.37093 & 2.99392 \\ \mathrm{H} & -.42979 & 2.09454 & 1.39628 \\ \mathrm{H} & -1.81763 & 1.02506 & 1.63320 \\ \mathrm{C} & 2.19901 & 1.24795 & 2.45350 \\ \mathrm{H} & 3.29219 & 1.19484 & 2.45932 \\ \mathrm{H} & 1.91177 & 2.22868 & 2.05902 \\ \mathrm{H} & 1.86244 & 1.20482 & 3.49703\end{array}$

\section{Cis OP1 (B3LYP 6-31G*(THF))}

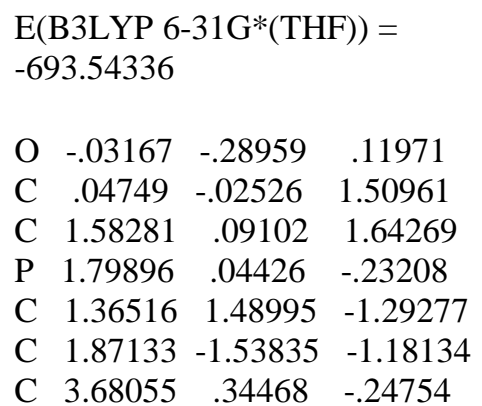




\begin{abstract}
$\begin{array}{llll}\mathrm{H} & -.32930 & -.88411 & 2.09124\end{array}$
$\begin{array}{llll}\mathrm{H} & 1.99740 & -.86163 & 2.00242\end{array}$

H $2.17119 \quad 1.72712 \quad-1.99385$

$\begin{array}{llll}\mathrm{H} & 1.19719 & 2.36272 & -.65178\end{array}$

$\mathrm{H} \quad .43785 \quad 1.28510 \quad-1.83088$

H $2.70060 \quad-1.54275-1.89484$

H $\quad .92268$ - $1.70543 \quad-1.69555$

H $2.02004 \quad-2.35862 \quad-.46953$

$\begin{array}{llll}\mathrm{H} & 3.92722 & 1.32195 & .18384\end{array}$

$\begin{array}{llll}\mathrm{H} & 4.07955 & .31549 & -1.26854\end{array}$

$\begin{array}{llll}\text { H } & 4.19696 & -.42389 & .34221\end{array}$

$\begin{array}{llll}\text { C } & -.76331 & 1.20629 & 1.91876\end{array}$

H $\quad-.69362 \quad 1.39586 \quad 2.99746$

H $\quad-.42721 \quad 2.10422 \quad 1.38715$

H $-1.81905 \quad 1.04710 \quad 1.67010$

$\begin{array}{llll}\text { C } & 2.19999 & 1.23222 & 2.45092\end{array}$

H $3.29243 \quad 1.16799 \quad 2.46520$

$\begin{array}{llll}\mathrm{H} & 1.92427 & 2.21821 & 2.06103\end{array}$

H $1.85495 \quad 1.18209 \quad 3.49158$
\end{abstract}

Trans OP1 (B3LYP 6-31G*)

E(B3LYP 6-31G*) $=-693.53707$

$\mathrm{E}(\mathrm{B} 3 \mathrm{LYP} 6-31 \mathrm{G} *(\mathrm{THF}))=$ $-693.54419$

$\begin{array}{lccc}\text { O } & -.18381 & .01175 & -.32129 \\ \text { C } & .31192 & .06649 & 1.00245 \\ \text { C } & 1.74930 & .52322 & .69247 \\ \text { P } & 1.46467 & .17094 & -1.14265 \\ \text { C } & 1.38939 & -1.58480 & -1.70485 \\ \text { C } & .70509 & 1.35146 & -2.34769 \\ \text { C } & 3.25936 & .49498 & -1.71795 \\ \text { H } & .33204 & -.93781 & 1.46888 \\ \text { H } & 1.83127 & 1.61607 & .79204 \\ \text { H } & .37462 & -1.80063 & -2.04513 \\ \text { H } & 1.59095 & -2.23307 & -.84500 \\ \text { H } & 2.11829 & -1.80703 & -2.48909 \\ \text { H } & 1.25754 & 1.38207 & -3.29113 \\ \text { H } & .72044 & 2.35317 & -1.90383 \\ \text { H } & -. .33750 & 1.07446 & -2.51154 \\ \text { H } & 3.36697 & .34933 & -2.79965 \\ \text { H } & 3.96470 & -.17949 & -1.21840 \\ \text { H } & 3.56231 & 1.52471 & -1.48606 \\ \text { C } & -.51270 & .99572 & 1.88780 \\ \text { H } & -.09895 & 1.05134 & 2.90310 \\ \text { H } & -.53436 & 2.00580 & 1.46139 \\ \text { H } & -1.54505 & .63663 & 1.95834 \\ \text { C } & 2.90659 & -.14419 & 1.42984 \\ \text { H } & 3.87816 & .24376 & 1.10775 \\ \text { H } & 2.82057 & .03931 & 2.50863 \\ \text { H } & 2.90833 & -1.23123 & 1.28363\end{array}$

Trans OP1 (B3LYP 31G*(THF))

$\mathrm{E}(\mathrm{B} 3 \mathrm{LYP} 6-31 \mathrm{G} *(\mathrm{THF}))=$ $-693.54571$

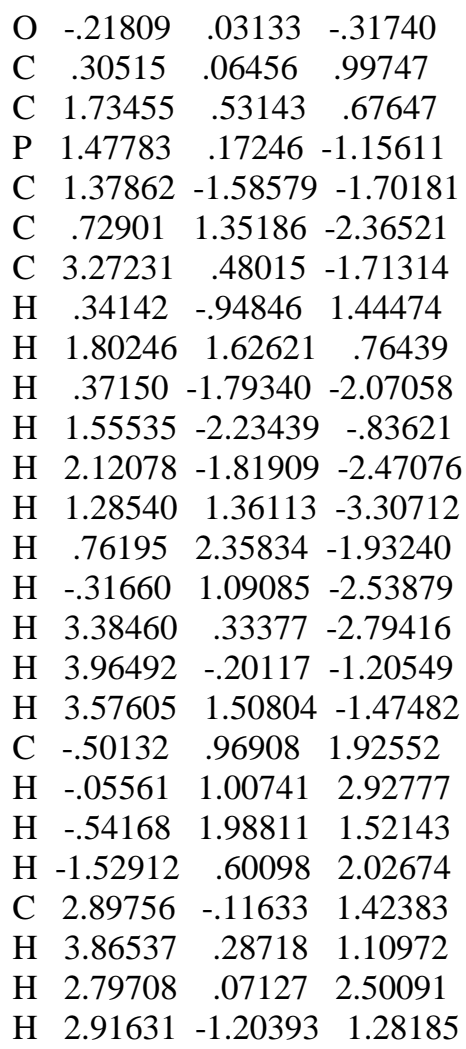

Betaine (B3LYP 6-31G*(THF))

$\mathrm{E}(\mathrm{B} 3 \mathrm{LYP} 6-31 \mathrm{G} *(\mathrm{THF}))=$ $-693.51825$

$\begin{array}{cccc}\mathrm{C} & .21132 & .95893 & -.21514 \\ \mathrm{C} & -1.33324 & 1.08629 & -.76540 \\ \mathrm{O} & -2.03095 & 1.94738 & -.04338 \\ \mathrm{H} & .82448 & 1.68940 & -.76322 \\ \mathrm{H} & -1.72878 & .02127 & -.73995 \\ \mathrm{P} & 1.00108 & -.65788 & -.51353 \\ \mathrm{C} & .23583 & -1.95812 & .51189 \\ \mathrm{H} & .39227 & -1.75026 & 1.57408 \\ \mathrm{H} & -.83914 & -1.99596 & .31093 \\ \mathrm{H} & .68447 & -2.92648 & .26773 \\ \mathrm{C} & .88315 & -1.21756 & -2.24564 \\ \mathrm{H} & -.16221 & -1.38899 & -2.51577 \\ \mathrm{H} & 1.31047 & -.46624 & -2.91628 \\ \mathrm{H} & 1.43915 & -2.15450 & -2.35670 \\ \mathrm{C} & 2.78112 & -.60712 & -.10538 \\ \mathrm{H} & 3.24822 & -1.57624 & -.31055 \\ \mathrm{H} & 3.26817 & .16378 & -.71160 \\ \mathrm{H} & 2.91267 & -.36066 & .95239 \\ \mathrm{C} & -1.27669 & 1.48031 & -2.26611 \\ \mathrm{H} & -2.30156 & 1.52707 & -2.65268 \\ \mathrm{H} & -.84518 & 2.48587 & -2.35220 \\ \mathrm{H} & -.70246 & .79959 & -2.90737 \\ \mathrm{C} & .26995 & 1.29465 & 1.28353 \\ \mathrm{H} & -.12399 & .48790 & 1.91244 \\ \mathrm{H} & 1.27864 & 1.54741 & 1.62799\end{array}$

$\begin{array}{llll}\mathrm{H} & -.40391 & 2.14735 & 1.40617\end{array}$

\section{Cis TS2 (B3LYP 6-31G*)}

$\mathrm{E}($ B3LYP 6-31G*) $=-693.49761$
E(B3LYP 6-31G*(THF) $)=$
-693.50487

$\begin{array}{llll}\text { O } & .54650 & .24170 & -.42727\end{array}$

$\begin{array}{llll}\text { C } & .35796 & .20561 & 1.36848\end{array}$

$\begin{array}{llll}\text { C } & 1.69087 & .22144 & 1.87054\end{array}$

$\begin{array}{llll}\text { P } & 2.11521 & .19783 & -.61561\end{array}$

$\begin{array}{llll}\mathrm{H} & -.14416 & -.76156 & 1.36166\end{array}$

$\begin{array}{llll}\text { H } & 2.04229 & -.74540 & 2.23545\end{array}$

$\begin{array}{llll}\text { C } & -.59477 & 1.36247 & 1.54985\end{array}$

$\begin{array}{llll}\mathrm{H} & -.92259 & 1.39889 & 2.59797\end{array}$

H $\quad-.11566 \quad 2.31813 \quad 1.31855$

$\begin{array}{llll}\mathrm{H} & -1.47715 & 1.25856 & .91276\end{array}$

$\begin{array}{llll}\text { C } & 2.22432 & 1.39860 & 2.66709\end{array}$

H $3.31425 \quad 1.331662 .78664$

$\begin{array}{llll}\text { H } & 2.02397 & 2.36566 & 2.18860\end{array}$

$\begin{array}{llll}\mathrm{H} & 1.80692 & 1.46511 & 3.68659\end{array}$

$\begin{array}{llll}\text { C } & 3.19761 & 1.66712 & -.36319\end{array}$

H $3.79424 \quad 1.86286-1.26206$

$\begin{array}{llll}\mathrm{H} & 2.56680 & 2.53905 & -.16872\end{array}$

$\begin{array}{lllll}\mathrm{H} & 3.85648 & 1.52437 & .49413\end{array}$

$\begin{array}{llll}\text { C } & 2.08394 & .17407 & -2.47328\end{array}$

$\begin{array}{llll}\text { H } & 3.09918 & .15196 & -2.88858\end{array}$

$\begin{array}{lllll}\mathrm{H} & 1.53448 & -.70146 & -2.83098\end{array}$

$\begin{array}{lllll}\mathrm{H} & 1.57275 & 1.06707 & -2.84594\end{array}$

$\begin{array}{llll}\text { C } 3.08097 & -1.33911 & -.30116\end{array}$

H $3.63165 \quad-1.64028$ - 1.20084

$\begin{array}{lllll}\mathrm{H} & 3.77078 & -1.19830 & .53177\end{array}$

$\begin{array}{llll}\text { H } & 2.37978 & -2.13671 & -.03747\end{array}$

\section{Cis TS2 (B3LYP 6-31G*(THF))}

$\mathrm{E}(\mathrm{B} 3 \mathrm{LYP} 6-31 \mathrm{G} *(\mathrm{THF}))=$ $-693.50435$
$\begin{array}{llll}\text { O } & .55191 & .29398 & -.42292\end{array}$
$\begin{array}{llll}\text { C } & .36937 & .18268 & 1.35847\end{array}$
$\begin{array}{llll}\mathrm{C} & 1.70516 & .24440 & 1.85400\end{array}$
$\begin{array}{llll}\text { P } & 2.13193 & .20651 & -.61545\end{array}$
$\begin{array}{llll}\mathrm{H} & -.08942 & -.80623 & 1.33867\end{array}$
$\begin{array}{lllll}\mathrm{H} & 2.09564 & -.70889 & 2.21245\end{array}$
C $\quad-.63472 \quad 1.28672 \quad 1.59815$
$\mathrm{H} \quad-.94636 \quad 1.26668 \quad 2.65196$
$\mathrm{H} \quad-.20719 \quad 2.27376 \quad 1.39733$
$\begin{array}{llll}\mathrm{H} & -1.52832 & 1.16612 & .97795\end{array}$
$\begin{array}{llll}\text { C } & 2.19291 & 1.44680 & 2.64159\end{array}$
H $3.27693 \quad 1.39342 \quad 2.80760$
$\begin{array}{llll}\mathrm{H} & 2.00520 & 2.39749 & 2.12642\end{array}$
$\begin{array}{llll}\mathrm{H} & 1.73047 & 1.54143 & 3.64058\end{array}$
$\begin{array}{llll}\text { C } & 3.25783 & 1.64178 & -.36448\end{array}$
H $3.88542 \quad 1.78965-1.25135$
$\begin{array}{llll}\mathrm{H} & 2.65455 & 2.54294 & -.21564\end{array}$
$\begin{array}{llll}\text { H } & 3.88686 & 1.49760 & .51476\end{array}$ 


\begin{abstract}
$\begin{array}{llll}\text { C } & 2.09226 & .19451 & -2.47440\end{array}$
$\begin{array}{llll}\mathrm{H} & 3.10670 & .11283 & -2.88353\end{array}$

$\begin{array}{lllll}\mathrm{H} & 1.49852 & -.65088 & -2.83644\end{array}$

H $1.63863 \quad 1.11732-2.85101$

$\begin{array}{llll}\text { C } & 3.02111 & -1.37574 & -.30414\end{array}$

H $3.51009-1.72790 \quad-1.22022$

H $3.76126-1.25017 \quad .48856$

H $2.29567 \quad-2.12982 \quad .01653$
\end{abstract}

\section{Trans TS2 (B3LYP 6-31G*)}

$\mathrm{E}($ B3LYP 6-31G*) $=-693.49907$

$\mathrm{E}(\mathrm{B} 3 \mathrm{LYP} 6-31 \mathrm{G} *(\mathrm{THF}))=$ $-693.50601$

\section{$\begin{array}{lllll}\text { O } & .56447 & .29770 & -.48101\end{array}$ \\ C $\quad \begin{array}{llll}.57276 & .14762 & 1.31299\end{array}$ \\ $\begin{array}{llll}\text { C } & 1.94558 & .10876 & 1.67629\end{array}$ \\ $\begin{array}{llll}\text { P } & 2.09997 & .21882 & -.85022\end{array}$ \\ $\begin{array}{llll}\mathrm{H} & .04826 & 1.08946 & 1.48191\end{array}$ \\ $\begin{array}{llll}\text { H } & 2.30989 & -.87466 & 1.98443\end{array}$ \\ C $-.31266-1.06629 \quad 1.46331$ \\ H $\quad-.59869-1.17055 \quad 2.51857$ \\ H $-1.22290 \quad-.99107 \quad .86196$ \\ H $\quad .22206 \quad-1.97741 \quad 1.17293$ \\ C $2.55972 \quad 1.266892 .44071$ \\ H $3.65586 \quad 1.286902 .35389$ \\ H $2.19605 \quad 2.235302 .07096$ \\ H $2.34286 \quad 1.24182 \quad 3.52225$ \\ $\begin{array}{llll}\text { C } & 3.21473 & 1.67218 & -.66726\end{array}$ \\ H $3.76996 \quad 1.83763 \quad-1.59803$ \\ $\begin{array}{llll}\text { H } & 2.59690 & 2.55411 & -.47253\end{array}$ \\ H $3.91108 \quad 1.54926 \quad .16169$ \\ $\begin{array}{llll}\text { C } & 1.85994 & .26008 & -2.69175\end{array}$ \\ H $2.81961 \quad .21507 \quad-3.22125$ \\ H $1.24433 \quad-.58805-3.00530$ \\ H $1.33998 \quad 1.17823 \quad-2.98063$ \\ $\begin{array}{llll}\text { C } & 3.08053 & -1.33646 & -.70366\end{array}$ \\ H $3.53483-1.60033-1.66681$ \\ $\begin{array}{llll}\text { H } & 3.85152 & -1.23069 & .06072\end{array}$ \\ $\begin{array}{llll}\text { H } & 2.40801 & -2.14451 & -.40213\end{array}$}

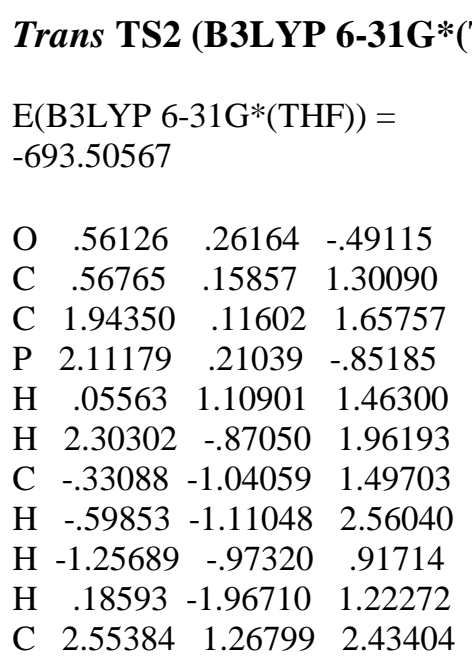

$\begin{array}{cccc}\mathrm{H} & 3.64944 & 1.29675 & 2.34658 \\ \mathrm{H} & 2.18334 & 2.23717 & 2.07439 \\ \mathrm{H} & 2.33595 & 1.23160 & 3.51626 \\ \mathrm{C} & 3.19551 & 1.68324 & -.64781 \\ \mathrm{H} & 3.71474 & 1.90113 & -1.58844 \\ \mathrm{H} & 2.56958 & 2.54421 & -.39167 \\ \mathrm{H} & 3.92511 & 1.53613 & .14864 \\ \mathrm{C} & 1.87759 & .26642 & -2.69414 \\ \mathrm{H} & 2.84558 & .24882 & -3.20946 \\ \mathrm{H} & 1.28627 & -.59113 & -3.03017 \\ \mathrm{H} & 1.34663 & 1.18074 & -2.97841 \\ \mathrm{C} & 3.09158 & -1.34319 & -.71053 \\ \mathrm{H} & 3.54596 & -1.60039 & -1.67518 \\ \mathrm{H} & 3.86413 & -1.23821 & .05309 \\ \mathrm{H} & 2.42113 & -2.15564 & -.41372\end{array}$

cis-2-butene (B3LYP 6-31G*)

$\mathrm{E}\left(\mathrm{B} 3 \mathrm{LYP} 6-31 \mathrm{G}^{*}\right)=-157.22177$

$\mathrm{E}(\mathrm{B} 3 \mathrm{LYP} 6-31 \mathrm{G} *(\mathrm{THF}))=$ $-157.22324$

$\begin{array}{lccc}\text { C } & .67086 & -.66879 & .00000 \\ \text { C } & .67099 & .66872 & .00000 \\ \text { H } & 1.62634 & 1.19125 & .00000 \\ \text { C } & -.57686 & 1.51718 & .00000 \\ \text { H } & -.33971 & 2.58524 & .00000 \\ \text { H } & -1.20264 & 1.31632 & -.88063 \\ \text { H } & -1.20264 & 1.31632 & .88063 \\ \text { H } & 1.62614 & -1.19138 & .00000 \\ \text { C } & -. .57702 & -1.51713 & .00000 \\ \text { H } & -.34015 & -2.58526 & .00000 \\ \text { H } & -1.20269 & -1.31611 & .88066 \\ \text { H } & -1.20269 & -1.31611 & -.88066\end{array}$

cis-2-butene (B3LYP 631G*(THF))

$\mathrm{E}\left(\mathrm{B} 3 \mathrm{LYP} 6-31 \mathrm{G}^{*}(\mathrm{THF})\right)=$ $-157.22338$ $\begin{array}{llll}\text { C } & .67086 & -.66879 & .00000\end{array}$

$\begin{array}{llll}\text { C } & .67099 & .66872 & .00000\end{array}$

$\begin{array}{llll}\mathrm{H} & 1.62634 & 1.19125 & .00000\end{array}$

$\begin{array}{llll}\text { C } & -.57686 & 1.51718 & .00000\end{array}$

$\begin{array}{llll}\mathrm{H} & -.33971 & 2.58524 & .00000\end{array}$

H $-1.20264 \quad 1.31632-.88063$

H $-1.20264 \quad 1.31632 \quad .88063$

$\begin{array}{llll}\mathrm{H} & 1.62614 & -1.19138 & .00000\end{array}$

C $\quad-.57702-1.51713 \quad .00000$

$\begin{array}{lllll}\mathrm{H} & -.34015 & -2.58526 & .00000\end{array}$

H $-1.20269-1.31611 \quad .88066$

H - $1.20269-1.31611-.88066$

trans-2-butene (B3LYP 6-31G*)

$\mathrm{E}\left(\mathrm{B} 3 \mathrm{LYP} 6-31 \mathrm{G}^{*}\right)=-157.22043$
$\begin{array}{ll}\mathrm{E}(\mathrm{B} 3 \mathrm{LYP} & \left.6-31 \mathrm{G}^{*}(\mathrm{THF})\right)= \\ 157.22201 & =\end{array}$

$\begin{array}{llll}\text { C } & .66699 & -.03128 & .00000\end{array}$

$\begin{array}{llll}\text { C } & -.66699 & .03128 & .00000\end{array}$

H - 1.23279 - $-.90216 \quad .00000$

$\begin{array}{llll}\text { H } & 1.23279 & .90216 & .00000\end{array}$

C $-1.47111 \quad 1.31222 \quad .00000$

$\begin{array}{llll}\mathrm{H} & -2.54640 & 1.10833 & .00000\end{array}$

$\begin{array}{llll}\mathrm{H} & -1.25511 & 1.92724 & .88279\end{array}$

$\begin{array}{llll}\mathrm{H} & -1.25511 & 1.92724 & -.88279\end{array}$

C $1.47111-1.31222 \quad .00000$

$\begin{array}{lllll}\mathrm{H} & 2.54640 & -1.10833 & .00000\end{array}$

$\begin{array}{lllll}\mathrm{H} & 1.25511 & -1.92724 & .88279\end{array}$

$\begin{array}{lllll}\text { H } & 1.25511 & -1.92724 & -.88279\end{array}$

trans-2-butene
31G*(THF)) $\quad$ (B3LYP $\quad$ 6-

$\mathrm{E}\left(\mathrm{B} 3 \mathrm{LYP} 6-31 \mathrm{G}^{*}(\mathrm{THF})\right)=$ $-157.22218$

$\begin{array}{lrrr}\text { C } & .66699 & -.03128 & .00000\end{array}$

$\begin{array}{llll}\text { C } & -.66699 & .03128 & .00000\end{array}$

H $-1.23279-.90216 \quad .00000$

$\begin{array}{llll}\mathrm{H} & 1.23279 & .90216 & .00000\end{array}$

C $-1.47111 \quad 1.31222 \quad .00000$

$\begin{array}{llll}\mathrm{H} & -2.54640 & 1.10833 & .00000\end{array}$

$\begin{array}{llll}\mathrm{H} & -1.25511 & 1.92724 & .88279\end{array}$

$\begin{array}{llll}\mathrm{H} & -1.25511 & 1.92724 & -.88279\end{array}$

$\begin{array}{llll}\text { C } & 1.47111 & -1.31222 & .00000\end{array}$

$\begin{array}{lllll}\mathrm{H} & 2.54640 & -1.10833 & .00000\end{array}$

$\begin{array}{lllll}\mathrm{H} & 1.25511 & -1.92724 & .88279\end{array}$

$\begin{array}{lllll}\text { H } & 1.25511 & -1.92724 & -.88279\end{array}$

\section{C. $\mathrm{Ph}_{3} \mathrm{PCHMe}+$ $\mathrm{MeCHO}$ (reaction 2)}

\section{Ph $_{3}$ PCHMe (B3LYP 6-31G*)}

$\mathrm{E}(\mathrm{B} 3 \mathrm{LYP}$ 6-31G*) $=-1114.8596$

$\mathrm{E}($ B3LYP $6-31 \mathrm{G} *($ THF $))=$ $-1114.86899$

$\begin{array}{cccc}\mathrm{C} & .31793 & -.01981 & .41071 \\ \mathrm{P} & .42453 & -.14559 & 2.08502 \\ \mathrm{H} & -.05943 & .94192 & .06994 \\ \mathrm{C} & 4.33286 & 1.17986 & 4.29616 \\ \mathrm{C} & 3.13247 & 1.16750 & 5.01091 \\ \mathrm{C} & 1.94933 & .76853 & 4.38578 \\ \mathrm{C} & 1.95241 & .37864 & 3.03676 \\ \mathrm{C} & 3.16016 & .40990 & 2.32637 \\ \mathrm{C} & 4.34528 & .80016 & 2.95242 \\ \mathrm{H} & 5.25315 & 1.49113 & 4.78356 \\ \mathrm{H} & 3.11691 & 1.47193 & 6.05457 \\ \mathrm{H} & 1.01966 & .76902 & 4.94979 \\ \mathrm{H} & 3.16438 & .13892 & 1.27329\end{array}$




$\begin{array}{lccc}\text { H } & 5.27562 & .81543 & 2.39036 \\ \mathrm{C} & -.31444 & -4.54481 & 3.39935 \\ \mathrm{C} & .51658 & -3.73749 & 4.17866 \\ \mathrm{C} & .77413 & -2.41958 & 3.79791 \\ \mathrm{C} & .19364 & -1.89181 & 2.63513 \\ \mathrm{C} & -.63864 & -2.71113 & 1.85553 \\ \mathrm{C} & -.88832 & -4.03056 & 2.23464 \\ \mathrm{H} & -.51099 & -5.57159 & 3.69704 \\ \mathrm{H} & .97050 & -4.13496 & 5.08271 \\ \mathrm{H} & 1.43349 & -1.80757 & 4.40435 \\ \mathrm{H} & -1.07574 & -2.29760 & .95084 \\ \mathrm{H} & -1.52983 & -4.65658 & 1.61987 \\ \mathrm{C} & -2.94109 & 2.53996 & 3.79687 \\ \mathrm{C} & -1.71716 & 3.09885 & 3.42488 \\ \mathrm{C} & -.68864 & 2.28171 & 2.95282 \\ \mathrm{C} & -.88389 & .89758 & 2.83123 \\ \mathrm{C} & -2.11667 & .34396 & 3.20282 \\ \mathrm{C} & -3.13769 & 1.16158 & 3.68816 \\ \mathrm{H} & -3.73891 & 3.17619 & 4.17099 \\ \mathrm{H} & -1.55909 & 4.17101 & 3.50672 \\ \mathrm{H} & .26659 & 2.72223 & 2.68151 \\ \mathrm{H} & -2.28025 & -.72541 & 3.10871 \\ \mathrm{H} & -4.08792 & .72165 & 3.97909 \\ \mathrm{C} & 1.06776 & -.87881 & -.58409 \\ \mathrm{H} & 1.32836 & -1.85861 & -.16277 \\ \mathrm{H} & .44978 & -1.07620 & -1.47250 \\ \mathrm{H} & 2.00824 & -.43642 & -.95522\end{array}$

\section{$\mathrm{Ph}_{3}$ PCHMe (B3LYP 31G*(THF))}

\section{$\mathrm{E}(\mathrm{B} 3 \mathrm{LYP} 6-31 \mathrm{G} *(\mathrm{THF}))=$ $-1114.87111$}

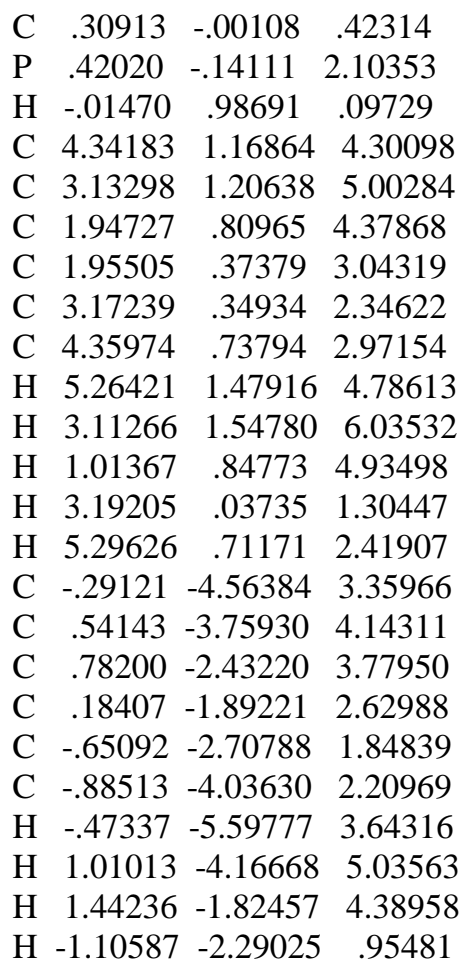

H $-1.52960 \quad-4.65710 \quad 1.59182$

$\begin{array}{llll}\text { C } & -2.96012 & 2.54991 & 3.78768\end{array}$

C -1.757893 .116343 .35590$

$\begin{array}{llll}\text { C } & -.72244 & 2.29913 & 2.89602\end{array}$

$\begin{array}{llll}\text { C } & -.88944 & .90565 & 2.84705\end{array}$

$\begin{array}{llll}\text { C } & -2.09911 & .34385 & 3.28093\end{array}$

$\begin{array}{llll}\text { C } & -3.12727 & 1.16250 & 3.75295\end{array}$

H $-3.76345 \quad 3.18680 \quad 4.15024$

H $-1.62193 \quad 4.19495 \quad 3.38032$

H $\quad .21249 \quad 2.75132 \quad 2.57568$

H $-2.24299 \quad-.73220 \quad 3.24507$

$\begin{array}{llll}\text { H } & -4.05965 & .71619 & 4.09038\end{array}$

$\begin{array}{llll}\text { C } & 1.07558 & -.85135 & -.56833\end{array}$

H $1.26044 \quad-1.85998 \quad-.17677$

H $\quad .50304 \quad-.98316-1.49859$

$\begin{array}{lllll}\text { H } & 2.05855 & -.44943 & -.87070\end{array}$

\section{Cis TS1 (B3LYP 6-31G*)}

E(B3LYP 6-31G*) $=-1268.68607$

$\mathrm{E}(\mathrm{B} 3 \mathrm{LYP} 6-31 \mathrm{G} *(\mathrm{THF}))=$ $-1268.70809$

$\begin{array}{lccl}\mathrm{O} & 1.92894 & .30086 & 2.18194 \\ \mathrm{C} & 1.74938 & 1.01717 & 1.15010 \\ \mathrm{P} & -1.02504 & .21610 & -.09168 \\ \mathrm{C} & .72470 & -.03320 & -.19603 \\ \mathrm{C} & -1.97025 & -.65223 & -1.40652\end{array}$

6- $\quad$ C $-2.20494-2.03528-1.29765$

$\begin{array}{lll}\text { C }-2.76054 & -2.74106 & -2.36402\end{array}$

$\begin{array}{llll}\text { C } & -3.08611 & -2.08040 & -3.55085\end{array}$

$\begin{array}{llll}\text { C } & -2.85454 & -.70831 & -3.66717\end{array}$

$\begin{array}{llll}\text { C } & -2.29777 & .00288 & -2.60413\end{array}$

$\begin{array}{llll}\text { C } & -1.37993 & 2.00089 & -.28996\end{array}$

$\begin{array}{llll}\text { C } & -1.75196 & -.32566 & 1.48979\end{array}$

$\begin{array}{llll}\text { C } & -.51540 & 2.81393 & -1.04004\end{array}$

$\begin{array}{llll}\text { C } & -.78090 & 4.17519 & -1.18602\end{array}$

C $-1.90708 \quad 4.73895 \quad-.58371$

$\begin{array}{llll}\text { C } & -2.76954 & 3.93635 & .16461\end{array}$

$\begin{array}{llll}\text { C } & -2.50903 & 2.57382 & .31277\end{array}$

$\begin{array}{llll}\text { C } & -.96566 & -.32767 & 2.65443\end{array}$

$\begin{array}{llll}\text { C } & -1.55687 & -.68192 & 3.87048\end{array}$

$\begin{array}{llll}\text { C } & -2.90782 & -1.02378 & 3.93844\end{array}$

$\begin{array}{llll}\text { C } & -3.68820 & -1.01699 & 2.77930\end{array}$

$\begin{array}{llll}\text { C } & -3.11477 & -.67103 & 1.55689\end{array}$

$\begin{array}{llll}\mathrm{H} & 1.06081 & 1.89092 & 1.21780\end{array}$

H $1.02906 \quad 35805 \quad-1.17169$

H $-1.96524 \quad-2.55810 \quad-.37661$

H $-2.93971-3.80825-2.26572$

H $-3.51753-2.63290 \quad-4.38085$

H $-3.10953-.18816 \quad-4.58640$

$\begin{array}{llll}\mathrm{H} & -2.12011 & 1.06930 & -2.70684\end{array}$

H $\quad .37453 \quad 2.39461 \quad-1.49631$

H $\quad-.09886 \quad 4.79536 \quad-1.76069$

H $-2.10663 \quad 5.80153-.69072$

$\begin{array}{llll}\text { H } & -3.64211 & 4.37092 & .64417\end{array}$

$\begin{array}{llll}\mathrm{H} & -3.17576 & 1.96341 & .91380\end{array}$

H $\quad .10307 \quad-.07275 \quad 2.61741$

\begin{abstract}
$\begin{array}{llll}\mathrm{H} & -.94641 & -.69119 & 4.76945\end{array}$
H $-3.35464-1.29897 \quad 4.89057$

H $-4.74042-1.28487 \quad 2.82426$

$\begin{array}{llll}\text { H } & -3.72609 & -.68001 & .65919\end{array}$

$\begin{array}{llll}\text { C } & 2.98810 & 1.36205 & .30914\end{array}$

$\begin{array}{llll}\mathrm{H} & 2.74866 & 1.93539 & -.59562\end{array}$

$\begin{array}{llll}\text { H } & 3.66256 & 1.96460 & .93114\end{array}$

$\begin{array}{llll}\mathrm{H} & 3.52733 & .45036 & .02944\end{array}$

$\begin{array}{llll}\text { C } & 1.19266 & -1.46775 & .03280\end{array}$

H $\quad .51655 \quad-2.24974 \quad-.33795$

H $2.15576-1.61536-.46696$

H 1.36685 -1.60956 1.10710
\end{abstract}

\section{Cis TS1b (B3L YP 6-31G*)}

$\mathrm{E}\left(\mathrm{B} 3 \mathrm{LYP} 6-31 \mathrm{G}^{*}\right)=-1268.68204$ $\mathrm{E}\left(\right.$ B3LYP $\left.6-31 \mathrm{G}^{*}(\mathrm{THF})\right)=$ $-1268.70119$

$\begin{array}{lccc}\text { O } & 1.01129 & 1.58766 & 2.06117 \\ \text { C } & 1.65773 & 1.26852 & 1.02358 \\ \text { P } & -.99078 & .14828 & -.06339 \\ \text { C } & .74948 & -.20703 & -.07418 \\ \text { C } & -1.93259 & -.64144 & -1.43535 \\ \text { C } & -1.94183 & -2.04672 & -1.51890 \\ \text { C } & -2.57195 & -2.68693 & -2.58360 \\ \text { C } & -3.19711 & -1.93662 & -3.58382 \\ \text { C } & -3.19338 & -.54376 & -3.50845 \\ \text { C } & -2.56663 & .10278 & -2.44084 \\ \text { C } & -1.26484 & 1.94282 & -.25460 \\ \text { C } & -1.80341 & -.41196 & 1.46907 \\ \text { C } & -.60397 & 2.64237 & -1.27697 \\ \text { C } & -.81876 & 4.01091 & -1.44093 \\ \text { C } & -1.69071 & 4.69035 & -.58823 \\ \text { C } & -2.34769 & 3.99877 & .43042 \\ \text { C } & -2.13639 & 2.63057 & .59941 \\ \text { C } & -1.14078 & -.23858 & 2.69734 \\ \text { C } & -1.77556 & -.63579 & 3.87603 \\ \text { C } & -3.05349 & -1.19673 & 3.84408 \\ \text { C } & -3.71490 & -1.35573 & 2.62421 \\ \text { C } & -3.09561 & -.96402 & 1.43772 \\ \text { H } & 1.62559 & 1.96189 & .15088 \\ \text { H } & 1.11882 & .03302 & -1.07912 \\ \text { H } & -1.46844 & -2.64177 & -.74374 \\ \text { H } & -2.57474 & -3.77239 & -2.63248 \\ \text { H } & -3.68607 & -2.43718 & -4.41500 \\ \text { H } & -3.68309 & .04589 & -4.27875 \\ \text { H } & -2.57632 & 1.18668 & -2.39204 \\ \text { H } & .07863 & 2.12610 & -1.94559 \\ \text { H } & -.29917 & 4.54559 & -2.23111 \\ \text { H } & -1.85373 & 5.75714 & -.71506 \\ \text { H } & -3.02022 & 4.52461 & 1.10227 \\ \text { H } & -2.63829 & 2.10419 & 1.40444 \\ \text { H } & -.16213 & .24957 & 2.71606 \\ \text { H } & -1.26411 & -.49975 & 4.82518 \\ \text { H } & -3.53691 & -1.50614 & 4.76736 \\ \text { H } & -4.71310 & -1.78409 & 2.59340 \\ \text { H } & -3.61964 & -1.09003 & .49583\end{array}$




$\begin{array}{cccc}\mathrm{C} & 3.05355 & .65890 & 1.17868 \\ \mathrm{H} & 3.47868 & .31261 & .22842 \\ \mathrm{H} & 3.71232 & 1.43810 & 1.58531 \\ \mathrm{H} & 3.04011 & -.16718 & 1.89715 \\ \mathrm{C} & 1.12406 & -1.63292 & .32801 \\ \mathrm{H} & .79264 & -2.40708 & -.38135 \\ \mathrm{H} & 2.21426 & -1.71360 & .39015 \\ \mathrm{H} & .72736 & -1.89503 & 1.31505\end{array}$

\section{Cis TS1 (B3LYP 6-31G*(THF))}

E $($ B3LYP 6-31G*(THF) $)=$
-1268.70937

$\begin{array}{llll}\text { O } & 1.85578 & 1.14200 & 2.96608\end{array}$

$\begin{array}{lrrr}\text { C } & 2.04010 & 1.55600 & 1.83017\end{array}$

$\begin{array}{llll}\mathrm{P} & -1.07435 & .06750 & -.13965\end{array}$

$\begin{array}{llll}\text { C } & .59151 & -.25621 & .00803\end{array}$

C $-2.08933-.70003 \quad-1.50529$

C $-1.85763-2.05711-1.79381$

$\begin{array}{llll}\text { C }-2.52148 & -2.68378 & -2.84876\end{array}$

C $-3.42035-1.95922-3.63980$

$\begin{array}{llll}\text { C } & -3.65624 & -.61030 & -3.36396\end{array}$

$\begin{array}{llll}\text { C } & -2.99568 & .01613 & -2.30174\end{array}$

$\begin{array}{llll}\text { C } & -1.25255 & 1.87179 & -.39643\end{array}$

$\begin{array}{llll}\text { C } & -1.98185 & -.42527 & 1.37872\end{array}$

$\begin{array}{llll}\text { C } & -.67997 & 2.46899 & -1.53328\end{array}$

C $-.72022 \quad 3.85473-1.69573$

C $-1.34147 \quad 4.65797 \quad-.73396$

$\begin{array}{llll}\text { C } & -1.92219 & 4.06919 & .39177\end{array}$

$\begin{array}{llll}\mathrm{C} & -1.87828 & 2.68283 & .56161\end{array}$

C $-1.33362-.30646 \quad 2.62011$

$\begin{array}{llll}\text { C } & -2.00339 & -.65596 & 3.79526\end{array}$

C $-3.32157-1.11834 \quad 3.74496$

$\begin{array}{llll}\text { C } & -3.97346 & -1.22973 & 2.51347\end{array}$

C $-3.30733-.88696 \quad 1.33511$

$\begin{array}{llll}\mathrm{H} & 1.29711 & 2.20881 & 1.33920\end{array}$

$\begin{array}{lllll}\mathrm{H} & 1.17477 & .31481 & -.71497\end{array}$

H $-1.14965-2.62686-1.19597$

H $-2.33208-3.73374-3.05958$

H $-3.93042-2.44410-4.46876$

H $-4.35303-.04224 \quad-3.97618$

H $-3.18823 \quad 1.06572-2.09723$

$\mathrm{H} \quad-.20098 \quad 1.85602 \quad-2.29286$

$\begin{array}{llll}\mathrm{H} & -.26945 & 4.30621 & -2.57628\end{array}$

H $-1.37278 \quad 5.73717 \quad-.86285$

H $-2.40910 \quad 4.68732 \quad 1.14231$

H $-2.325392 .23797 \quad 1.44566$

$\begin{array}{llll}\mathrm{H} & -.31303 & .06323 & 2.66226\end{array}$

H $-1.49401 \quad-.56649 \quad 4.75174$

H $-3.83947-1.39027 \quad 4.66184$

H $-4.99902-1.588302 .46808$

$\begin{array}{llll}\mathrm{H} & -3.82098 & -.98500 & .38361\end{array}$

$\begin{array}{lrrr}\text { C } & 3.32998 & 1.34060 & 1.07979\end{array}$

$\begin{array}{llll}\text { H } & 3.15808 & 1.24957 & .00427\end{array}$

$\begin{array}{llll}\mathrm{H} & 3.97341 & 2.21908 & 1.24009\end{array}$

$\begin{array}{llll}\mathrm{H} & 3.85171 & .45810 & 1.46172\end{array}$

$\begin{array}{llll}\text { C } & 1.14691 & -1.60846 & .40858\end{array}$

H $1.27993 \quad-2.32109-.42329$

$\begin{array}{llll}\mathrm{H} & 2.13239 & -1.49315 & .88337\end{array}$

H $\quad .50582-2.10385 \quad 1.14889$

\section{Cis TS1b (B3L YP 6-31G*(THF))} E $($ B3LYP 6-31G*(THF) $)=$
-1268.70763

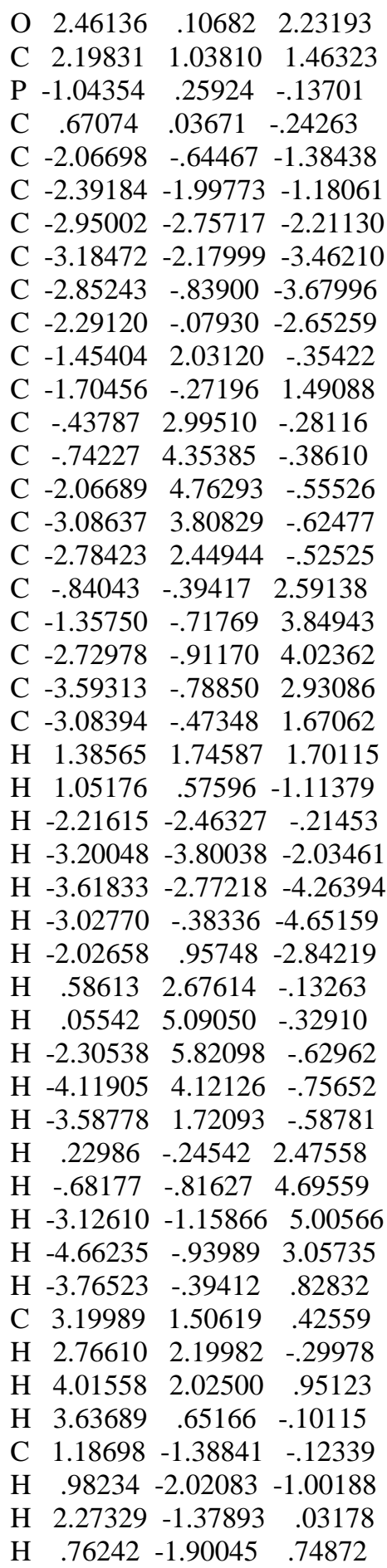

Trans TS1 (B3LYP 6-31G*)
$\mathrm{E}\left(\mathrm{B} 3 \mathrm{LYP} 6-31 \mathrm{G}^{*}\right)=-1268.68678$ $\mathrm{E}\left(\mathrm{B} 3 \mathrm{LYP} 6-31 \mathrm{G}^{*}(\mathrm{THF})\right)=$ $-1268.70279$

$\begin{array}{llll}\text { O } & 1.76394 & 1.51110 & 1.61454\end{array}$

$\begin{array}{llll}\text { C } & 2.10954 & .52683 & .90665\end{array}$

$\begin{array}{llll}\mathrm{P} & -1.04510 & .27248 & -.17309\end{array}$

$\begin{array}{llll}\text { C } & .64818 & -.16136 & -.44595\end{array}$

$\begin{array}{llll}\text { C } & -2.13804 & -.60787 & -1.36486\end{array}$

C $-2.47998-1.95477-1.14466$

C $-3.11050 \quad-2.70089-2.14152$

C $-3.40246-2.11968-3.37610$

$\begin{array}{llll}\text { C } & -3.04864 & -.78960 & -3.61314\end{array}$

$\begin{array}{llll}\text { C } & -2.41453 & -.04283 & -2.62209\end{array}$

$\begin{array}{llll}\text { C } & -1.32414 & 2.06427 & -.37897\end{array}$

$\begin{array}{llll}\text { C } & -1.62401 & -.15259 & 1.51130\end{array}$

$\begin{array}{llll}\text { C } & -.29564 & 2.96478 & -.05643\end{array}$

$\begin{array}{llll}\text { C } & -.54176 & 4.33846 & -.12599\end{array}$

$\begin{array}{llll}\text { C } & -1.79300 & 4.82019 & -.50752\end{array}$

$\begin{array}{llll}\text { C } & -2.82231 & 3.92410 & -.80620\end{array}$

$\begin{array}{llll}\text { C } & -2.59430 & 2.55133 & -.73555\end{array}$

$\begin{array}{llll}\text { C } & -.76143 & .08582 & 2.59403\end{array}$

$\begin{array}{llll}\text { C } & -1.21684 & -.13750 & 3.89504\end{array}$

$\begin{array}{llll}\text { C } & -2.51916 & -.58228 & 4.12943\end{array}$

$\begin{array}{llll}\text { C } & -3.38566 & -.79081 & 3.05449\end{array}$

$\begin{array}{llll}\text { C } & -2.94393 & -.57054 & 1.75000\end{array}$

$\begin{array}{llll}\mathrm{H} & 2.13741 & -.48285 & 1.37188\end{array}$

H $\quad .94995 \quad .34355 \quad-1.36949$

H $-2.25552-2.42620-.19311$

H $-3.36957-3.73878-1.95022$

H $-3.89301-2.70132-4.15159$

$\begin{array}{llll}\mathrm{H} & -3.26361 & -.33072 & -4.57423\end{array}$

$\begin{array}{llll}\mathrm{H} & -2.13361 & .98488 & -2.82985\end{array}$

$\begin{array}{lllll}\mathrm{H} & .66355 & 2.60374 & .31760\end{array}$

$\begin{array}{llll}\mathrm{H} & .25362 & 5.03210 & .13277\end{array}$

$\begin{array}{llll}\mathrm{H} & -1.97315 & 5.89079 & -.56073\end{array}$

H $-3.80514 \quad 4.29270-1.08738$

$\begin{array}{llll}\mathrm{H} & -3.40583 & 1.86472 & -.95730\end{array}$

$\begin{array}{llll}\mathrm{H} & .23441 & .49878 & 2.42722\end{array}$

$\begin{array}{llll}\mathrm{H} & -.54618 & .04895 & 4.72950\end{array}$

H $-2.86343-.75467 \quad 5.14612$

H $-4.40613 \quad-1.12157 \quad 3.22870$

$\begin{array}{llll}\mathrm{H} & -3.63062 & -.72450 & .92353\end{array}$

$\begin{array}{llll}\text { C } & 3.21474 & .73337 & -.13004\end{array}$

$\begin{array}{llll}\text { H } & 3.36042 & -.12970 & -.78907\end{array}$

$\begin{array}{llll}\mathrm{H} & 3.00847 & 1.62353 & -.73402\end{array}$

$\begin{array}{llll}\mathrm{H} & 4.15415 & .91121 & .41057\end{array}$

C $\quad .89865-1.66591-.49203$

$\begin{array}{llll}\mathrm{H} & 1.97705 & -1.85106 & -.57499\end{array}$

$\begin{array}{llll}\text { H } & .56977 & -2.15282 & .43568\end{array}$

H $\quad .41494 \quad-2.18871-1.33046$

Trans TS1
31G*(THF $)$

$\mathrm{E}\left(\mathrm{B} 3 \mathrm{LYP} 6-31 \mathrm{G}^{*}(\mathrm{THF})\right)=$ $-1268.70695$ 


$\begin{array}{llll}\text { O } & 2.06454 & 1.60182 & 1.82953 \\ \text { C } & 2.41406 & .65906 & 1.10650 \\ \text { P } & -1.06240 & .25782 & -.18436 \\ \text { C } & .61790 & -.13402 & -.39346 \\ \text { C } & -2.17408 & -.59456 & -1.38800 \\ \text { C } & -2.49166 & -1.95206 & -1.19208 \\ \text { C } & -3.10664 & -2.69352 & -2.20323 \\ \text { C } & -3.40493 & -2.09691 & -3.43080 \\ \text { C } & -3.07566 & -.75471 & -3.64472 \\ \text { C } & -2.45816 & -.01241 & -2.63712 \\ \text { C } & -1.33138 & 2.05492 & -.36294 \\ \text { C } & -1.66778 & -.18622 & 1.48961 \\ \text { C } & -.26913 & 2.93741 & -.11064 \\ \text { C } & -.48127 & 4.31737 & -.17597 \\ \text { C } & -1.74750 & 4.82487 & -.47670 \\ \text { C } & -2.81338 & 3.94808 & -.70200 \\ \text { C } & -2.60956 & 2.56931 & -.64077 \\ \text { C } & -.80140 & .01355 & 2.57793 \\ \text { C } & -1.25117 & -.22543 & 3.87887 \\ \text { C } & -2.56218 & -.65275 & 4.10730 \\ \text { C } & -3.43299 & -.82973 & 3.02871 \\ \text { C } & -2.99081 & -.59361 & 1.72522 \\ \text { H } & 2.32429 & -.38144 & 1.46648 \\ \text { H } & .98318 & .37973 & -1.28752 \\ \text { H } & -2.26345 & -2.43768 & -.24733 \\ \text { H } & -3.34949 & -3.73882 & -2.02834 \\ \text { H } & -3.88199 & -2.67471 & -4.21833 \\ \text { H } & -3.29666 & -.28311 & -4.59926 \\ \text { H } & -2.19510 & 1.02365 & -2.82971 \\ \text { H } & .70444 & 2.55121 & .17399 \\ \text { H } & .34505 & 4.99643 & .01936 \\ \text { H } & -1.90737 & 5.89942 & -.52448 \\ \text { H } & -3.80451 & 4.33632 & -.92304 \\ \text { H } & -3.44764 & 1.89929 & -.81166 \\ \text { H } & .20423 & .39210 & 2.41480 \\ \text { H } & -.57625 & -.06771 & 4.71669 \\ \text { H } & -2.90695 & -.83683 & 5.12209 \\ \text { H } & -4.45785 & -1.15036 & 3.19914 \\ \text { H } & -3.67961 & -.72999 & .89738 \\ \text { C } & 3.38586 & .86371 & -.03962 \\ \text { H } & 3.37436 & .03584 & -.75283 \\ \text { H } & 3.18067 & 1.80557 & -.55785 \\ \text { H } & 4.39932 & .92920 & .38543 \\ \text { C } & .94369 & -1.62163 & -.36031 \\ \text { H } & 2.02954 & -1.77164 & -.42837 \\ \text { H } & .62632 & -2.07963 & .58659 \\ \text { H } & .48992 & -2.21648 & -1.17073\end{array}$

TSbetaine (B3LYP 6-31G*(THF))

$\mathrm{E}(\mathrm{B} 3 \mathrm{LYP} 6-31 \mathrm{G} *(\mathrm{THF}))=$ $-1268.70453$

$\begin{array}{lrcl}\mathrm{O} & 3.17829 & .87162 & .84886 \\ \mathrm{C} & 2.05950 & 1.04753 & 1.38716 \\ \mathrm{P} & -1.03291 & .28511 & -.07873 \\ \mathrm{C} & .71703 & .09841 & -.02706\end{array}$

$\begin{array}{llll}\text { C } & -1.93291 & -.66152 & -1.38329\end{array}$

C $-1.89222-2.06802-1.32728$

C $-2.45840-2.83143-2.34780$

C $-3.07161-2.20355-3.43705$

$\begin{array}{llll}\text { C } & -3.11753 & -.80890 & -3.49920\end{array}$

$\begin{array}{llll}\text { C } & -2.54888 & -.03952 & -2.48013\end{array}$

$\begin{array}{llll}\text { C } & -1.39701 & 2.05373 & -.35109\end{array}$

$\begin{array}{llll}\text { C } & -1.81037 & -.26475 & 1.48055\end{array}$

$\begin{array}{llll}\text { C } & -.76782 & 2.72497 & -1.41533\end{array}$

$\begin{array}{llll}\text { C } & -.98788 & 4.08854 & -1.61233\end{array}$

C $-1.83178 \quad 4.79533 \quad-.75008$

$\begin{array}{llll}\text { C } & -2.45966 & 4.13309 & .30711\end{array}$

$\begin{array}{llll}\text { C } & -2.24680 & 2.76656 & .50678\end{array}$

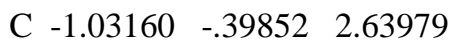

C $-1.61875 \quad-.804693 .83962$

$\begin{array}{llll}\text { C } & -2.98887 & -1.06975 & 3.89374\end{array}$

$\begin{array}{llll}\text { C } & -3.77265 & -.93264 & 2.74331\end{array}$

C $-3.18796-.53776 \quad 1.53923$

$\begin{array}{llll}\mathrm{H} & 1.75732 & .36721 & 2.21143\end{array}$

$\begin{array}{lllll}\mathrm{H} & 1.12969 & .68469 & -.85247\end{array}$

H $-1.42514-2.57128-.48482$

H $-2.42028-3.91648-2.29215$

H $-3.51155-2.80012-4.23231$

H $-3.59777-.31518$ - 4.34045

H $-2.59184 \quad 1.04338-2.54261$

$\begin{array}{llll}\mathrm{H} & -.10310 & 2.19290 & -2.09078\end{array}$

H $\quad-.49569 \quad 4.59732 \quad-2.43720$

H $-1.99696 \quad 5.85904 \quad-.90165$

$\begin{array}{llll}\mathrm{H} & -3.11397 & 4.67871 & .98218\end{array}$

H $-2.734792 .26595 \quad 1.33737$

$\begin{array}{llll}\mathrm{H} & .03113 & -.19217 & 2.60055\end{array}$

H $-1.00308-.91389 \quad 4.72899$

H $-3.44534-1.38501 \quad 4.82867$

H $-4.83894-1.139852 .78168$

$\begin{array}{llll}\text { H } & -3.80269 & -.44798 & .64769\end{array}$

$\begin{array}{llll}\text { C } & 1.49625 & 2.45423 & 1.53036\end{array}$

H $\quad .47338 \quad 2.46058 \quad 1.91773$

$\begin{array}{llll}\text { H } & 2.13659 & 3.00721 & 2.23388\end{array}$

$\begin{array}{llll}\mathrm{H} & 1.52029 & 2.98403 & .57331\end{array}$

$\begin{array}{llll}\text { C } & 1.24565 & -1.33144 & -.01499\end{array}$

$\begin{array}{llll}\mathrm{H} & 1.06613 & -1.89249 & -.94388\end{array}$

H $2.32939 \quad-1.27844 \quad .14235$

H $\quad .81956 \quad-1.92050 \quad .80807$

\section{Betaine (B3LYP 6-31G*(THF))}

$\mathrm{E}(\mathrm{B} 3 \mathrm{LYP}$ 6-31G*(THF) $)=$ $-1268.71208$

$\begin{array}{llll}\mathrm{O} & 3.00400 & .70204 & .43196 \\ \mathrm{C} & 1.78885 & .78478 & .95092 \\ \mathrm{P} & -1.06746 & .31059 & -.05053 \\ \mathrm{C} & .76516 & .08588 & -.12363 \\ \mathrm{C} & -1.88916 & -.66194 & -1.36855 \\ \mathrm{C} & -2.00293 & -2.05653 & -1.21193 \\ \mathrm{C} & -2.55047 & -2.83423 & -2.23127 \\ \mathrm{C} & -2.98908 & -2.23338 & -3.41574 \\ \mathrm{C} & -2.88190 & -.85007 & -3.57509\end{array}$

$\begin{array}{cccc}\text { C } & -2.33481 & -.06419 & -2.55784 \\ \text { C } & -1.46436 & 2.06949 & -.32814 \\ \text { C } & -1.81406 & -.24482 & 1.51544 \\ \text { C } & -.83554 & 2.75769 & -1.38187 \\ \text { C } & -1.11842 & 4.10547 & -1.60367 \\ \text { C } & -2.02139 & 4.77903 & -.77563 \\ \text { C } & -2.64595 & 4.09948 & .27271 \\ \text { C } & -2.37315 & 2.74808 & .49703 \\ \text { C } & -1.03899 & -.39222 & 2.67659 \\ \text { C } & -1.63759 & -.79747 & 3.87100 \\ \text { C } & -3.00965 & -1.05123 & 3.91896 \\ \text { C } & -3.78797 & -.90262 & 2.76628 \\ \text { C } & -3.19642 & -.50530 & 1.56735 \\ \text { H } & 1.64667 & .16478 & 1.88285 \\ \text { H } & 1.02514 & .58505 & -1.06702 \\ \text { H } & -1.67160 & -2.53825 & -.29650 \\ \text { H } & -2.63399 & -3.90979 & -2.09870 \\ \text { H } & -3.41488 & -2.84213 & -4.20927 \\ \text { H } & -3.22751 & -.37642 & -4.49035 \\ \text { H } & -2.26612 & 1.00976 & -2.69604 \\ \text { H } & -.11930 & 2.25779 & -2.02782 \\ \text { H } & -.62544 & 4.62830 & -2.41894 \\ \text { H } & -2.23364 & 5.83142 & -.94570 \\ \text { H } & -3.34459 & 4.61975 & .92265 \\ \text { H } & -2.85928 & 2.23652 & 1.32167 \\ \text { H } & .02836 & -.20129 & 2.65289 \\ \text { H } & -1.02508 & -.91646 & 4.76110 \\ \text { H } & -3.47233 & -1.36695 & 4.85056 \\ \text { H } & -4.85600 & -1.10082 & 2.79823 \\ \text { H } & -3.81090 & -.40439 & .67705 \\ \text { C } & 1.36998 & 2.22422 & 1.32662 \\ \text { H } & .38432 & 2.30050 & 1.80596 \\ \text { H } & 2.11857 & 2.61371 & 2.02659 \\ \text { H } & 1.37622 & 2.87093 & .44185 \\ \text { C } & 1.13482 & -1.40162 & -.23293 \\ \text { H } & .76667 & -1.88008 & -1.14514 \\ \text { H } & 2.22826 & -1.41808 & -.23330 \\ \text { H } & .77981 & -1.97704 & .63106\end{array}$

Cis OP1 (B3LYP 6-31G*)

$\mathrm{E}\left(\mathrm{B} 3 \mathrm{LYP} 6-31 \mathrm{G}^{*}\right)=-1268.72326$ $\mathrm{E}($ B3LYP $6-31 \mathrm{G} *(\mathrm{THF}))=$ $-1268.73477$

$\begin{array}{llll}\mathrm{O} & .16758 & .50226 & -.02498 \\ \mathrm{C} & .20753 & .14805 & 1.34896 \\ \mathrm{C} & 1.72044 & .35659 & 1.58580 \\ \mathrm{P} & 1.97339 & .32410 & -.29986 \\ \mathrm{H} & -.04040 & -.92001 & 1.48206 \\ \mathrm{H} & 2.23636 & -.48850 & 2.06078 \\ \mathrm{C} & 1.76540 & 3.59491 & -3.63673 \\ \mathrm{C} & .81267 & 3.57707 & -2.61831 \\ \mathrm{C} & .86712 & 2.60450 & -1.61728 \\ \mathrm{C} & 1.89754 & 1.65249 & -1.60437 \\ \mathrm{C} & 2.85794 & 1.68554 & -2.63135 \\ \mathrm{C} & 2.78282 & 2.63790 & -3.64695 \\ \mathrm{H} & 1.71530 & 4.34647 & -4.42053\end{array}$




\begin{abstract}
H $\quad .01455 \quad 4.31481 \quad-2.60489$
$\mathrm{H} \quad .10303 \quad 2.55702 \quad-.85253$

H $3.66658 \quad .96223-2.64118$

$\begin{array}{llll}\text { H } 3.52526 & 2.63654 & -4.44068\end{array}$

$\begin{array}{llll}\text { C } 6.71406 & .40172 & .18436\end{array}$

$\begin{array}{lll}\text { C } 5.96087 & 1.57748 & .22082\end{array}$

$\begin{array}{llll}\text { C } 4.58058 & 1.53011 & .02429\end{array}$

$\begin{array}{llll}\text { C } 3.90676 & .31569 & -.20807\end{array}$

$\begin{array}{llll}\text { C } 4.68411 & -.85173 & -.24219\end{array}$

$\begin{array}{llll}\text { C } 6.06953 & -.81154 & -.05077\end{array}$

$\begin{array}{llll}\text { H } 7.79046 & .43391 & .33211\end{array}$

$\begin{array}{llll}\text { H } & 6.44871 & 2.53371 & .39488\end{array}$

$\begin{array}{llll}\text { H } & 4.02348 & 2.46429 & .03129\end{array}$

H $4.21649-1.81250 \quad-.43241$

H $6.64332-1.73448-.09268$

$\begin{array}{llll}\text { C } & 1.25893 & -3.89870 & -2.12332\end{array}$

$\begin{array}{llll}\text { C } & 1.22815 & -2.76455 & -2.93614\end{array}$

$\begin{array}{llll}\text { C } & 1.46144 & -1.50030 & -2.39394\end{array}$

$\begin{array}{llll}\text { C } & 1.73730 & -1.35161 & -1.02742\end{array}$

$\begin{array}{llll}\text { C } & 1.76373 & -2.49623 & -.21607\end{array}$

$\begin{array}{llll}\text { C } & 1.52539 & -3.75965 & -.76092\end{array}$

H $1.07501-4.88215 \quad-2.54743$

$\begin{array}{llll}\text { H } & 1.01736 & -2.86010 & -3.99818\end{array}$

H $1.42042 \quad-.62856 \quad-3.03904$

H $1.97739-2.41356 \quad .84585$

H $1.55005 \quad-4.63449 \quad-.11639$

C $-\begin{array}{llll}\text { C.77713 } & .97007 & 2.17312\end{array}$

H $-.73595 \quad .69275 \quad 3.23392$

H -.576912 .042592 .08472$

H - $1.79405 \quad .78532 \quad 1.81142$

C $2.13449 \quad 1.649642 .29222$

H $3.22215 \quad 1.760592 .30298$

H $1.70600 \quad 2.53661 \quad 1.81262$

H $1.78746 \quad 1.63448 \quad 3.33256$
\end{abstract}

\section{Cis OP1 (B3LYP 6-31G*(THF))}

$\mathrm{E}(\mathrm{B} 3 \mathrm{LYP} 6-31 \mathrm{G} *(\mathrm{THF}))=$
-1268.73786

$\begin{array}{cccc}\mathrm{O} & .13889 & .47340 & -.03796 \\ \mathrm{C} & .19684 & .14195 & 1.34399 \\ \mathrm{C} & 1.70705 & .37513 & 1.56860 \\ \mathrm{P} & 1.97247 & .31162 & -.31006 \\ \mathrm{H} & -.03075 & -.92793 & 1.49662 \\ \mathrm{H} & 2.23115 & -.45368 & 2.06235 \\ \mathrm{C} & 1.77713 & 3.64278 & -3.58751 \\ \mathrm{C} & .87118 & 3.65799 & -2.52540 \\ \mathrm{C} & .91586 & 2.66126 & -1.54600 \\ \mathrm{C} & 1.89010 & 1.65284 & -1.59795 \\ \mathrm{C} & 2.80905 & 1.65765 & -2.66334 \\ \mathrm{C} & 2.74075 & 2.63212 & -3.65969 \\ \mathrm{H} & 1.73323 & 4.41120 & -4.35587 \\ \mathrm{H} & .11785 & 4.43973 & -2.46097 \\ \mathrm{H} & .18466 & 2.64800 & -.74745 \\ \mathrm{H} & 3.58072 & .89597 & -2.71970 \\ \mathrm{H} & 3.44695 & 2.60718 & -4.48618 \\ \mathrm{C} & 6.71299 & .40677 & .17831\end{array}$

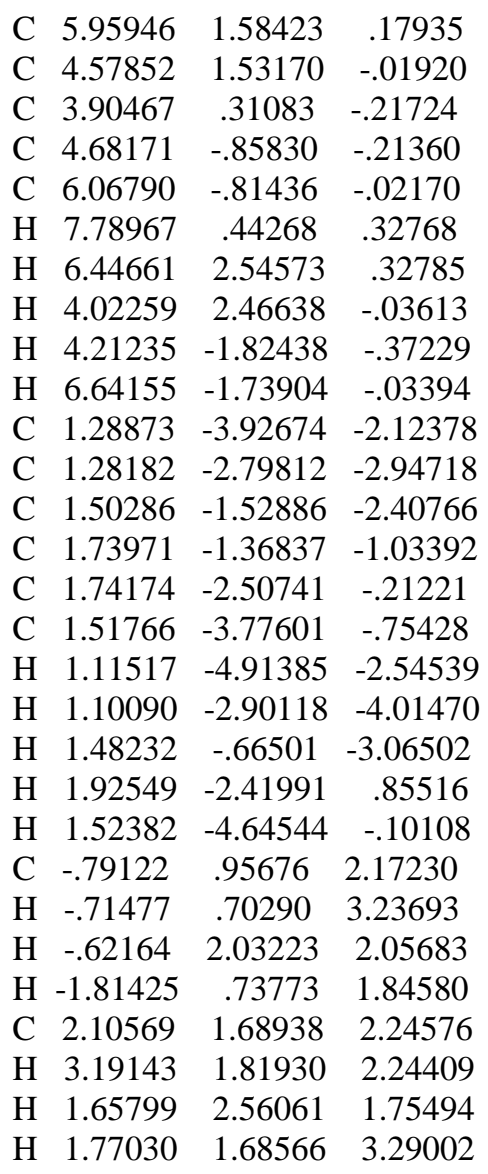

\section{Trans OP1 (B3LYP 6-31G*)}

E(B3LYP 6-31G*) $=-1268.72667$

$\mathrm{E}\left(\mathrm{B} 3 \mathrm{LYP} 6-31 \mathrm{G}^{*}(\mathrm{THF})\right)=$ $-1268.73699$

$\begin{array}{lccc}\mathrm{O} & 1.20221 & .11113 & 3.67578 \\ \mathrm{C} & .44576 & -.90032 & 3.02558 \\ \mathrm{C} & -.39274 & -.00685 & 2.10703 \\ \mathrm{P} & .68591 & 1.49917 & 2.60068 \\ \mathrm{H} & -1.38923 & .17488 & 2.53478 \\ \mathrm{C} & 4.94139 & 1.93988 & .71100 \\ \mathrm{C} & 4.56704 & .76133 & 1.35597 \\ \mathrm{C} & 3.29186 & .63413 & 1.90885 \\ \mathrm{C} & 2.35403 & 1.67387 & 1.80071 \\ \mathrm{C} & 2.74729 & 2.85832 & 1.15461 \\ \mathrm{C} & 4.02951 & 2.99268 & .62217 \\ \mathrm{H} & 5.93784 & 2.04178 & .28904 \\ \mathrm{H} & 5.27257 & -.06063 & 1.44494 \\ \mathrm{H} & 3.02720 & -.25787 & 2.46139 \\ \mathrm{H} & 2.05306 & 3.68515 & 1.05940 \\ \mathrm{H} & 4.31242 & 3.92236 & .13554 \\ \mathrm{C} & -2.17408 & 4.09471 & -.18108 \\ \mathrm{C} & -1.05461 & 3.47302 & -.73498 \\ \mathrm{C} & -.15960 & 2.77656 & .08176 \\ \mathrm{C} & -.34087 & 2.69483 & 1.47418 \\ \mathrm{C} & -1.48835 & 3.31141 & 2.00412 \\ \mathrm{C} & -2.39149 & 4.00333 & 1.19411\end{array}$

$\begin{array}{cccc}\mathrm{H} & -2.87092 & 4.63811 & -.81383 \\ \mathrm{H} & -.87467 & 3.52631 & -1.80612 \\ \mathrm{H} & .69421 & 2.29210 & -.38378 \\ \mathrm{H} & -1.68282 & 3.26257 & 3.07255 \\ \mathrm{H} & -3.26355 & 4.47439 & 1.64139 \\ \mathrm{C} & .66556 & 4.30646 & 6.35440 \\ \mathrm{C} & .90543 & 4.82427 & 5.07974 \\ \mathrm{C} & .87166 & 3.98616 & 3.96656 \\ \mathrm{C} & .62856 & 2.60976 & 4.10685 \\ \mathrm{C} & .40714 & 2.09849 & 5.39320 \\ \mathrm{C} & .41056 & 2.94412 & 6.50526 \\ \mathrm{H} & .68152 & 4.96100 & 7.22211 \\ \mathrm{H} & 1.11302 & 5.88317 & 4.94907 \\ \mathrm{H} & 1.03538 & 4.41340 & 2.98316 \\ \mathrm{H} & .25861 & 1.03424 & 5.51856 \\ \mathrm{H} & .22603 & 2.52976 & 7.49314 \\ \mathrm{H} & 1.10247 & -1.55178 & 2.42002 \\ \mathrm{C} & -.52714 & -.41945 & .64512 \\ \mathrm{H} & -1.09601 & .30951 & .06299 \\ \mathrm{H} & .45364 & -.54853 & .17266 \\ \mathrm{H} & -1.05370 & -1.38114 & .58398 \\ \mathrm{C} & -.32397 & -1.76681 & 4.01638 \\ \mathrm{H} & -.90401 & -2.53848 & 3.49463 \\ \mathrm{H} & .36637 & -2.26572 & 4.70496 \\ \mathrm{H} & -1.01538 & -1.15519 & 4.60859\end{array}$

Trans OP1
31G*(THF))

$\mathrm{E}($ B3LYP $6-31 \mathrm{G} *($ THF $))=$ $-1268.74094$

$\begin{array}{cccc}\mathrm{O} & 1.24786 & .12147 & 3.69670 \\ \mathrm{C} & .48331 & -.89785 & 3.06133 \\ \mathrm{C} & -.36483 & -.01231 & 2.14259 \\ \mathrm{P} & .70251 & 1.50471 & 2.60375 \\ \mathrm{H} & -1.36055 & .16471 & 2.57399 \\ \mathrm{C} & 4.92802 & 1.93487 & .63160 \\ \mathrm{C} & 4.54792 & .73842 & 1.24352 \\ \mathrm{C} & 3.28364 & .61458 & 1.82530 \\ \mathrm{C} & 2.36208 & 1.67511 & 1.78253 \\ \mathrm{C} & 2.75796 & 2.87241 & 1.16012 \\ \mathrm{C} & 4.03031 & 3.00514 & .60003 \\ \mathrm{H} & 5.91620 & 2.03511 & .18870 \\ \mathrm{H} & 5.23949 & -.09999 & 1.28334 \\ \mathrm{H} & 3.02184 & -.29903 & 2.34335 \\ \mathrm{H} & 2.07421 & 3.71168 & 1.10234 \\ \mathrm{H} & 4.31579 & 3.94622 & .13601 \\ \mathrm{C} & -2.16389 & 4.10263 & -.17124 \\ \mathrm{C} & -1.05029 & 3.47154 & -.73061 \\ \mathrm{C} & -.15426 & 2.77152 & .08390 \\ \mathrm{C} & -.32963 & 2.69325 & 1.47758 \\ \mathrm{C} & -1.46983 & 3.31946 & 2.01364 \\ \mathrm{C} & -2.37391 & 4.01682 & 1.20729 \\ \mathrm{H} & -2.86135 & 4.64954 & -.80157 \\ \mathrm{H} & -.87488 & 3.52106 & -1.80338 \\ \mathrm{H} & .69515 & 2.28322 & -.38564 \\ \mathrm{H} & -1.66015 & 3.27326 & 3.08304\end{array}$ 


$\begin{array}{cccc}\mathrm{H} & -3.24059 & 4.49516 & 1.65910 \\ \mathrm{C} & .58679 & 4.30034 & 6.37036 \\ \mathrm{C} & .94581 & 4.80740 & 5.11798 \\ \mathrm{C} & .94399 & 3.97502 & 3.99778 \\ \mathrm{C} & .61980 & 2.61253 & 4.11179 \\ \mathrm{C} & .27051 & 2.11330 & 5.37513 \\ \mathrm{C} & .23993 & 2.95369 & 6.49256 \\ \mathrm{H} & .57978 & 4.95054 & 7.24220 \\ \mathrm{H} & 1.22211 & 5.85368 & 5.01037 \\ \mathrm{H} & 1.20000 & 4.39867 & 3.03236 \\ \mathrm{H} & .04566 & 1.05996 & 5.48756 \\ \mathrm{H} & -.04249 & 2.54745 & 7.46124 \\ \mathrm{H} & 1.13358 & -1.55406 & 2.45505 \\ \mathrm{C} & -.50348 & -.43921 & .68484 \\ \mathrm{H} & -1.08129 & .28003 & .09884 \\ \mathrm{H} & .47565 & -.56663 & .20803 \\ \mathrm{H} & -1.02453 & -1.40479 & .63919 \\ \mathrm{C} & -.28319 & -1.75897 & 4.06006 \\ \mathrm{H} & -.88059 & -2.51710 & 3.53773 \\ \mathrm{H} & .40665 & -2.27856 & 4.73479 \\ \mathrm{H} & -.95966 & -1.14287 & 4.66516\end{array}$

\section{Cis OP2 (B3LYP 6-31G*)}

$\mathrm{E}($ B3LYP 6-31G*) $=-1268.71993$

$\mathrm{E}(\mathrm{B} 3 \mathrm{LYP} 6-31 \mathrm{G} *(\mathrm{THF}))=$ $-1268.72799$

$\begin{array}{cccc}\mathrm{O} & .28243 & .22338 & -.06836 \\ \mathrm{C} & .08170 & .29816 & 1.36949 \\ \mathrm{C} & 1.54000 & .13212 & 1.80253 \\ \mathrm{P} & 1.98417 & .11422 & -.16105 \\ \mathrm{H} & -.53922 & -.55989 & 1.65814 \\ \mathrm{H} & 1.69896 & -.87389 & 2.20549 \\ \mathrm{C} & 1.64907 & -.18411 & -4.89288 \\ \mathrm{C} & .52079 & -.43151 & -4.11206 \\ \mathrm{C} & .59023 & -.34155 & -2.71765 \\ \mathrm{C} & 1.78911 & .00015 & -2.07152 \\ \mathrm{C} & 2.91627 & .23888 & -2.87699 \\ \mathrm{C} & 2.85181 & .15176 & -4.26735 \\ \mathrm{H} & 1.59365 & -.25051 & -5.97659 \\ \mathrm{H} & -. .42276 & -.69337 & -4.58533 \\ \mathrm{H} & -.29775 & -.52825 & -2.12654 \\ \mathrm{H} & 3.86467 & .50479 & -2.41672 \\ \mathrm{H} & 3.74077 & .34822 & -4.86197 \\ \mathrm{C} & 4.20748 & 4.21751 & -.27350 \\ \mathrm{C} & 4.92467 & 3.07449 & .08034 \\ \mathrm{C} & 4.29401 & 1.82793 & .11754 \\ \mathrm{C} & 2.93793 & 1.70904 & -.21139 \\ \mathrm{C} & 2.22604 & 2.86208 & -.57684 \\ \mathrm{C} & 2.85435 & 4.10724 & -.60137 \\ \mathrm{H} & 4.69962 & 5.18608 & -.29965 \\ \mathrm{H} & 5.98106 & 3.14780 & .32630 \\ \mathrm{H} & 4.87281 & .94989 & .38630 \\ \mathrm{H} & 1.17948 & 2.78190 & -.85952 \\ \mathrm{H} & 2.28802 & 4.99004 & -.88691 \\ \mathrm{C} & 4.80074 & -3.62555 & .24643 \\ \mathrm{C} & 3.87937 & -3.55546 & -.79745\end{array}$

$\begin{array}{cccc}\mathrm{C} & 3.06608 & -2.42921 & -.94687 \\ \mathrm{C} & 3.14096 & -1.36194 & -.03949 \\ \mathrm{C} & 4.04403 & -1.46787 & 1.03349 \\ \mathrm{C} & 4.88323 & -2.57362 & 1.16187 \\ \mathrm{H} & 5.44377 & -4.49516 & .35322 \\ \mathrm{H} & 3.79435 & -4.37464 & -1.50677 \\ \mathrm{H} & 2.37009 & -2.38633 & -1.77698 \\ \mathrm{H} & 4.09474 & -.68287 & 1.78106 \\ \mathrm{H} & 5.59113 & -2.61967 & 1.98548 \\ \mathrm{C} & -.64516 & 1.58623 & 1.73309 \\ \mathrm{H} & -.80598 & 1.64655 & 2.81607 \\ \mathrm{H} & -.07807 & 2.46845 & 1.42042 \\ \mathrm{H} & -1.62246 & 1.61711 & 1.23982 \\ \mathrm{C} & 2.12361 & 1.15600 & 2.77223 \\ \mathrm{H} & 3.18078 & .95336 & 2.97958 \\ \mathrm{H} & 2.06364 & 2.17960 & 2.39071 \\ \mathrm{H} & 1.59685 & 1.12796 & 3.73675\end{array}$

Cis OP2 (B3LYP 6-31G*(THF))

$\mathrm{E}(\mathrm{B} 3 \mathrm{LYP} 6-31 \mathrm{G} *(\mathrm{THF}))=$ $-1268.73151$

$\begin{array}{cccc}\mathrm{O} & .27966 & .227668 & -.07405 \\ \mathrm{C} & .07901 & .299817 & 1.36799 \\ \mathrm{C} & 1.53797 & .134899 & 1.79612 \\ \mathrm{P} & 1.98656 & .119986 & -.16089 \\ \mathrm{H} & -.53784 & -.560321 & 1.65663 \\ \mathrm{H} & 1.69742 & -.871477 & 2.19708 \\ \mathrm{C} & 1.66326 & -.193104 & -4.90215 \\ \mathrm{C} & .54014 & -.469348 & -4.12119 \\ \mathrm{C} & .60713 & -.375080 & -2.72590 \\ \mathrm{C} & 1.79473 & .000773 & -2.07672 \\ \mathrm{C} & 2.91575 & .266919 & -2.88280 \\ \mathrm{C} & 2.85656 & .175356 & -4.27435 \\ \mathrm{H} & 1.61115 & -.262319 & -5.98647 \\ \mathrm{H} & -.39644 & -.757157 & -4.59464 \\ \mathrm{H} & -.27748 & -.585990 & -2.13753 \\ \mathrm{H} & 3.85597 & .559132 & -2.42116 \\ \mathrm{H} & 3.74149 & .394174 & -4.86834 \\ \mathrm{C} & 4.20982 & 4.228033 & -.26205 \\ \mathrm{C} & 4.93208 & 3.079520 & .06773 \\ \mathrm{C} & 4.30069 & 1.832145 & .09791 \\ \mathrm{C} & 2.93933 & 1.716992 & -.21306 \\ \mathrm{C} & 2.22264 & 2.875555 & -.55288 \\ \mathrm{C} & 2.85097 & 4.121923 & -.57188 \\ \mathrm{H} & 4.70183 & 5.197485 & -.28277 \\ \mathrm{H} & 5.99232 & 3.148885 & .30059 \\ \mathrm{H} & 4.88387 & .951682 & .34891 \\ \mathrm{H} & 1.17058 & 2.803469 & -.81755 \\ \mathrm{H} & 2.27947 & 5.008277 & -.83777 \\ \mathrm{C} & 4.77953 & -3.643280 & .25506 \\ \mathrm{C} & 3.84642 & -3.574924 & -.78009 \\ \mathrm{C} & 3.04432 & -2.439708 & -.93312 \\ \mathrm{C} & 3.14045 & -1.361428 & -.03963 \\ \mathrm{C} & 4.05687 & -1.464025 & 1.02400 \\ \mathrm{C} & 4.88469 & -2.579409 & 1.15615 \\ \mathrm{H} & 5.41323 & -4.519763 & .36614\end{array}$

$\begin{array}{cccc}\mathrm{H} & 3.74255 & -4.402723 & -1.47765 \\ \mathrm{H} & 2.33616 & -2.403323 & -1.75364 \\ \mathrm{H} & 4.13163 & -.672638 & 1.76344 \\ \mathrm{H} & 5.60198 & -2.622881 & 1.97234 \\ \mathrm{C} & -.64841 & 1.585153 & 1.73902 \\ \mathrm{H} & -.79970 & 1.639477 & 2.82393 \\ \mathrm{H} & -.08732 & 2.471251 & 1.42528 \\ \mathrm{H} & -1.63181 & 1.615372 & 1.25699 \\ \mathrm{C} & 2.12205 & 1.157169 & 2.76719 \\ \mathrm{H} & 3.18172 & .958864 & 2.96554 \\ \mathrm{H} & 2.05217 & 2.181971 & 2.39024 \\ \mathrm{H} & 1.59996 & 1.120774 & 3.73407\end{array}$

Trans OP2 (B3LYP 6-31G*)

$\mathrm{E}\left(\mathrm{B} 3 \mathrm{LYP} 6-31 \mathrm{G}^{*}\right)=-1268.72298$ $\mathrm{E}(\mathrm{B} 3 \mathrm{LYP} 6-31 \mathrm{G} *(\mathrm{THF}))=$ $-1268.73146$

$\begin{array}{cccc}\mathrm{O} & .30477 & .35650 & -.10991 \\ \mathrm{C} & .10810 & .41713 & 1.32274 \\ \mathrm{C} & 1.51234 & .01879 & 1.75862 \\ \mathrm{P} & 2.00454 & .08945 & -.18038 \\ \mathrm{H} & -.11930 & 1.45753 & 1.59720 \\ \mathrm{H} & 1.53456 & -1.02820 & 2.08713 \\ \mathrm{C} & 1.63339 & -.29416 & -4.89246 \\ \mathrm{C} & .52485 & -.57275 & -4.09353 \\ \mathrm{C} & .60782 & -.45642 & -2.70230 \\ \mathrm{C} & 1.80241 & -.06246 & -2.08021 \\ \mathrm{C} & 2.91116 & .20407 & -2.90041 \\ \mathrm{C} & 2.83038 & .09717 & -4.28877 \\ \mathrm{H} & 1.56652 & -.37854 & -5.97423 \\ \mathrm{H} & -. .11357 & -.87768 & -4.55075 \\ \mathrm{H} & -.26616 & -.66023 & -2.09561 \\ \mathrm{H} & 3.85468 & .50851 & -2.45485 \\ \mathrm{H} & 3.70265 & .32001 & -4.89855 \\ \mathrm{C} & 4.12472 & 4.23638 & -.18913 \\ \mathrm{C} & 4.90153 & 3.08836 & -.03101 \\ \mathrm{C} & 4.30328 & 1.82571 & -.02769 \\ \mathrm{C} & 2.91730 & 1.69838 & -.18778 \\ \mathrm{C} & 2.14312 & 2.85735 & -.35414 \\ \mathrm{C} & 2.74299 & 4.11662 & -.35188 \\ \mathrm{H} & 4.59245 & 5.21733 & -.18990 \\ \mathrm{H} & 5.97890 & 3.17082 & .08800 \\ \mathrm{H} & 4.92531 & .94387 & .08848 \\ \mathrm{H} & 1.06985 & 2.76745 & -.49914 \\ \mathrm{H} & 2.12984 & 5.00452 & -.48295 \\ \mathrm{C} & 4.94214 & -3.57096 & .23638 \\ \mathrm{C} & 3.99407 & -3.54675 & -.78515 \\ \mathrm{C} & 3.15385 & -2.44117 & -.94249 \\ \mathrm{C} & 3.22889 & -1.34645 & -.06826 \\ \mathrm{C} & 4.16363 & -1.40517 & .98132 \\ \mathrm{C} & 5.02637 & -2.49174 & 1.11941 \\ \mathrm{H} & 5.60516 & -4.42451 & .35020 \\ \mathrm{H} & 3.90835 & -4.38591 & -1.47076 \\ \mathrm{H} & 2.43570 & -2.43737 & -1.75503 \\ \mathrm{H} & 4.22689 & -.59484 & 1.69965 \\ \mathrm{H} & 5.75738 & -2.49963 & 1.92400\end{array}$




\begin{abstract}
$\begin{array}{llll}\text { C } & 2.15872 & .90753 & 2.81825\end{array}$
$\begin{array}{llll}\text { H } & 3.17994 & .59459 & 3.06056\end{array}$

$\begin{array}{llll}\mathrm{H} & 2.19903 & 1.95465 & 2.49955\end{array}$

$\begin{array}{llll}\mathrm{H} & 1.58366 & .86742 & 3.75459\end{array}$

$\begin{array}{llll}\text { C }-1.03933 & -.48954 & 1.74277\end{array}$

$\begin{array}{llll}\mathrm{H} & -1.18507 & -.44346 & 2.82883\end{array}$

$\begin{array}{llll}\mathrm{H} & -1.97494 & -.18797 & 1.25914\end{array}$

$\begin{array}{llll}\mathrm{H} & -.82760 & -1.52875 & 1.46569\end{array}$
\end{abstract}

\section{Cis TS2 (B3LYP 6-31G*)}

$\mathrm{E}(\mathrm{B} 3 \mathrm{LYP}$ 6-31G*) $=-1268.69191$

$\mathrm{E}(\mathrm{B} 3 \mathrm{LYP} 6-31 \mathrm{G} *(\mathrm{THF}))=$ $-1268.70112$

$\begin{array}{cccc}\mathrm{O} & .55881 & .31267 & -.36366 \\ \mathrm{C} & .42819 & .19514 & 1.40143 \\ \mathrm{C} & 1.77213 & .26540 & 1.88260 \\ \mathrm{P} & 2.11082 & .18400 & -.64309 \\ \mathrm{H} & -.02016 & -.79823 & 1.39238 \\ \mathrm{H} & 2.16679 & -.68417 & 2.23933 \\ \mathrm{C} & 1.89666 & .16420 & -5.32213 \\ \mathrm{C} & .71874 & .13688 & -4.57575 \\ \mathrm{C} & .76815 & .14153 & -3.17848 \\ \mathrm{C} & 1.99943 & .17507 & -2.50839 \\ \mathrm{C} & 3.17797 & .20409 & -3.27216 \\ \mathrm{C} & 3.12938 & .19780 & -4.66512 \\ \mathrm{H} & 1.85713 & .16258 & -6.40846 \\ \mathrm{H} & -.24470 & .11423 & -5.07896 \\ \mathrm{H} & -.14533 & .12443 & -2.59551 \\ \mathrm{H} & 4.14651 & .23944 & -2.77960 \\ \mathrm{H} & 4.05311 & .22254 & -5.23758 \\ \mathrm{C} & 4.66806 & 4.02827 & -.27286 \\ \mathrm{C} & 5.30679 & 2.78914 & -.19930 \\ \mathrm{C} & 4.57078 & 1.60611 & -.28124 \\ \mathrm{C} & 3.17589 & 1.65036 & -.43114 \\ \mathrm{C} & 2.53954 & 2.90083 & -.51244 \\ \mathrm{C} & 3.28182 & 4.07835 & -.43197 \\ \mathrm{H} & 5.24572 & 4.94631 & -.20975 \\ \mathrm{H} & 6.38654 & 2.73857 & -.08458 \\ \mathrm{H} & 5.09029 & .65451 & -.23697 \\ \mathrm{H} & 1.46242 & 2.94801 & -.64772 \\ \mathrm{H} & 2.77444 & 5.03730 & -.49821 \\ \mathrm{C} & 4.23828 & -3.90823 & -.06225 \\ \mathrm{C} & 3.23705 & -3.74300 & -1.01887 \\ \mathrm{C} & 2.62736 & -2.49886 & -1.19044 \\ \mathrm{C} & 2.98816 & -1.40954 & -.38054 \\ \mathrm{C} & 3.97504 & -1.59519 & .60133 \\ \mathrm{C} & 4.61024 & -2.82713 & . .74217 \\ \mathrm{H} & 4.72251 & -4.87306 & .06185 \\ \mathrm{H} & 2.93457 & -4.57840 & -1.64458 \\ \mathrm{H} & 1.86967 & -2.37821 & -1.95852 \\ \mathrm{H} & 4.23260 & -.77900 & 1.26649 \\ \mathrm{H} & 5.38380 & -2.94819 & 1.49574 \\ \mathrm{C} & -.57904 & 1.29079 & 1.66439 \\ \mathrm{H} & -.87560 & 1.25618 & 2.72151 \\ \mathrm{H} & -.15498 & 2.27983 & 1.47094 \\ \mathrm{H} & -1.47578 & 1.17062 & 1.04950\end{array}$

$\begin{array}{llll}\mathrm{C} & 2.26875 & 1.47442 & 2.64745 \\ \mathrm{H} & 3.35583 & 1.42421 & 2.79222 \\ \mathrm{H} & 2.07360 & 2.41855 & 2.12416 \\ \mathrm{H} & 1.81900 & 1.56928 & 3.65122\end{array}$

Cis TS2 (B3LYP 6-31G*(THF))

$\mathrm{E}(\mathrm{B} 3 \mathrm{LYP}$ 6-31G*(THF) $)=$ $-1268.70399$

$\begin{array}{cccc}\mathrm{O} & .56963 & .30730 & -.36930 \\ \mathrm{C} & .45249 & .16567 & 1.40113 \\ \mathrm{C} & 1.80039 & .27459 & 1.86646 \\ \mathrm{P} & 2.12754 & .18973 & -.64569 \\ \mathrm{H} & .03683 & -.84189 & 1.39158 \\ \mathrm{H} & 2.23336 & -.66158 & 2.21276 \\ \mathrm{C} & 1.94116 & .17072 & -5.33377 \\ \mathrm{C} & .75564 & .15991 & -4.59706 \\ \mathrm{C} & .79471 & .16465 & -3.19836 \\ \mathrm{C} & 2.01940 & .18304 & -2.51529 \\ \mathrm{C} & 3.20544 & .19628 & -3.27021 \\ \mathrm{C} & 3.16967 & .18836 & -4.66465 \\ \mathrm{H} & 1.91144 & .16770 & -6.42097 \\ \mathrm{H} & -.20481 & .14860 & -5.10771 \\ \mathrm{H} & -.12686 & .15791 & -2.62748 \\ \mathrm{H} & 4.17078 & .21597 & -2.76992 \\ \mathrm{H} & 4.09913 & .19882 & -5.22932 \\ \mathrm{C} & 4.63928 & 4.06573 & -.23838 \\ \mathrm{C} & 5.29316 & 2.83265 & -.16889 \\ \mathrm{C} & 4.57184 & 1.64063 & -.26931 \\ \mathrm{C} & 3.17734 & 1.66987 & -.43206 \\ \mathrm{C} & 2.52647 & 2.91340 & -.51304 \\ \mathrm{C} & 3.25338 & 4.10035 & -.41499 \\ \mathrm{H} & 5.20483 & 4.99101 & -.16059 \\ \mathrm{H} & 6.37279 & 2.79295 & -.04310 \\ \mathrm{H} & 5.10602 & .69715 & -.23049 \\ \mathrm{H} & 1.45018 & 2.95310 & -.65986 \\ \mathrm{H} & 2.73411 & 5.05368 & -.48117 \\ \mathrm{C} & 4.16955 & -3.95079 & -.06022 \\ \mathrm{C} & 3.13875 & -3.77127 & -.98461 \\ \mathrm{C} & 2.56255 & -2.51056 & -1.15839 \\ \mathrm{C} & 2.98722 & -1.41670 & -.38415 \\ \mathrm{C} & 4.00371 & -1.61537 & .56522 \\ \mathrm{C} & 4.60340 & -2.86618 & .70972 \\ \mathrm{H} & 4.62716 & -4.92887 & .06618 \\ \mathrm{H} & 2.78667 & -4.60830 & -1.58280 \\ \mathrm{H} & 1.77899 & -2.38467 & -1.89983 \\ \mathrm{H} & 4.31574 & -.79954 & 1.20748 \\ \mathrm{H} & 5.40001 & -2.99681 & 1.43807 \\ \mathrm{C} & -.58692 & 1.22421 & 1.68961 \\ \mathrm{H} & -.86909 & 1.16687 & 2.75011 \\ \mathrm{H} & -.20199 & 2.23043 & 1.50137 \\ \mathrm{H} & -1.49030 & 1.08066 & 1.08877 \\ \mathrm{C} & 2.27081 & 1.50031 & 2.61943 \\ \mathrm{H} & 3.36267 & 1.49247 & 2.73069 \\ \mathrm{H} & 2.02215 & 2.43440 & 2.10197 \\ \mathrm{H} & 1.84704 & 1.57934 & 3.63643 \\ & & & \end{array}$

\section{Trans TS2 (B3LYP 6-31G*)}

$\mathrm{E}\left(\mathrm{B} 3 \mathrm{LYP} 6-31 \mathrm{G}^{*}\right)=-1268.69356$ $\mathrm{E}\left(\right.$ B3LYP $\left.6-31 \mathrm{G}^{*}(\mathrm{THF})\right)=$ $-1268.70256$

$\begin{array}{cccc}\mathrm{O} & .56382 & .30752 & -.31624 \\ \mathrm{C} & .60300 & .15590 & 1.46158 \\ \mathrm{C} & 1.98487 & .12562 & 1.80610 \\ \mathrm{P} & 2.08713 & .19453 & -.72627 \\ \mathrm{H} & .07445 & 1.09211 & 1.64771 \\ \mathrm{H} & 2.35793 & -.85784 & 2.09350 \\ \mathrm{C} & 1.51962 & .44955 & -5.37104 \\ \mathrm{C} & .40292 & .47475 & -4.53589 \\ \mathrm{C} & .55618 & .38619 & -3.14887 \\ \mathrm{C} & 1.83142 & .27071 & -2.57817 \\ \mathrm{C} & 2.94779 & .24790 & -3.43098 \\ \mathrm{C} & 2.79593 & .33588 & -4.81360 \\ \mathrm{H} & 1.39942 & .51992 & -6.44913 \\ \mathrm{H} & -.59344 & .56445 & -4.96151 \\ \mathrm{H} & -.30922 & .40829 & -2.49690 \\ \mathrm{H} & 3.94939 & .16385 & -3.01630 \\ \mathrm{H} & 3.67302 & .31733 & -5.45549 \\ \mathrm{C} & 4.54960 & 4.09846 & -.39516 \\ \mathrm{C} & 5.23258 & 2.88382 & -.47258 \\ \mathrm{C} & 4.53061 & 1.67918 & -.54631 \\ \mathrm{C} & 3.12809 & 1.67896 & -.53181 \\ \mathrm{C} & 2.44557 & 2.90481 & -.46079 \\ \mathrm{C} & 3.15298 & 4.10355 & -.39254 \\ \mathrm{H} & 5.10042 & 5.03337 & -.34029 \\ \mathrm{H} & 6.31937 & 2.86937 & -.48350 \\ \mathrm{H} & 5.08233 & .74800 & -.62348 \\ \mathrm{H} & 1.35892 & 2.91502 & -.46441 \\ \mathrm{H} & 2.61031 & 5.04379 & -.33901 \\ \mathrm{C} & 4.29601 & -3.89046 & -.51668 \\ \mathrm{C} & 3.18960 & -3.70758 & -1.34575 \\ \mathrm{C} & 2.55877 & -2.46368 & -1.41171 \\ \mathrm{C} & 3.00721 & -1.39397 & -.61953 \\ \mathrm{C} & 4.10183 & -1.59798 & .23584 \\ \mathrm{C} & 4.75360 & -2.82857 & .26917 \\ \mathrm{H} & 4.79553 & -4.85454 & -.47550 \\ \mathrm{H} & 2.82063 & -4.52839 & -1.95489 \\ \mathrm{H} & 1.71721 & -2.32653 & -2.08372 \\ \mathrm{H} & 4.42727 & -.80025 & .89404 \\ \mathrm{H} & 5.60934 & -2.96429 & .92512 \\ \mathrm{C} & -.26210 & -1.07009 & 1.63152 \\ \mathrm{H} & -.51901 & -1.17791 & 2.69393 \\ \mathrm{H} & -1.19062 & -1.00368 & 1.05698 \\ \mathrm{H} & .27798 & -1.97123 & 1.32246 \\ \mathrm{C} & 2.60227 & 1.28137 & 2.56350 \\ \mathrm{H} & 3.68934 & 1.34437 & 2.41330 \\ \mathrm{H} & 2.18938 & 2.24422 & 2.23818 \\ \mathrm{H} & 2.43819 & 1.21379 & 3.65245 \\ & & & \end{array}$

Trans TS2
31G*(THF)) 


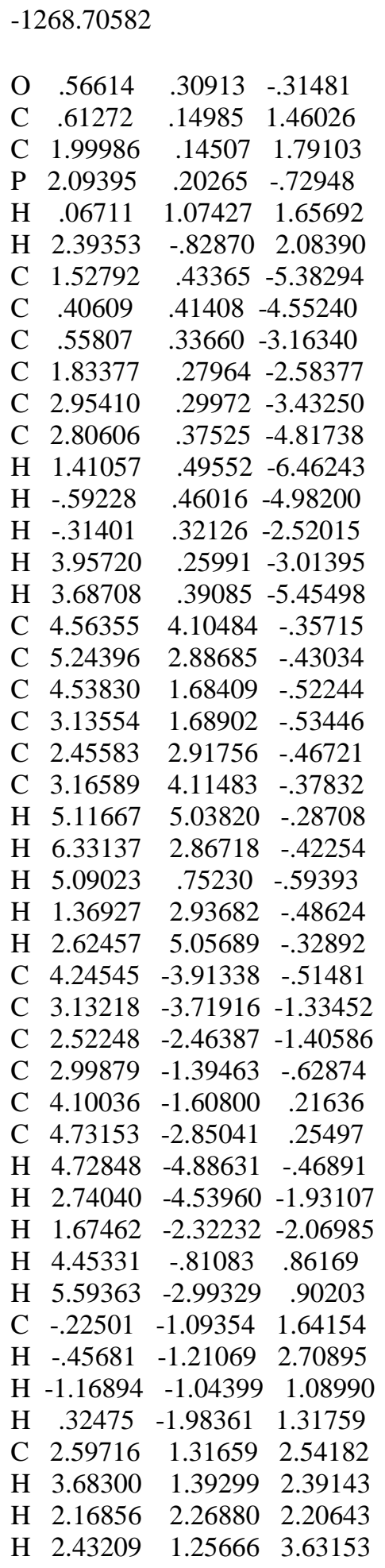

\section{POPh $_{3}$ (B3LYP 6-31G*)}

$\mathrm{E}(\mathrm{B} 3 \mathrm{LYP}$ 6-31G*) $=-1111.55062$

$\mathrm{E}\left(\mathrm{B} 3 \mathrm{LYP} 6-31 \mathrm{G}^{*}(\mathrm{THF})\right)=$ $-1111.56680$

$\begin{array}{llll}\text { O } & .51307 & -1.53831 & .01969\end{array}$ $\begin{array}{llll}\text { P } & -.03654 & -.12951 & .01838\end{array}$

$\begin{array}{lccc}\text { C } & -2.51142 & .50954 & -3.83704 \\ \text { C } & -1.95243 & 1.62454 & -3.20471 \\ \text { C } & -1.20646 & 1.46709 & -2.03361 \\ \text { C } & -1.02253 & .18900 & -1.48500 \\ \text { C } & -1.58331 & -.92762 & -2.12550 \\ \text { C } & -2.32581 & -.76654 & -3.29686 \\ \text { H } & -3.08346 & .63386 & -4.75332 \\ \text { H } & -2.08675 & 2.61622 & -3.62877 \\ \text { H } & -.75629 & 2.33842 & -1.56509 \\ \text { H } & -1.41853 & -1.92084 & -1.71820 \\ \text { H } & -2.75057 & -1.63736 & -3.79018 \\ \text { C } & 3.48684 & 2.86463 & .15048 \\ \text { C } & 2.24886 & 3.25982 & .66769 \\ \text { C } & 1.16190 & 2.38234 & .63387 \\ \text { C } & 1.30837 & 1.10452 & .07337 \\ \text { C } & 2.55416 & .71163 & -.44351 \\ \text { C } & 3.63873 & 1.58993 & -.40490 \\ \text { H } & 4.33374 & 3.54544 & .18733 \\ \text { H } & 2.13154 & 4.24682 & 1.10836 \\ \text { H } & .21110 & 2.69069 & 1.06139 \\ \text { H } & 2.67775 & -.28719 & -.85356 \\ \text { H } & 4.60196 & 1.27534 & -.79980 \\ \text { C } & -2.71457 & .44765 & 3.74698 \\ \text { C } & -2.97455 & 1.21567 & 2.60717 \\ \text { C } & -2.17885 & 1.07281 & 1.46747 \\ \text { C } & -1.11185 & .16220 & 1.46631 \\ \text { C } & -.85554 & -.60722 & 2.61354 \\ \text { C } & -1.65450 & -.46360 & 3.74931 \\ \text { H } & -3.34143 & .55335 & 4.62899 \\ \text { H } & -3.80419 & 1.91793 & 2.60032 \\ \text { H } & -2.40575 & 1.65790 & .57966 \\ \text { H } & -.04549 & -1.33095 & 2.60741 \\ \text { H } & -1.45389 & -1.06862 & 4.63056\end{array}$

\section{POPh $_{3}($ B3LYP 6-31G*(THF))}

\section{$\mathrm{E}(\mathrm{B} 3 \mathrm{LYP} 6-31 \mathrm{G} *(\mathrm{THF}))=$ $-1111.56901$}

$\begin{array}{lccc}\mathrm{O} & .51728 & -1.54430 & .02188 \\ \mathrm{P} & -.03196 & -.13795 & .01903 \\ \mathrm{C} & -2.51580 & .51322 & -3.82920 \\ \mathrm{C} & -1.95736 & 1.62678 & -3.19462 \\ \mathrm{C} & -1.20753 & 1.46583 & -2.02693 \\ \mathrm{C} & -1.01998 & .18618 & -1.48337 \\ \mathrm{C} & -1.57891 & -.92911 & -2.12754 \\ \mathrm{C} & -2.32470 & -.76471 & -3.29565 \\ \mathrm{H} & -3.09150 & .64026 & -4.74266 \\ \mathrm{H} & -2.09511 & 2.61981 & -3.61472 \\ \mathrm{H} & -.75665 & 2.33559 & -1.55594 \\ \mathrm{H} & -1.40936 & -1.92297 & -1.72371 \\ \mathrm{H} & -2.74813 & -1.63449 & -3.79211 \\ \mathrm{C} & 3.47804 & 2.87345 & .14410 \\ \mathrm{C} & 2.23745 & 3.26664 & .65409 \\ \mathrm{C} & 1.15520 & 2.38357 & .62418 \\ \mathrm{C} & 1.30870 & 1.10237 & .07438 \\ \mathrm{C} & 2.55829 & .71096 & -.43227 \\ \mathrm{C} & 3.63784 & 1.59476 & -.39783\end{array}$

H $4.32116 \quad 3.55911 \quad .17674$

$\begin{array}{llll}\mathrm{H} & 2.11450 & 4.25641 & 1.08630\end{array}$

$\mathrm{H} \quad .20152 \quad 2.68986 \quad 1.04633$

$\begin{array}{llll}\text { H } & 2.68639 & -.29108 & -.83136\end{array}$

$\begin{array}{lllll}\mathrm{H} & 4.60415 & 1.28167 & -.78568\end{array}$

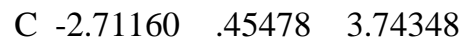

$\begin{array}{llll}\text { C } & -2.96008 & 1.23143 & 2.60823\end{array}$

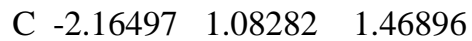

$\begin{array}{llll}\text { C } & -1.10980 & .15856 & 1.46426\end{array}$

$\begin{array}{llll}\text { C } & -.86756 & -.62225 & 2.60580\end{array}$

$\begin{array}{llll}\text { C } & -1.66539 & -.47275 & 3.74094\end{array}$

H $-3.33720 \quad .56597 \quad 4.62570$

$\begin{array}{llll}\mathrm{H} & -3.78023 & 1.94484 & 2.60448\end{array}$

$\begin{array}{llll}\mathrm{H} & -2.38205 & 1.67428 & .58334\end{array}$

$\begin{array}{llll}\mathrm{H} & -.06922 & -1.35876 & 2.59398\end{array}$

H $-1.47511-1.08583 \quad 4.61846$

\section{D. $\mathrm{Me}_{3} \mathrm{PCHPh}+$ PhCHO (reaction 3)}

PhCHO (B3LYP 6-31G*)

E(B3LYP 6-31G*)=-345.57323
$\begin{array}{ll}\text { (B3LYP } & \left.6-31 \mathrm{G}^{*}(\mathrm{THF})\right) \\ 345.67886 & \end{array}$

$\begin{array}{llll}\text { C } & 0.15654 & 2.26713 & 0.00000\end{array}$

$\begin{array}{llll}\text { C } & 1.36399 & 1.56310 & 0.00000\end{array}$

$\begin{array}{llll}\text { C } & 1.34987 & 0.16888 & 0.00000\end{array}$

$\begin{array}{llll}\text { C } & 0.13240 & -0.52397 & 0.00000\end{array}$

$\begin{array}{llll}\text { C } & -1.07784 & 0.18790 & 0.00000\end{array}$

$\begin{array}{llll}\text { C } & -1.06348 & 1.57919 & 0.00000\end{array}$

$\begin{array}{llll}\mathrm{H} & 0.16469 & 3.35416 & 0.00000\end{array}$

$\begin{array}{lllll}\mathrm{H} & 2.30904 & 2.09932 & 0.00000\end{array}$

$\begin{array}{lrrr}\mathrm{H} & 2.28350 & -0.39088 & 0.00000\end{array}$

$\begin{array}{llll}\mathrm{H} & -2.00912 & -0.37103 & 0.00000\end{array}$

$\begin{array}{llll}\mathrm{H} & -1.99826 & 2.13367 & 0.00000\end{array}$

C $0.13163 \quad-2.00603 \quad 0.00000$

$\begin{array}{llll}\text { O } & -0.86452 & -2.70263 & 0.00000\end{array}$

$\begin{array}{llll}\mathrm{H} & 1.14595 & -2.46554 & 0.00000\end{array}$

\section{PhCHO (B3LYP 6-31G*(THF))}

$\mathrm{E}(\mathrm{B} 3 \mathrm{LYP} 6-31 \mathrm{G} *(\mathrm{THF}))=$
-345.58250

$\begin{array}{llll}\text { C } & .15584 & 2.26490 & .00000\end{array}$

$\begin{array}{llll}\text { C } & 1.36351 & 1.55998 & .00000\end{array}$

$\begin{array}{llll}\text { C } & 1.34913 & .16546 & .00000\end{array}$

$\begin{array}{llll}\text { C } & .12793 & -.52392 & .00000\end{array}$

$\begin{array}{llll}\text { C }-1.08398 & .18845 & .00000\end{array}$

$\begin{array}{llll}\text { C } & -1.06742 & 1.57982 & .00000\end{array}$

$\begin{array}{llll}\mathrm{H} & .16563 & 3.35204 & .00000\end{array}$

$\begin{array}{llll}\mathrm{H} & 2.30887 & 2.09563 & .00000\end{array}$

$\begin{array}{llll}\mathrm{H} & 2.28096 & -.39710 & .00000\end{array}$

$\begin{array}{llll}\mathrm{H} & -2.02094 & -.36170 & .00000\end{array}$ 
H $-2.00118 \quad 2.13637 \quad 00000$

C $\quad .14027-1.99750 \quad .00000$

$\begin{array}{lllll}\text { O } & -.85789 & -2.70396 & .00000\end{array}$

H $1.15023 \quad-2.45625 \quad .00000$

\section{Me 3 PCHPh (B3L YP 6-31G*)}

$\mathrm{E}\left(\mathrm{B} 3 \mathrm{LYP} 6-31 \mathrm{G}^{*}\right)=-731.41515$

$\mathrm{E}(\mathrm{B} 3 \mathrm{LYP} 6-31 \mathrm{G} *(\mathrm{THF}))=$ $-731.42882$

$\begin{array}{lccl}\mathrm{C} & 1.65986 & -.24627 & .38421 \\ \mathrm{P} & 1.92446 & .48160 & -1.12161 \\ \mathrm{C} & 2.68228 & -.54898 & -2.46291 \\ \mathrm{C} & .31966 & 1.03013 & -1.80803 \\ \mathrm{C} & 2.99733 & 1.98864 & -1.13663 \\ \mathrm{H} & .61437 & -.32298 & .66784 \\ \mathrm{H} & 2.00064 & -1.37072 & -2.70222 \\ \mathrm{H} & 3.61755 & -.98339 & -2.09762 \\ \mathrm{H} & 2.88822 & .03429 & -3.36751 \\ \mathrm{H} & .46294 & 1.49789 & -2.78642 \\ \mathrm{H} & -.14326 & 1.75106 & -1.12897 \\ \mathrm{H} & -.34665 & .17006 & -1.91806 \\ \mathrm{H} & 3.18795 & 2.34691 & -2.15446 \\ \mathrm{H} & 3.95155 & 1.75851 & -.65428 \\ \mathrm{H} & 2.50637 & 2.77479 & -.55572 \\ \mathrm{C} & 4.52867 & -1.89902 & 3.13712 \\ \mathrm{C} & 4.95825 & -1.35306 & 1.92615 \\ \mathrm{C} & 4.04802 & -.81784 & 1.01769 \\ \mathrm{C} & 2.65477 & -.79741 & 1.27926 \\ \mathrm{C} & 2.24592 & -1.36026 & 2.51489 \\ \mathrm{C} & 3.15804 & -1.89592 & 3.41658 \\ \mathrm{H} & 5.24051 & -2.31850 & 3.84235 \\ \mathrm{H} & 6.01782 & -1.34893 & 1.67775 \\ \mathrm{H} & 4.43390 & -.41589 & .08192 \\ \mathrm{H} & 1.18507 & -1.36532 & 2.75830 \\ \mathrm{H} & 2.79412 & -2.31579 & 4.35227\end{array}$

\section{$\mathrm{Me}_{3} \mathrm{PCHPh}$ 31G*(THF)) \\ (B3LYP}

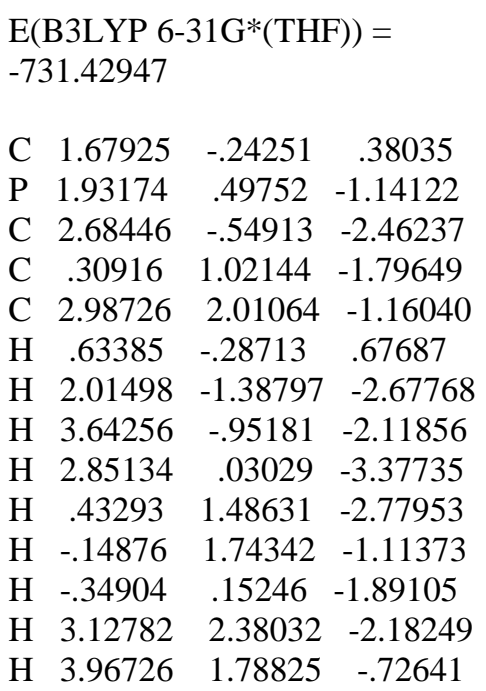

$\begin{array}{llll}\mathrm{H} & 2.50929 & 2.78574 & -.55324 \\ \mathrm{C} & 4.51759 & -1.92203 & 3.15597 \\ \mathrm{C} & 4.95866 & -1.37254 & 1.94716 \\ \mathrm{C} & 4.06141 & -.82440 & 1.03253 \\ \mathrm{C} & 2.66021 & -.79443 & 1.27912 \\ \mathrm{C} & 2.24064 & -1.36202 & 2.51628 \\ \mathrm{C} & 3.14226 & -1.90791 & 3.42406 \\ \mathrm{H} & 5.22180 & -2.34823 & 3.86614 \\ \mathrm{H} & 6.02150 & -1.36999 & 1.70944 \\ \mathrm{H} & 4.45936 & -.41411 & .10629 \\ \mathrm{H} & 1.17738 & -1.36468 & 2.75319 \\ \mathrm{H} & 2.76651 & -2.32903 & 4.35568\end{array}$

\section{Cis TS1 (B3LYP 6-31G*)}

$\mathrm{E}\left(\mathrm{B} 3 \mathrm{LYP} 6-31 \mathrm{G}^{*}\right)=-1076.97840$

$\mathrm{E}\left(\mathrm{B} 3 \mathrm{LYP} 6-31 \mathrm{G}^{*}(\mathrm{THF})\right)=$

$-1076.99793$

$\begin{array}{llll}\mathrm{O} & 1.05004 & 1.35990 & 1.68335 \\ \mathrm{C} & 1.55994 & 1.36254 & .50743 \\ \mathrm{P} & -.99388 & .13273 & .01982 \\ \mathrm{C} & .67953 & .00626 & -.61082 \\ \mathrm{C} & 3.03961 & 1.07049 & .35363 \\ \mathrm{C} & 1.25439 & -1.36989 & -.56230 \\ \mathrm{C} & 3.76496 & .63768 & 1.46775 \\ \mathrm{C} & 5.13855 & .41117 & 1.37459 \\ \mathrm{C} & 5.80346 & .62281 & .16497 \\ \mathrm{C} & 5.08704 & 1.06849 & -.95000 \\ \mathrm{C} & 3.71439 & 1.29268 & -.85191 \\ \mathrm{C} & 1.60627 & -1.97519 & .66147 \\ \mathrm{C} & 2.17024 & -3.24946 & .69231 \\ \mathrm{C} & 2.41115 & -3.94878 & -.49268 \\ \mathrm{C} & 2.09059 & -3.35274 & -1.71391 \\ \mathrm{C} & 1.51698 & -2.08238 & -1.74657 \\ \mathrm{H} & 1.27684 & 2.18946 & -.18756 \\ \mathrm{H} & .67034 & .40186 & -1.63364 \\ \mathrm{H} & 3.22778 & .51312 & 2.40309 \\ \mathrm{H} & 5.69365 & .07769 & 2.24863 \\ \mathrm{H} & 6.87472 & .45173 & .09196 \\ \mathrm{H} & 5.60131 & 1.24744 & -1.89131 \\ \mathrm{H} & 3.15915 & 1.65238 & -1.71750 \\ \mathrm{H} & 1.48006 & -1.41500 & 1.58235 \\ \mathrm{H} & 2.44090 & -3.69100 & 1.64836 \\ \mathrm{H} & 2.85383 & -4.94082 & -.46527 \\ \mathrm{H} & 2.28522 & -3.87811 & -2.64576 \\ \mathrm{H} & 1.27289 & -1.62611 & -2.70396 \\ \mathrm{C} & -1.52847 & 1.87321 & .14192 \\ \mathrm{H} & -2.57528 & 1.92214 & .45711 \\ \mathrm{H} & -.87657 & 2.33190 & .89213 \\ \mathrm{H} & -1.41733 & 2.37963 & -.82103 \\ \mathrm{C} & -2.22146 & -.70983 & -1.05637 \\ \mathrm{H} & -3.22086 & -.67728 & -.60836 \\ \mathrm{H} & -2.25374 & -.22303 & -2.03590 \\ \mathrm{H} & -1.92303 & -1.75316 & -1.19481 \\ \mathrm{H} & -1.18674 & -.59541 & 1.67658 \\ & -.98196 & -1.66780 & 1.64680\end{array}$

$\begin{array}{llll}\text { H } & -.45888 & -.08242 & 2.31238\end{array}$

\section{Cis TS1 (B3LYP 6-31G*(THF))}

\author{
$\mathrm{E}(\mathrm{B} 3 \mathrm{LYP} 6-31 \mathrm{G} *(\mathrm{THF}))=$ \\ $-1077.00030$
}

$\begin{array}{cccc}\mathrm{O} & 1.16962 & 1.49921 & 1.82637 \\ \mathrm{C} & 1.62308 & 1.48387 & .65372 \\ \mathrm{P} & -1.10796 & .10252 & .01318 \\ \mathrm{C} & .54248 & -.10310 & -.57494 \\ \mathrm{C} & 3.03592 & 1.07758 & .37145 \\ \mathrm{C} & 1.22428 & -1.40668 & -.50634 \\ \mathrm{C} & 3.79316 & .43627 & 1.36046 \\ \mathrm{C} & 5.13392 & .12539 & 1.13472 \\ \mathrm{C} & 5.73561 & .45816 & -.08289 \\ \mathrm{C} & 4.98727 & 1.10175 & -1.07482 \\ \mathrm{C} & 3.64496 & 1.40713 & -.84672 \\ \mathrm{C} & 1.38608 & -2.13603 & .69426 \\ \mathrm{C} & 2.11032 & -3.32775 & .72721 \\ \mathrm{C} & 2.71775 & -3.82861 & -.42769 \\ \mathrm{C} & 2.59275 & -3.10833 & -1.62034 \\ \mathrm{C} & 1.85759 & -1.92544 & -1.65825 \\ \mathrm{H} & 1.23155 & 2.20052 & -.09360 \\ \mathrm{H} & .60544 & .35564 & -1.56636 \\ \mathrm{H} & 3.31705 & .20818 & 2.30886 \\ \mathrm{H} & 5.71499 & -.36736 & 1.91139 \\ \mathrm{H} & 6.78408 & .22528 & -.25550 \\ \mathrm{H} & 5.45402 & 1.37315 & -2.01923 \\ \mathrm{H} & 3.06338 & 1.91916 & -1.61248 \\ \mathrm{H} & .97931 & -1.74585 & 1.62127 \\ \mathrm{H} & 2.21415 & -3.85984 & 1.67123 \\ \mathrm{H} & 3.28552 & -4.75562 & -.39802 \\ \mathrm{H} & 3.06697 & -3.47288 & -2.52983 \\ \mathrm{H} & 1.76708 & -1.37954 & -2.59549 \\ \mathrm{C} & -1.53345 & 1.87801 & -.00712 \\ \mathrm{H} & -2.58021 & 2.01458 & .28314 \\ \mathrm{H} & -.88149 & 2.38258 & .71110 \\ \mathrm{H} & -1.38177 & 2.29858 & -1.00627 \\ \mathrm{C} & -2.42200 & -.73346 & -.96790 \\ \mathrm{H} & -3.41091 & -.54800 & -.53172 \\ \mathrm{H} & -2.40685 & -.35713 & -1.99628 \\ \mathrm{H} & -2.23381 & -1.81186 & -.98693 \\ \mathrm{C} & -1.34981 & -.46153 & 1.73104 \\ \mathrm{H} & -2.33780 & -.14269 & 2.08012 \\ & -.57204 & -.00082 & 2.34484\end{array}$

\section{Trans TS1 (B3LYP 6-31G*)}

$\mathrm{E}\left(\mathrm{B} 3 \mathrm{LYP} 6-31 \mathrm{G}^{*}\right)=-1076.98301$ $\mathrm{E}(\mathrm{B} 3 \mathrm{LYP} 6-31 \mathrm{G} *(\mathrm{THF}))=$ $-1077.00365$

$\begin{array}{cccc}\mathrm{C} & .018752 & -.08356 & .59650 \\ \mathrm{O} & -.100109 & .05631 & 1.87610 \\ \mathrm{P} & 2.679417 & .22813 & 1.55732 \\ \mathrm{C} & 1.791792 & -.55259 & .19492\end{array}$ 


$\begin{array}{cccc}\text { C } & -.778338 & -1.19365 & -.07706 \\ \text { C } & -1.005666 & -1.21722 & -1.45862 \\ \text { C } & -1.732881 & -2.25722 & -2.04074 \\ \text { C } & -2.245627 & -3.28544 & -1.24581 \\ \text { C } & -2.034656 & -3.26036 & .13548 \\ \text { C } & -1.309839 & -2.21874 & .7141 \\ \text { C } & 2.152235 & 1.93584 & 1.93970 \\ \text { C } & 2.466091 & -.78400 & 3.05410 \\ \text { C } & 4.479256 & .31696 & 1.21882 \\ \text { H } & -.013526 & .83753 & -.03398 \\ \text { H } & 1.813122 & -1.62521 & .40963 \\ \text { H } & -.614803 & -.41395 & -2.07960 \\ \text { H } & -1.905128 & -2.26231 & -3.11458 \\ \text { H } & -2.813493 & -4.09503 & -1.69825 \\ \text { H } & -2.446015 & -4.04937 & .76151 \\ \text { H } & -1.157593 & -2.15924 & 1.78777 \\ \text { H } & 2.682111 & 2.28899 & 2.82994 \\ \text { H } & 1.074690 & 1.86961 & 2.12164 \\ \text { H } & 2.373389 & 2.60655 & 1.10585 \\ \text { H } & 2.899889 & -.28465 & 3.92637 \\ \text { H } & 2.946317 & -1.75769 & 2.91387 \\ \text { H } & 1.382963 & -.89687 & 3.16663 \\ \text { H } & 5.003193 & .80448 & 2.04785 \\ \text { H } & 4.651007 & .88339 & .29928 \\ \text { H } & 4.880372 & -.69151 & 1.08301 \\ \text { C } & 2.965417 & .26858 & -3.87523 \\ \text { C } & 2.878583 & -1.04582 & -3.40842 \\ \text { C } & 2.530420 & -1.30340 & -2.08415 \\ \text { C } & 2.253369 & -.25575 & -1.18522 \\ \text { C } & 2.327302 & 1.06301 & -1.67930 \\ \text { C } & 2.686818 & 1.32083 & -3.00215 \\ \text { H } & 3.237730 & .46897 & -4.90769 \\ \text { H } & 3.082835 & -1.87607 & -4.07973 \\ \text { H } & 2.458729 & -2.33062 & -1.73478 \\ \text { H } & 2.071673 & 1.89701 & -1.03109 \\ \text { H } & 2.733919 & 2.34839 & -3.35422\end{array}$

Trans TS1 (B3LYP 6-31G*(THF))

$\mathrm{E}($ B3LYP 6-31G*(THF) $)=$
-1077.00384

$\begin{array}{lccc}\mathrm{C} & -.04404 & -.03362 & .53430 \\ \mathrm{O} & -.28222 & .22318 & 1.76363 \\ \mathrm{P} & 2.76641 & .21122 & 1.58735 \\ \mathrm{C} & 1.90762 & -.55793 & .22311 \\ \mathrm{C} & -.74612 & -1.21352 & -.10575 \\ \mathrm{C} & -.99050 & -1.26357 & -1.48500 \\ \mathrm{C} & -1.68905 & -2.33645 & -2.04635 \\ \mathrm{C} & -2.15597 & -3.37328 & -1.23375 \\ \mathrm{C} & -1.92299 & -3.32731 & .14597 \\ \mathrm{C} & -1.22861 & -2.25313 & .70252 \\ \mathrm{C} & 2.28137 & 1.94483 & 1.90003 \\ \mathrm{C} & 2.34200 & -.74051 & 3.07879 \\ \mathrm{C} & 4.59101 & .21962 & 1.42058 \\ \mathrm{H} & .04323 & .81466 & -.17726 \\ \mathrm{H} & 1.90444 & -1.63245 & .42073 \\ \mathrm{H} & -.64122 & -.45204 & -2.11920\end{array}$

\begin{abstract}
$\begin{array}{llll}\mathrm{H} & -1.87830 & -2.35776 & -3.11741\end{array}$
H $\quad-2.70573 \quad-4.20508 \quad-1.66900$

$\begin{array}{llll}\mathrm{H} & -2.29541 & -4.12425 & .78695\end{array}$

$\begin{array}{llll}\mathrm{H} & -1.06802 & -2.18844 & 1.77517\end{array}$

$\begin{array}{llll}\mathrm{H} & 2.73850 & 2.28066 & 2.83724\end{array}$

$\begin{array}{llll}\mathrm{H} & 1.19159 & 1.95976 & 1.98731\end{array}$

$\begin{array}{llll}\mathrm{H} & 2.61447 & 2.60159 & 1.09196\end{array}$

$\begin{array}{llll}\mathrm{H} & 2.77476 & -.27595 & 3.97048\end{array}$

$\begin{array}{llll}\mathrm{H} & 2.71264 & -1.76649 & 2.98644\end{array}$

$\begin{array}{llll}\mathrm{H} & 1.24919 & -.73981 & 3.13861\end{array}$

$\begin{array}{llll}\mathrm{H} & 5.04866 & .72530 & 2.27853\end{array}$

$\begin{array}{llll}\mathrm{H} & 4.87196 & .74479 & .50241\end{array}$

$\begin{array}{llll}\mathrm{H} & 4.96135 & -.80880 & 1.36590\end{array}$

$\begin{array}{llll}\text { C } & 2.75918 & .31139 & -3.91339\end{array}$

$\begin{array}{llll}\text { C } & 2.65745 & -1.00857 & -3.46092\end{array}$

$\begin{array}{llll}\text { C } & 2.42447 & -1.27777 & -2.11349\end{array}$

$\begin{array}{llll}\text { C } & 2.27606 & -.23854 & -1.17023\end{array}$

$\begin{array}{llll}\text { C } & 2.37076 & 1.08654 & -1.64835\end{array}$

$\begin{array}{llll}\text { C } & 2.61622 & 1.35556 & -2.99577\end{array}$

$\begin{array}{llll}\mathrm{H} & 2.94255 & .52150 & -4.96426\end{array}$

H $\quad 2.76284 \quad-1.83440 \quad-4.16149$

$\mathrm{H} \quad 2.34723 \quad-2.30876 \quad-1.77567$

$\begin{array}{llll}\mathrm{H} & 2.22991 & 1.92136 & -.96681\end{array}$

$\begin{array}{llll}\mathrm{H} & 2.68358 & 2.38901 & -3.32970\end{array}$
\end{abstract}

\section{Cis OP1 (B3LYP 6-31G*)}

E(B3LYP 6-31G*)=-1077.00616
$\begin{aligned} & \left.\text { (B3LYP } 6-31 G^{*}(\text { THF })\right)= \\ & 1077.01675\end{aligned}$

$\begin{array}{cccc}\mathrm{O} & -.01582 & -.16604 & .08099 \\ \mathrm{C} & .02938 & -.02590 & 1.48077 \\ \mathrm{C} & 1.57889 & .12809 & 1.65263 \\ \mathrm{P} & 1.80109 & .06687 & -.24159 \\ \mathrm{C} & -.86205 & 1.08147 & 2.02022 \\ \mathrm{C} & -1.01926 & 1.25104 & 3.40300 \\ \mathrm{C} & -1.84466 & 2.25353 & 3.90957 \\ \mathrm{C} & -2.53595 & 3.09911 & 3.03745 \\ \mathrm{C} & -2.40065 & 2.92442 & 1.65966 \\ \mathrm{C} & -1.57111 & 1.91946 & 1.15459 \\ \mathrm{C} & 1.43882 & 1.46758 & -1.38759 \\ \mathrm{C} & 1.82942 & -1.55519 & -1.12460 \\ \mathrm{C} & 3.68993 & .31307 & -.23098 \\ \mathrm{H} & -.28565 & -.95969 & 1.97856 \\ \mathrm{H} & 1.99325 & -.82814 & 1.99951 \\ \mathrm{H} & -.48533 & .59567 & 4.08864 \\ \mathrm{H} & -1.94986 & 2.37524 & 4.98482 \\ \mathrm{H} & -3.18195 & 3.88004 & 3.43060 \\ \mathrm{H} & -2.94725 & 3.56786 & .97413 \\ \mathrm{H} & -1.47602 & 1.75891 & .08571 \\ \mathrm{H} & 2.25552 & 1.61861 & -2.09939 \\ \mathrm{H} & 1.32091 & 2.38474 & -.80189 \\ \mathrm{H} & .49949 & 1.27255 & -1.90790 \\ \mathrm{H} & 2.68829 & -1.64081 & -1.79597 \\ \mathrm{H} & .89254 & -1.68076 & -1.66991 \\ \mathrm{H} & 1.88914 & -2.35425 & -.37741 \\ \mathrm{H} & 3.95951 & 1.28568 & .19417\end{array}$
H 4.10012
$.25506-1.24627$
$\begin{array}{llll}\text { H } & 4.17752 & -.45928 & .37728\end{array}$
C $3.57135 \quad 3.221113 .93330$
$\begin{array}{llll}\text { C } & 3.86826 & 1.87399 & 4.14343\end{array}$
$\begin{array}{llll}\text { C } & 3.20720 & .89098 & 3.40693\end{array}$
$\begin{array}{llll}\text { C } & 2.23904 & 1.22423 & 2.44655\end{array}$
$\begin{array}{llll}\text { C } & 1.94908 & 2.58597 & 2.25038\end{array}$
$\begin{array}{llll}\text { C } & 2.60789 & 3.57111 & 2.98517\end{array}$
H $4.08198 \quad 3.99129 \quad 4.50526$
H $4.61229 \quad 1.58559 \quad 4.88149$
H $3.44442 \quad-.15703 \quad 3.57865$
$\begin{array}{llll}\mathrm{H} & 1.18672 & 2.88080 & 1.53665\end{array}$
$\begin{array}{llll}\mathrm{H} & 2.36255 & 4.61683 & 2.81850\end{array}$

\section{Trans OP1 (B3LYP 6-31G*)}

$\mathrm{E}\left(\mathrm{B} 3 \mathrm{LYP} 6-31 \mathrm{G}^{*}\right)=-1077.01047$ $\mathrm{E}\left(\right.$ B3LYP $\left.6-31 \mathrm{G}^{*}(\mathrm{THF})\right)=$ $-1077.02163$

$\begin{array}{cccc}\mathrm{O} & -.19271 & -.00042 & -.32594 \\ \mathrm{C} & .28037 & .06757 & .99878 \\ \mathrm{C} & 1.73670 & .51460 & .70576 \\ \mathrm{P} & 1.47195 & .14953 & -1.14846 \\ \mathrm{C} & -.50361 & 1.01903 & 1.88585 \\ \mathrm{C} & -.27396 & 1.04224 & 3.26826 \\ \mathrm{C} & -.96348 & 1.93518 & 4.08815 \\ \mathrm{C} & -1.90072 & 2.81273 & 3.53590 \\ \mathrm{C} & -2.14189 & 2.78605 & 2.16147 \\ \mathrm{C} & -1.44694 & 1.89460 & 1.34097 \\ \mathrm{C} & 1.37839 & -1.58821 & -1.75652 \\ \mathrm{C} & .72160 & 1.35764 & -2.33015 \\ \mathrm{C} & 3.26733 & .46915 & -1.69764 \\ \mathrm{H} & .28192 & -.92394 & 1.48431 \\ \mathrm{H} & 1.79972 & 1.60928 & .76571 \\ \mathrm{H} & .44966 & .35563 & 3.70398 \\ \mathrm{H} & -.77357 & 1.94368 & 5.15868 \\ \mathrm{H} & -2.44306 & 3.50567 & 4.17428 \\ \mathrm{H} & -2.87823 & 3.45747 & 1.72600 \\ \mathrm{H} & -1.63691 & 1.85155 & .27347 \\ \mathrm{H} & .33672 & -1.84280 & -1.95724 \\ \mathrm{H} & 1.74990 & -2.25633 & -.97220 \\ \mathrm{H} & 1.99707 & -1.73378 & -2.64668 \\ \mathrm{H} & 1.29730 & 1.43071 & -3.25691 \\ \mathrm{H} & .70529 & 2.34236 & -1.85020 \\ \mathrm{H} & -.31105 & 1.06878 & -2.53305 \\ \mathrm{H} & 3.38396 & .31471 & -2.77689 \\ \mathrm{H} & 3.96393 & -.19658 & -1.17699 \\ \mathrm{H} & 3.56310 & 1.50120 & -1.46984 \\ \mathrm{C} & 5.11789 & -1.17380 & 2.80866 \\ \mathrm{C} & 5.06377 & .19360 & 2.53399 \\ \mathrm{C} & 3.96492 & .72956 & 1.86261 \\ \mathrm{C} & 2.90019 & -.08360 & 1.44490 \\ \mathrm{C} & 2.96775 & -1.45747 & 1.73328 \\ \mathrm{C} & 4.06340 & -1.99634 & 2.40711 \\ \mathrm{H} & 5.97079 & -1.59419 & 3.33459 \\ \mathrm{H} & 5.87458 & .84608 & 2.84737 \\ \mathrm{H} & 3.92688 & 1.79770 & 1.66027\end{array}$


$\begin{array}{llll}\mathrm{H} & 2.15215 & -2.11328 & 1.44033\end{array}$

H $4.09152-3.06166 \quad 2.62096$

\section{Cis OP2 (B3LYP 6-31G*)}

$\mathrm{E}\left(\mathrm{B} 3 \mathrm{LYP} 6-31 \mathrm{G}^{*}\right)=-1077.00188$

$\mathrm{E}(\mathrm{B} 3 \mathrm{LYP} 6-31 \mathrm{G} *(\mathrm{THF}))=$ $-1077.01036$

$\begin{array}{cccc}\mathrm{O} & .18159 & .00441 & .04236 \\ \mathrm{C} & .14677 & .04550 & 1.48655 \\ \mathrm{C} & 1.66103 & .17683 & 1.75351 \\ \mathrm{P} & 1.86833 & .09857 & -.27458 \\ \mathrm{C} & -.82115 & 1.08932 & 2.00392 \\ \mathrm{C} & -1.04773 & 1.19617 & 3.38339 \\ \mathrm{C} & -1.95699 & 2.12501 & 3.88531 \\ \mathrm{C} & -2.65860 & 2.96187 & 3.01332 \\ \mathrm{C} & -2.44689 & 2.85395 & 1.63870 \\ \mathrm{C} & -1.53731 & 1.91966 & 1.13564 \\ \mathrm{C} & 1.38622 & .04587 & -2.10572 \\ \mathrm{C} & 2.91394 & -1.43245 & -.27346 \\ \mathrm{C} & 3.03617 & 1.53762 & -.46254 \\ \mathrm{H} & -.20621 & -.93410 & 1.83848 \\ \mathrm{H} & 2.04237 & -.77093 & 2.14946 \\ \mathrm{H} & -.50313 & .55021 & 4.06885 \\ \mathrm{H} & -2.11714 & 2.19732 & 4.95808 \\ \mathrm{H} & -3.36801 & 3.68671 & 3.40413 \\ \mathrm{H} & -2.99505 & 3.49410 & .95178 \\ \mathrm{H} & -1.38181 & 1.82143 & .06656 \\ \mathrm{H} & 2.27642 & .00499 & -2.74283 \\ \mathrm{H} & .80927 & .93801 & -2.37249 \\ \mathrm{H} & .76123 & -.82820 & -2.31238 \\ \mathrm{H} & 3.37727 & -1.59814 & -1.25032 \\ \mathrm{H} & 2.27434 & -2.29256 & -.04506 \\ \mathrm{H} & 3.68681 & -1.36491 & .49725 \\ \mathrm{H} & 2.46998 & 2.47487 & -.43448 \\ \mathrm{H} & 3.55152 & 1.47775 & -1.42558 \\ \mathrm{H} & 3.75932 & 1.56201 & .35540 \\ \mathrm{C} & 3.52346 & 3.31265 & 4.08425 \\ \mathrm{C} & 3.90971 & 1.97979 & 4.22875 \\ \mathrm{C} & 3.27841 & .98412 & 3.48303 \\ \mathrm{C} & 2.25318 & 1.28440 & 2.56766 \\ \mathrm{C} & 1.88190 & 2.63677 & 2.43323 \\ \mathrm{C} & 2.50510 & 3.63259 & 3.18333 \\ \mathrm{H} & 4.00765 & 4.09220 & 4.66649 \\ \mathrm{H} & 4.69755 & 1.71095 & 4.92824 \\ \mathrm{H} & 3.58096 & -.05337 & 3.61340 \\ \mathrm{H} & 1.09211 & 2.91474 & 1.74296 \\ \mathrm{H} & 2.19132 & 4.66654 & 3.06295 \\ & & & \\ & & & \end{array}$

\section{Trans OP2 (B3LYP 6-31G*)}

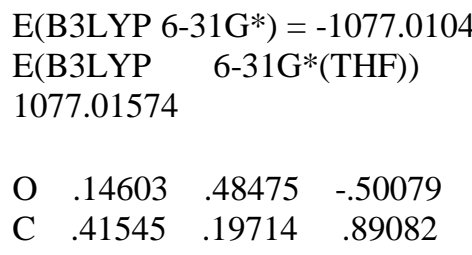

\begin{abstract}
$\begin{array}{llll}\text { C } & 1.92174 & .44993 & .89113\end{array}$
$\begin{array}{llll}\mathrm{P} & 1.74269 & .66043 & -1.12207\end{array}$

$\begin{array}{llll}\text { C } & -.40790 & 1.06567 & 1.82097\end{array}$

$\begin{array}{llll}\text { C } & -1.40358 & 1.92020 & 1.33849\end{array}$

$\begin{array}{llll}\text { C } & -2.14599 & 2.70920 & 2.22116\end{array}$

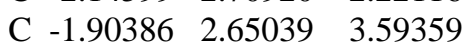

$\begin{array}{llll}\text { C } & -.91200 & 1.79536 & 4.08222\end{array}$

$\begin{array}{llll}\text { C } & -.16992 & 1.00941 & 3.20205\end{array}$

$\begin{array}{llll}\mathrm{H} & .17085 & -.85642 & 1.07898\end{array}$

$\begin{array}{llll}\mathrm{H} & 2.12546 & 1.45268 & 1.28408\end{array}$

$\begin{array}{llll}\mathrm{H} & -1.59054 & 1.95945 & .27059\end{array}$

H $-2.91644 \quad 3.37085 \quad 1.83267$

H $-2.48296 \quad 3.26428 \quad 4.27862$

$\begin{array}{llll}\mathrm{H} & -.71489 & 1.74295 & 5.15008\end{array}$

$\begin{array}{llll}\mathrm{H} & .60378 & .34856 & 3.58707\end{array}$

$\begin{array}{llll}\text { C } & 4.77411 & -2.29590 & 2.63445\end{array}$

$\begin{array}{llll}\text { C } & 5.03440 & -.92513 & 2.58228\end{array}$

$\begin{array}{llll}\text { C } & 4.09358 & -.05224 & 2.03748\end{array}$

$\begin{array}{llll}\text { C } & 2.87132 & -.51812 & 1.51729\end{array}$

$\begin{array}{llll}\text { C } & 2.62825 & -1.90334 & 1.57955\end{array}$

$\begin{array}{llll}\text { C } & 3.56326 & -2.77734 & 2.13269\end{array}$

H $\quad 5.50105 \quad-2.97856 \quad 3.06585$

$\begin{array}{llll}\mathrm{H} & 5.96781 & -.53148 & 2.97694\end{array}$

H $4.29999 \quad 1.01622 \quad 2.02116$

H $1.69590 \quad-2.30801 \quad 1.19446$

$\begin{array}{llll}\mathrm{H} & 3.34268 & -3.84123 & 2.17250\end{array}$

$\begin{array}{llll}\text { C } & 2.65647 & -.84484 & -1.68086\end{array}$

$\begin{array}{llll}\mathrm{H} & 2.95644 & -.73329 & -2.72679\end{array}$

H $3.53300-1.03013-1.05758$

H $1.99208-1.71266-1.60576$

$\begin{array}{llll}\text { C } & 2.95819 & 2.07754 & -1.18193\end{array}$

$\begin{array}{llll}\text { H } & 3.29595 & 2.28674 & -2.20173\end{array}$

$\begin{array}{llll}\mathrm{H} & 2.46613 & 2.97590 & -.79092\end{array}$

$\begin{array}{llll}\mathrm{H} & 3.81550 & 1.85331 & -.54016\end{array}$

$\begin{array}{llll}\text { C } & .92026 & 1.08151 & -2.77150\end{array}$

H $1.67085 \quad 1.19267 \quad-3.56156$

H $\quad .21258 \quad .29833 \quad-3.05938$

$\begin{array}{llll}\mathrm{H} & .36270 & 2.02050 & -2.68745\end{array}$
\end{abstract}

\section{Cis TS2 (B3LYP 6-31G*)}

$\mathrm{E}(\mathrm{B} 3 \mathrm{LYP}$ 6-31G*) $=-1076.98172$

$\mathrm{E}(\mathrm{B} 3 \mathrm{LYP} 6-31 \mathrm{G} *(\mathrm{THF}))=$

$-1076.99394$
$\begin{array}{llll}\mathrm{O} & .12178 & .14763 & .07117\end{array}$
$\begin{array}{llll}\text { C } & .06035 & .00673 & 1.76293\end{array}$
$\begin{array}{llll}\text { C } & 1.41361 & -.03731 & 2.25446\end{array}$
$\begin{array}{llll}\mathrm{P} & 1.58893 & -.25237 & -.38963\end{array}$
$\begin{array}{llll}\text { C } & -.94393 & 1.06156 & 2.17409\end{array}$
C $-1.08978 \quad 1.31290 \quad 3.54709$
C $-2.05622 \quad 2.20446 \quad 4.01003$
$\begin{array}{llll}\text { C } & -2.89065 & 2.86737 & 3.10747\end{array}$
C $-2.75180 \quad 2.62463 \quad 1.74085$
C $-1.79017 \quad 1.72411 \quad 1.27609$
$\begin{array}{llll}\text { C } & 3.00908 & .89405 & -.26059\end{array}$
$\begin{array}{llll}\text { C } & 1.26918 & -.26297 & -2.20292\end{array}$
$\begin{array}{llll}\text { C } & 2.14836 & -1.97188 & -.07619\end{array}$

\begin{abstract}
$\begin{array}{llll}\mathrm{H} & -. .40901 & -.97958 & 1.79281\end{array}$
H $1.73534 \quad-1.01560 \quad 2.60835$

$\begin{array}{lllll}\mathrm{H} & -.43072 & .81064 & 4.24928\end{array}$

$\begin{array}{llll}\mathrm{H} & -2.15042 & 2.38810 & 5.07725\end{array}$

H $-3.64027 \quad 3.567793 .46680$

H $-3.39568 \quad 3.13515 \quad 1.02876$

$\begin{array}{llll}\mathrm{H} & -1.68764 & 1.53630 & .21387\end{array}$

H $3.52426 \quad .95758$ - 1.22585

$\begin{array}{llll}\mathrm{H} & 3.69729 & .56864 & .52096\end{array}$

$\begin{array}{llll}\mathrm{H} & 2.64811 & 1.88650 & .02382\end{array}$

$\begin{array}{llll}\mathrm{H} & 2.16937 & -.54927 & -2.75838\end{array}$

H $\quad .95714 \quad .73465 \quad-2.52594$

$\mathrm{H} \quad .46454 \quad-.96507 \quad-2.43794$

$\begin{array}{llll}\mathrm{H} & 2.84978 & -2.00672 & .75735\end{array}$

$\begin{array}{llll}\text { H } & 2.61601 & -2.38339 & -.97896\end{array}$

$\begin{array}{lllll}\mathrm{H} & 1.27574 & -2.58335 & .17216\end{array}$

C $3.84483 \quad 3.172963 .79352$

$\begin{array}{llll}\text { C } & 4.14682 & 1.83309 & 4.05510\end{array}$

$\begin{array}{llll}\text { C } & 3.33489 & .81380 & 3.56643\end{array}$

$\begin{array}{llll}\text { C } & 2.18171 & 1.07903 & 2.78647\end{array}$

$\begin{array}{llll}\text { C } & 1.90216 & 2.44559 & 2.52758\end{array}$

C $2.71353 \quad 3.46255 \quad 3.02686$

H $4.47497 \quad 3.97035 \quad 4.17795$

H $5.02022 \quad 1.57917 \quad 4.65253$

$\begin{array}{llll}\mathrm{H} & 3.58302 & -.22207 & 3.79417\end{array}$

$\begin{array}{llll}\mathrm{H} & 1.03360 & 2.71266 & 1.93320\end{array}$

H $2.45622 \quad 4.49745 \quad 2.80991$
\end{abstract}

\section{Trans TS2 (B3LYP 6-31G*)}

$\mathrm{E}($ B3LYP 6-31G*) $=-1076.98745$

$\mathrm{E}\left(\right.$ B3LYP $\left.6-31 \mathrm{G}^{*}(\mathrm{THF})\right)=$ $-1076.99949$

$\begin{array}{cccc}\mathrm{O} & -.09009 & -.40568 & .24476 \\ \mathrm{C} & .05562 & -.03230 & 1.88404 \\ \mathrm{C} & 1.45984 & .02552 & 2.18790 \\ \mathrm{P} & 1.31770 & -.09476 & -.43413 \\ \mathrm{C} & -.78295 & 1.21359 & 2.05367 \\ \mathrm{C} & -1.92461 & 1.44199 & 1.26726 \\ \mathrm{C} & -2.72450 & 2.56354 & 1.47966 \\ \mathrm{C} & -2.40468 & 3.47767 & 2.48697 \\ \mathrm{C} & -1.27918 & 3.25755 & 3.27981 \\ \mathrm{C} & -.47644 & 2.13414 & 3.06594 \\ \mathrm{C} & 2.71258 & -1.27804 & -.34926 \\ \mathrm{C} & .76800 & -.28846 & -2.18469 \\ \mathrm{C} & 1.93832 & 1.63225 & -.40444 \\ \mathrm{H} & -.47151 & -.91999 & 2.24112 \\ \mathrm{H} & 1.87865 & 1.00811 & 2.39288 \\ \mathrm{H} & -2.17262 & .73584 & .48217 \\ \mathrm{H} & -3.60264 & 2.72333 & .85893 \\ \mathrm{H} & -3.02730 & 4.35373 & 2.64914 \\ \mathrm{H} & -1.02740 & 3.95286 & 4.07653 \\ \mathrm{H} & .37970 & 1.95189 & 3.70766 \\ \mathrm{H} & 3.10964 & -1.45886 & -1.35467 \\ \mathrm{H} & 2.35242 & -2.22081 & .07241 \\ \mathrm{H} & 3.49802 & -.90802 & .31139 \\ \mathrm{H} & 1.59870 & -.11506 & -2.87821\end{array}$




$\begin{array}{cccc}\mathrm{H} & -.03372 & .42265 & -2.40269 \\ \mathrm{H} & .38106 & -1.29948 & -2.34154 \\ \mathrm{H} & 2.17677 & 1.96624 & -1.42131 \\ \mathrm{H} & 2.82070 & 1.70990 & .23204 \\ \mathrm{H} & 1.15542 & 2.27644 & .00535 \\ \mathrm{C} & 3.72921 & -3.25504 & 3.81525 \\ \mathrm{C} & 4.22330 & -1.94670 & 3.83567 \\ \mathrm{C} & 3.47316 & -.89492 & 3.31941 \\ \mathrm{C} & 2.19039 & -1.09673 & 2.75182 \\ \mathrm{C} & 1.71626 & -2.43247 & 2.73273 \\ \mathrm{C} & 2.46735 & -3.48134 & 3.25998 \\ \mathrm{H} & 4.31384 & -4.07663 & 4.21964 \\ \mathrm{H} & 5.20174 & -1.74257 & 4.26594 \\ \mathrm{H} & 3.87161 & .11798 & 3.36150 \\ \mathrm{H} & .74530 & -2.65394 & 2.29450 \\ \mathrm{H} & 2.06076 & -4.49039 & 3.23072\end{array}$

\section{Cis stilbene (B3LYP 6-31G*)}

$\mathrm{E}($ B3LYP 6-31G*) $=-540.70134$

$\mathrm{E}(\mathrm{B} 3 \mathrm{LYP} 6-31 \mathrm{G} *(\mathrm{THF}))=$ $-540.84596$

$\begin{array}{lrrr}\text { C } & -1.45801 & 1.21407 & -0.78235 \\ \text { C } & -2.11440 & 0.42279 & 0.09204 \\ \text { C } & -0.03191 & 1.58696 & -0.86412 \\ \text { C } & 0.78306 & 1.76086 & 0.27014 \\ \text { C } & 2.11177 & 2.16014 & 0.13898 \\ \text { C } & 2.65904 & 2.39742 & -1.12564 \\ \text { C } & 1.85853 & 2.24633 & -2.26002 \\ \text { C } & 0.52627 & 1.85592 & -2.12776 \\ \text { H } & -2.07442 & 1.67765 & -1.55320 \\ \text { H } & -3.19901 & 0.39695 & -0.01748 \\ \text { H } & 0.36534 & 1.59347 & 1.25763 \\ \text { H } & 2.72136 & 2.29333 & 1.02929 \\ \text { H } & 3.69606 & 2.70783 & -1.22394 \\ \text { H } & 2.26926 & 2.43749 & -3.24828 \\ \text { H } & -0.09522 & 1.74820 & -3.01431 \\ \text { C } & -0.77709 & -2.16144 & 3.25469 \\ \text { C } & -1.99285 & -1.48074 & 3.34990 \\ \text { C } & -2.40683 & -0.64210 & 2.31543 \\ \text { C } & -1.60880 & -0.44646 & 1.17297 \\ \text { C } & -0.39477 & -1.15298 & 1.08462 \\ \text { C } & 0.01492 & -1.99842 & 2.11396 \\ \text { H } & -0.45399 & -2.82095 & 4.05601 \\ \text { H } & -2.62240 & -1.60661 & 4.22723 \\ \text { H } & -3.35903 & -0.12114 & 2.39109 \\ \text { H } & 0.22128 & -1.04517 & 0.19768 \\ \text { H } & 0.95391 & -2.53858 & 2.02181\end{array}$

\section{Trans stilbene (B3LYP 6-31G*)}

$\mathrm{E}\left(\mathrm{B} 3 \mathrm{LYP} 6-31 \mathrm{G}^{*}\right)=-540.70951$

$\mathrm{E}(\mathrm{B} 3 \mathrm{LYP} 6-31 \mathrm{G} *(\mathrm{THF}))=$ $-540.85466$

$\begin{array}{llll}\text { C } & -0.20524 & 0.07942 & 0.15132\end{array}$

$\begin{array}{llll}\text { C } & -1.05839 & -0.86576 & -0.29429\end{array}$

\begin{abstract}
$\begin{array}{llll}\text { C } & 1.26010 & 0.02140 & 0.14691\end{array}$
$\begin{array}{llll}\text { C } & 1.98019 & 1.09577 & 0.70372\end{array}$

$\begin{array}{llll}\text { C } & 3.37394 & 1.09759 & 0.73235\end{array}$

$\begin{array}{llll}\text { C } & 4.08602 & 0.02040 & 0.20153\end{array}$

$\begin{array}{llll}\text { C } & 3.38753 & -1.05534 & -0.35789\end{array}$

$\begin{array}{llll}\text { C } & 1.99623 & -1.05626 & -0.38580\end{array}$

$\begin{array}{llll}\mathrm{H} & -0.61357 & 0.99629 & 0.57418\end{array}$

H $-0.65014 \quad-1.78846-0.70432$

H $1.43180 \quad 1.93842 \quad 1.11983$

H $3.90315 \quad 1.94056 \quad 1.16948$

$\begin{array}{llll}\mathrm{H} & 5.17264 & 0.01722 & 0.22087\end{array}$

H $3.93243-1.89820-0.77575$

H $1.47740-1.90111-0.82966$

C $-5.35006-0.82056-0.31622$

$\begin{array}{llll}\text { C } & -4.63875 & -1.93148 & -0.77337\end{array}$

$\begin{array}{llll}\text { C } & -3.24484 & -1.92319 & -0.75806\end{array}$

$\begin{array}{llll}\text { C } & -2.52378 & -0.80930 & -0.28697\end{array}$

$\begin{array}{llll}\text { C } & -3.25905 & 0.30372 & 0.16883\end{array}$

$\begin{array}{llll}\text { C } & -4.65058 & 0.29636 & 0.15432\end{array}$

H $-6.43682-0.82185-0.32629$

H $-5.16874-2.80596-1.14229$

H $-2.69708-2.79283-1.11522$

H $-2.73930 \quad 1.18466 \quad 0.53459$

H $\quad-5.19489 \quad 1.16734 \quad 0.51081$
\end{abstract}

\section{E. $\mathrm{Ph}_{3} \mathrm{PCHPh}+$ PhCHO (reaction 4)}

\section{$\mathrm{Ph}_{3}$ PCHPh (B3LYP 6-31G*)} E(B3LYP 6-31G*) $=-1306.61226$
E(B3LYP 6-31G*(THF) $)=$
-1306.62585

$\begin{array}{lccc}\mathrm{C} & .43592 & .03863 & .43556 \\ \mathrm{P} & .45485 & -.17973 & 2.13267 \\ \mathrm{C} & 2.49018 & -2.05318 & -2.72385 \\ \mathrm{C} & 2.68448 & -2.43412 & -1.39113 \\ \mathrm{C} & 2.03829 & -1.77215 & -.34883 \\ \mathrm{C} & 1.15166 & -.68635 & -.58705 \\ \mathrm{C} & .97175 & -.32028 & -1.95104 \\ \mathrm{C} & 1.62280 & -.98407 & -2.98678 \\ \mathrm{H} & 2.99933 & -2.57140 & -3.53298 \\ \mathrm{H} & 3.35302 & -3.26088 & -1.15490 \\ \mathrm{H} & 2.22082 & -2.10685 & .66948 \\ \mathrm{H} & .30025 & .50535 & -2.18437 \\ \mathrm{H} & 1.45087 & -.66320 & -4.01347 \\ \mathrm{H} & -.18655 & .87221 & .12189 \\ \mathrm{C} & 4.33288 & 1.17124 & 4.32492 \\ \mathrm{C} & 3.13177 & 1.09625 & 5.03703 \\ \mathrm{C} & 1.96170 & .68098 & 4.39639 \\ \mathrm{C} & 1.98532 & .33384 & 3.03518 \\ \mathrm{C} & 3.19096 & .42612 & 2.32370 \\ \mathrm{C} & 4.36065 & .83677 & 2.96774 \\ \mathrm{H} & 5.24197 & 1.49801 & 4.82428 \\ \mathrm{H} & 3.10299 & 1.36805 & 6.08941 \\ \mathrm{H} & 1.03148 & .64065 & 4.95789\end{array}$

$\begin{array}{cccc}\mathrm{H} & 3.21307 & .18791 & 1.26425 \\ \mathrm{H} & 5.29018 & .90341 & 2.40734 \\ \mathrm{C} & -.45140 & -4.56059 & 3.38566 \\ \mathrm{C} & .35977 & -3.77495 & 4.20928 \\ \mathrm{C} & .66088 & -2.45903 & 3.84988 \\ \mathrm{C} & .14445 & -1.91609 & 2.66286 \\ \mathrm{C} & -.66680 & -2.71246 & 1.83843 \\ \mathrm{C} & -.96291 & -4.02820 & 2.19842 \\ \mathrm{H} & -.67909 & -5.58655 & 3.66539 \\ \mathrm{H} & .76390 & -4.18715 & 5.13058 \\ \mathrm{H} & 1.30143 & -1.86618 & 4.49460 \\ \mathrm{H} & -1.05208 & -2.29924 & .91053 \\ \mathrm{H} & -1.58703 & -4.63773 & 1.54939 \\ \mathrm{C} & -2.91377 & 2.55038 & 3.75617 \\ \mathrm{C} & -1.81132 & 3.09790 & 3.09113 \\ \mathrm{C} & -.78922 & 2.26766 & 2.62800 \\ \mathrm{C} & -.87060 & .87667 & 2.81675 \\ \mathrm{C} & -1.97487 & .33252 & 3.48746 \\ \mathrm{C} & -2.99231 & 1.16963 & 3.95546 \\ \mathrm{H} & -3.70769 & 3.19922 & 4.11860 \\ \mathrm{H} & -1.74416 & 4.17214 & 2.93626 \\ \mathrm{H} & .06579 & 2.70487 & 2.11851 \\ \mathrm{H} & -2.05030 & -.73996 & 3.64121 \\ \mathrm{H} & -3.84638 & .73986 & 4.47321\end{array}$

Ph $_{3}$ PCHPh
31G*(THF $)^{*} \quad$ (B3LYP 31G*(THF))

$\mathrm{E}($ B3LYP $6-31 \mathrm{G} *($ THF $))=$ $-1306.62856$

$\begin{array}{cccc}\mathrm{C} & .42806 & .04015 & .43246 \\ \mathrm{P} & .45117 & -.17774 & 2.12638 \\ \mathrm{C} & 2.48922 & -2.04756 & -2.72327 \\ \mathrm{C} & 2.67650 & -2.43378 & -1.39170 \\ \mathrm{C} & 2.02796 & -1.77317 & -.35005 \\ \mathrm{C} & 1.14657 & -.68433 & -.58981 \\ \mathrm{C} & .97315 & -.31329 & -1.95224 \\ \mathrm{C} & 1.62661 & -.97554 & -2.98684 \\ \mathrm{H} & 3.00019 & -2.56446 & -3.53188 \\ \mathrm{H} & 3.34112 & -3.26348 & -1.15571 \\ \mathrm{H} & 2.20471 & -2.11221 & .66811 \\ \mathrm{H} & .30552 & .51543 & -2.18487 \\ \mathrm{H} & 1.46050 & -.65084 & -4.01318 \\ \mathrm{H} & -.19665 & .87176 & .11963 \\ \mathrm{C} & 4.33846 & 1.15972 & 4.31077 \\ \mathrm{C} & 3.13668 & 1.10163 & 5.02320 \\ \mathrm{C} & 1.96363 & .69203 & 4.38508 \\ \mathrm{C} & 1.98417 & .33356 & 3.02709 \\ \mathrm{C} & 3.19002 & .41153 & 2.31422 \\ \mathrm{C} & 4.36299 & .81582 & 2.95597 \\ \mathrm{H} & 5.25061 & 1.48080 & 4.80808 \\ \mathrm{H} & 3.11016 & 1.38176 & 6.07354 \\ \mathrm{H} & 1.03217 & .66528 & 4.94523 \\ \mathrm{H} & 3.20862 & .16651 & 1.25618 \\ \mathrm{H} & 5.29304 & .86984 & 2.39518 \\ \mathrm{C} & -.44603 & -4.55663 & 3.39386 \\ \mathrm{C} & .32219 & -3.75127 & 4.23994\end{array}$




$\begin{array}{cccc}\mathrm{C} & .62134 & -2.43631 & 3.87689 \\ \mathrm{C} & .14453 & -1.91335 & 2.66454 \\ \mathrm{C} & -.62296 & -2.72913 & 1.81767 \\ \mathrm{C} & -.91611 & -4.04463 & 2.18121 \\ \mathrm{H} & -.67182 & -5.58217 & 3.67626 \\ \mathrm{H} & .69476 & -4.14794 & 5.18118 \\ \mathrm{H} & 1.22998 & -1.82726 & 4.53802 \\ \mathrm{H} & -.97487 & -2.32992 & .87065 \\ \mathrm{H} & -1.50525 & -4.66966 & 1.51457 \\ \mathrm{C} & -2.90968 & 2.54532 & 3.77462 \\ \mathrm{C} & -1.80656 & 3.09593 & 3.11406 \\ \mathrm{C} & -.78723 & 2.26755 & 2.64261 \\ \mathrm{C} & -.87166 & .87547 & 2.81921 \\ \mathrm{C} & -1.97750 & .32810 & 3.48447 \\ \mathrm{C} & -2.99222 & 1.16311 & 3.96047 \\ \mathrm{H} & -3.70135 & 3.19275 & 4.14384 \\ \mathrm{H} & -1.73671 & 4.17120 & 2.96897 \\ \mathrm{H} & .06870 & 2.70538 & 2.13564 \\ \mathrm{H} & -2.05525 & -.74568 & 3.62675 \\ \mathrm{H} & -3.84758 & .73094 & 4.47384\end{array}$

\section{Cis TS1 (B3L YP 6-31G*)}

$\mathrm{E}(\mathrm{B} 3 \mathrm{LYP}$ 6-31G*) $=-1652.16725$ $\mathrm{E}(\mathrm{B} 3 \mathrm{LYP} 6-31 \mathrm{G} *(\mathrm{THF}))=$ $-1652.1922$

$\begin{array}{lccc}\mathrm{O} & 1.62656 & .45993 & 2.24150 \\ \mathrm{C} & 1.68576 & .96817 & 1.05533 \\ \mathrm{P} & -.99647 & .20100 & -.05703 \\ \mathrm{C} & .80475 & -.02427 & -.16536 \\ \mathrm{C} & 3.08058 & 1.06256 & .42082 \\ \mathrm{C} & 1.25714 & -1.45280 & -.15706 \\ \mathrm{C} & -1.85873 & -.68465 & -1.40837 \\ \mathrm{C} & -1.74591 & -2.08651 & -1.47138 \\ \mathrm{C} & -2.35992 & -2.78945 & -2.50597 \\ \mathrm{C} & -3.08887 & -2.10959 & -3.48536 \\ \mathrm{C} & -3.20807 & -.72085 & -3.42420 \\ \mathrm{C} & -2.59757 & -.00806 & -2.39064 \\ \mathrm{C} & -1.31554 & 1.98607 & -.28310 \\ \mathrm{C} & -1.81675 & -.31898 & 1.48466 \\ \mathrm{C} & -. .74693 & 2.66320 & -1.37626 \\ \mathrm{C} & -.95643 & 4.03085 & -1.53983 \\ \mathrm{C} & -1.72976 & 4.73693 & -.61431 \\ \mathrm{C} & -2.29609 & 4.07004 & .47162 \\ \mathrm{C} & -2.09361 & 2.69860 & .63856 \\ \mathrm{C} & -1.18258 & -.12471 & 2.72377 \\ \mathrm{C} & -1.87020 & -.45621 & 3.89376 \\ \mathrm{C} & -3.16876 & -.96552 & 3.84081 \\ \mathrm{C} & -3.79728 & -1.14941 & 2.60660 \\ \mathrm{C} & -3.12729 & -.82720 & 1.42758 \\ \mathrm{C} & 4.12332 & .31043 & .96871 \\ \mathrm{C} & 5.40647 & .37664 & .42466 \\ \mathrm{C} & 5.66523 & 1.20575 & -.67019 \\ \mathrm{C} & 4.63202 & 1.97481 & -1.21263 \\ \mathrm{C} & 3.34930 & 1.90098 & -.66689 \\ \mathrm{C} & 1.21563 & -2.23113 & 1.01479 \\ \mathrm{C} & 1.67500 & -3.54977 & 1.00289\end{array}$

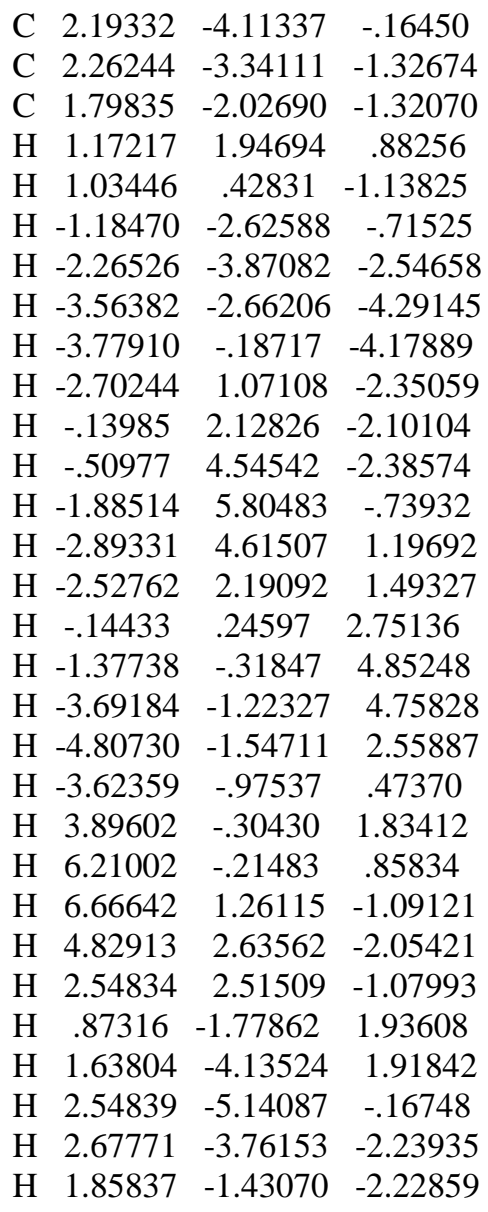

Cis TS1 (B3LYP 6-31G*(THF))

$\mathrm{E}\left(\mathrm{B} 3 \mathrm{LYP} 6-31 \mathrm{G}^{*}(\mathrm{THF})\right)=$
-1652.19158

$\begin{array}{lccc}\text { O } & 1.46625 & 1.11532 & 2.08385 \\ \text { C } & 1.68899 & 1.29779 & .84423 \\ \text { P } & -1.04521 & .18985 & -.14546 \\ \text { C } & .72317 & -.07751 & -.30038 \\ \text { C } & 3.09809 & 1.08788 & .32122 \\ \text { C } & 1.29651 & -1.42173 & -.02831 \\ \text { C } & -1.97833 & -.55797 & -1.53642 \\ \text { C } & -1.61285 & -1.83983 & -1.98399 \\ \text { C } & -2.30988 & -2.43930 & -3.03387 \\ \text { C } & -3.37139 & -1.76630 & -3.64694 \\ \text { C } & -3.74049 & -.49343 & -3.20394 \\ \text { C } & -3.04978 & .11069 & -2.15106 \\ \text { C } & -1.35018 & 1.99218 & -.25099 \\ \text { C } & -1.81435 & -.43176 & 1.38891 \\ \text { C } & -.89749 & 2.68991 & -1.38627 \\ \text { C } & -1.05398 & 4.07327 & -1.46760 \\ \text { C } & -1.66385 & 4.77295 & -.42088 \\ \text { C } & -2.12212 & 4.08394 & .70296 \\ \text { C } & -1.96700 & 2.69770 & .79075 \\ \text { C } & -1.27476 & -.06839 & 2.63686 \\ \text { C } & -1.89244 & -.50794 & 3.80857 \\ \text { C } & -3.03908 & -1.30584 & 3.74956\end{array}$

$\begin{array}{cccc}\text { C } & -3.57340 & -1.66709 & 2.51073 \\ \text { C } & -2.96779 & -1.23070 & 1.32991 \\ \text { C } & 4.02640 & .38644 & 1.09989 \\ \text { C } & 5.34030 & .21659 & .66192 \\ \text { C } & 5.74716 & .75424 & -.56318 \\ \text { C } & 4.82869 & 1.46100 & -1.34683 \\ \text { C } & 3.51374 & 1.62474 & -.90428 \\ \text { C } & 1.25093 & -2.02831 & 1.24446 \\ \text { C } & 1.89459 & -3.24310 & 1.48546 \\ \text { C } & 2.61295 & -3.88470 & .47381 \\ \text { C } & 2.68452 & -3.28943 & -.78896 \\ \text { C } & 2.03628 & -2.07962 & -1.03411 \\ \text { H } & 1.20484 & 2.14964 & .32657 \\ \text { H } & .96376 & .25886 & -1.31471 \\ \text { H } & -.78248 & -2.36421 & -1.52071 \\ \text { H } & -2.01982 & -3.42949 & -3.37569 \\ \text { H } & -3.90885 & -2.23293 & -4.46859 \\ \text { H } & -4.56554 & .03262 & -3.67732 \\ \text { H } & -3.34538 & 1.09951 & -1.81377 \\ \text { H } & -.42350 & 2.16097 & -2.20954 \\ \text { H } & -.69594 & 4.60449 & -2.34562 \\ \text { H } & -1.77965 & 5.85202 & -.48293 \\ \text { H } & -2.59762 & 4.62224 & 1.51879 \\ \text { H } & -2.31954 & 2.17709 & 1.67465 \\ \text { H } & -.36226 & .53042 & 2.67092 \\ \text { H } & -1.47125 & -.23008 & 4.77157 \\ \text { H } & -3.51275 & -1.64784 & 4.66668 \\ \text { H } & -4.46279 & -2.28984 & 2.45845 \\ \text { H } & -3.39524 & -1.52129 & .37616 \\ \text { H } & 3.69538 & -.00764 & 2.05510 \\ \text { H } & 6.05179 & -.32833 & 1.27921 \\ \text { H } & 6.77374 & .63075 & -.90143 \\ \text { H } & 5.14152 & 1.89152 & -2.29594 \\ \text { H } & 2.80279 & 2.18730 & -1.50917 \\ \text { H } & .73808 & -1.53166 & 2.05727 \\ \text { H } & 1.84237 & -3.68492 & 2.47863 \\ \text { H } & 3.11463 & -4.83008 & .66747 \\ \text { H } & 3.24799 & -3.76775 & -1.58764 \\ \text { H } & 2.10753 & -1.62222 & -2.01882\end{array}$

\section{Trans TS1 (B3LYP 6-31G*)}

$\mathrm{E}\left(\mathrm{B} 3 \mathrm{LYP} 6-31 \mathrm{G}^{*}\right)=-1652.16948$ $\mathrm{E}(\mathrm{B} 3 \mathrm{LYP} 6-31 \mathrm{G} *(\mathrm{THF}))=$ $-1652.19002$

$\begin{array}{lccc}\mathrm{O} & 1.16138 & 1.80992 & 1.45052 \\ \mathrm{C} & 1.77995 & .73128 & 1.16450 \\ \mathrm{P} & -.91922 & .20623 & -.05485 \\ \mathrm{C} & .80526 & -.27949 & -.21332 \\ \mathrm{C} & 3.14968 & .83939 & .51539 \\ \mathrm{C} & 1.11734 & -1.73056 & -.19624 \\ \mathrm{C} & -1.95555 & -.60495 & -1.34164 \\ \mathrm{C} & -1.92957 & -2.00557 & -1.48490 \\ \mathrm{C} & -2.67777 & -2.62397 & -2.48514 \\ \mathrm{C} & -3.45367 & -1.86031 & -3.36022 \\ \mathrm{C} & -3.47949 & -.47213 & -3.22952 \\ \mathrm{C} & -2.73646 & .15408 & -2.22757\end{array}$




$\begin{array}{cccc}\text { C } & -1.11429 & 1.99669 & -.33807 \\ \text { C } & -1.66806 & -.19260 & 1.56723 \\ \text { C } & -.23591 & 2.69589 & -1.17801 \\ \text { C } & -.45703 & 4.04421 & -1.45192 \\ \text { C } & -1.54990 & 4.70744 & -.89068 \\ \text { C } & -2.42532 & 4.01677 & -.05233 \\ \text { C } & -2.21126 & 2.66599 & .22285 \\ \text { C } & -1.11607 & .37830 & 2.72985 \\ \text { C } & -1.68657 & .09133 & 3.96972 \\ \text { C } & -2.79828 & -.74916 & 4.06481 \\ \text { C } & -3.35177 & -1.30478 & 2.91079 \\ \text { C } & -2.79199 & -1.02792 & 1.66278 \\ \text { C } & 4.05991 & -.22521 & .51045 \\ \text { C } & 5.31453 & -.08386 & -.08584 \\ \text { C } & 5.67662 & 1.12711 & -.67996 \\ \text { C } & 4.77991 & 2.19939 & -.66330 \\ \text { C } & 3.52873 & 2.05638 & -.06511 \\ \text { C } & .82576 & -2.55231 & .91414 \\ \text { C } & 1.19748 & -3.89691 & .93337 \\ \text { C } & 1.88775 & -4.45916 & -.14260 \\ \text { C } & 2.20359 & -3.65580 & -1.24107 \\ \text { C } & 1.82124 & -2.31639 & -1.26821 \\ \text { H } & 1.75537 & -.11167 & 1.89206 \\ \text { H } & 1.14437 & .17995 & -1.14579 \\ \text { H } & -1.32414 & -2.61307 & -.82144 \\ \text { H } & -2.64827 & -3.70559 & -2.58280 \\ \text { H } & -4.03235 & -2.34612 & -4.14112 \\ \text { H } & -4.07815 & .12997 & -3.90749 \\ \text { H } & -2.76466 & 1.23432 & -2.13938 \\ \text { H } & .63130 & 2.20476 & -1.60360 \\ \text { H } & .23478 & 4.57982 & -2.09576 \\ \text { H } & -1.71595 & 5.76036 & -1.10221 \\ \text { H } & -3.27332 & 4.52807 & .39459 \\ \text { H } & -2.89160 & 2.13977 & .88517 \\ \text { H } & -.25953 & 1.05091 & 2.62882 \\ \text { H } & -1.25873 & .53180 & 4.86622 \\ \text { H } & -3.23551 & -.96717 & 5.03578 \\ \text { H } & -4.22072 & -1.95366 & 2.97697 \\ \text { H } & -3.23348 & -1.46190 & .77257 \\ \text { H } & 3.78731 & -1.16623 & .98144 \\ \text { H } & 6.01248 & -.91789 & -.08078 \\ \text { H } & 6.65514 & 1.23861 & -1.14080 \\ \text { H } & 5.06427 & 3.15121 & -1.10685 \\ \text { H } & 2.82735 & 2.88336 & -.00578 \\ \text { H } & .31127 & -2.13266 & 1.77278 \\ \text { H } & .95538 & -4.50460 & 1.80196 \\ \text { H } & 2.18157 & -5.50506 & -.12215 \\ \text { H } & 2.74699 & -4.07487 & -2.08444 \\ \text { H } & 2.07253 & -1.70411 & -2.13060\end{array}$

Trans TS1 (B3LYP 6-31G*(THF))

$\mathrm{E}(\mathrm{B} 3 \mathrm{LYP} 6-31 \mathrm{G} *(\mathrm{THF}))=$ $-1652.19211$

$\begin{array}{llll}\mathrm{O} & 1.07655 & 1.87902 & 1.27755 \\ \mathrm{C} & 1.65436 & .75045 & 1.13253 \\ \mathrm{P} & -.96103 & .22299 & -.14669\end{array}$

\begin{tabular}{|c|c|c|c|}
\hline & & & \\
\hline & & & \\
\hline & & & \\
\hline & & & \\
\hline & & -1.61732 & \\
\hline & & & \\
\hline & & & \\
\hline & 609 & 60856 & \\
\hline & & -.04 & \\
\hline & & & \\
\hline & & & \\
\hline & & & \\
\hline & & & \\
\hline & & 4.7 & \\
\hline & & & \\
\hline & & & \\
\hline & & & \\
\hline & & & \\
\hline & & -.8 & \\
\hline & & $-1.2+x+3$ & \\
\hline & & & \\
\hline & & & \\
\hline & & & \\
\hline & & & \\
\hline & & & \\
\hline & & & \\
\hline & & & \\
\hline & & & \\
\hline & & & \\
\hline & & & \\
\hline & & & \\
\hline & & & \\
\hline & & & \\
\hline & & & \\
\hline & & & \\
\hline & & & \\
\hline & & & \\
\hline & & & \\
\hline & & & \\
\hline & & & \\
\hline & & & \\
\hline & & & \\
\hline & & & \\
\hline & & & \\
\hline & & & \\
\hline & & & \\
\hline & & & \\
\hline & & & \\
\hline & & & \\
\hline & & & \\
\hline & & & \\
\hline & & & \\
\hline & & & \\
\hline & & & \\
\hline & & & \\
\hline & & -5.34 & . \\
\hline & 3.2 & -3.8 & \\
\hline & 2.00 & -1.59100 & \\
\hline
\end{tabular}

\section{Cis OP1 (B3LYP 6-31G*)}

$\mathrm{E}(\mathrm{B} 3 \mathrm{LYP}$
$\mathrm{E}\left(\mathrm{B} 3 \mathrm{LY}-31 \mathrm{G}^{*}\right)=-1652.19287$
1652.2066

$\begin{array}{lllll}\mathrm{O} & .17117 & .54341 & -.04324\end{array}$

C $\quad .18686 \quad .15538 \quad 1.31666$

$\begin{array}{llll}\text { C } & 1.69860 & .37945 & 1.60402\end{array}$

$\begin{array}{lllll}\mathrm{P} & 1.99400 & .34971 & -.29585\end{array}$

$\begin{array}{llll}\text { C } & -.84421 & .87754 & 2.16208\end{array}$

$\begin{array}{llll}\text { C } & -.85094 & .72854 & 3.55569\end{array}$

C $-1.82263 \quad 1.35645 \quad 4.33315$

C $-2.80954 \quad 2.13815 \quad 3.72609$

$\begin{array}{llll}\text { C } & -2.81748 & 2.27931 & 2.33814\end{array}$

C $-1.84156 \quad 1.65081 \quad 1.56051$

$\begin{array}{llll}\mathrm{H} & -.01449 & -.92785 & 1.41301\end{array}$

$\begin{array}{llll}\mathrm{H} & 2.19911 & -.48690 & 2.05242\end{array}$

$\begin{array}{lllll}\mathrm{H} & -.08451 & .12494 & 4.03760\end{array}$

$\mathrm{H}-1.80816 \quad 1.23895 \quad 5.41385$

H -3.56698 $2.62910 \quad 4.33179$

H $-3.586852 .87783 \quad 1.85622$

$\begin{array}{llll}\mathrm{H} & -1.84849 & 1.74266 & .47941\end{array}$

C $3.03295 \quad 3.90135 \quad 3.73190$

$\begin{array}{lll}\text { C } 3.54129 & 2.64195 & 4.04937\end{array}$

C $3.09686 \quad 1.51397 \quad 3.35739$

$\begin{array}{llll}\text { C } & 2.13925 & 1.61943 & 2.33944\end{array}$

$\begin{array}{llll}\text { C } & 1.63103 & 2.89349 & 2.03317\end{array}$

C $2.07514 \quad 4.02093 \quad 2.72114$

H $3.37703 \quad 4.78252 \quad 4.26703$

$\begin{array}{llll}\mathrm{H} & 4.28538 & 2.53361 & 4.83429\end{array}$

$\begin{array}{llll}\mathrm{H} & 3.50415 & .53635 & 3.60494\end{array}$

$\begin{array}{llll}\mathrm{H} & .88108 & 3.00170 & 1.25677\end{array}$

$\begin{array}{llll}\mathrm{H} & 1.66870 & 4.99690 & 2.46884\end{array}$

$\begin{array}{lllll}\text { C } & 1.86685 & 3.57679 & -3.68277\end{array}$

$\begin{array}{llll}\text { C } & .86485 & 3.54980 & -2.71305\end{array}$

$\begin{array}{llll}\text { C } & .89057 & 2.59138 & -1.69779\end{array}$

C $1.93931 \quad 1.66194-1.61897$

$\begin{array}{llll}\text { C } & 2.94649 & 1.70165 & -2.60029\end{array}$

$\begin{array}{llll}\text { C } & 2.90162 & 2.64037 & -3.63043\end{array}$

H $1.84201 \quad 4.31972 \quad-4.47589$

$\mathrm{H} \quad .05252 \quad 4.27118$-2.74692

$\begin{array}{llll}\mathrm{H} & .09210 & 2.54512 & -.96983\end{array}$

H $3.76990 \quad .99686-2.56396$

H $3.68304 \quad 2.64496-4.38573$

$\begin{array}{llll}\text { C } & 6.72388 & .41142 & .18583\end{array}$

$\begin{array}{llll}\text { C } & 5.95645 & 1.56712 & .35030\end{array}$

$\begin{array}{llll}\text { C } & 4.57465 & 1.52240 & .16912\end{array}$

$\begin{array}{llll}\text { C } & 3.91548 & .32640 & -.17476\end{array}$

$\begin{array}{llll}\text { C } & 4.70504 & -.82160 & -.33982\end{array}$

$\begin{array}{llll}\text { C } & 6.09275 & -.78122 & -.16309\end{array}$

$\begin{array}{llll}\mathrm{H} & 7.80182 & .44382 & .32273\end{array}$

$\begin{array}{llll}\text { H } & 6.43339 & 2.50711 & .61699\end{array}$

$\begin{array}{lllll}\mathrm{H} & 4.00567 & 2.44091 & .28463\end{array}$

$\begin{array}{lllll}\mathrm{H} & 4.24798 & -1.76353 & -.62398\end{array}$

$\begin{array}{llll}\mathrm{H} & 6.67760 & -1.68681 & -.30673\end{array}$

C $1.15479-3.86730-2.06561$ 


\begin{abstract}
$\begin{array}{llll}\text { C } & 1.01015 & -2.72547 & -2.85629\end{array}$
C $1.28428-1.46345-2.32965$

$\begin{array}{llll}\text { C } & 1.72282 & -1.32691 & -1.00482\end{array}$

$\begin{array}{llll}\text { C } & 1.86486 & -2.47771 & -.21568\end{array}$

$\begin{array}{llll}\text { C } & 1.57930 & -3.73932 & -.74291\end{array}$

H $\quad .93582-4.84902-2.47675$

H $\quad .67571 \quad-2.81408$-3.88662

$\begin{array}{lllll}\mathrm{H} & 1.14769 & -.58308 & -2.94995\end{array}$

H 2.21008 -2.40181 $\quad .81188$

H $1.69261 \quad-4.62037 \quad-.11671$
\end{abstract}

\section{Trans OP1 (B3LYP 6-31G*)}

$\mathrm{E}\left(\mathrm{B} 3 \mathrm{LYP} 6-31 \mathrm{G}^{*}\right)=-1652.19841$

$\mathrm{E}(\mathrm{B} 3 \mathrm{LYP}$ 6-31G*(THF) $)=$ $-1652.21133$

$\begin{array}{cccc}\mathrm{O} & 1.30231 & .12241 & 3.66791 \\ \mathrm{C} & .53420 & -.90197 & 3.06563 \\ \mathrm{C} & -.38328 & -.02655 & 2.19356 \\ \mathrm{P} & .73092 & 1.50376 & 2.59033 \\ \mathrm{H} & -1.32025 & .18185 & 2.72566 \\ \mathrm{C} & -1.30033 & -1.19493 & -1.86043 \\ \mathrm{C} & -2.31136 & -.75362 & -1.00591 \\ \mathrm{C} & -2.00664 & -.37725 & .30236 \\ \mathrm{C} & -.69009 & -.43109 & .78220 \\ \mathrm{C} & .31824 & -.87813 & -.08877 \\ \mathrm{C} & .01613 & -1.25550 & -1.39604 \\ \mathrm{H} & -1.53473 & -1.49071 & -2.87957 \\ \mathrm{H} & -3.33908 & -.70194 & -1.35574 \\ \mathrm{H} & -2.79757 & -.02869 & .96219 \\ \mathrm{H} & 1.34871 & -.92217 & .25422 \\ \mathrm{H} & .81079 & -1.59763 & -2.05398 \\ \mathrm{C} & 4.97269 & 2.02136 & .68708 \\ \mathrm{C} & 4.65344 & .87275 & 1.41124 \\ \mathrm{C} & 3.38216 & .71883 & 1.96603 \\ \mathrm{C} & 2.39370 & 1.70053 & 1.78312 \\ \mathrm{C} & 2.73064 & 2.85507 & 1.05502 \\ \mathrm{C} & 4.00894 & 3.01664 & .52020 \\ \mathrm{H} & 5.96598 & 2.14447 & .26321 \\ \mathrm{H} & 5.39876 & .09580 & 1.56010 \\ \mathrm{H} & 3.15503 & -.14502 & 2.57739 \\ \mathrm{H} & 1.99568 & 3.63494 & .89508 \\ \mathrm{H} & 4.24707 & 3.92240 & -.03118 \\ \mathrm{C} & -2.15023 & 4.07295 & -.17901 \\ \mathrm{C} & -1.09398 & 3.35954 & -.74727 \\ \mathrm{C} & -.19813 & 2.66195 & .06489 \\ \mathrm{C} & -.31351 & 2.67262 & 1.46725 \\ \mathrm{C} & -1.39768 & 3.37992 & 2.01495 \\ \mathrm{C} & -2.30349 & 4.07268 & 1.20717 \\ \mathrm{H} & -2.84912 & 4.61612 & -.80990 \\ \mathrm{H} & -.96777 & 3.33718 & -1.82688 \\ \mathrm{H} & .60191 & 2.10351 & -.41167 \\ \mathrm{H} & -1.54292 & 3.40250 & 3.09114 \\ \mathrm{H} & -3.12592 & 4.61577 & 1.66659 \\ \mathrm{C} & .50152 & 4.24183 & 6.36697 \\ \mathrm{C} & 1.00418 & 4.73824 & 5.16209 \\ \mathrm{C} & 1.05189 & 3.92025 & 4.03381 \\ & & & \end{array}$

$\begin{array}{cccc}\mathrm{C} & .63082 & 2.58378 & 4.10223 \\ \mathrm{C} & .14345 & 2.08896 & 5.31843 \\ \mathrm{C} & .06419 & 2.91918 & 6.43865 \\ \mathrm{H} & .45434 & 4.88229 & 7.24394 \\ \mathrm{H} & 1.35396 & 5.76531 & 5.09709 \\ \mathrm{H} & 1.42542 & 4.32887 & 3.10009 \\ \mathrm{H} & -.15917 & 1.05138 & 5.39441 \\ \mathrm{H} & -.32829 & 2.52340 & 7.37183 \\ \mathrm{C} & -1.20332 & -2.64601 & 3.66973 \\ \mathrm{C} & -.16716 & -1.79304 & 4.07446 \\ \mathrm{C} & .23182 & -1.79730 & 5.41519 \\ \mathrm{C} & -.40097 & -2.63292 & 6.33871 \\ \mathrm{C} & -1.43641 & -3.47528 & 5.93138 \\ \mathrm{C} & -1.83490 & -3.48030 & 4.59202 \\ \mathrm{H} & -1.51880 & -2.65462 & 2.62839 \\ \mathrm{H} & 1.03956 & -1.13941 & 5.71980 \\ \mathrm{H} & -.08259 & -2.62535 & 7.37837 \\ \mathrm{H} & -1.92962 & -4.12432 & 6.65044 \\ \mathrm{H} & -2.64089 & -4.13300 & 4.26585 \\ \mathrm{H} & 1.16415 & -1.54220 & 2.42349\end{array}$

\section{Cis OP2 (B3LYP 6-31G*)}

$\mathrm{E}\left(\mathrm{B} 3 \mathrm{LYP} 6-31 \mathrm{G}^{*}\right)=-1652.18935$

$\mathrm{E}(\mathrm{B} 3 \mathrm{LYP} 6-31 \mathrm{G} *(\mathrm{THF}))=$ $-1652.21005$

$\begin{array}{llllllll}\mathrm{O} & .31169 & .20304 & -.04957 & \text { H } & 2.30330 & -2.38120 & -1.74683 \\ \mathrm{C} & .09359 & .28172 & 1.37745 & \text { H } & 4.10871 & -.67720 & 1.77338 \\ \mathrm{C} & 1.55571 & .15271 & 1.84845 & \text { H } & 5.57054 & -2.64226 & 1.97496\end{array}$

$\begin{array}{llll}\text { C } & -.73962 & 1.48868 & 1.75459\end{array}$

C $-1.09364 \quad 1.68547 \quad 3.09751$

C $-1.89224 \quad 2.76423 \quad 3.47173$

C $-2.35484 \quad 3.66322 \quad 2.50673$

C $-2.02013 \quad 3.46517 \quad 1.16730$

$\begin{array}{llll}\text { C }-1.22120 & 2.38164 & .79216\end{array}$

$\begin{array}{llll}\mathrm{H} & -.47128 & -.61510 & 1.66535\end{array}$

$\begin{array}{llll}\mathrm{H} & 1.71188 & -.87733 & 2.18082\end{array}$

$\begin{array}{llll}\mathrm{H} & -.73299 & .99340 & 3.85488\end{array}$

H $-2.15093 \quad 2.90561 \quad 4.51797$

H $-2.97661 \quad 4.505772 .79807$

H $-2.38578 \quad 4.15122 \quad .40726$

$\begin{array}{lllll}\mathrm{H} & -.98103 & 2.21365 & -.25224\end{array}$

C $3.40844 \quad 2.68743 \quad 4.82733$

C $3.48800 \quad 1.29672 \quad 4.91390$

$\begin{array}{llll}\text { C } & 2.86917 & .50102 & 3.94981\end{array}$

$\begin{array}{llll}\text { C } & 2.16417 & 1.06309 & 2.86769\end{array}$

$\begin{array}{llll}\text { C } & 2.09609 & 2.46817 & 2.80050\end{array}$

$\begin{array}{llll}\text { C } & 2.70732 & 3.26526 & 3.76697\end{array}$

H $3.88456 \quad 3.31354 \quad 5.57744$

$\begin{array}{lllll}\mathrm{H} & 4.02292 & .82858 & 5.73670\end{array}$

$\begin{array}{llll}\mathrm{H} & 2.92319 & -.58268 & 4.03688\end{array}$

H $1.56468 \quad 2.94451 \quad 1.98483$

H $2.63462 \quad 4.34703 \quad 3.68814$

$\begin{array}{llll}\text { C } & 1.63605 & -.14260 & -4.89494\end{array}$

$\begin{array}{llll}\text { C } & .50656 & -.36629 & -4.10905\end{array}$

$\begin{array}{llll}\text { C } & .58636 & -.28642 & -2.71438\end{array}$

\section{Trans OP2 (B3LYP 6-31G*)}

\begin{abstract}
$\begin{array}{llll}\text { C } & 1.79727 & .02304 & -2.07428\end{array}$
$\begin{array}{llll}\text { C } & 2.92603 & .23816 & -2.88487\end{array}$

$\begin{array}{llll}\text { C } & 2.85114 & .15782 & -4.27496\end{array}$

$\begin{array}{lllll}\mathrm{H} & 1.57204 & -.20004 & -5.97861\end{array}$

$\mathrm{H} \quad-.44621 \quad-.60113 \quad-4.57766$

$\begin{array}{lllll}\mathrm{H} & -.30204 & -.45927 & -2.12018\end{array}$

$\begin{array}{llll}\mathrm{H} & 3.88289 & .48061 & -2.42862\end{array}$

$\begin{array}{llll}\text { H } & 3.74127 & .33500 & -4.87368\end{array}$

$\begin{array}{llll}\text { C } & 4.29459 & 4.20384 & -.30412\end{array}$

$\begin{array}{llll}\text { C } & 4.96178 & 3.06983 & .15845\end{array}$

$\begin{array}{llll}\text { C } & 4.31008 & 1.83522 & .20203\end{array}$

$\begin{array}{llll}\text { C } & 2.98500 & 1.71715 & -.23559\end{array}$

$\begin{array}{llll}\text { C } & 2.32677 & 2.85935 & -.71683\end{array}$

$\begin{array}{llll}\text { C } & 2.97245 & 4.09501 & -.74026\end{array}$

$\begin{array}{llll}\mathrm{H} & 4.80151 & 5.16473 & -.32877\end{array}$

$\begin{array}{lllll}\mathrm{H} & 5.99318 & 3.14148 & .49368\end{array}$

$\begin{array}{llll}\mathrm{H} & 4.84853 & .96953 & .57133\end{array}$

H $1.305552 .78423 \quad-1.07912$

H $\quad 2.44408 \quad 4.97025-1.10905$

$\begin{array}{llll}\text { C } & 4.73357 & -3.65024 & .25906\end{array}$

$\begin{array}{llll}\text { C } 3.79931 & -3.57310 & -.77297\end{array}$

$\begin{array}{llll}\text { C } 3.00936 & -2.43109 & -.92562\end{array}$

$\begin{array}{llll}\text { C } 3.12304 & -1.35648 & -.03113\end{array}$

C $4.03736-1.46608 \quad 1.03216$

C $4.85255-2.58977 \quad 1.16064$

$\begin{array}{llll}\text { H } & 5.35843 & -4.53275 & .36766\end{array}$

H $3.68641-4.39859-1.47082$

H $2.30330 \quad-2.38120 \quad-1.74683$

$\begin{array}{llll}H & 4.10871 & -.67720 & 1.77338\end{array}$
\end{abstract}

$\mathrm{E}\left(\mathrm{B} 3 \mathrm{LYP} 6-31 \mathrm{G}^{*}\right)=-1652.19723$

$\mathrm{E}\left(\right.$ B3LYP $\left.6-31 \mathrm{G}^{*}(\mathrm{THF})\right)=$ $-1652.21005$

$\begin{array}{cccc}\mathrm{O} & .35535 & .45111 & -.11636 \\ \mathrm{C} & .20344 & .35694 & 1.29652 \\ \mathrm{C} & 1.55784 & -.29584 & 1.61627 \\ \mathrm{P} & 2.12455 & .13901 & -.22021 \\ \mathrm{H} & .14126 & 1.35731 & 1.74992 \\ \mathrm{H} & 1.44217 & -1.38681 & 1.61332 \\ \mathrm{C} & 3.76078 & .84967 & 5.14857 \\ \mathrm{C} & 3.58290 & -.49723 & 4.82929 \\ \mathrm{C} & 2.87109 & -.85634 & 3.68480 \\ \mathrm{C} & 2.32384 & .11711 & 2.83452 \\ \mathrm{C} & 2.50892 & 1.47025 & 3.17053 \\ \mathrm{C} & 3.21999 & 1.83132 & 4.31387 \\ \mathrm{H} & 4.31309 & 1.13331 & 6.04059 \\ \mathrm{H} & 3.99580 & -1.27072 & 5.47171 \\ \mathrm{H} & 2.73533 & -1.90735 & 3.44146 \\ \mathrm{H} & 2.10507 & 2.24678 & 2.52740 \\ \mathrm{H} & 3.35197 & 2.88324 & 4.55401 \\ \mathrm{C} & 1.55594 & -.90850 & -4.76801 \\ \mathrm{C} & .48882 & -1.08435 & -3.88850 \\ \mathrm{C} & .63504 & -.78716 & -2.53033 \\ \mathrm{C} & 1.86024 & -.32859 & -2.02822\end{array}$




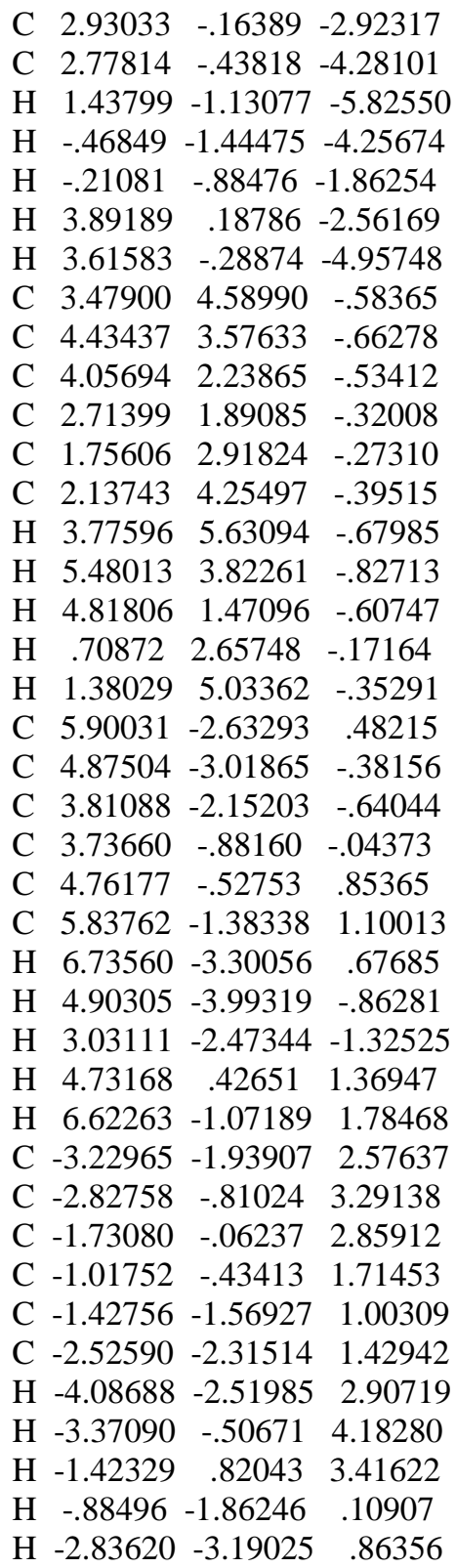

\section{Cis TS2 (B3LYP 6-31G*)}

$\mathrm{E}\left(\mathrm{B} 3 \mathrm{LYP} 6-31 \mathrm{G}^{*}\right)=-1652.17317$

$\mathrm{E}(\mathrm{B} 3 \mathrm{LYP} 6-31 \mathrm{G} *(\mathrm{THF}))=$ $-1652.18622$

$\begin{array}{cccc}\mathrm{O} & .63360 & .39427 & -.36160 \\ \mathrm{C} & .37804 & .24424 & 1.27776 \\ \mathrm{C} & 1.65299 & .23867 & 1.95924 \\ \mathrm{P} & 2.17063 & .21291 & -.73027 \\ \mathrm{C} & -.70508 & 1.25658 & 1.58433 \\ \mathrm{C} & -1.11332 & 1.37307 & 2.92243 \\ \mathrm{C} & -2.14891 & 2.23405 & 3.27981 \\ \mathrm{C} & -2.79234 & 3.00260 & 2.30662 \\ \mathrm{C} & -2.39509 & 2.89209 & .97455 \\ \mathrm{C} & -1.36417 & 2.02008 & .61313 \\ \mathrm{H} & -.05810 & -.75683 & 1.24153\end{array}$

$\begin{array}{cccc}\mathrm{H} & 1.99113 & -.75749 & 2.23160 \\ \mathrm{H} & -.60090 & .79221 & 3.68418 \\ \mathrm{H} & -2.44601 & 2.31292 & 4.32245 \\ \mathrm{H} & -3.59496 & 3.68039 & 2.58585 \\ \mathrm{H} & -2.89021 & 3.48245 & .20724 \\ \mathrm{H} & -1.06905 & 1.92938 & -.42569 \\ \mathrm{C} & 3.60695 & 3.24711 & 4.34191 \\ \mathrm{C} & 3.95452 & 1.89493 & 4.42738 \\ \mathrm{C} & 3.29419 & .94593 & 3.65520 \\ \mathrm{C} & 2.25724 & 1.29520 & 2.75083 \\ \mathrm{C} & 1.92820 & 2.67244 & 2.68022 \\ \mathrm{C} & 2.58638 & 3.61789 & 3.46377 \\ \mathrm{H} & 4.11527 & 3.99106 & 4.94956 \\ \mathrm{H} & 4.73982 & 1.57618 & 5.11010 \\ \mathrm{H} & 3.56226 & -.10506 & 3.75670 \\ \mathrm{H} & 1.14237 & 3.00539 & 2.01220 \\ \mathrm{H} & 2.29580 & 4.66354 & 3.38211 \\ \mathrm{C} & 1.74583 & .13830 & -5.36522 \\ \mathrm{C} & .61735 & -.04121 & -4.56435 \\ \mathrm{C} & .73075 & -.01602 & -3.17238 \\ \mathrm{C} & 1.97972 & .18893 & -2.56728 \\ \mathrm{C} & 3.10899 & .37024 & -3.38128 \\ \mathrm{C} & 2.99287 & .34429 & -4.77031 \\ \mathrm{H} & 1.65475 & .12316 & -6.44806 \\ \mathrm{H} & -. .35687 & -.19590 & -5.02051 \\ \mathrm{H} & -.14632 & -.14386 & -2.54827 \\ \mathrm{H} & 4.08254 & .54702 & -2.93202 \\ \mathrm{H} & 3.87515 & .49086 & -5.38750 \\ \mathrm{C} & 4.79519 & 3.96957 & -.24508 \\ \mathrm{C} & 5.40703 & 2.71426 & -.22252 \\ \mathrm{C} & 4.64290 & 1.55386 & -.33598 \\ \mathrm{C} & 3.24647 & 1.64175 & -.46023 \\ \mathrm{C} & 2.63492 & 2.90642 & -.47804 \\ \mathrm{C} & 3.40921 & 4.06066 & -.37711 \\ \mathrm{H} & 5.39568 & 4.87049 & -.15561 \\ \mathrm{H} & 6.48603 & 2.63517 & -.12115 \\ \mathrm{H} & 5.13766 & .58835 & -.33414 \\ \mathrm{H} & 1.55587 & 2.98522 & -.57186 \\ \mathrm{H} & 2.92406 & 5.03278 & -.38872 \\ \mathrm{C} & 4.22053 & -3.86058 & .09372 \\ \mathrm{C} & 3.28488 & -3.72466 & -.93090 \\ \mathrm{C} & 2.69078 & -2.48582 & -1.18069 \\ \mathrm{C} & 3.00770 & -1.37381 & -.38282 \\ \mathrm{C} & 3.93271 & -1.52546 & .66595 \\ \mathrm{C} & 4.54644 & -2.75507 & .88687 \\ \mathrm{H} & 4.68967 & -4.82264 & .28070 \\ \mathrm{H} & 3.01992 & -4.57940 & -1.54716 \\ \mathrm{H} & 1.98271 & -2.38971 & -1.99785 \\ \mathrm{H} & 4.14641 & -.68988 & 1.32313 \\ \mathrm{H} & 5.26667 & -2.85588 & 1.69413 \\ & & & \end{array}$

Trans TS2 (B3LYP 6-31G*)

$\mathrm{E}(\mathrm{B} 3 \mathrm{LYP}$ 6-31G*) $=-1652.17868$

$\mathrm{E}(\mathrm{B} 3 \mathrm{LYP}$ 6-31G*(THF) $)=$ $-1652.19331$

$\begin{array}{llll}\text { O } & .64803 & .38205 & -.27252\end{array}$

\begin{tabular}{|c|c|c|c|}
\hline & & & \\
\hline & 188 & & \\
\hline & & & \\
\hline & 677 & 6699 & \\
\hline & 2.24241 & -.89194 & \\
\hline & & 3.20068 & \\
\hline & & & \\
\hline & & & \\
\hline & & & \\
\hline & & & \\
\hline & & & \\
\hline & & & \\
\hline & & & \\
\hline & & & \\
\hline & & & \\
\hline & & & \\
\hline & & & \\
\hline & & & \\
\hline & & & \\
\hline & & & \\
\hline & & & \\
\hline & & & \\
\hline & & & \\
\hline & & & \\
\hline & & & \\
\hline & & & \\
\hline & & & \\
\hline & & & \\
\hline & & & \\
\hline & & & \\
\hline & & & \\
\hline & & & \\
\hline & & & \\
\hline & & & \\
\hline & & & \\
\hline & & & \\
\hline & & & \\
\hline & & & \\
\hline & & & \\
\hline & & & \\
\hline & & & \\
\hline & & & \\
\hline & & & \\
\hline & & & \\
\hline & & & \\
\hline & & & \\
\hline & & & \\
\hline & & & \\
\hline & & & \\
\hline & & & \\
\hline & & & \\
\hline & & -.8 & \\
\hline & & & \\
\hline & & & \\
\hline & & & \\
\hline & & & \\
\hline & & & \\
\hline & -2.0 & .15/ & $1.00 J$ \\
\hline
\end{tabular}




\section{H $1.16714 \quad-2.52929 \quad 1.09862$ \\ H $\quad-.35976 \quad-4.44955 \quad 1.31698$ \\ F. $(\mathrm{MeO})_{3} \mathrm{PCHPh}+$ PhCHO (reaction 5)}

\section{$(\mathrm{MeO})_{3}$ PCHPh (B3LYP 6-31G*)}

\begin{tabular}{|c|c|c|c|}
\hline & 0.6 & 589 & \\
\hline & -0.05435 & 1.15317 & \\
\hline & -1.21301 & 1.78805 & \\
\hline$)$ & -0.6 & & \\
\hline & -0.6 & & \\
\hline$)$ & 1.6 & & \\
\hline & $2.8^{\prime}$ & 6 & \\
\hline & 0.6 & 0.6 & \\
\hline & -1.02345 & 2.08218 & \\
\hline & -2.0 & 1.1 & \\
\hline & -1. & 2.6 & \\
\hline & 4 & -2.4 & \\
\hline & 0 & & \\
\hline & & & \\
\hline & 3.6 & & \\
\hline & 3.12638 & 0.1 & \\
\hline & 2.82294 & 1.08189 & \\
\hline & 1.28 & -3.5 & \\
\hline & 1.3 & -2.2 & \\
\hline & 1.0 & -1.2 & \\
\hline & 0.8 & -1.4 & \\
\hline & & & \\
\hline & 1.0 & & \\
\hline & $1.45 \mathrm{~s}$ & -4.3 & \\
\hline & 1.51836 & -2.00 & 4.25 \\
\hline & 112 & & \\
\hline & 0.581 & -3.05511 & -0.4 \\
\hline & 0.98904 & -4.87236 & 1.09 \\
\hline
\end{tabular}

(MeO) $)_{3}$ PCHPh

(B3LYP 31G*(THF))

\footnotetext{
$\mathrm{E}(\mathrm{B} 3 \mathrm{LYP} 6-31 \mathrm{G} *(\mathrm{THF}))=$ $-1306.62856$
}

$\begin{array}{cccl}\mathrm{H} & .69392 & .66648 & .46770 \\ \mathrm{H} & -1.06541 & 2.06879 & -.50955 \\ \mathrm{H} & -2.10244 & 1.08549 & -1.58640 \\ \mathrm{H} & -1.45050 & 2.64758 & -2.15730 \\ \mathrm{H} & -1.61484 & -2.46413 & -3.57729 \\ \mathrm{H} & .15708 & -2.38228 & -3.82336 \\ \mathrm{H} & -.86183 & -.95069 & -4.15852 \\ \mathrm{H} & 3.62889 & -.45103 & -3.05442 \\ \mathrm{H} & 3.18167 & .03900 & -1.39113 \\ \mathrm{H} & 2.83489 & 1.12803 & -2.77185 \\ \mathrm{C} & 1.25820 & -3.57249 & 2.81026 \\ \mathrm{C} & 1.30612 & -2.23627 & 3.22135 \\ \mathrm{C} & 1.10941 & -1.20275 & 2.30728 \\ \mathrm{C} & .85337 & -1.45814 & .93792 \\ \mathrm{C} & .80847 & -2.81726 & .54294 \\ \mathrm{C} & 1.00752 & -3.84713 & 1.46130 \\ \mathrm{H} & 1.40992 & -4.37948 & 3.52319 \\ \mathrm{H} & 1.49828 & -1.99380 & 4.26524 \\ \mathrm{H} & 1.14956 & -.17006 & 2.65023 \\ \mathrm{H} & .60038 & -3.06972 & -.49355 \\ \mathrm{H} & .96111 & -4.87923 & 1.11777\end{array}$

\section{Cis TS1 (B3LYP 6-31G*)}

$\mathrm{E}(\mathrm{B} 3 \mathrm{LYP}$ 6-31G*) $=-1302.65534$ $\mathrm{E}(\mathrm{B} 3 \mathrm{LYP} 6-31 \mathrm{G} *(\mathrm{THF}))=$ $-1302.67268$

$$
\begin{array}{llll}
\mathrm{O} & -. .33657 & 1.23944 & -.24335 \\
\mathrm{C} & -.17413 & .56580 & .83110 \\
\mathrm{C} & 1.77459 & .34515 & 1.23789 \\
\mathrm{P} & 2.43786 & -.20744 & -.28162 \\
\mathrm{C} & -.69126 & 1.12106 & 2.13695 \\
\mathrm{C} & -. .78907 & .31945 & 3.28018 \\
\mathrm{C} & -1.28928 & .84137 & 4.47214 \\
\mathrm{C} & -1.70274 & 2.17567 & 4.52995 \\
\mathrm{C} & -1.61954 & 2.97666 & 3.38892 \\
\mathrm{C} & -1.11821 & 2.45087 & 2.19819 \\
\mathrm{O} & 2.81193 & .90351 & -1.35290 \\
\mathrm{C} & 1.95239 & 1.81785 & -2.09191 \\
\mathrm{O} & 3.84070 & -.93157 & -.03565 \\
\mathrm{C} & 4.59562 & -1.52195 & -1.11753 \\
\mathrm{O} & 1.58510 & -1.37701 & -.95462 \\
\mathrm{C} & .43572 & -1.22428 & -1.83560
\end{array}
$$$$
\begin{array}{llllll}
\text { 6- } & \mathrm{H} & -.32991 & -.53433 & .79382
\end{array}
$$$$
\text { H } 1.80509-51.51666 \quad 1.91231
$$$$
\begin{array}{llll}
\mathrm{H} & -.47562 & -.72282 & 3.23196
\end{array}
$$$$
\text { H }-1.36203 \quad 20956 \quad 5.35405
$$$$
\text { H }-2.09390 \quad 2.58500 \quad 5.45801
$$$$
\text { H }-1.94995 \quad 4.012003 .42709
$$$$
\text { H }-1.064043 .04646 \quad 1.29245
$$$$
\text { H } 1.825191 .41370-3.09976
$$$$
\text { H } 2.49687 \quad 2.76205-2.13814
$$$$
\text { H } \quad .99125 \quad 1.92700 \quad-1.58528
$$$$
\text { H } 4.00088 \text { - } 2.29027-1.61837
$$$$
\text { H } 4.89708 \text {-.74922 }-1.82916
$$$$
\text { H } 5.47634 \quad-1.97074 \quad-.65734
$$$$
\text { H } \quad-.12741 \quad-2.15393 \quad-1.74567
$$

\begin{abstract}
H $\quad .79967-1.11513 \quad-2.86178$
$\begin{array}{llll}\mathrm{H} & -.16293 & -.35912 & -1.52913\end{array}$

C $3.62931 \quad 3.824562 .99186$

$\begin{array}{llll}\text { C } 3.76200 & 2.57089 & 3.59262\end{array}$

C $3.17292 \quad 1.44827 \quad 3.01363$

$\begin{array}{llll}\text { C } & 2.43259 & 1.55037 & 1.82323\end{array}$

$\begin{array}{llll}\text { C } & 2.28779 & 2.82279 & 1.24083\end{array}$

C $2.88504 \quad 3.94318 \quad 1.81682$

H $4.09409 \quad 4.69946 \quad 3.43861$

H $4.330812 .46401 \quad 4.51296$

$\begin{array}{llll}\mathrm{H} & 3.28941 & .47458 & 3.48405\end{array}$

$\begin{array}{llll}\mathrm{H} & 1.66749 & 2.93417 & .35830\end{array}$

H $\quad 2.75711 \quad 4.91713 \quad 1.35047$
\end{abstract}

\section{Cis TS1 (B3LYP 6-31G*(THF))}

$\mathrm{E}\left(\mathrm{B} 3 \mathrm{LYP} 6-31 \mathrm{G}^{*}(\mathrm{THF})\right)=$
-1302.67564

$\begin{array}{cccc}\mathrm{O} & -.37405 & 1.36227 & -.19669 \\ \mathrm{C} & -.19730 & .63292 & .82916 \\ \mathrm{C} & 1.84876 & .37888 & 1.18703 \\ \mathrm{P} & 2.47403 & -.20865 & -.32721 \\ \mathrm{C} & -.64552 & 1.12107 & 2.18253 \\ \mathrm{C} & -.83285 & .22855 & 3.24589 \\ \mathrm{C} & -1.30068 & .68299 & 4.48022 \\ \mathrm{C} & -1.58647 & 2.04056 & 4.66252 \\ \mathrm{C} & -1.40120 & 2.93655 & 3.60502 \\ \mathrm{C} & -.93524 & 2.47753 & 2.37283 \\ \mathrm{O} & 2.83022 & .88405 & -1.42429 \\ \mathrm{C} & 1.93960 & 1.65970 & -2.28055 \\ \mathrm{O} & 3.87265 & -.95086 & -.10532 \\ \mathrm{C} & 4.57079 & -1.63358 & -1.18634 \\ \mathrm{O} & 1.57027 & -1.36987 & -.94691 \\ \mathrm{C} & .45269 & -1.20897 & -1.87142 \\ \mathrm{H} & -. .32237 & -.46458 & .74097 \\ \mathrm{H} & 1.84040 & -.47275 & 1.87355 \\ \mathrm{H} & -.62225 & -.83049 & 3.10016 \\ \mathrm{H} & -1.45187 & -.01950 & 5.29688 \\ \mathrm{H} & -1.95693 & 2.39608 & 5.62153 \\ \mathrm{H} & -1.62824 & 3.99201 & 3.73958 \\ \mathrm{H} & -.80363 & 3.15543 & 1.53601 \\ \mathrm{H} & 1.76720 & 1.09912 & -3.20193 \\ \mathrm{H} & 2.48120 & 2.57983 & -2.50432 \\ \mathrm{H} & 1.00165 & 1.86576 & -1.76341 \\ \mathrm{H} & 3.96316 & -2.46237 & -1.55630 \\ \mathrm{H} & 4.79441 & -.92986 & -1.99139 \\ \mathrm{H} & 5.49751 & -2.00949 & -.75199 \\ \mathrm{H} & -.14370 & -2.11713 & -1.77386 \\ \mathrm{H} & .84639 & -1.13699 & -2.88853 \\ \mathrm{H} & -.13527 & -.32630 & -1.60796 \\ \mathrm{C} & 3.41888 & 3.93715 & 3.05532 \\ \mathrm{C} & 3.37747 & 2.72129 & 3.74423 \\ \mathrm{C} & 2.89829 & 1.57175 & 3.11754 \\ \mathrm{C} & 2.43976 & 1.60126 & 1.78535 \\ \mathrm{C} & 2.47025 & 2.83857 & 1.11101 \\ \mathrm{C} & 2.95998 & 3.98568 & 1.73660 \\ \mathrm{H} & 3.79855 & 4.83343 & 3.54022\end{array}$




\begin{abstract}
$\begin{array}{llll}\text { H } & 3.72325 & 2.66507 & 4.77446\end{array}$
H $2.87327 \quad 63158 \quad 3.66429$

$\begin{array}{llll}\mathrm{H} & 2.08285 & 2.91164 & .10120\end{array}$

H $2.97294 \quad 4.92664 \quad 1.19027$
\end{abstract}

\section{Trans TS1 (B3LYP 6-31G*)}

$\mathrm{E}\left(\mathrm{B} 3 \mathrm{LYP} 6-31 \mathrm{G}^{*}\right)=-1302.66114$ $\mathrm{E}\left(\mathrm{B} 3 \mathrm{LYP} 6-31 \mathrm{G}^{*}(\mathrm{THF})\right)=$ $-1302.67707$

\begin{tabular}{lccc}
$\mathrm{O}$ & -.25925 & -.00543 & -.22173 \\
$\mathrm{C}$ & -.03593 & -.02890 & 1.04157 \\
$\mathrm{C}$ & 1.92513 & -.10494 & 1.26774 \\
$\mathrm{P}$ & 2.34687 & -1.20063 & -.03943 \\
$\mathrm{C}$ &.- .1706 & 1.17761 & 1.87232 \\
$\mathrm{C}$ & -.49286 & 1.11599 & 3.26980 \\
$\mathrm{C}$ & -.86456 & 2.23950 & 4.00962 \\
$\mathrm{C}$ & -1.17105 & 3.43750 & 3.35998 \\
$\mathrm{C}$ & -1.11286 & 3.50188 & 1.96489 \\
$\mathrm{C}$ & -.74151 & 2.37799 & 1.22772 \\
$\mathrm{O}$ & 3.82038 & -1.76570 & .23218 \\
$\mathrm{C}$ & 4.40314 & -2.80118 & -.58621 \\
$\mathrm{O}$ & 1.51093 & -2.55204 & -.13016 \\
$\mathrm{C}$ & .34081 & -2.78276 & -.96923 \\
$\mathrm{O}$ & 2.37399 & -.58334 & -1.50595 \\
$\mathrm{C}$ & 2.03910 & .78657 & -1.86450 \\
$\mathrm{H}$ & -.22262 & -.97030 & 1.60098 \\
$\mathrm{H}$ & 2.18663 & .91168 & .96882 \\
$\mathrm{H}$ & -.26276 & .18266 & 3.77783 \\
$\mathrm{H}$ & -.91968 & 2.17843 & 5.09370 \\
$\mathrm{H}$ & -1.46373 & 4.31196 & 3.93603 \\
$\mathrm{H}$ & -1.36851 & 4.42668 & 1.45248 \\
$\mathrm{H}$ & -.72313 & 2.39799 & .14227 \\
$\mathrm{H}$ & 4.48458 & -2.46440 & -1.62402 \\
$\mathrm{H}$ & 3.79986 & -3.71038 & -.53086 \\
$\mathrm{H}$ & 5.39662 & -2.98302 & -.17556 \\
$\mathrm{H}$ & .67398 & -2.93612 & -1.99898 \\
$\mathrm{H}$ &.- .32589 & -1.92010 & -.89081 \\
$\mathrm{H}$ & -.11056 & -3.69640 & -.58078 \\
$\mathrm{H}$ & 2.03501 & .80085 & -2.95507 \\
$\mathrm{H}$ & 2.81264 & 1.46330 & -1.48914 \\
$\mathrm{H}$ & 1.05199 & 1.01656 & -1.46015 \\
$\mathrm{C}$ & 3.10649 & -1.04919 & 5.29589 \\
$\mathrm{C}$ & 3.29770 & .23435 & 4.77933 \\
$\mathrm{C}$ & 2.94717 & .52336 & 3.46227 \\
$\mathrm{C}$ & 2.39294 & -.46254 & 2.62649 \\
$\mathrm{C}$ & 2.19450 & -1.74893 & 3.16382 \\
$\mathrm{C}$ & 2.55321 & -2.03775 & 4.47873 \\
$\mathrm{H}$ & 3.38138 & -1.27542 & 6.32250 \\
$\mathrm{H}$ & 3.72289 & 1.01615 & 5.40382 \\
& 3.09544 & 1.52703 & 3.07118 \\
$\mathrm{H}$ & 1.75050 & -2.52732 & 2.54811 \\
\hline & -3.03994 & 4.86856
\end{tabular}

Trans TS1 (B3LYP 6-31G*(THF))

$\mathrm{E}(\mathrm{B} 3 \mathrm{LYP} 6-31 \mathrm{G} *(\mathrm{THF}))=$

\begin{abstract}
$-1302.68015$
$\begin{array}{llll}\mathrm{O} & -. .34190 & .04953 & -.22050\end{array}$

$\begin{array}{llll}\text { C } & -.03525 & -.01625 & 1.02492\end{array}$

C $1.93308 \quad-.10753 \quad 1.20010$

$\begin{array}{llll}\text { P } & 2.36852 & -1.23847 & -.07458\end{array}$

$\begin{array}{llll}\text { C } & -.38520 & 1.16617 & 1.90775\end{array}$

C $\quad \begin{array}{llll}-.57470 & 1.02278 & 3.28833\end{array}$

C $\quad \begin{array}{llll}.95396 & 2.11557 & 4.07213\end{array}$

C $-1.15107 \quad 3.36729 \quad 3.48250$

C $\quad \begin{array}{llll}\text { C.97024 } & 3.51785 & 2.10312\end{array}$

C $\quad \begin{array}{llll}-.59393 & 2.42312 & 1.32436\end{array}$

$\begin{array}{llll}\text { O } & 3.86380 & -1.75873 & .15443\end{array}$

C $4.44913-2.79413 \quad-.68608$

$\begin{array}{llll}\text { O } & 1.54440 & -2.59773 & -.09388\end{array}$

$\begin{array}{llll}\text { C } & .33965 & -2.85877 & -.88208\end{array}$

$\begin{array}{lllll}\text { O } & 2.27278 & -.65207 & -1.54704\end{array}$

C $2.13455 \quad 75900 \quad-1.88963$

$\begin{array}{llll}\mathrm{H} & -.20925 & -.97087 & 1.56654\end{array}$

$\begin{array}{llll}\mathrm{H} & 2.21205 & .89881 & .88014\end{array}$

$\begin{array}{llll}\mathrm{H} & -.43627 & .04691 & 3.74808\end{array}$

$\mathrm{H}-1.10433 \quad 1.98806 \quad 5.14176$

H $-1.45411 \quad 4.21696 \quad 4.09037$

$\mathrm{H}-1.13618 \quad 4.48623 \quad 1.63513$

$\begin{array}{llll}\mathrm{H} & -.48337 & 2.51782 & .24777\end{array}$

H $4.48944 \quad-2.45479-1.72438$

H $3.86530-3.71320 \quad-.60573$

H $5.45827-2.95292 \quad-.30560$

$\mathrm{H} \quad .64126 \quad-3.17680 \quad-1.88279$

H $\quad-.27255 \quad-1.95579 \quad-.91208$

H $\quad-.17316 \quad-3.67196 \quad-.36654$

H $2.11549 \quad .79311 \quad-2.97943$

H $2.99434 \quad 1.32194 \quad-1.51701$

H $1.19451 \quad 1.11633 \quad-1.46961$

$\begin{array}{llll}\text { C } & 3.03099 & -.91946 & 5.28710\end{array}$

$\begin{array}{llll}\text { C } & 3.23576 & .34564 & 4.72909\end{array}$

$\begin{array}{llll}\text { C } & 2.91523 & .58739 & 3.39400\end{array}$

$\begin{array}{llll}\text { C } & 2.37759 & -.42694 & 2.58009\end{array}$

C $2.16931 \quad-1.69540 \quad 3.15744$

$\begin{array}{llll}\text { C } & 2.49732 & -1.93787 & 4.49175\end{array}$

H $3.28253 \quad-1.10887 \quad 6.32767$

H $3.64812 \quad 1.14945 \quad 5.33480$

$\begin{array}{llll}\text { H } & 3.07504 & 1.57681 & 2.97167\end{array}$

H $1.74140 \quad-2.49724 \quad 2.56063$

H $2.32972-2.927314 .91178$
\end{abstract}

Cis OP1 (B3LYP 6-31G*)

E(B3LYP 6-31G*) $=-1302.70768$

$\mathrm{E}\left(\mathrm{B} 3 \mathrm{LYP} \quad 6-31 \mathrm{G}^{*}(\mathrm{THF})\right)=$ 1302.71922

$\begin{array}{llll}\text { O } & .38880 & .44004 & -.35137\end{array}$

$\begin{array}{llll}\text { C } & .30191 & .42986 & 1.07563\end{array}$

$\begin{array}{llll}\text { C } & 1.83643 & .54394 & 1.35187\end{array}$

$\begin{array}{llll}\mathrm{P} & 2.06978 & .86650 & -.48161\end{array}$

$\begin{array}{llll}\text { C } & -.62191 & 1.49304 & 1.63322\end{array}$

$\begin{array}{llll}\text { C } & -.97147 & 1.46183 & 2.99006\end{array}$

$\begin{array}{cccc}\mathrm{C} & -1.83021 & 2.42028 & 3.52538 \\ \mathrm{C} & -2.35963 & 3.42069 & 2.70600 \\ \mathrm{C} & -2.02633 & 3.44895 & 1.35125 \\ \mathrm{C} & -1.16416 & 2.48919 & .81520 \\ \mathrm{O} & 1.69642 & 2.38354 & -.97252 \\ \mathrm{C} & 2.65786 & 3.40253 & -1.27013 \\ \mathrm{O} & 2.30053 & -.12295 & -1.77144 \\ \mathrm{C} & 1.23625 & -.59639 & -2.60774 \\ \mathrm{O} & 3.72944 & 1.01644 & -.28322 \\ \mathrm{C} & 4.72106 & .87031 & -1.29366 \\ \mathrm{H} & -.06916 & -.55061 & 1.40645 \\ \mathrm{H} & 2.22882 & -.46717 & 1.51500 \\ \mathrm{H} & -.56389 & .68410 & 3.63304 \\ \mathrm{H} & -2.08735 & 2.38616 & 4.58100 \\ \mathrm{H} & -3.03114 & 4.16791 & 3.12112 \\ \mathrm{H} & -2.44190 & 4.21851 & .70520 \\ \mathrm{H} & -.90243 & 2.50184 & -.23687 \\ \mathrm{H} & 3.45754 & 3.42771 & -.52740 \\ \mathrm{H} & 2.10859 & 4.34701 & -1.25712 \\ \mathrm{H} & 3.08337 & 3.25207 & -2.26823 \\ \mathrm{H} & .59282 & -1.29180 & -2.06624 \\ \mathrm{H} & .62916 & .23304 & -2.98150 \\ \mathrm{H} & 1.72364 & -1.10336 & -3.44488 \\ \mathrm{H} & 5.66768 & 1.19438 & -.84916 \\ \mathrm{H} & 4.51730 & 1.48458 & -2.17898 \\ \mathrm{H} & 4.80601 & -.17296 & -1.61186 \\ \mathrm{C} & 3.63808 & 3.11278 & 4.32504 \\ \mathrm{C} & 3.88825 & 1.74029 & 4.32210 \\ \mathrm{C} & 3.29449 & .92510 & 3.35820 \\ \mathrm{C} & 2.44341 & 1.45876 & 2.38025 \\ \mathrm{C} & 2.20120 & 2.84273 & 2.39370 \\ \mathrm{C} & 2.79246 & 3.65899 & 3.35684 \\ \mathrm{H} & 4.09721 & 3.75185 & 5.07477 \\ \mathrm{H} & 4.54455 & 1.30182 & 5.06948 \\ \mathrm{H} & 3.49728 & -.14371 & 3.35978 \\ \mathrm{H} & 1.54278 & 3.28096 & 1.65077 \\ \mathrm{H} & 2.58936 & 4.72684 & 3.35177\end{array}$

Trans OP1 (B3L YP 6-31G*)

$\mathrm{E}\left(\mathrm{B} 3 \mathrm{LYP} 6-31 \mathrm{G}^{*}\right)=-1302.71365$ E(B3LYP $6-31 G^{*}($ THF $\left.)\right)=$ $-1302.72559$

$\begin{array}{lccc}\mathrm{O} & .44450 & .65078 & -.76894 \\ \mathrm{C} & .53908 & .20076 & .58474 \\ \mathrm{C} & 2.02272 & .58955 & .81165 \\ \mathrm{P} & 2.19042 & .76283 & -1.04356 \\ \mathrm{C} & -.46571 & .84846 & 1.50978 \\ \mathrm{C} & -.62515 & .34666 & 2.80909 \\ \mathrm{C} & -1.50993 & .95312 & 3.69948 \\ \mathrm{C} & -2.25485 & 2.06582 & 3.29922 \\ \mathrm{C} & -2.10895 & 2.56181 & 2.00328 \\ \mathrm{C} & -1.21874 & 1.95698 & 1.11295 \\ \mathrm{O} & 3.82954 & .98096 & -.93978 \\ \mathrm{C} & 4.67613 & 1.12518 & -2.07540 \\ \mathrm{O} & 2.31879 & -.62304 & -1.90566 \\ \mathrm{C} & 1.25611 & -1.21235 & -2.66329\end{array}$




$\begin{array}{cccc}\mathrm{O} & 2.03135 & 2.07064 & -2.02061 \\ \mathrm{C} & .79881 & 2.58553 & -2.53431 \\ \mathrm{H} & .42490 & -.89263 & .63907 \\ \mathrm{H} & 2.06417 & 1.60748 & 1.21736 \\ \mathrm{H} & -.05097 & -.52269 & 3.12277 \\ \mathrm{H} & -1.62146 & .55514 & 4.70481 \\ \mathrm{H} & -2.94700 & 2.53752 & 3.99178 \\ \mathrm{H} & -2.69206 & 3.42072 & 1.68037 \\ \mathrm{H} & -1.11379 & 2.32877 & .09927 \\ \mathrm{H} & 4.51253 & 2.08963 & -2.56786 \\ \mathrm{H} & 4.51252 & .31633 & -2.79606 \\ \mathrm{H} & 5.70571 & 1.07414 & -1.70917 \\ \mathrm{H} & 1.12593 & -.67710 & -3.61085 \\ \mathrm{H} & .31591 & -1.20030 & -2.10889 \\ \mathrm{H} & 1.56380 & -2.23939 & -2.87399 \\ \mathrm{H} & 1.08136 & 3.32237 & -3.29110 \\ \mathrm{H} & .22114 & 3.07306 & -1.74528 \\ \mathrm{H} & .18650 & 1.80508 & -2.99222 \\ \mathrm{C} & 4.59890 & -1.97718 & 3.14002 \\ \mathrm{C} & 4.45476 & -.63008 & 3.47328 \\ \mathrm{C} & 3.62741 & .19473 & 2.70990 \\ \mathrm{C} & 2.93432 & -.30749 & 1.60072 \\ \mathrm{C} & 3.08929 & -1.66419 & 1.27462 \\ \mathrm{C} & 3.91294 & -2.49054 & 2.03652 \\ \mathrm{H} & 5.24178 & -2.62252 & 3.73282 \\ \mathrm{H} & 4.98454 & -.21894 & 4.32867 \\ \mathrm{H} & 3.51689 & 1.24349 & 2.97590 \\ \mathrm{H} & 2.57746 & -2.06989 & .40646 \\ \mathrm{H} & 4.02300 & -3.53753 & 1.76576\end{array}$

\section{Cis OP2 (B3LYP 6-31G*)}

E(B3LYP 6-31G*) $=-1302.70541$ $\mathrm{E}(\mathrm{B} 3 \mathrm{LYP} 6-31 \mathrm{G} *(\mathrm{THF}))=$ $-1302.71617$

$\begin{array}{llll}\mathrm{O} & .22436 & .74265 & -.32543 \\ \mathrm{C} & .14083 & .59051 & 1.11246 \\ \mathrm{C} & 1.67862 & .57375 & 1.38589 \\ \mathrm{P} & 1.89058 & .94290 & -.48228 \\ \mathrm{C} & -.72665 & 1.65185 & 1.74975 \\ \mathrm{C} & -1.06400 & 1.53317 & 3.10523 \\ \mathrm{C} & -1.86546 & 2.48987 & 3.72470 \\ \mathrm{C} & -2.34845 & 3.57902 & 2.99446 \\ \mathrm{C} & -2.02756 & 3.69682 & 1.64199 \\ \mathrm{C} & -1.22340 & 2.73735 & 1.02122 \\ \mathrm{O} & 2.66524 & 2.35805 & -.25665 \\ \mathrm{C} & 2.96303 & 3.35968 & -1.23839 \\ \mathrm{O} & 2.89352 & -.35591 & -.66655 \\ \mathrm{C} & 3.61459 & -.67300 & -1.86358 \\ \mathrm{O} & 1.70608 & 1.24236 & -2.12989 \\ \mathrm{C} & .74797 & .56126 & -2.93041 \\ \mathrm{H} & -.29809 & -.39207 & 1.32458 \\ \mathrm{H} & 1.97903 & -.46962 & 1.51043 \\ \mathrm{H} & -.68850 & .68974 & 3.68055 \\ \mathrm{H} & -2.11247 & 2.38645 & 4.77809 \\ \mathrm{H} & -2.97406 & 4.32572 & 3.47652 \\ \mathrm{H} & -2.40758 & 4.53518 & 1.06348\end{array}$

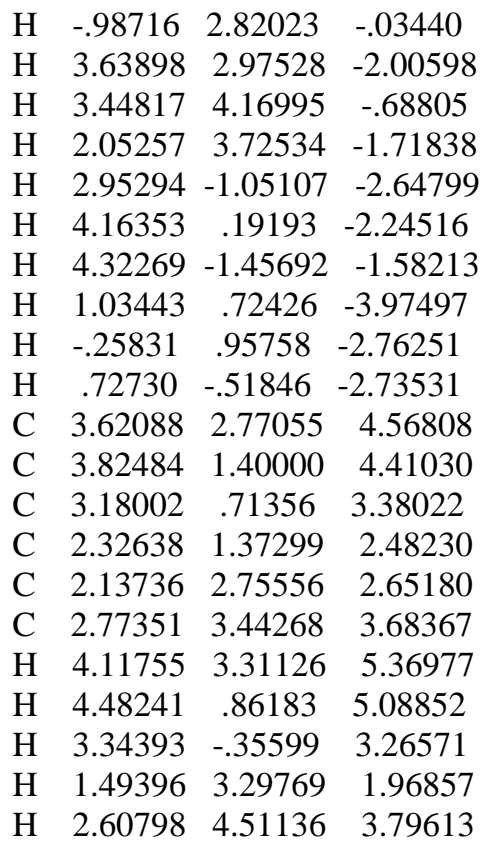

\section{Trans OP2 (B3LYP 6-31G*)}

$\mathrm{E}(\mathrm{B} 3 \mathrm{LYP}$ 6-31G*) $=-1302.70990$ $\mathrm{E}(\mathrm{B} 3 \mathrm{LYP}$ 6-31G*(THF) $)=$ $-1302.72039$

$\begin{array}{cccc}\mathrm{O} & .37129 & .86040 & -.64335 \\ \mathrm{C} & .46261 & .26154 & .67538 \\ \mathrm{C} & 1.97642 & .49640 & .87804 \\ \mathrm{P} & 2.02410 & .98999 & -.96943 \\ \mathrm{C} & -. .6291 & .91383 & 1.67283 \\ \mathrm{C} & -.84742 & .20206 & 2.81616 \\ \mathrm{C} & -1.65226 & .79863 & 3.78636 \\ \mathrm{C} & -2.09171 & 2.11378 & 3.61936 \\ \mathrm{C} & -1.71963 & 2.82490 & 2.47701 \\ \mathrm{C} & -.90860 & 2.23006 & 1.50927 \\ \mathrm{O} & 3.03657 & 2.24424 & -.65552 \\ \mathrm{C} & 3.46724 & 3.26024 & -1.57202 \\ \mathrm{O} & 2.78093 & -.38095 & -1.45169 \\ \mathrm{C} & 3.25521 & -.65075 & -2.77532 \\ \mathrm{O} & 1.69060 & 1.59578 & -2.50017 \\ \mathrm{C} & .55453 & 1.20710 & -3.26446 \\ \mathrm{H} & .22409 & -.80512 & .59588 \\ \mathrm{H} & 2.11225 & 1.42600 & 1.43778 \\ \mathrm{H} & -.50996 & -.82396 & 2.94742 \\ \mathrm{H} & -1.94092 & .23456 & 4.66950 \\ \mathrm{H} & -2.72374 & 2.57840 & 4.37160 \\ \mathrm{H} & -2.06484 & 3.84603 & 2.33542 \\ \mathrm{H} & -.62947 & 2.77883 & .61521 \\ \mathrm{H} & 2.62019 & 3.83866 & -1.94600 \\ \mathrm{H} & 4.00909 & 2.83246 & -2.41998 \\ \mathrm{H} & 4.13754 & 3.90623 & -.99863 \\ \mathrm{H} & 3.83201 & .18657 & -3.17557 \\ \mathrm{H} & 2.43106 & -.87721 & -3.45820 \\ \mathrm{H} & 3.89999 & -1.52848 & -2.68531 \\ \mathrm{H} & .74370 & 1.53076 & -4.29324\end{array}$

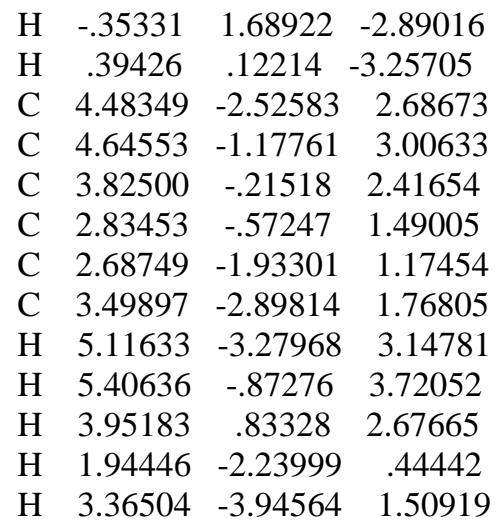

\section{Cis TS2 (B3LYP 6-31G*)}

$\mathrm{E}\left(\mathrm{B} 3 \mathrm{LYP} 6-31 \mathrm{G}^{*}\right)=-1302.65887$ $\mathrm{E}\left(\right.$ B3LYP $\left.6-31 \mathrm{G}^{*}(\mathrm{THF})\right)=$ $-1302.67043$

$\begin{array}{lccc}\text { C } & -.09560 & -.00723 & -.01034 \\ \text { C } & -.19390 & -.03129 & 1.41777 \\ \text { P } & 2.23723 & -.09037 & 1.13717 \\ \text { O } & 1.67802 & .06843 & -.31091 \\ \text { C } & -.61305 & 1.09469 & -.89169 \\ \text { C } & -1.95868 & 1.46263 & -.74493 \\ \text { C } & -2.52771 & 2.41684 & -1.58733 \\ \text { C } & -1.75866 & 3.02776 & -2.57965 \\ \text { C } & -.41890 & 2.66851 & -2.72943 \\ \text { C } & .14945 & 1.70321 & -1.89752 \\ \text { O } & 2.22656 & .98554 & 2.32813 \\ \text { C } & 3.21288 & 2.02057 & 2.53203 \\ \text { O } & 2.16974 & -1.59871 & 1.72067 \\ \text { C } & 3.02469 & -1.97907 & 2.81535 \\ \text { O } & 3.85271 & .04307 & .84546 \\ \text { C } & 4.45755 & -.58552 & -.28715 \\ \text { H } & -.19021 & -.98830 & -.47453 \\ \text { H } & -. .39197 & -1.01836 & 1.82760 \\ \text { H } & -2.55303 & 1.00452 & .03977 \\ \text { H } & -3.57093 & 2.69171 & -1.45820 \\ \text { H } & -2.19928 & 3.77954 & -3.22909 \\ \text { H } & .19106 & 3.13927 & -3.49655 \\ \text { H } & 1.19064 & 1.42746 & -2.01119 \\ \text { H } & 4.12925 & 1.59783 & 2.95021 \\ \text { H } & 2.74922 & 2.71153 & 3.23792 \\ \text { H } & 3.44422 & 2.53766 & 1.59882 \\ \text { H } & 4.06642 & -1.72051 & 2.60601 \\ \text { H } & 2.69731 & -1.48600 & 3.73577 \\ \text { H } & 2.92065 & -3.06051 & 2.91655 \\ \text { H } & 5.53099 & -.39358 & -.20852 \\ \text { H } & 4.06914 & -.16392 & -1.21769 \\ \text { H } & 4.28333 & -1.66789 & -.28035 \\ \text { C } & -1.33701 & 3.10508 & 4.14158 \\ \text { C } & -1.55759 & 1.75947 & 4.44247 \\ \text { C } & -1.20448 & .76698 & 3.53094 \\ \text { C } & -.61180 & 1.07548 & 2.28978 \\ \text { C } & -.38925 & 2.44095 & 2.00822 \\ \text { C } & -.75119 & 3.43385 & 2.91608 \\ & & & \end{array}$




\begin{abstract}
H $-1.61196 \quad 3.88268 \quad 4.84931$
$\begin{array}{llll}\mathrm{H} & -2.00935 & 1.47911 & 5.39148\end{array}$

H $-1.38236 \quad-.27771 \quad 3.77985$

$\mathrm{H} \quad .08560 \quad 2.72801 \quad 1.07505$

$\begin{array}{llll}\mathrm{H} & -.56642 & 4.47620 & 2.66487\end{array}$
\end{abstract}

\section{Trans TS2 (B3LYP 6-31G*)}

$\mathrm{E}\left(\right.$ B3LYP $\left.6-31 \mathrm{G}^{*}\right)=-1302.66482$ $\mathrm{E}(\mathrm{B} 3 \mathrm{LYP} 6-31 \mathrm{G} *(\mathrm{THF}))=$ $-1302.67676$

$\begin{array}{llll}\text { C } & -.34414 & .01759 & .07734 \\ \text { C } & -.43802 & -.00094 & 1.50874 \\ \text { P } & 1.97631 & -.05663 & 1.24295 \\ \text { O } & 1.42345 & -.22781 & -.20483 \\ \text { C } & -.54788 & 1.23906 & -.74423 \\ \text { C } & -.94511 & 1.09437 & -2.08048 \\ \text { C } & -1.09454 & 2.20790 & -2.90655 \\ \text { C } & -.84017 & 3.48539 & -2.40647 \\ \text { C } & -.43995 & 3.64038 & -1.07615 \\ \text { C } & -.29819 & 2.52918 & -.24981 \\ \text { O } & 1.87602 & 1.27655 & 2.16130 \\ \text { C } & 2.99832 & 2.14881 & 2.45456 \\ \text { O } & 2.02291 & -1.42333 & 2.10165 \\ \text { C } & 2.21892 & -1.41182 & 3.52299 \\ \text { O } & 3.58430 & .11580 & .93706 \\ \text { C } & 4.26595 & -.83246 & .11250 \\ \text { H } & -. .63700 & -.89102 & -.44458 \\ \text { H } & -.68919 & .94288 & 1.98333 \\ \text { H } & -1.11896 & .09701 & -2.47779 \\ \text { H } & -1.39649 & 2.07562 & -3.94203 \\ \text { H } & -.94213 & 4.35467 & -3.05052 \\ \text { H } & -.23095 & 4.63224 & -.68394 \\ \text { H } & -.03798 & 2.65671 & .77399 \\ \text { H } & 3.42343 & 2.55963 & 1.53791 \\ \text { H } & 3.77559 & 1.61542 & 3.00577 \\ \text { H } & 2.57853 & 2.94485 & 3.07262 \\ \text { H } & 1.39504 & -.89257 & 4.01797 \\ \text { H } & 3.17772 & -.94708 & 3.77996 \\ \text { H } & 2.22219 & -2.45858 & 3.83038 \\ \text { H } & 5.31929 & -.54018 & .10928 \\ \text { H } & 3.87098 & -.81161 & -.90713 \\ \text { H } & 4.17326 & -1.84528 & .52120 \\ \text { C } & -1.75459 & -3.44957 & 3.73694 \\ \text { C } & -1.90740 & -2.15855 & 4.24671 \\ \text { C } & -1.49800 & -1.05181 & 3.50418 \\ \text { C } & -.91274 & -1.19201 & 2.22882 \\ \text { C } & -.76540 & -2.50575 & 1.73339 \\ \text { C } & -1.18235 & -3.61030 & 2.47214 \\ \text { H } & -2.07058 & -4.31432 & 4.31428 \\ \text { H } & -2.35374 & -2.00997 & 5.22743 \\ \text { H } & -1.63209 & -.05145 & 3.91199 \\ \text { H } & -.27961 & -2.66934 & .77504 \\ \text { H } & -1.04545 & -4.60819 & 2.06096 \\ & & & \end{array}$

PO(OMe $)_{3}($ B3LYP 6-31G*)

\begin{tabular}{|c|c|c|}
\hline P 0.06116 & -0.32566 & -0.38870 \\
\hline O 1.26134 & 0.74905 & -0.2122 \\
\hline C 2.05991 & 1.08 & -1 . \\
\hline O -1.25484 & & \\
\hline C -1.6 & & \\
\hline $\mathrm{O}-0.2 \mathrm{C}$ & & \\
\hline C 0.75965 & -1.5 & 1. \\
\hline Н 1.47403 & 1.66 & -2.08351 \\
\hline H 2.43665 & 0.17681 & -1.84370 \\
\hline H 2.88903 & 1.68988 & -0.99413 \\
\hline H $\quad-0.88195$ & 2.30569 & 0.3106 \\
\hline H -2.04823 & 1.19215 & 1.08681 \\
\hline H -2.51212 & 2.08748 & -0.39132 \\
\hline H $\quad 0.31105$ & -1.83 & \\
\hline H 1.68493 & -0.99 & 1.9942 \\
\hline H 0.96998 & -2.45618 & 1.2380 \\
\hline
\end{tabular}

\section{G. $\mathrm{Me}_{3} \mathrm{PCHCOOMe} \mathrm{+}$ PhCHO (reaction 6)}

\section{$\mathrm{Me}_{3}$ PCHCOOMe (B3LYP 6- 31G*)}

E(B3LYP 6-31G*) $=-728.2609$
E(B3LYP 6-31G*(THF)) $=$
-728.276701

$\begin{array}{cccc}\mathrm{P} & .34568 & -.29784 & -1.58171 \\ \mathrm{C} & .48347 & -.20005 & .11960 \\ \mathrm{H} & .26860 & .72131 & .64478 \\ \mathrm{C} & .89755 & -1.39071 & .77357 \\ \mathrm{O} & .99859 & -1.26319 & 2.13581 \\ \mathrm{O} & 1.15483 & -2.46671 & .20953 \\ \mathrm{C} & 1.41194 & -2.44439 & 2.82175 \\ \mathrm{H} & 1.43694 & -2.17704 & 3.88054 \\ \mathrm{H} & .70861 & -3.26697 & 2.65718 \\ \mathrm{H} & 2.40389 & -2.76965 & 2.49190 \\ \mathrm{C} & 1.89508 & -.72318 & -2.47269 \\ \mathrm{H} & 1.72455 & -.86007 & -3.54595 \\ \mathrm{H} & 2.63337 & .06807 & -2.31357 \\ \mathrm{H} & 2.27073 & -1.65191 & -2.03521 \\ \mathrm{C} & -.86665 & -1.53123 & -2.20144 \\ \mathrm{H} & -.84394 & -1.61423 & -3.29330 \\ \mathrm{H} & -.60292 & -2.49162 & -1.75059 \\ \mathrm{H} & -1.87100 & -1.24875 & -1.87273 \\ \mathrm{C} & -.19201 & 1.32476 & -2.23201 \\ \mathrm{H} & -.28420 & 1.29210 & -3.32157 \\ \mathrm{H} & -1.16011 & 1.59226 & -1.79928 \\ \mathrm{H} & .53895 & 2.09077 & -1.95814\end{array}$

$\mathrm{Me}_{3}$ PCHCOOMe (B3LYP 631G*(THF))

\begin{abstract}
E(B3LYP
$\left.6-31 G^{*}(\mathrm{THF})\right)=$ 728.27725

$\begin{array}{llll}\mathrm{P} & .34285 & -.29850 & -1.61357\end{array}$

$\begin{array}{llll}\text { C } & .49609 & -.24005 & .10494\end{array}$

$\begin{array}{llll}\mathrm{H} & .28168 & .68333 & .63029\end{array}$

$\begin{array}{llll}\text { C } & .90572 & -1.41102 & .77805\end{array}$

$\begin{array}{llll}\text { O } & .99847 & -1.25372 & 2.14601\end{array}$

$\begin{array}{llll}\mathrm{O} & 1.17243 & -2.51016 & .25324\end{array}$

$\begin{array}{llll}\text { C } & 1.40575 & -2.41482 & 2.87874\end{array}$

H $1.42130 \quad-2.111093 .92802$

H $\quad \begin{array}{llll}70125 & -3.24124 & 2.74181\end{array}$

$\begin{array}{llll}\mathrm{H} & 2.40210 & -2.75045 & 2.57395\end{array}$

$\begin{array}{llll}\text { C } & 1.89181 & -.70020 & -2.50814\end{array}$

$\begin{array}{lllll}\mathrm{H} & 1.70733 & -.79684 & -3.58334\end{array}$

$\begin{array}{llll}\mathrm{H} & 2.63017 & .08836 & -2.33190\end{array}$

$\begin{array}{llll}\mathrm{H} & 2.27847 & -1.64528 & -2.11626\end{array}$

$\begin{array}{llll}\text { C } & -.88070 & -1.51574 & -2.23269\end{array}$

$\begin{array}{lllll}\mathrm{H} & -.85568 & -1.57632 & -3.32574\end{array}$

$\begin{array}{lllll}\mathrm{H} & -.63554 & -2.49231 & -1.80664\end{array}$

H $-1.88311-1.22469-1.90358$

C $-.19710 \quad 1.34083-2.20571$

$\begin{array}{llll}\mathrm{H} & -.30206 & 1.32938 & -3.29509\end{array}$

$\begin{array}{llll}\mathrm{H} & -1.16088 & 1.59863 & -1.75537\end{array}$

H $\quad .54103 \quad 2.09873-1.92478$
\end{abstract}

\section{Cis TS1 (B3LYP 6-31G*)}

E(B3LYP 6-31G*) $=-1073.81$

$\mathrm{E}(\mathrm{B} 3 \mathrm{LYP} 6-31 \mathrm{G} *(\mathrm{THF}))=$ $-1073.83082$

$\begin{array}{llll}\mathrm{O} & .89328 & 2.11961 & .67201 \\ \mathrm{C} & 1.63943 & 1.54411 & -.21923 \\ \mathrm{P} & -.79025 & .23112 & .29777 \\ \mathrm{C} & .55407 & .20335 & -.92696 \\ \mathrm{C} & 2.94082 & .90092 & .25674 \\ \mathrm{C} & 3.17316 & .74790 & 1.62788 \\ \mathrm{C} & 4.36164 & .18370 & 2.09361 \\ \mathrm{C} & 5.34574 & -.22335 & 1.19078 \\ \mathrm{C} & 5.13747 & -.04607 & -.17992 \\ \mathrm{C} & 3.94573 & .51629 & -.64096 \\ \mathrm{H} & 1.83298 & 2.08836 & -1.16972 \\ \mathrm{H} & .20717 & .62579 & -1.87106 \\ \mathrm{H} & 2.41661 & 1.10763 & 2.31754 \\ \mathrm{H} & 4.52548 & .07020 & 3.16306 \\ \mathrm{H} & 6.27373 & -.66057 & 1.55137 \\ \mathrm{H} & 5.91033 & -.33559 & -.88832 \\ \mathrm{H} & 3.79315 & .66348 & -1.70776 \\ \mathrm{C} & -1.86043 & 1.70140 & .13971 \\ \mathrm{H} & -2.65605 & 1.64880 & .89076 \\ \mathrm{H} & -1.23158 & 2.57842 & .29207 \\ \mathrm{H} & -2.31597 & 1.71528 & -.85572 \\ \mathrm{C} & -1.91606 & -1.16574 & -.10882 \\ \mathrm{H} & -1.36278 & -2.10431 & -.03092 \\ \mathrm{H} & -2.76220 & -1.18175 & .58665 \\ \mathrm{H} & -2.29976 & -1.05865 & -1.12793\end{array}$




\begin{abstract}
$\begin{array}{llll}\text { C } & -.31882 & -.08229 & 2.03256\end{array}$
$\begin{array}{llll}\mathrm{H} & -1.16452 & -.54328 & 2.55438\end{array}$

$\begin{array}{llll}\mathrm{H} & .52348 & -.77780 & 2.02826\end{array}$

$\begin{array}{llll}\mathrm{H} & -.02066 & .85571 & 2.49591\end{array}$

C $1.16335-1.12864-1.06786$

O $1.84818 \quad-1.25029 \quad-2.23112$

$\begin{array}{llll}\mathrm{O} & 1.14361 & -2.01049 & -.21615\end{array}$

C $2.65100 \quad-2.43572 \quad-2.34534$

H $3.11435 \quad-2.37819 \quad-3.33114$

H $3.41433 \quad-2.45145 \quad-1.56295$

H $2.03193 \quad-3.33277 \quad-2.26310$
\end{abstract}

\section{Cis TS1 (B3LYP 6-31G*(THF))}

\section{$\mathrm{E}(\mathrm{B} 3 \mathrm{LYP} 6-31 \mathrm{G} *(\mathrm{THF}))=$} $-1073.83600$

$\begin{array}{cccc}\mathrm{O} & 1.06419 & 1.45826 & 1.54683 \\ \mathrm{C} & 1.52638 & 1.32965 & .34310 \\ \mathrm{P} & -1.08900 & .17326 & .07676 \\ \mathrm{C} & .59291 & .05123 & -.59057 \\ \mathrm{C} & 3.00292 & .96629 & .19820 \\ \mathrm{C} & 3.64705 & .28368 & 1.23697 \\ \mathrm{C} & 5.00588 & -.02567 & 1.15012 \\ \mathrm{C} & 5.74288 & .36158 & .02563 \\ \mathrm{C} & 5.11069 & 1.05814 & -1.00885 \\ \mathrm{C} & 3.74835 & 1.35543 & -.92032 \\ \mathrm{H} & 1.30034 & 2.15495 & -.37480 \\ \mathrm{H} & .59295 & .37691 & -1.63339 \\ \mathrm{H} & 3.06563 & .02262 & 2.11602 \\ \mathrm{H} & 5.49557 & -.55431 & 1.96575 \\ \mathrm{H} & 6.80482 & .13410 & -.03778 \\ \mathrm{H} & 5.68157 & 1.37776 & -1.87817 \\ \mathrm{H} & 3.25960 & 1.90816 & -1.72235 \\ \mathrm{C} & -1.61270 & 1.91700 & .04644 \\ \mathrm{H} & -2.65154 & 1.99174 & .38446 \\ \mathrm{H} & -.95108 & 2.45750 & .72732 \\ \mathrm{H} & -1.53384 & 2.32278 & -.96681 \\ \mathrm{C} & -2.25861 & -.75913 & -.97308 \\ \mathrm{H} & -3.27390 & -.66130 & -.57229 \\ \mathrm{H} & -2.23658 & -.37098 & -1.99632 \\ \mathrm{H} & -1.98253 & -1.81809 & -.98566 \\ \mathrm{C} & -1.25063 & -.47038 & 1.76919 \\ \mathrm{H} & -2.23571 & -.19751 & 2.16287 \\ \mathrm{H} & -1.13227 & -1.55481 & 1.76176 \\ \mathrm{H} & -.45384 & -.01151 & 2.35660 \\ \mathrm{C} & 1.19641 & -1.29006 & -.44421 \\ \mathrm{O} & 2.07707 & -1.54795 & -1.43873 \\ \mathrm{O} & 1.00317 & -2.07022 & .48135 \\ \mathrm{C} & 2.89042 & -2.72444 & -1.26913 \\ \mathrm{H} & 3.52268 & -2.77325 & -2.15635 \\ \mathrm{H} & 3.50445 & -2.63264 & -.36979 \\ \mathrm{H} & 2.26940 & -3.62109 & -1.19604\end{array}$

\section{Trans TS1 (B3LYP 6-31G*)}

$\mathrm{E}(\mathrm{B} 3 \mathrm{LYP}$ 6-31G*) $=-1073.815$ $\mathrm{E}(\mathrm{B} 3 \mathrm{LYP} 6-31 \mathrm{G} *(\mathrm{THF}))=$

$\begin{array}{cccc}-1073.83784 & & \\ \mathrm{C} & .05356 & -.08065 & .16185 \\ \mathrm{O} & -.20076 & -.16938 & 1.43677 \\ \mathrm{P} & 2.44436 & .04335 & 1.51723 \\ \mathrm{C} & 1.74858 & -.59276 & -.03589 \\ \mathrm{C} & -.76384 & -.95114 & -.78738 \\ \mathrm{C} & -.90779 & -.63243 & -2.14186 \\ \mathrm{C} & -1.66922 & -1.44233 & -2.98618 \\ \mathrm{C} & -2.29836 & -2.58197 & -2.47986 \\ \mathrm{C} & -2.17043 & -2.89792 & -1.12430 \\ \mathrm{C} & -1.41159 & -2.08366 & -.28393 \\ \mathrm{C} & 1.95254 & 1.75669 & 1.89185 \\ \mathrm{C} & 2.09888 & -1.09461 & 2.89822 \\ \mathrm{C} & 4.27552 & .08699 & 1.38894 \\ \mathrm{H} & .12546 & .94687 & -.27157 \\ \mathrm{H} & 1.70987 & -1.68244 & -.00716 \\ \mathrm{H} & -.43321 & .26571 & -2.53465 \\ \mathrm{H} & -1.77828 & -1.18133 & -4.03636 \\ \mathrm{H} & -2.89406 & -3.21296 & -3.13505 \\ \mathrm{H} & -2.67261 & -3.77515 & -.72244 \\ \mathrm{H} & -1.32317 & -2.28689 & .77938 \\ \mathrm{H} & 2.53130 & 2.12634 & 2.74443 \\ \mathrm{H} & .88236 & 1.74465 & 2.10429 \\ \mathrm{H} & 2.16703 & 2.36701 & 1.01059 \\ \mathrm{H} & 2.41412 & -.63970 & 3.84301 \\ \mathrm{H} & 2.65415 & -2.02638 & 2.74729 \\ \mathrm{H} & 1.02283 & -1.27505 & 2.88924 \\ \mathrm{H} & 4.70689 & .46593 & 2.32151 \\ \mathrm{H} & 4.56331 & .73940 & .56152 \\ \mathrm{H} & 4.66396 & -.91924 & 1.20447 \\ \mathrm{C} & 2.42013 & -.05220 & -1.23405 \\ \mathrm{O} & 2.36631 & -.90242 & -2.28294 \\ \mathrm{O} & 2.89418 & 1.07687 & -1.31523 \\ \mathrm{C} & 2.84998 & -.38155 & -3.52948 \\ \mathrm{H} & 2.71153 & -1.18550 & -4.25290 \\ \mathrm{H} & 2.27957 & .50277 & -3.82594 \\ \mathrm{H} & 3.90678 & -.11107 & -3.45192 \\ & & & \\ & & & \\ & & \end{array}$

\section{Trans TS1 (B3LYP 6- 31G*(THF))}

\section{$\mathrm{E}(\mathrm{B} 3 \mathrm{LYP} 6-31 \mathrm{G} *(\mathrm{THF}))=$ $-1073.84094$}

$\begin{array}{cccc}\mathrm{C} & .00583 & -.06426 & .17192 \\ \mathrm{O} & -. .36574 & -.12142 & 1.41262 \\ \mathrm{P} & 2.53445 & .06586 & 1.54293 \\ \mathrm{C} & 1.76648 & -.60172 & .04230 \\ \mathrm{C} & -. .74224 & -.95330 & -.81708 \\ \mathrm{C} & -.93863 & -.56848 & -2.14844 \\ \mathrm{C} & -1.68668 & -1.36621 & -3.01922 \\ \mathrm{C} & -2.24821 & -2.56216 & -2.56391 \\ \mathrm{C} & -2.06042 & -2.95151 & -1.23244 \\ \mathrm{C} & -1.31812 & -2.14755 & -.36682 \\ \mathrm{C} & 2.01084 & 1.77351 & 1.88939 \\ \mathrm{C} & 2.10218 & -1.02062 & 2.93607 \\ \mathrm{C} & 4.35657 & .08155 & 1.42043\end{array}$

$$
\begin{array}{cccc}
\mathrm{H} & .13382 & .94941 & -.27483 \\
\mathrm{H} & 1.72257 & -1.68991 & .09650 \\
\mathrm{H} & -.52054 & .37307 & -2.50235 \\
\mathrm{H} & -1.84134 & -1.04928 & -4.04835 \\
\mathrm{H} & -2.83643 & -3.18131 & -3.23763 \\
\mathrm{H} & -2.50549 & -3.87533 & -.86812 \\
\mathrm{H} & -1.19235 & -2.41624 & .67837 \\
\mathrm{H} & 2.50227 & 2.12527 & 2.80228 \\
\mathrm{H} & .92690 & 1.75914 & 2.02105 \\
\mathrm{H} & 2.29029 & 2.41161 & 1.04708 \\
\mathrm{H} & 2.43666 & -.57385 & 3.87810 \\
\mathrm{H} & 2.58627 & -1.99422 & 2.80629 \\
\mathrm{H} & 1.01571 & -1.13160 & 2.92606 \\
\mathrm{H} & 4.78040 & .46503 & 2.35516 \\
\mathrm{H} & 4.66241 & .72502 & .59188 \\
\mathrm{H} & 4.72806 & -.93344 & 1.24936 \\
\mathrm{C} & 2.37910 & -.09008 & -1.19341 \\
\mathrm{O} & 2.29689 & -.96182 & -2.22014 \\
\mathrm{O} & 2.83200 & 1.04625 & -1.32345 \\
\mathrm{C} & 2.70239 & -.46124 & -3.50901 \\
\mathrm{H} & 2.50170 & -1.26955 & -4.21271 \\
\mathrm{H} & 2.12302 & .42648 & -3.77474 \\
\mathrm{H} & 3.76673 & -.21098 & -3.50901
\end{array}
$$

\section{Cis OP1 (B3LYP 6-31G*)}

$\mathrm{E}\left(\mathrm{B} 3 \mathrm{LYP} 6-31 \mathrm{G}^{*}\right)=-1073.83099$ $\mathrm{E}($ B3LYP 6-31G* $(\mathrm{THF}))=$ $-1073.84386$

$$
\begin{array}{lccc}
\mathrm{O} & .40660 & .81006 & -.85263 \\
\mathrm{C} & .40855 & .53997 & .52449 \\
\mathrm{C} & 1.93632 & .76882 & .80159 \\
\mathrm{P} & 2.24956 & 1.06726 & -1.03729 \\
\mathrm{C} & -.55917 & 1.39467 & 1.32954 \\
\mathrm{C} & -.81834 & 1.09017 & 2.67320 \\
\mathrm{C} & -1.69845 & 1.87094 & 3.42231 \\
\mathrm{C} & -2.33989 & 2.96344 & 2.83232 \\
\mathrm{C} & -2.09565 & 3.26352 & 1.49146 \\
\mathrm{C} & -1.21005 & 2.48378 & .74459 \\
\mathrm{H} & .17376 & -.51841 & .73425 \\
\mathrm{H} & -.32775 & .23561 & 3.13532 \\
\mathrm{H} & -1.89062 & 1.62220 & 4.46321 \\
\mathrm{H} & -3.03047 & 3.56993 & 3.41282 \\
\mathrm{H} & -2.59849 & 4.10602 & 1.02297 \\
\mathrm{H} & -1.02306 & 2.69763 & -.30212 \\
\mathrm{H} & 2.45026 & -.13253 & 1.15110 \\
\mathrm{C} & 2.33230 & -.40106 & -2.14959 \\
\mathrm{H} & 3.24483 & -.40484 & -2.75208 \\
\mathrm{H} & 2.31576 & -1.30723 & -1.53428 \\
\mathrm{H} & 1.44442 & -.41507 & -2.78401 \\
\mathrm{C} & 1.92712 & 2.65070 & -1.91758 \\
\mathrm{H} & 2.77639 & 2.93604 & -2.54495 \\
\mathrm{H} & 1.01505 & 2.55589 & -2.50914 \\
\mathrm{H} & 1.77744 & 3.41869 & -1.15345 \\
\mathrm{C} & 4.12920 & 1.30624 & -.87006 \\
\mathrm{H} & 4.60155 & 1.44075 & -1.85037 \\
\mathrm{H} & 4.35133 & 2.18958 & -.26171
\end{array}
$$




\begin{abstract}
H $\quad 4.59106 \quad .43394 \quad-.38994$
$\begin{array}{llll}\text { C } & 2.28105 & 1.92186 & 1.71104\end{array}$

$\begin{array}{llll}\text { O } & 2.46913 & 1.50028 & 2.97937\end{array}$

$\begin{array}{llll}\text { O } & 2.34929 & 3.08875 & 1.37918\end{array}$

C $2.67359 \quad 2.54255 \quad 3.95042$

$\begin{array}{llll}\mathrm{H} & 2.82758 & 2.02913 & 4.89984\end{array}$

H $3.548063 .14366 \quad 3.68937$

H $1.79333 \quad 3.18832 \quad 3.99916$
\end{abstract}

\section{Trans OP1 (B3LYP 6-31G*)}

E(B3LYP 6-31G*) $=-1073.83456$
E(B3LYP 6-31G*(THF) $)=$
-1073.84782

$\begin{array}{llll}\mathrm{O} & .52109 & 1.14044 & -.84158 \\ \mathrm{C} & .35121 & .61366 & .46265 \\ \mathrm{C} & 1.81513 & .73244 & .93608 \\ \mathrm{P} & 2.34983 & 1.05382 & -.87786 \\ \mathrm{C} & -.65908 & 1.37385 & 1.30045 \\ \mathrm{C} & -1.15416 & .80719 & 2.48240 \\ \mathrm{C} & -2.05551 & 1.50762 & 3.28358 \\ \mathrm{C} & -2.48151 & 2.78382 & 2.90649 \\ \mathrm{C} & -2.00198 & 3.34835 & 1.72347 \\ \mathrm{C} & -1.09541 & 2.64779 & .92496 \\ \mathrm{H} & .06548 & -.44927 & .43780 \\ \mathrm{H} & 1.99823 & 1.63832 & 1.52168 \\ \mathrm{H} & -.83534 & -.19243 & 2.77307 \\ \mathrm{H} & -2.43114 & 1.05548 & 4.19814 \\ \mathrm{H} & -3.18824 & 3.32919 & 3.52672 \\ \mathrm{H} & -2.33896 & 4.33576 & 1.41715 \\ \mathrm{H} & -.73185 & 3.06891 & -.00665 \\ \mathrm{C} & 2.43664 & -.46055 & 1.59954 \\ \mathrm{O} & 3.32172 & -.10314 & 2.55707 \\ \mathrm{O} & 2.20841 & -1.61854 & 1.30345 \\ \mathrm{C} & 4.00179 & -1.19130 & 3.20543 \\ \mathrm{H} & 3.28451 & -1.85018 & 3.70146 \\ \mathrm{H} & 4.57434 & -1.77429 & 2.47894 \\ \mathrm{H} & 4.66593 & -.72708 & 3.93480 \\ \mathrm{C} & 2.34464 & -.40279 & -1.99898 \\ \mathrm{H} & 3.29575 & -.51128 & -2.52753 \\ \mathrm{H} & 2.18071 & -1.29343 & -1.38263 \\ \mathrm{H} & 1.51510 & -.31266 & -2.70247 \\ \mathrm{C} & 2.36045 & 2.67580 & -1.76691 \\ \mathrm{H} & 3.28351 & 2.81551 & -2.33613 \\ \mathrm{H} & 1.48561 & 2.73481 & -2.41670 \\ \mathrm{H} & 2.28450 & 3.47820 & -1.02481 \\ \mathrm{C} & 4.21971 & 1.05263 & -.51366 \\ \mathrm{H} & 4.80173 & 1.20791 & -1.42980 \\ \mathrm{H} & 4.48002 & 1.84757 & .19582 \\ \mathrm{H} & 4.53093 & .09819 & -.07378\end{array}$

\section{Cis OP2 (B3LYP 6-31G*)}

$\mathrm{E}\left(\mathrm{B} 3 \mathrm{LYP} 6-31 \mathrm{G}^{*}\right)=-1073.82955$

$\mathrm{E}(\mathrm{B} 3 \mathrm{LYP} 6-31 \mathrm{G} *(\mathrm{THF}))=$ $-1073.84218$

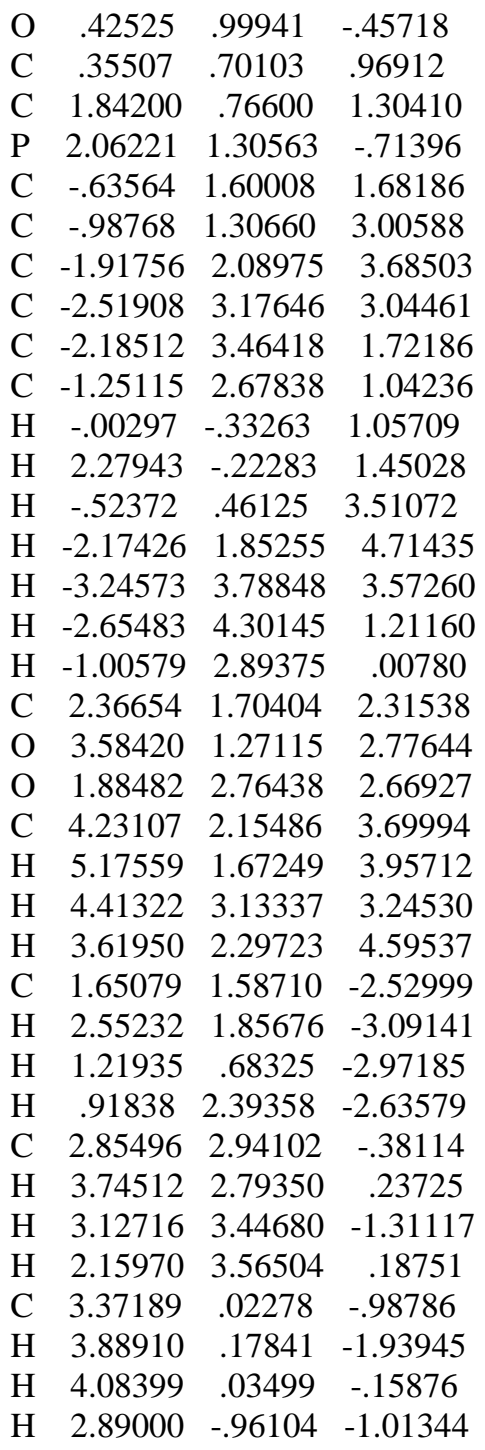

\section{Trans OP2 (B3LYP 6-31G*)}

E(B3LYP 6-31G*) $=-1073.83702$

$\mathrm{E}\left(\mathrm{B} 3 \mathrm{LYP} 6-31 \mathrm{G}^{*}(\mathrm{THF})\right)=$ $-1073.84955$

$\begin{array}{lccl}\mathrm{O} & .41102 & 1.08990 & -.52044 \\ \mathrm{C} & .22135 & .62762 & .85479 \\ \mathrm{C} & 1.66089 & .57663 & 1.31613 \\ \mathrm{P} & 2.07762 & 1.24259 & -.64895 \\ \mathrm{C} & -.70369 & 1.55696 & 1.61521 \\ \mathrm{C} & -.82495 & 1.42677 & 3.00596 \\ \mathrm{C} & -1.68648 & 2.25391 & 3.72528 \\ \mathrm{C} & -2.43966 & 3.22741 & 3.06413 \\ \mathrm{C} & -2.32721 & 3.36024 & 1.68011 \\ \mathrm{C} & -1.46726 & 2.52832 & .95878 \\ \mathrm{H} & -.20838 & -.37885 & .80286 \\ \mathrm{H} & -.23931 & .67333 & 3.52797 \\ \mathrm{H} & -1.76718 & 2.14129 & 4.80345 \\ \mathrm{H} & -3.10877 & 3.87524 & 3.62448 \\ \mathrm{H} & -2.91145 & 4.11271 & 1.15616\end{array}$

$\begin{array}{cccc}\mathrm{H} & -1.38197 & 2.62830 & -.11809 \\ \mathrm{H} & 1.94003 & 1.33894 & 2.04451 \\ \mathrm{C} & 1.82455 & 1.88529 & -2.39900 \\ \mathrm{H} & 2.78820 & 2.10425 & -2.87247 \\ \mathrm{H} & 1.29587 & 1.14323 & -3.00554 \\ \mathrm{H} & 1.22365 & 2.80036 & -2.38899 \\ \mathrm{C} & 3.05900 & 2.68132 & -.01842 \\ \mathrm{H} & 3.74978 & 2.34556 & .75920 \\ \mathrm{H} & 3.60501 & 3.17888 & -.82577 \\ \mathrm{H} & 2.36378 & 3.40076 & .42786 \\ \mathrm{C} & 3.20370 & -.16150 & -1.06168 \\ \mathrm{H} & 3.97269 & -.25041 & -.28936 \\ \mathrm{H} & 2.62120 & -1.08792 & -1.06232 \\ \mathrm{H} & 3.66791 & -.02468 & -2.04198 \\ \mathrm{C} & 2.17822 & -.74997 & 1.69580 \\ \mathrm{O} & 1.70453 & -1.83102 & 1.38354 \\ \mathrm{O} & 3.34087 & -.64630 & 2.40703 \\ \mathrm{C} & 3.94604 & -1.89427 & 2.76877 \\ \mathrm{H} & 4.84465 & -1.63364 & 3.33019 \\ \mathrm{H} & 3.26851 & -2.48934 & 3.38743 \\ \mathrm{H} & 4.20620 & -2.47554 & 1.87916\end{array}$

\section{Cis TS2 (B3LYP 6-31G*)}

$\mathrm{E}\left(\mathrm{B} 3 \mathrm{LYP} 6-31 \mathrm{G}^{*}\right)=-1073.82126$ $\mathrm{E}\left(\right.$ B3LYP $\left.6-31 \mathrm{G}^{*}(\mathrm{THF})\right)=$ $-1073.83923$

$\begin{array}{cccc}\mathrm{O} & .50038 & 1.01644 & -.66777 \\ \mathrm{C} & .38601 & .69609 & .86814 \\ \mathrm{C} & 1.74269 & .55437 & 1.40834 \\ \mathrm{P} & 1.96244 & 1.52744 & -1.07773 \\ \mathrm{C} & -.59521 & 1.68842 & 1.46698 \\ \mathrm{C} & -.75352 & 1.72040 & 2.85960 \\ \mathrm{C} & -1.70865 & 2.54534 & 3.44817 \\ \mathrm{C} & -2.52218 & 3.35970 & 2.65625 \\ \mathrm{C} & -2.37592 & 3.33029 & 1.27054 \\ \mathrm{C} & -1.42511 & 2.49393 & .67932 \\ \mathrm{H} & -.10289 & -.28018 & .79160 \\ \mathrm{H} & 2.15018 & -.45204 & 1.39843 \\ \mathrm{H} & -.11374 & 1.10252 & 3.48173 \\ \mathrm{H} & -1.80988 & 2.56158 & 4.53030 \\ \mathrm{H} & -3.26183 & 4.00961 & 3.11694 \\ \mathrm{H} & -3.00576 & 3.95496 & .64161 \\ \mathrm{H} & -1.33051 & 2.46501 & -.40061 \\ \mathrm{C} & 2.41649 & 1.47610 & 2.25256 \\ \mathrm{O} & 3.59301 & .92332 & 2.74820 \\ \mathrm{O} & 2.13256 & 2.65295 & 2.51129 \\ \mathrm{C} & 4.36949 & 1.79969 & 3.56016 \\ \mathrm{H} & 5.24379 & 1.22134 & 3.86869 \\ \mathrm{H} & 4.68556 & 2.69046 & 3.00585 \\ \mathrm{H} & 3.80906 & 2.13073 & 4.44041 \\ \mathrm{C} & 1.64119 & 1.76817 & -2.86961 \\ \mathrm{H} & 2.51840 & 2.20539 & -3.35841 \\ \mathrm{H} & 1.41276 & .80913 & -3.34402 \\ \mathrm{H} & .78586 & 2.43461 & -3.00835 \\ \mathrm{C} & 2.41956 & 3.18334 & -.48433 \\ \mathrm{H} & 3.45391 & 3.39435 & -.77832\end{array}$




$\begin{array}{cccc}\mathrm{H} & 1.75659 & 3.90956 & -.96550 \\ \mathrm{H} & 2.31628 & 3.26378 & .60625 \\ \mathrm{C} & 3.39960 & .39054 & -1.09058 \\ \mathrm{H} & 4.01833 & .59140 & -1.97505 \\ \mathrm{H} & 3.98132 & .49900 & -.17509 \\ \mathrm{H} & 3.02825 & -.63655 & -1.14559\end{array}$

\section{Trans TS2 (B3LYP 6-31G*)}

$\mathrm{E}\left(\mathrm{B} 3 \mathrm{LYP} 6-31 \mathrm{G}^{*}\right)=-1073.82847$

$\mathrm{E}(\mathrm{B} 3 \mathrm{LYP} 6-31 \mathrm{G} *(\mathrm{THF}))=$ $-1073.84757$

$\begin{array}{cccc}\mathrm{O} & .60204 & 1.43873 & -.53124 \\ \mathrm{C} & .38122 & .75191 & .88637 \\ \mathrm{C} & 1.66848 & .60523 & 1.55104 \\ \mathrm{P} & 2.12633 & 1.40099 & -.99662 \\ \mathrm{C} & -.68189 & 1.60517 & 1.54882 \\ \mathrm{C} & -.75142 & 1.64969 & 2.94718 \\ \mathrm{C} & -1.75479 & 2.37755 & 3.58938 \\ \mathrm{C} & -2.70435 & 3.07519 & 2.84248 \\ \mathrm{C} & -2.64590 & 3.03352 & 1.44781 \\ \mathrm{C} & -1.64666 & 2.30162 & .80643 \\ \mathrm{H} & -.01665 & -.22061 & .58474 \\ \mathrm{H} & -.01514 & 1.10435 & 3.52965 \\ \mathrm{H} & -1.79063 & 2.39949 & 4.67564 \\ \mathrm{H} & -3.48400 & 3.64514 & 3.34137 \\ \mathrm{H} & -3.38166 & 3.57198 & .85546 \\ \mathrm{H} & -1.60503 & 2.27775 & -.27741 \\ \mathrm{H} & 1.99659 & 1.38654 & 2.22940 \\ \mathrm{C} & 1.93686 & 2.28133 & -2.59369 \\ \mathrm{H} & 2.88624 & 2.28896 & -3.13977 \\ \mathrm{H} & 1.17662 & 1.78513 & -3.20269 \\ \mathrm{H} & 1.62054 & 3.31376 & -2.41755 \\ \mathrm{C} & 3.38991 & 2.40814 & -.13280 \\ \mathrm{H} & 3.95328 & 1.79052 & .56659 \\ \mathrm{H} & 4.05713 & 2.87247 & -.87080 \\ \mathrm{H} & 2.88245 & 3.19483 & .43178 \\ \mathrm{C} & 2.77098 & -.23854 & -1.45072 \\ \mathrm{H} & 3.86094 & -.18449 & -1.54542 \\ \mathrm{H} & 2.50683 & -.99188 & -.69547 \\ \mathrm{H} & 2.34174 & -.52367 & -2.41670 \\ \mathrm{C} & 2.24516 & -.68215 & 1.70637 \\ \mathrm{O} & 1.92425 & -1.72899 & 1.12223 \\ \mathrm{O} & 3.32685 & -.66931 & 2.57026 \\ \mathrm{C} & 3.98087 & -1.92510 & 2.73350 \\ \mathrm{H} & 4.79201 & -1.74712 & 3.44368 \\ \mathrm{H} & 3.29700 & -2.68383 & 3.12705 \\ \mathrm{H} & 4.38785 & -2.29396 & 1.78533\end{array}$

Cis methyl-cinnamate (B3LYP 631G*)

$\mathrm{E}\left(\right.$ B3LYP $\left.6-31 \mathrm{G}^{*}\right)=-537.5243$

$\mathrm{E}(\mathrm{B} 3 \mathrm{LYP} 6-31 \mathrm{G} *(\mathrm{THF}))=$ $-537.53310$

$\begin{array}{llll}\text { C } & -.07548 & -2.68804 & .06788\end{array}$

$\begin{array}{lccc}\mathrm{C} & -.44619 & -4.05038 & .08029 \\ \mathrm{C} & -1.78136 & -4.43918 & .04493 \\ \mathrm{C} & -2.78488 & -3.46931 & -.00485 \\ \mathrm{C} & -2.43665 & -2.11650 & -.01899 \\ \mathrm{C} & -1.10143 & -1.72185 & .01678 \\ \mathrm{H} & .33128 & -4.81009 & .11891 \\ \mathrm{H} & -2.03856 & -5.49478 & .05611 \\ \mathrm{H} & -3.83015 & -3.76576 & -.03289 \\ \mathrm{H} & -3.21351 & -1.35747 & -.05820 \\ \mathrm{H} & -.83785 & -.67248 & .00559 \\ \mathrm{C} & 2.14674 & -1.30634 & .12992 \\ \mathrm{C} & 1.36340 & -2.41223 & .11040 \\ \mathrm{H} & 1.94326 & -3.33536 & .13492 \\ \mathrm{H} & 3.21855 & -1.47551 & .16836 \\ \mathrm{C} & 1.80115 & .12610 & .11475 \\ \mathrm{O} & 2.94566 & .85887 & .17216 \\ \mathrm{O} & .70362 & .65476 & .06043 \\ \mathrm{C} & 2.76213 & 2.28019 & .16451 \\ \mathrm{H} & 3.76524 & 2.70509 & .21448 \\ \mathrm{H} & 2.25470 & 2.59989 & -.74995 \\ \mathrm{H} & 2.16713 & 2.59812 & 1.02523\end{array}$

Trans methyl-cinnamate (B3L YP 6-31G*)

\section{$\mathrm{E}\left(\mathrm{B} 3 \mathrm{LYP} 6-31 \mathrm{G}^{*}\right)=-537.5313$ \\ $\mathrm{E}(\mathrm{B} 3 \mathrm{LYP} 6-31 \mathrm{G} *(\mathrm{THF}))=$ $-537.541940$}

$$
\begin{array}{cccc}
\mathrm{C} & .14711 & -.50528 & -.00174 \\
\mathrm{C} & -.51055 & .73787 & .00142 \\
\mathrm{C} & -1.90207 & .81476 & .00677 \\
\mathrm{C} & -2.66445 & -.35383 & .00889 \\
\mathrm{C} & -2.02565 & -1.59858 & .00421 \\
\mathrm{C} & -.63733 & -1.67498 & -.00104 \\
\mathrm{H} & .08284 & 1.64906 & -.00007 \\
\mathrm{H} & -2.38994 & 1.78555 & .00930 \\
\mathrm{H} & -3.74959 & -.29839 & .01343 \\
\mathrm{H} & -2.61508 & -2.51139 & .00468 \\
\mathrm{H} & -.15646 & -2.64875 & -.00443 \\
\mathrm{C} & 2.42003 & -1.59760 & -.00600 \\
\mathrm{C} & 1.61008 & -.52284 & -.00478 \\
\mathrm{H} & 2.09890 & .45035 & -.00549 \\
\mathrm{H} & 2.05237 & -2.61852 & -.00607 \\
\mathrm{C} & 3.88523 & -1.42016 & -.00642 \\
\mathrm{O} & 4.51390 & -2.62342 & -.00505 \\
\mathrm{O} & 4.48012 & -.35843 & -.00720 \\
\mathrm{C} & 5.94651 & -2.56220 & -.00229 \\
\mathrm{H} & 6.28471 & -3.59906 & -.00155 \\
\mathrm{H} & 6.31231 & -2.03960 & -.89045
\end{array}
$$$$
\begin{array}{lllll}
\mathrm{H} & 6.30874 & -2.03958 & .88732
\end{array}
$$

\section{H. $\mathbf{P h}_{3} \mathbf{P C H C O O M e ~ + ~}$ PhCHO (reaction 7)}

$\mathrm{Ph}_{3} \mathrm{PCHCOOMe}$ (B3LYP 631G*)

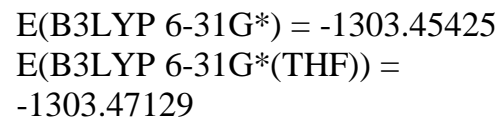

$\mathrm{Ph}_{3} \mathrm{PCHCOOMe}$ (B3LYP 631G*(THF)) $\mathrm{E}(\mathrm{B} 3 \mathrm{LYP} 6-31 \mathrm{G} *(\mathrm{THF}))=$
-1303.47310

$\begin{array}{llll}\text { C } & .39038 & .13271 & .42312\end{array}$

$\begin{array}{llll}\mathrm{P} & .42723 & -.15498 & 2.12374\end{array}$

$\begin{array}{llll}\mathrm{H} & -. .32438 & .86024 & .05938\end{array}$ 


$\begin{array}{cccc}\text { C } & 4.34330 & 1.05640 & 4.30836 \\ \text { C } & 3.10878 & 1.40904 & 4.85751 \\ \text { C } & 1.92406 & 1.04537 & 4.21121 \\ \text { C } & 1.96915 & .32203 & 3.00861 \\ \text { C } & 3.21425 & -.03475 & 2.46085 \\ \text { C } & 4.39247 & .33497 & 3.11113 \\ \text { H } & 5.26429 & 1.34366 & 4.80984 \\ \text { H } & 3.06390 & 1.97135 & 5.78689 \\ \text { H } & .97089 & 1.33187 & 4.64572 \\ \text { H } & 3.25029 & -.59532 & 1.53094 \\ \text { H } & 5.35201 & .05937 & 2.68071 \\ \text { C } & -.45746 & -4.54535 & 3.30988 \\ \text { C } & .26974 & -3.73129 & 4.18259 \\ \text { C } & .56006 & -2.41154 & 3.83074 \\ \text { C } & .11694 & -1.89770 & 2.60204 \\ \text { C } & -.61025 & -2.71895 & 1.72847 \\ \text { C } & -.89645 & -4.03795 & 2.08372 \\ \text { H } & -.67628 & -5.57479 & 3.58264 \\ \text { H } & .61821 & -4.12433 & 5.13418 \\ \text { H } & 1.13456 & -1.78989 & 4.51166 \\ \text { H } & -.94136 & -2.32518 & .77189 \\ \text { H } & -1.45547 & -4.67068 & 1.39929 \\ \text { C } & -2.95565 & 2.47954 & 3.87009 \\ \text { C } & -2.00656 & 3.02640 & 3.00036 \\ \text { C } & -.98520 & 2.22775 & 2.48533 \\ \text { C } & -.90995 & .86792 & 2.83454 \\ \text { C } & -1.86094 & .32518 & 3.71022 \\ \text { C } & -2.88039 & 1.13102 & 4.22455 \\ \text { H } & -3.75138 & 3.10362 & 4.26909 \\ \text { H } & -2.06016 & 4.07565 & 2.72192 \\ \text { H } & -.25231 & 2.66279 & 1.81130 \\ \text { H } & -1.81539 & -.72301 & 3.98851 \\ \text { H } & -3.61647 & .70107 & 4.89884 \\ \text { C } & 1.21611 & -.58491 & -.47574 \\ \text { O } & .99069 & -.23572 & -1.79284 \\ \text { O } & 2.06344 & -1.44726 & -.19476 \\ \text { C } & 1.79978 & -.91605 & -2.75950 \\ \text { H } & 1.49746 & -.51893 & -3.73110 \\ \text { H } & 2.86471 & -.72424 & -2.59438 \\ \text { H } & 1.63047 & -1.99654 & -2.73179\end{array}$

\section{Cis TS1 (B3LYP 6-31G*)}

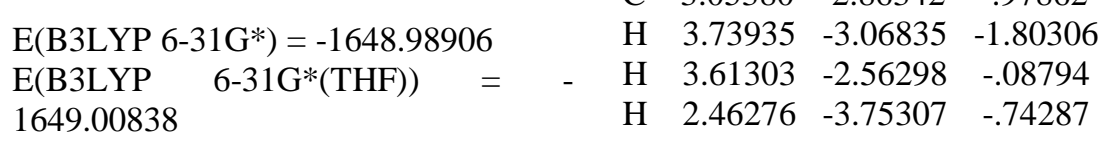

$\begin{array}{lccc}\mathrm{O} & .77740 & 1.46445 & 1.07342 \\ \mathrm{C} & 1.61352 & 1.36442 & .12143 \\ \mathrm{P} & -.93605 & .29841 & -.26032 \\ \mathrm{C} & .66190 & -.13566 & -.97001 \\ \mathrm{C} & 3.02075 & .92493 & .38231 \\ \mathrm{C} & -2.20325 & -.46545 & -1.36762 \\ \mathrm{C} & -1.85622 & -1.43597 & -2.31676 \\ \mathrm{C} & -2.83248 & -1.98519 & -3.15225 \\ \mathrm{C} & -4.15957 & -1.57003 & -3.04760 \\ \mathrm{C} & -4.51378 & -.60229 & -2.10291 \\ \mathrm{C} & -3.54254 & -.05315 & -1.26991\end{array}$

$\begin{array}{cccc}\mathrm{C} & -1.30460 & 2.07646 & -.48378 \\ \mathrm{C} & -1.38992 & -.35275 & 1.39643 \\ \mathrm{C} & -.87527 & 2.69992 & -1.66433 \\ \mathrm{C} & -1.13127 & 4.05637 & -1.87604 \\ \mathrm{C} & -1.83631 & 4.79448 & -.92632 \\ \mathrm{C} & -2.29369 & 4.16764 & .23534 \\ \mathrm{C} & -2.03194 & 2.81687 & .45841 \\ \mathrm{C} & -1.15736 & .34792 & 2.59187 \\ \mathrm{C} & -1.53997 & -.20796 & 3.81201 \\ \mathrm{C} & -2.12829 & -1.47199 & 3.86357 \\ \mathrm{C} & -2.34536 & -2.17896 & 2.68096 \\ \mathrm{C} & -1.98922 & -1.62133 & 1.45381 \\ \mathrm{C} & 3.35118 & .33635 & 1.60991 \\ \mathrm{C} & 4.67000 & -.02906 & 1.87932 \\ \mathrm{C} & 5.66942 & .19125 & .92660 \\ \mathrm{C} & 5.34510 & .77835 & -.30002 \\ \mathrm{C} & 4.02629 & 1.14223 & -.56821 \\ \mathrm{H} & 1.54993 & 2.05862 & -.73937 \\ \mathrm{H} & .71819 & .04708 & -2.04166 \\ \mathrm{H} & -.82503 & -1.76318 & -2.40484 \\ \mathrm{H} & -2.55002 & -2.73556 & -3.88549 \\ \mathrm{H} & -4.91682 & -1.99467 & -3.70085 \\ \mathrm{H} & -5.54538 & -.27172 & -2.01874 \\ \mathrm{H} & -3.82512 & .70671 & -.54603 \\ \mathrm{H} & -. .33771 & 2.13749 & -2.42184 \\ \mathrm{H} & -.77573 & 4.53251 & -2.78580 \\ \mathrm{H} & -2.02904 & 5.85142 & -1.08869 \\ \mathrm{H} & -2.84638 & 4.73475 & .97951 \\ \mathrm{H} & -2.37111 & 2.35062 & 1.37705 \\ \mathrm{H} & -.64370 & 1.29748 & 2.56872 \\ \mathrm{H} & -1.35525 & .34744 & 4.72775 \\ \mathrm{H} & -2.40787 & -1.90614 & 4.81990 \\ \mathrm{H} & -2.79042 & -3.16943 & 2.70805 \\ \mathrm{H} & -2.16636 & -2.18879 & .54745 \\ \mathrm{H} & 2.56067 & .18477 & 2.33738 \\ \mathrm{H} & 4.92280 & -.47948 & 2.83609 \\ \mathrm{H} & 6.69853 & -.08696 & 1.14113 \\ \mathrm{H} & 6.12085 & .95667 & -1.04066 \\ \mathrm{H} & 3.77023 & 1.60386 & -1.52025 \\ \mathrm{C} & 1.27134 & -1.37905 & -.53134 \\ \mathrm{O} & 2.21695 & -1.79348 & -1.43278 \\ \mathrm{O} & 1.06621 & -1.97049 & .52039 \\ \mathrm{C} & 3.05380 & -2.86342 & -.97862 \\ \mathrm{H} & 3.73935 & -3.06835 & -1.80306 \\ \mathrm{H} & 3.61303 & -2.56298 & -.08794 \\ \mathrm{H} & 2.46276 & -3.75307 & -.74287\end{array}$

\section{Cis TS1 (B3LYP 6-31G*(THF))}

$\mathrm{E}\left(\mathrm{B} 3 \mathrm{LYP} 6-31 \mathrm{G}^{*}(\mathrm{THF})\right)=$ $-1649.01803$

$\begin{array}{lccc}\mathrm{O} & .98265 & 1.81749 & 1.00892 \\ \mathrm{C} & 1.66172 & 1.43121 & -.01719 \\ \mathrm{P} & -.97803 & .30045 & -.25647 \\ \mathrm{C} & .73733 & -.02453 & -.78270 \\ \mathrm{C} & 3.09105 & .95369 & .20261 \\ \mathrm{C} & -2.07706 & -.49248 & -1.48930\end{array}$

\begin{abstract}
$\begin{array}{llll}\text { C } & -1.63610 & -1.58490 & -2.25201\end{array}$
C $-2.49474-2.18960-3.17283$

$\begin{array}{llll}\text { C } & -3.79724 & -1.71177 & -3.33689\end{array}$

$\begin{array}{llll}\text { C } & -4.24272 & -.62366 & -2.57962\end{array}$

$\begin{array}{llll}\text { C } & -3.38732 & -.01327 & -1.66268\end{array}$

$\begin{array}{llll}\text { C } & -1.40943 & 2.07034 & -.34248\end{array}$

$\begin{array}{llll}\text { C } & -1.45918 & -.37234 & 1.37735\end{array}$

$\begin{array}{llll}\text { C } & -1.07154 & 2.80725 & -1.48771\end{array}$

$\begin{array}{llll}\text { C } & -1.43755 & 4.15156 & -1.57995\end{array}$

$\begin{array}{llll}\text { C } & -2.15874 & 4.75867 & -.54771\end{array}$

$\begin{array}{llll}\mathrm{C} & -2.52238 & 4.01459 & .57806\end{array}$

$\begin{array}{llll}\text { C } & -2.14931 & 2.67437 & .68352\end{array}$

$\begin{array}{llll}\text { C } & -.80977 & .05506 & 2.54794\end{array}$

$\begin{array}{llll}\text { C } & -1.21628 & -.44274 & 3.78510\end{array}$

$\begin{array}{llll}\text { C } & -2.25813 & -1.37217 & 3.86960\end{array}$

$\begin{array}{llll}\text { C } & -2.89764 & -1.80378 & 2.70635\end{array}$

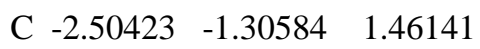

$\begin{array}{llll}\text { C } & 3.48586 & .50595 & 1.46939\end{array}$

$\begin{array}{llll}\text { C } & 4.79681 & .08209 & 1.69802\end{array}$

$\begin{array}{llll}\text { C } & 5.73459 & .11121 & .66081\end{array}$

$\begin{array}{llll}\text { C } & 5.35173 & .56669 & -.60523\end{array}$

$\begin{array}{llll}\text { C } & 4.03804 & .98456 & -.82887\end{array}$

$\begin{array}{llll}\mathrm{H} & 1.61915 & 2.06520 & -.93125\end{array}$

$\begin{array}{llll}\mathrm{H} & .81122 & .05466 & -1.86839\end{array}$

$\begin{array}{llll}\mathrm{H} & -.62464 & -1.96305 & -2.13992\end{array}$

H $-2.14130-3.03206-3.76160$

$\begin{array}{llll}\mathrm{H} & -4.46353 & -2.18214 & -4.05541\end{array}$

$\begin{array}{llll}\mathrm{H} & -5.25381 & -.24549 & -2.70611\end{array}$

$\begin{array}{llll}\mathrm{H} & -3.74044 & .83707 & -1.08578\end{array}$

$\begin{array}{llll}\mathrm{H} & -.52331 & 2.34808 & -2.30620\end{array}$

$\begin{array}{llll}\mathrm{H} & -1.15963 & 4.72372 & -2.46140\end{array}$

$\begin{array}{llll}\mathrm{H} & -2.43908 & 5.80644 & -.62184\end{array}$

$\begin{array}{llll}\mathrm{H} & -3.08782 & 4.47829 & 1.38280\end{array}$

$\begin{array}{llll}\mathrm{H}-2.41582 & 2.11058 & 1.57167\end{array}$

$\begin{array}{llll}\mathrm{H} & .01140 & .75943 & 2.45541\end{array}$

$\begin{array}{llll}\mathrm{H} & -.70955 & -.10863 & 4.68753\end{array}$

$\begin{array}{llll}\text { H } & -2.56682 & -1.76103 & 4.83728\end{array}$

$\begin{array}{llll}\mathrm{H} & -3.70485 & -2.52984 & 2.76136\end{array}$

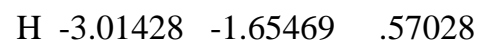

$\begin{array}{llll}\mathrm{H} & 2.75111 & .50540 & 2.26850\end{array}$

$\begin{array}{llll}\mathrm{H} & 5.09059 & -.26141 & 2.68800\end{array}$

$\begin{array}{llll}\mathrm{H} & 6.75822 & -.21131 & .83922\end{array}$

$\begin{array}{llll}\text { H } & 6.07907 & .60269 & -1.41340\end{array}$

$\begin{array}{llll}\mathrm{H} & 3.74222 & 1.34391 & -1.81379\end{array}$

$\begin{array}{llll}\text { C } & 1.29446 & -1.29681 & -.28224\end{array}$

$\begin{array}{llll}\text { O } & 2.15377 & -1.84269 & -1.18413\end{array}$

$\begin{array}{llll}\text { O } & 1.09601 & -1.79024 & .81797\end{array}$

$\begin{array}{llll}\text { C } & 2.93860 & -2.94859 & -.69849\end{array}$

$\begin{array}{llll}\mathrm{H} & 3.55244 & -3.26706 & -1.54202\end{array}$

$\begin{array}{llll}\mathrm{H} & 3.57172 & -2.62628 & .13191\end{array}$

$\begin{array}{llll}\text { H } & 2.29471 & -3.76766 & -.36772\end{array}$
\end{abstract}

\section{Trans TS1 (B3LYP 6-31G*)}

E(B3LYP 6-31G*) $=-1648.995$

$\mathrm{E}($ B3LYP $6-31 \mathrm{G} *($ THF $))=$ $-1649.01594$ 


$\begin{array}{cccc}\text { O } & .88480 & 1.72852 & 1.02580 \\ \text { C } & 1.55597 & .62733 & 1.01573 \\ \text { P } & -.91106 & .31988 & -.13427 \\ \text { C } & .75215 & -.29529 & -.45515 \\ \text { C } & 3.03550 & .67528 & .70078 \\ \text { C } & -2.07603 & -.50692 & -1.29243 \\ \text { C } & -1.60389 & -1.25062 & -2.38167 \\ \text { C } & -2.50023 & -1.77714 & -3.31415 \\ \text { C } & -3.87087 & -1.56640 & -3.16735 \\ \text { C } & -4.34846 & -.82119 & -2.08576 \\ \text { C } & -3.45700 & -.29051 & -1.15678 \\ \text { C } & -1.10397 & 2.07187 & -.60665 \\ \text { C } & -1.52743 & -.05991 & 1.54928 \\ \text { C } & -.47309 & 2.53724 & -1.76863 \\ \text { C } & -.62639 & 3.86495 & -2.16336 \\ \text { C } & -1.41484 & 4.73646 & -1.40953 \\ \text { C } & -2.05773 & 4.26912 & -.26262 \\ \text { C } & -1.90873 & 2.94152 & .13908 \\ \text { C } & -1.22171 & .76036 & 2.64821 \\ \text { C } & -1.67575 & .41450 & 3.92078 \\ \text { C } & -2.41606 & -.75258 & 4.11432 \\ \text { C } & -2.69956 & -1.58226 & 3.02797 \\ \text { C } & -2.26089 & -1.24113 & 1.74913 \\ \text { C } & 3.88389 & -.39173 & 1.01927 \\ \text { C } & 5.24803 & -.32303 & .73305 \\ \text { C } & 5.77777 & .81903 & .12726 \\ \text { C } & 4.93734 & 1.89236 & -.18249 \\ \text { C } & 3.57445 & 1.82120 & .10507 \\ \text { H } & 1.34306 & -.13583 & 1.79463 \\ \text { H } & 1.22478 & .20695 & -1.29551 \\ \text { H } & -.53936 & -1.41729 & -2.50981 \\ \text { H } & -2.12243 & -2.35318 & -4.15429 \\ \text { H } & -4.56655 & -1.97798 & -3.89336 \\ \text { H } & -5.41479 & -.64980 & -1.96753 \\ \text { H } & -3.83740 & .29453 & -.32373 \\ \text { H } & .14999 & 1.87520 & -2.36251 \\ \text { H } & -.12022 & 4.21940 & -3.05717 \\ \text { H } & -1.52467 & 5.77390 & -1.71308 \\ \text { H } & -2.67046 & 4.94139 & .33180 \\ \text { H } & -2.39293 & 2.59784 & 1.04709 \\ \text { H } & -.57767 & 1.61979 & 2.49023 \\ \text { H } & -1.43023 & 1.05346 & 4.76476 \\ \text { H } & -2.75680 & -1.02351 & 5.11022 \\ \text { H } & -3.25486 & -2.50432 & 3.17359 \\ \text { H } & -2.46595 & -1.90871 & .92100 \\ \text { H } & 3.47353 & -1.27635 & 1.50378 \\ \text { H } & 5.89951 & -1.15592 & .98719 \\ \text { H } & 6.84102 & .87563 & -.09372 \\ \text { H } & 5.34958 & 2.78814 & -.64150 \\ \text { H } & 2.90433 & 2.64999 & -.10422 \\ \text { C } & .92745 & -1.74575 & -.42205 \\ \text { O } & 1.99122 & -2.13242 & -1.18215 \\ \text { O } & .28182 & -2.53369 & .25731 \\ \text { C } & 2.36016 & -3.51158 & -1.06436 \\ \text { H } & 3.22688 & -3.63821 & -1.71475 \\ \text { H } & 2.62140 & -3.75704 & -.03100 \\ \text { H } & 1.54206 & -4.16373 & -1.38340 \\ & & & \end{array}$

$\begin{array}{llllll}\text { Trans TS1 (B3LYP } & \text { 6- } & \text { O } & .41982 & -2.40737 & .40568 \\ \text { 31G*(THF)) } & & \text { C } & 2.45011 & -3.38335 & -1.00644 \\ & & \text { H } & 3.27732 & -3.50583 & -1.70661 \\ \text { E(B3LYP 6-31G*(THF)) }= & & \text { H } & 2.78870 & -3.57063 & .01594 \\ -1649.02417 & & \text { H } & 1.64019 & -4.07647 & -1.24918\end{array}$

\section{Cis OP1 (B3LYP 6-31G*)}

$\begin{array}{lcccl}\mathrm{O} & 1.03505 & 1.87032 & 1.03600 & \\ \mathrm{C} & 1.59615 & .70646 & .99963 & \\ \mathrm{P} & -.94873 & .34322 & -.13583 & \mathrm{E}\left(\mathrm{B} 3 \mathrm{LYP} 6-31 \mathrm{G}^{*}\right)=-1649.01646 \\ \mathrm{C} & .76418 & -.18352 & -.40541 & \mathrm{E}\left(\mathrm{B} 3 \mathrm{LYP} 6-31 \mathrm{G}^{*}(\mathrm{THF})\right)= \\ \mathrm{C} & 3.08436 & .63175 & .68126 & -1649.03319\end{array}$

$\begin{array}{cccc}\mathrm{O} & .18679 & .46960 & -.05626 \\ \mathrm{C} & .20506 & .10400 & 1.30485 \\ \mathrm{C} & 1.73034 & .30664 & 1.56273 \\ \mathrm{P} & 2.00204 & .30119 & -.32058 \\ \mathrm{C} & -.77038 & .89319 & 2.15923 \\ \mathrm{C} & -.81571 & .69649 & 3.54646 \\ \mathrm{C} & -1.72287 & 1.40545 & 4.33371 \\ \mathrm{C} & -2.60446 & 2.31349 & 3.74125 \\ \mathrm{C} & -2.57244 & 2.50255 & 2.35905 \\ \mathrm{C} & -1.66033 & 1.79636 & 1.57195 \\ \mathrm{H} & -.01762 & -.97025 & 1.43621 \\ \mathrm{H} & 2.24518 & -.50974 & 2.07615 \\ \mathrm{H} & -.13518 & -.01188 & 4.01461 \\ \mathrm{H} & -1.74405 & 1.24661 & 5.40904 \\ \mathrm{H} & -3.31333 & 2.86499 & 4.35351 \\ \mathrm{H} & -3.25999 & 3.20168 & 1.88955 \\ \mathrm{H} & -1.63170 & 1.92803 & .49579 \\ \mathrm{C} & 1.81774 & 3.56938 & -3.63748 \\ \mathrm{C} & .97105 & 3.65744 & -2.53220 \\ \mathrm{C} & 1.00743 & 2.68121 & -1.53465 \\ \mathrm{C} & 1.91783 & 1.61969 & -1.62185 \\ \mathrm{C} & 2.78793 & 1.55254 & -2.72316 \\ \mathrm{C} & 2.72213 & 2.50934 & -3.73513 \\ \mathrm{H} & 1.77877 & 4.32563 & -4.41732 \\ \mathrm{H} & .27418 & 4.48671 & -2.44371 \\ \mathrm{H} & .34687 & 2.74331 & -.68065 \\ \mathrm{H} & 3.52007 & .75341 & -2.79230 \\ \mathrm{H} & 3.38729 & 2.43362 & -4.59123 \\ \mathrm{C} & 6.74181 & .50090 & .03298 \\ \mathrm{C} & 5.94072 & 1.62399 & .25304 \\ \mathrm{C} & 4.55551 & 1.53578 & .11942 \\ \mathrm{C} & 3.92724 & .32333 & -.22702 \\ \mathrm{C} & 4.74968 & -.79101 & -.45102 \\ \mathrm{C} & 6.14065 & -.70456 & -.32323 \\ \mathrm{H} & 7.82220 & .56863 & .13149 \\ \mathrm{H} & 6.39567 & 2.57490 & .51994 \\ \mathrm{H} & 3.95743 & 2.42952 & .27364 \\ \mathrm{H} & 4.31635 & -1.74204 & -.74160 \\ \mathrm{H} & 6.75114 & -1.58497 & -.50987 \\ \mathrm{C} & 1.23151 & -3.96259 & -1.99748 \\ \mathrm{C} & .99509 & -2.83724 & -2.78980 \\ \mathrm{C} & 1.24774 & -1.55911 & -2.29280 \\ \mathrm{C} & 1.75933 & -1.39043 & -.99832 \\ \mathrm{C} & 1.99280 & -2.52414 & -.20667 \\ \mathrm{C} & 1.72640 & -3.80223 & -.70323 \\ \mathrm{H} & 1.02815 & -4.95704 & -2.38527\end{array}$




\begin{abstract}
H $\quad .60431 \quad-2.95196 \quad-3.79739$
$\mathrm{H} \quad 1.03848 \quad-.69206 \quad-2.91138$

$\begin{array}{llll}\mathrm{H} & 2.39524 & -2.42135 & .79744\end{array}$

H $\quad 1.91050 \quad-4.67052 \quad-.07611$

$\begin{array}{llll}\text { C } & 2.05867 & 1.61834 & 2.23535\end{array}$

$\begin{array}{llll}\text { O } & 2.50130 & 1.41750 & 3.49372\end{array}$

$\begin{array}{llll}\text { O } & 1.91001 & 2.71961 & 1.74591\end{array}$

C $\quad 2.75092 \quad 2.61327 \quad 4.25414$

$\mathrm{H} \quad 3.11211 \quad 2.27083 \quad 5.22410$

H $3.50323 \quad 3.23112 \quad 3.75762$

$\begin{array}{llll}\mathrm{H} & 1.82861 & 3.18880 & 4.36515\end{array}$
\end{abstract}

\section{Trans OP1 (B3LYP 6-31G*)}

$\mathrm{E}($ B3LYP 6-31G*) $=-1649.02074$

$\mathrm{E}(\mathrm{B} 3 \mathrm{LYP} 6-31 \mathrm{G} *(\mathrm{THF}))=$ $-1649.03615$

$\begin{array}{cccc}\text { O } & 1.33478 & .09601 & 3.61098 \\ \mathrm{C} & .50436 & -.91462 & 3.05539 \\ \mathrm{C} & -.42463 & -.01462 & 2.23412 \\ \mathrm{P} & .74227 & 1.48348 & 2.58395 \\ \mathrm{H} & -1.37838 & .19106 & 2.72909 \\ \mathrm{C} & 4.98656 & 2.11101 & .72952 \\ \mathrm{C} & 4.66215 & .91177 & 1.36309 \\ \mathrm{C} & 3.38923 & .71894 & 1.90073 \\ \mathrm{C} & 2.40597 & 1.71484 & 1.78952 \\ \mathrm{C} & 2.74356 & 2.91689 & 1.14274 \\ \mathrm{C} & 4.02447 & 3.11685 & .62889 \\ \mathrm{H} & 5.98166 & 2.26302 & .31957 \\ \mathrm{H} & 5.40375 & .12231 & 1.45119 \\ \mathrm{H} & 3.15573 & -.19355 & 2.43210 \\ \mathrm{H} & 2.00740 & 3.70378 & 1.02753 \\ \mathrm{H} & 4.26513 & 4.05894 & .14344 \\ \mathrm{C} & -2.05836 & 3.99026 & -.32412 \\ \mathrm{C} & -.98961 & 3.25338 & -.83839 \\ \mathrm{C} & -. .12232 & 2.57729 & .02227 \\ \mathrm{C} & -.28606 & 2.62968 & 1.41942 \\ \mathrm{C} & -1.37839 & 3.36106 & 1.91312 \\ \mathrm{C} & -2.25264 & 4.03547 & 1.05594 \\ \mathrm{H} & -2.73293 & 4.51953 & -.99209 \\ \mathrm{H} & -.82456 & 3.20690 & -1.91230 \\ \mathrm{H} & .69491 & 2.00475 & -.40666 \\ \mathrm{H} & -1.55624 & 3.41602 & 2.98314 \\ \mathrm{H} & -3.08422 & 4.59880 & 1.47225 \\ \mathrm{C} & .35532 & 4.15761 & 6.38895 \\ \mathrm{C} & .85350 & 4.69181 & 5.19871 \\ \mathrm{C} & .94749 & 3.89428 & 4.05880 \\ \mathrm{C} & .57804 & 2.54153 & 4.10111 \\ \mathrm{C} & .09601 & 2.00876 & 5.30384 \\ \mathrm{C} & -.03022 & 2.81780 & 6.43502 \\ \mathrm{H} & .27189 & 4.78198 & 7.27471 \\ \mathrm{H} & 1.16402 & 5.73236 & 5.15366 \\ \mathrm{H} & 1.31666 & 4.33257 & 3.13741 \\ \mathrm{H} & -.16447 & .95875 & 5.36306 \\ \mathrm{H} & -.41790 & 2.39202 & 7.35681 \\ \mathrm{C} & -1.29965 & -2.53587 & 3.79833 \\ \mathrm{C} & -.16462 & -1.77589 & 4.11003 \\ & & & \end{array}$

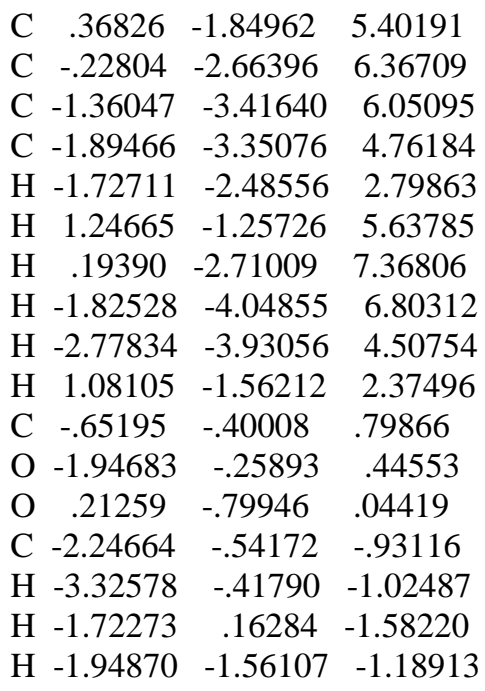

\section{Cis OP2 (B3LYP 6-31G*)}

$\mathrm{E}(\mathrm{B} 3 \mathrm{LYP}$ 6-31G*) $=-1649.01739$

$\mathrm{E}(\mathrm{B} 3 \mathrm{LYP} 6-31 \mathrm{G} *(\mathrm{THF}))=$

$-1649.03207$

$\begin{array}{cccc}\mathrm{O} & .41786 & .340301 & -.06832 \\ \mathrm{C} & .15832 & .259582 & 1.36639 \\ \mathrm{C} & 1.57771 & .327266 & 1.90005 \\ \mathrm{P} & 2.08024 & .193092 & -.18644 \\ \mathrm{C} & -.88948 & 1.263069 & 1.80064 \\ \mathrm{C} & -1.38472 & 1.186649 & 3.10888 \\ \mathrm{C} & -2.37341 & 2.064568 & 3.54735 \\ \mathrm{C} & -2.89009 & 3.027506 & 2.67677 \\ \mathrm{C} & -2.41115 & 3.098025 & 1.36861 \\ \mathrm{C} & -1.41794 & 2.219154 & .93100 \\ \mathrm{H} & -.24375 & -.744921 & 1.55184 \\ \mathrm{H} & 1.87912 & -.558777 & 2.45428 \\ \mathrm{H} & -.98824 & .436528 & 3.79081 \\ \mathrm{H} & -2.74210 & 1.996087 & 4.56774 \\ \mathrm{H} & -3.66231 & 3.713053 & 3.01603 \\ \mathrm{H} & -2.81127 & 3.840081 & .68221 \\ \mathrm{H} & -1.05192 & 2.271221 & -.08889 \\ \mathrm{C} & 1.82696 & .007158 & -4.90293 \\ \mathrm{C} & .65820 & -.111644 & -4.15209 \\ \mathrm{C} & .70555 & -.058376 & -2.75558 \\ \mathrm{C} & 1.92371 & .122383 & -2.08026 \\ \mathrm{C} & 3.09150 & .236690 & -2.85440 \\ \mathrm{C} & 3.04787 & .179068 & -4.24674 \\ \mathrm{H} & 1.78865 & -.030990 & -5.98855 \\ \mathrm{H} & -.29928 & -.244087 & -4.64993 \\ \mathrm{H} & -.21136 & -.149544 & -2.18675 \\ \mathrm{H} & 4.05279 & .383442 & -2.36901 \\ \mathrm{H} & 3.96730 & .274213 & -4.81885 \\ \mathrm{C} & 4.80221 & 3.962882 & -.10035 \\ \mathrm{C} & 5.36759 & 2.714333 & .16317 \\ \mathrm{C} & 4.57615 & 1.563469 & .14122 \\ \mathrm{C} & 3.21040 & 1.653886 & -.15912 \\ \mathrm{C} & 2.64863 & 2.909864 & -.42763 \\ \mathrm{C} & 3.44073 & 4.056353 & -.39453\end{array}$

$\begin{array}{cccc}\mathrm{H} & 5.41899 & 4.857325 & -.07752 \\ \mathrm{H} & 6.42855 & 2.629469 & .38406 \\ \mathrm{H} & 5.03374 & .601215 & .34642 \\ \mathrm{H} & 1.58936 & 2.997702 & -.65001 \\ \mathrm{H} & 2.98950 & 5.024572 & -.59262 \\ \mathrm{C} & 4.28243 & -3.876675 & .41740 \\ \mathrm{C} & 3.39369 & -3.707789 & -.64356 \\ \mathrm{C} & 2.75670 & -2.480382 & -.83931 \\ \mathrm{C} & 2.97784 & -1.413966 & .04504 \\ \mathrm{C} & 3.84796 & -1.605738 & 1.13316 \\ \mathrm{C} & 4.51237 & -2.819431 & 1.30182 \\ \mathrm{H} & 4.78817 & -4.827805 & .56065 \\ \mathrm{H} & 3.19851 & -4.527352 & -1.32988 \\ \mathrm{H} & 2.08727 & -2.357085 & -1.68347 \\ \mathrm{H} & 4.00005 & -.810490 & 1.85648 \\ \mathrm{H} & 5.19756 & -2.944251 & 2.13595 \\ \mathrm{C} & 2.04131 & 1.558767 & 2.56618 \\ \mathrm{O} & 3.13443 & 1.296160 & 3.35003 \\ \mathrm{O} & 1.60562 & 2.684064 & 2.41487 \\ \mathrm{C} & 3.75598 & 2.450184 & 3.92962 \\ \mathrm{H} & 4.57725 & 2.066806 & 4.53785 \\ \mathrm{H} & 4.13658 & 3.115945 & 3.14959 \\ \mathrm{H} & 3.04609 & 3.002219 & 4.55142\end{array}$

\section{Trans OP2 (B3LYP 6-31G*)}

$\mathrm{E}\left(\mathrm{B} 3 \mathrm{LYP} 6-31 \mathrm{G}^{*}\right)=-1649.02599$ E(B3LYP 6-31G*(THF) $)=$ $-1649.04091$

$\begin{array}{cccc}\mathrm{O} & .38555 & .07343 & -.05637 \\ \mathrm{C} & .14516 & .07712 & 1.38420 \\ \mathrm{C} & 1.56854 & -.00207 & 1.89020 \\ \mathrm{P} & 2.05140 & .04073 & -.19163 \\ \mathrm{H} & -.30250 & 1.04193 & 1.64095 \\ \mathrm{H} & 1.84232 & -.96296 & 2.32313 \\ \mathrm{C} & 1.77917 & -.03219 & -4.90877 \\ \mathrm{C} & .62971 & -.27346 & -4.15763 \\ \mathrm{C} & .68045 & -.25399 & -2.76017 \\ \mathrm{C} & 1.88328 & .00821 & -2.08540 \\ \mathrm{C} & 3.03156 & .24856 & -2.85948 \\ \mathrm{C} & 2.98426 & .23003 & -4.25263 \\ \mathrm{H} & 1.73787 & -.04437 & -5.99486 \\ \mathrm{H} & -.31556 & -.47463 & -4.65577 \\ \mathrm{H} & -.22407 & -.43156 & -2.19143 \\ \mathrm{H} & 3.97966 & .46157 & -2.37189 \\ \mathrm{H} & 3.88763 & .42336 & -4.82554 \\ \mathrm{C} & 4.14072 & 4.17910 & .05728 \\ \mathrm{C} & 4.90575 & 3.02741 & .24849 \\ \mathrm{C} & 4.31161 & 1.76518 & .17458 \\ \mathrm{C} & 2.94342 & 1.64924 & -.10250 \\ \mathrm{C} & 2.17917 & 2.80880 & -.29672 \\ \mathrm{C} & 2.77608 & 4.06579 & -.21459 \\ \mathrm{H} & 4.60525 & 5.15949 & .11912 \\ \mathrm{H} & 5.97049 & 3.10626 & .45256 \\ \mathrm{H} & 4.92006 & .87912 & .32538 \\ \mathrm{H} & 1.11677 & 2.72919 & -.51007 \\ \mathrm{H} & 2.17168 & 4.95685 & -.35994\end{array}$




$\begin{array}{cccc}\text { C } & 4.82020 & -3.70022 & .20515 \\ \text { C } & 3.90433 & -3.61270 & -.84238 \\ \text { C } & 3.09658 & -2.48145 & -.97820 \\ \text { C } & 3.17402 & -1.43485 & -.04723 \\ \text { C } & 4.07142 & -1.55055 & 1.02897 \\ \text { C } & 4.90475 & -2.66265 & 1.13717 \\ \text { H } & 5.45808 & -4.57466 & .30228 \\ \text { H } & 3.81948 & -4.42057 & -1.56421 \\ \text { H } & 2.40714 & -2.41877 & -1.81270 \\ \text { H } & 4.11042 & -.78114 & 1.79394 \\ \text { H } & 5.60817 & -2.72647 & 1.96308 \\ \text { C } & -2.44906 & -3.13772 & 2.65696 \\ \text { C } & -2.18552 & -2.05328 & 3.49839 \\ \text { C } & -1.35946 & -1.01733 & 3.06644 \\ \text { C } & -.77875 & -1.05065 & 1.79057 \\ \text { C } & -1.05115 & -2.13526 & .95151 \\ \text { C } & -1.88165 & -3.17267 & 1.38341 \\ \text { H } & -3.09539 & -3.94501 & 2.99135 \\ \text { H } & -2.62741 & -2.01330 & 4.49076 \\ \text { H } & -1.15954 & -.17229 & 3.72228 \\ \text { H } & -.62037 & -2.16243 & -.04405 \\ \text { H } & -2.08641 & -4.00831 & .71875 \\ \text { C } & 2.00950 & 1.12715 & 2.73490 \\ \text { O } & 3.20180 & .85707 & 3.34592 \\ \text { O } & 1.44414 & 2.19881 & 2.86497 \\ \text { C } & 3.75794 & 1.94143 & 4.10188 \\ \text { H } & 4.67567 & 1.55034 & 4.54423 \\ \text { H } & 3.97911 & 2.79046 & 3.44887 \\ \text { H } & 3.06484 & 2.26483 & 4.88292\end{array}$

\section{Cis TS2 (B3L YP 6-31G*)}

$\mathrm{E}\left(\mathrm{B} 3 \mathrm{LYP} 6-31 \mathrm{G}^{*}\right)=-1649.01233$ $\mathrm{E}(\mathrm{B} 3 \mathrm{LYP} 6-31 \mathrm{G} *(\mathrm{THF}))=$ $-1649.03030$

$\begin{array}{lccc}\mathrm{O} & .64609 & .33863 & -.30770 \\ \mathrm{C} & .31713 & .20945 & 1.24451 \\ \mathrm{C} & 1.55550 & .17744 & 2.01073 \\ \mathrm{P} & 2.18155 & .18444 & -.70068 \\ \mathrm{C} & -.74439 & 1.26061 & 1.51794 \\ \mathrm{C} & -1.12851 & 1.47224 & 2.84933 \\ \mathrm{C} & -2.16217 & 2.35129 & 3.16161 \\ \mathrm{C} & -2.82965 & 3.04291 & 2.14744 \\ \mathrm{C} & -2.45568 & 2.83710 & .82064 \\ \mathrm{C} & -1.42625 & 1.94548 & .50624 \\ \mathrm{H} & -.15286 & -.77854 & 1.23937 \\ \mathrm{H} & 1.89003 & -.78828 & 2.36926 \\ \mathrm{H} & -.59962 & .95060 & 3.64171 \\ \mathrm{H} & -2.43971 & 2.50566 & 4.20131 \\ \mathrm{H} & -3.63128 & 3.73540 & 2.39153 \\ \mathrm{H} & -2.96653 & 3.36812 & .02091 \\ \mathrm{H} & -1.14935 & 1.78681 & -.52990 \\ \mathrm{C} & 1.69800 & .18867 & -5.32096 \\ \mathrm{C} & .60095 & -.09914 & -4.50761 \\ \mathrm{C} & .73529 & -.09479 & -3.11793 \\ \mathrm{C} & 1.97417 & .20123 & -2.52881 \\ \mathrm{C} & 3.07213 & .49136 & -3.35352\end{array}$

$\begin{array}{cccc}\mathrm{C} & 2.93372 & .48392 & -4.74115 \\ \mathrm{H} & 1.58984 & .18919 & -6.40225 \\ \mathrm{H} & -.36517 & -.32134 & -4.95254 \\ \mathrm{H} & -.11994 & -.30336 & -2.48525 \\ \mathrm{H} & 4.03398 & .74151 & -2.91567 \\ \mathrm{H} & 3.78987 & .71643 & -5.36841 \\ \mathrm{C} & 4.84301 & 3.87395 & -.02765 \\ \mathrm{C} & 5.44321 & 2.62077 & -.18432 \\ \mathrm{C} & 4.65594 & 1.48307 & -.34936 \\ \mathrm{C} & 3.25384 & 1.59662 & -.35771 \\ \mathrm{C} & 2.65341 & 2.85398 & -.18858 \\ \mathrm{C} & 3.45280 & 3.98542 & -.02880 \\ \mathrm{H} & 5.46024 & 4.75886 & .10228 \\ \mathrm{H} & 6.52585 & 2.52787 & -.17877 \\ \mathrm{H} & 5.13164 & .51473 & -.47061 \\ \mathrm{H} & 1.57379 & 2.94549 & -.15635 \\ \mathrm{H} & 2.98101 & 4.95258 & .11623 \\ \mathrm{C} & 4.29059 & -3.85729 & .09800 \\ \mathrm{C} & 3.41626 & -3.71253 & -.97821 \\ \mathrm{C} & 2.79412 & -2.48516 & -1.21475 \\ \mathrm{C} & 3.02228 & -1.39938 & -.35412 \\ \mathrm{C} & 3.88915 & -1.55677 & .74246 \\ \mathrm{C} & 4.52896 & -2.77563 & .95218 \\ \mathrm{H} & 4.78202 & -4.80991 & .27625 \\ \mathrm{H} & 3.22181 & -4.54920 & -1.64337 \\ \mathrm{H} & 2.13343 & -2.37858 & -2.06915 \\ \mathrm{H} & 4.03796 & -.73954 & 1.44074 \\ \mathrm{H} & 5.20249 & -2.88575 & 1.79736 \\ \mathrm{C} & 2.12848 & 1.32981 & 2.62266 \\ \mathrm{O} & 3.22524 & .98179 & 3.40775 \\ \mathrm{O} & 1.80612 & 2.51031 & 2.48996 \\ \mathrm{C} & 3.90944 & 2.08686 & 3.99219 \\ \mathrm{H} & 4.72190 & 1.65566 & 4.58287 \\ \mathrm{H} & 4.31653 & 2.75552 & 3.22574 \\ \mathrm{H} & 3.24588 & 2.67115 & 4.63779\end{array}$

\section{Trans TS2 (B3LYP 6-31G*)}

$\mathrm{E}(\mathrm{B} 3 \mathrm{LYP}$ 6-31G*) $=-1649.02011$ $\mathrm{E}\left(\mathrm{B} 3 \mathrm{LYP} 6-31 \mathrm{G}^{*}(\mathrm{THF})\right)=$ $-1649.03934$

$\begin{array}{cccc}\mathrm{O} & .66502 & .28728 & -.29557 \\ \mathrm{C} & .48412 & .13364 & 1.28204 \\ \mathrm{C} & 1.80356 & .02716 & 1.89342 \\ \mathrm{P} & 2.17471 & .19035 & -.80579 \\ \mathrm{H} & .00477 & 1.08597 & 1.51182 \\ \mathrm{H} & 2.15040 & -.94257 & 2.22984 \\ \mathrm{C} & 1.29871 & .33030 & -5.37142 \\ \mathrm{C} & .28529 & -.02148 & -4.47859 \\ \mathrm{C} & .53738 & -.05679 & -3.10557 \\ \mathrm{C} & 1.81286 & .26054 & -2.61305 \\ \mathrm{C} & 2.82579 & .61457 & -3.51742 \\ \mathrm{C} & 2.56948 & .64908 & -4.88774 \\ \mathrm{H} & 1.09853 & .36240 & -6.43910 \\ \mathrm{H} & -.70788 & -.26321 & -4.84758 \\ \mathrm{H} & -.25328 & -.31382 & -2.41004 \\ \mathrm{H} & 3.81437 & .88109 & -3.15434\end{array}$

$\begin{array}{cccc}\mathrm{H} & 3.36102 & .93183 & -5.57635 \\ \mathrm{C} & 4.74687 & 3.96526 & -.20791 \\ \mathrm{C} & 5.37965 & 2.74328 & -.45226 \\ \mathrm{C} & 4.62590 & 1.58086 & -.60529 \\ \mathrm{C} & 3.22381 & 1.63646 & -.51270 \\ \mathrm{C} & 2.59234 & 2.86467 & -.26068 \\ \mathrm{C} & 3.35626 & 4.02149 & -.11336 \\ \mathrm{H} & 5.33798 & 4.86901 & -.08718 \\ \mathrm{H} & 6.46268 & 2.69308 & -.52471 \\ \mathrm{H} & 5.12865 & .63755 & -.79413 \\ \mathrm{H} & 1.51412 & 2.91318 & -.16339 \\ \mathrm{H} & 2.85915 & 4.96472 & .09279 \\ \mathrm{C} & 4.54260 & -3.77220 & -.39668 \\ \mathrm{C} & 3.58097 & -3.62113 & -1.39446 \\ \mathrm{C} & 2.87956 & -2.41925 & -1.51577 \\ \mathrm{C} & 3.11693 & -1.36755 & -.61568 \\ \mathrm{C} & 4.07293 & -1.53412 & .40249 \\ \mathrm{C} & 4.79028 & -2.72380 & .49565 \\ \mathrm{H} & 5.09477 & -4.70390 & -.30840 \\ \mathrm{H} & 3.37850 & -4.43201 & -2.08863 \\ \mathrm{H} & 2.14960 & -2.30627 & -2.31069 \\ \mathrm{H} & 4.22946 & -.74788 & 1.13408 \\ \mathrm{H} & 5.53256 & -2.83906 & 1.28041 \\ \mathrm{C} & -2.28865 & -3.13464 & 1.80135 \\ \mathrm{C} & -2.68166 & -1.82211 & 2.06327 \\ \mathrm{C} & -1.78019 & -.77175 & 1.87856 \\ \mathrm{C} & -.47181 & -1.01619 & 1.44483 \\ \mathrm{C} & -.08612 & -2.34057 & 1.18570 \\ \mathrm{C} & -.98714 & -3.38894 & 1.35873 \\ \mathrm{H} & -2.98919 & -3.95423 & 1.93902 \\ \mathrm{H} & -3.69148 & -1.61266 & 2.40674 \\ \mathrm{H} & -2.09210 & .25084 & 2.07779 \\ \mathrm{H} & .92224 & -2.55155 & .84091 \\ \mathrm{H} & -.67281 & -4.40871 & 1.15054 \\ \mathrm{C} & 2.33749 & 1.17986 & 2.54665 \\ \mathrm{O} & 3.50867 & .88916 & 3.22731 \\ \mathrm{O} & 1.89910 & 2.33116 & 2.50853 \\ \mathrm{C} & 4.13451 & 2.01279 & 3.84400 \\ \mathrm{H} & 5.01565 & 1.61743 & 4.35510 \\ \mathrm{H} & 4.43404 & 2.75931 & 3.10062 \\ \mathrm{H} & 3.46602 & 2.49393 & 4.56458 \\ & & & \end{array}$

I.

$(\mathrm{MeO})_{3} \mathrm{PCHCOOMe} \mathrm{+}$ PhCHO (reaction 8)

\section{(MeO) $)_{3}$ PCHCOOMe (B3LYP 6- 31G*)}

$\mathrm{E}\left(\mathrm{B} 3 \mathrm{LYP} 6-31 \mathrm{G}^{*}\right)=-953.94241$ $\mathrm{E}(\mathrm{B} 3 \mathrm{LYP} 6-31 \mathrm{G} *(\mathrm{THF}))=$ $-953.957008$

$\begin{array}{lccc}\mathrm{P} & .37866 & -.06670 & -1.62288 \\ \mathrm{C} & .65814 & -.19598 & .02944 \\ \mathrm{O} & -.11867 & 1.42122 & -1.97484\end{array}$ 


$\begin{array}{cccc}\mathrm{C} & -1.37751 & 1.89643 & -1.45822 \\ \mathrm{O} & -.71386 & -1.03128 & -2.27195 \\ \mathrm{C} & -.42811 & -2.33347 & -2.83972 \\ \mathrm{O} & 1.60416 & -.26834 & -2.65167 \\ \mathrm{C} & 2.94453 & .12026 & -2.30546 \\ \mathrm{H} & .73069 & .70818 & .62097 \\ \mathrm{H} & -1.39485 & 1.83906 & -.36617 \\ \mathrm{H} & -2.19994 & 1.30722 & -1.87376 \\ \mathrm{H} & -1.46471 & 2.93512 & -1.78014 \\ \mathrm{H} & -1.39468 & -2.73000 & -3.15384 \\ \mathrm{H} & .02466 & -2.96701 & -2.07660 \\ \mathrm{H} & .23203 & -2.22312 & -3.70318 \\ \mathrm{H} & 3.61428 & -.54137 & -2.85753 \\ \mathrm{H} & 3.11157 & .01177 & -1.22983 \\ \mathrm{H} & 3.11403 & 1.15795 & -2.60833 \\ \mathrm{C} & .85047 & -1.48686 & .61749 \\ \mathrm{O} & 1.05755 & -1.41571 & 1.96847 \\ \mathrm{O} & .84407 & -2.57003 & .02695 \\ \mathrm{C} & 1.25417 & -2.67522 & 2.61479 \\ \mathrm{H} & 1.40237 & -2.44303 & 3.67130 \\ \mathrm{H} & .38240 & -3.32443 & 2.48857 \\ \mathrm{H} & 2.13148 & -3.19238 & 2.21400\end{array}$

$(\mathrm{MeO})_{3}$ PCHCOOMe (B3LYP 631G*(THF))

$\mathrm{E}(\mathrm{B} 3 \mathrm{LYP} 6-31 \mathrm{G} *(\mathrm{THF}))=-953.95717$

$\begin{array}{lccc}\mathrm{P} & .39093 & -.09254 & -1.63285 \\ \mathrm{C} & .67100 & -.20410 & .02588 \\ \mathrm{O} & -.12069 & 1.38316 & -2.00208 \\ \mathrm{C} & -1.32264 & 1.93304 & -1.40239 \\ \mathrm{O} & -.68692 & -1.10488 & -2.23171 \\ \mathrm{C} & -.38256 & -2.33737 & -2.93983 \\ \mathrm{O} & 1.61033 & -.29400 & -2.66201 \\ \mathrm{C} & 2.93829 & .20615 & -2.37560 \\ \mathrm{H} & .76912 & .71077 & .59899 \\ \mathrm{H} & -1.20931 & 2.01097 & -.31813 \\ \mathrm{H} & -2.18547 & 1.30778 & -1.64768 \\ \mathrm{H} & -1.44729 & 2.92522 & -1.83823 \\ \mathrm{H} & -1.35003 & -2.78627 & -3.17118 \\ \mathrm{H} & .19362 & -2.99419 & -2.28804 \\ \mathrm{H} & .16153 & -2.11827 & -3.86054 \\ \mathrm{H} & 3.62489 & -.37910 & -2.98900 \\ \mathrm{H} & 3.17614 & .07472 & -1.31688 \\ \mathrm{H} & 2.99989 & 1.26309 & -2.64858 \\ \mathrm{C} & .83100 & -1.48174 & .64019 \\ \mathrm{O} & 1.03536 & -1.38869 & 1.99349 \\ \mathrm{O} & .79448 & -2.58282 & .07701 \\ \mathrm{C} & 1.18794 & -2.63602 & 2.68619 \\ \mathrm{H} & 1.37311 & -2.37247 & 3.72947 \\ \mathrm{H} & .28086 & -3.24331 & 2.60891 \\ \mathrm{H} & 2.03123 & -3.20838 & 2.28869\end{array}$

Cis TS1 (B3LYP 6-31G*)

$\mathrm{E}\left(\right.$ B3LYP $\left.6-31 \mathrm{G}^{*}\right)=-1299.48815$

$\mathrm{E}(\mathrm{B} 3 \mathrm{LYP} 6-31 \mathrm{G} *(\mathrm{THF}))=$
$-1299.50585$

$\begin{array}{cccc}\mathrm{O} & -.28973 & .85973 & -.15820 \\ \mathrm{C} & -.11171 & .33181 & 1.00212 \\ \mathrm{C} & 1.78492 & .18043 & 1.25771 \\ \mathrm{P} & 2.28325 & -.24741 & -.38777 \\ \mathrm{C} & -.61046 & 1.07854 & 2.22263 \\ \mathrm{C} & -. .77663 & .43834 & 3.45563 \\ \mathrm{C} & -1.23446 & 1.14634 & 4.56669 \\ \mathrm{C} & -1.53211 & 2.50720 & 4.45285 \\ \mathrm{C} & -1.37693 & 3.15008 & 3.22172 \\ \mathrm{C} & -.92118 & 2.43802 & 2.11160 \\ \mathrm{O} & 2.51458 & .86222 & -1.48036 \\ \mathrm{C} & 1.78388 & 2.08436 & -1.81348 \\ \mathrm{O} & 3.73200 & -.90550 & -.21867 \\ \mathrm{C} & 4.46697 & -1.41662 & -1.34988 \\ \mathrm{O} & 1.44493 & -1.46019 & -.98797 \\ \mathrm{C} & .34888 & -1.35810 & -1.94001 \\ \mathrm{H} & -.29029 & -.75869 & 1.11687 \\ \mathrm{H} & 2.00028 & -.65632 & 1.92424 \\ \mathrm{H} & -.54368 & -.62176 & 3.54426 \\ \mathrm{H} & -1.36135 & .63909 & 5.51993 \\ \mathrm{H} & -1.89017 & 3.06109 & 5.31721 \\ \mathrm{H} & -1.61942 & 4.20588 & 3.12580 \\ \mathrm{H} & -.80876 & 2.91113 & 1.14144 \\ \mathrm{H} & 1.66263 & 2.06586 & -2.89847 \\ \mathrm{H} & 2.40813 & 2.91785 & -1.49708 \\ \mathrm{H} & .82709 & 2.08178 & -1.29591 \\ \mathrm{H} & 3.88437 & -2.18497 & -1.86548 \\ \mathrm{H} & 4.71473 & -.60234 & -2.03557 \\ \mathrm{H} & 5.37928 & -1.85199 & -.94146 \\ \mathrm{H} & -.10913 & -2.34752 & -1.95627 \\ \mathrm{H} & .75768 & -1.12048 & -2.92649 \\ \mathrm{H} & -. .34362 & -.58703 & -1.59340 \\ \mathrm{C} & 2.33044 & 1.45998 & 1.74436 \\ \mathrm{O} & 2.51950 & 1.42297 & 3.08533 \\ \mathrm{O} & 2.53090 & 2.46045 & 1.07193 \\ \mathrm{C} & 2.87436 & 2.67722 & 3.68431 \\ \mathrm{H} & 3.00240 & 2.46533 & 4.74643 \\ \mathrm{H} & 3.80215 & 3.06394 & 3.25390 \\ \mathrm{H} & 2.07612 & 3.40875 & 3.53467 \\ & & & \\ & & \end{array}$

\section{Cis TS1 (B3LYP 6-31G*(THF))}

$\mathrm{E}(\mathrm{B} 3 \mathrm{LYP} 6-31 \mathrm{G} *(\mathrm{THF}))=$ $-1299.50757$

$\begin{array}{llll}\mathrm{O} & -.36412 & .76644 & -.13368 \\ \mathrm{C} & -.14151 & .29709 & 1.04485 \\ \mathrm{C} & 1.77432 & .15996 & 1.25961 \\ \mathrm{P} & 2.27378 & -.24570 & -.39813 \\ \mathrm{C} & -.62592 & 1.09380 & 2.24181 \\ \mathrm{C} & -.86246 & .47855 & 3.47725 \\ \mathrm{C} & -1.34946 & 1.21707 & 4.55787 \\ \mathrm{C} & -1.60245 & 2.58535 & 4.41304 \\ \mathrm{C} & -1.37272 & 3.20568 & 3.18044 \\ \mathrm{C} & -.89265 & 2.46122 & 2.10147 \\ \mathrm{O} & 2.45144 & .88518 & -1.47675\end{array}$

$\begin{array}{cccc}\mathrm{C} & 1.67967 & 2.08250 & -1.81001 \\ \mathrm{O} & 3.74002 & -.86160 & -.25928 \\ \mathrm{C} & 4.45289 & -1.40775 & -1.40503 \\ \mathrm{O} & 1.43175 & -1.45969 & -.98151 \\ \mathrm{C} & .44756 & -1.37831 & -2.05415 \\ \mathrm{H} & -.30435 & -.78689 & 1.21513 \\ \mathrm{H} & 1.97763 & -.69219 & 1.91055 \\ \mathrm{H} & -.67337 & -.58846 & 3.58946 \\ \mathrm{H} & -1.53971 & .72667 & 5.50998 \\ \mathrm{H} & -1.98629 & 3.16163 & 5.25208 \\ \mathrm{H} & -1.58043 & 4.26676 & 3.05761 \\ \mathrm{H} & -.73458 & 2.92282 & 1.13186 \\ \mathrm{H} & 1.40496 & 1.98528 & -2.86237 \\ \mathrm{H} & 2.34671 & 2.93089 & -1.66153 \\ \mathrm{H} & .80090 & 2.13552 & -1.17199 \\ \mathrm{H} & 3.88016 & -2.22819 & -1.84427 \\ \mathrm{H} & 4.62769 & -.62164 & -2.14290 \\ \mathrm{H} & 5.40288 & -1.77775 & -1.01855 \\ \mathrm{H} & .08236 & -2.39763 & -2.18296 \\ \mathrm{H} & .93421 & -1.03665 & -2.97124 \\ \mathrm{H} & -.34473 & -.69899 & -1.74135 \\ \mathrm{C} & 2.31075 & 1.43034 & 1.77039 \\ \mathrm{O} & 2.48958 & 1.38985 & 3.11145 \\ \mathrm{O} & 2.50780 & 2.44245 & 1.10750 \\ \mathrm{C} & 2.83453 & 2.64272 & 3.73392 \\ \mathrm{H} & 2.98502 & 2.41469 & 4.78992 \\ \mathrm{H} & 3.75035 & 3.05324 & 3.30090 \\ \mathrm{H} & 2.02156 & 3.36323 & 3.61285\end{array}$

Trans TS1 (B3LYP 6-31G*)

$\mathrm{E}($ B3LYP 6-31G $*)=-1299.48750$

$\mathrm{E}\left(\right.$ B3LYP $\left.6-31 \mathrm{G}^{*}(\mathrm{THF})\right)=$ $-1299.50808$

$\begin{array}{lccc}\mathrm{O} & -.16370 & -.06350 & -.14027 \\ \mathrm{C} & .10341 & -.04008 & 1.12506 \\ \mathrm{C} & 1.99050 & -.03563 & 1.19502 \\ \mathrm{P} & 2.33129 & -1.21672 & -.08066 \\ \mathrm{C} & -. .31792 & 1.17341 & 1.93304 \\ \mathrm{C} & -.37508 & 1.13465 & 3.33170 \\ \mathrm{C} & -.77206 & 2.25868 & 4.05672 \\ \mathrm{C} & -1.12055 & 3.43518 & 3.38824 \\ \mathrm{C} & -1.07964 & 3.47576 & 1.99232 \\ \mathrm{C} & -.68414 & 2.34959 & 1.27015 \\ \mathrm{O} & 3.81720 & -1.73729 & .19398 \\ \mathrm{C} & 4.40914 & -2.78874 & -.59676 \\ \mathrm{O} & 1.49873 & -2.56406 & -.11013 \\ \mathrm{C} & .31083 & -2.81652 & -.91711 \\ \mathrm{O} & 2.34915 & -.63370 & -1.56162 \\ \mathrm{C} & 1.98275 & .71462 & -1.96578 \\ \mathrm{H} & -.05583 & -.96750 & 1.71350 \\ \mathrm{H} & 2.21536 & .97029 & .84321 \\ \mathrm{H} & -.11661 & .21367 & 3.85178 \\ \mathrm{H} & -.81634 & 2.21652 & 5.14227 \\ \mathrm{H} & -1.43207 & 4.31129 & 3.95164 \\ \mathrm{H} & -1.36505 & 4.38450 & 1.46731 \\ \mathrm{H} & -.67384 & 2.35229 & .18437\end{array}$




$\begin{array}{cccc}\mathrm{H} & 4.45091 & -2.49334 & -1.64946 \\ \mathrm{H} & 3.83695 & -3.71202 & -.48273 \\ \mathrm{H} & 5.41869 & -2.92078 & -.20742 \\ \mathrm{H} & .61075 & -2.91369 & -1.96368 \\ \mathrm{H} & -. .38246 & -1.98421 & -.77961 \\ \mathrm{H} & -.08402 & -3.76248 & -.54651 \\ \mathrm{H} & 1.93746 & .68536 & -3.05518 \\ \mathrm{H} & 2.76171 & 1.41431 & -1.64841 \\ \mathrm{H} & 1.01247 & .95389 & -1.52853 \\ \mathrm{C} & 2.55850 & -.34858 & 2.52028 \\ \mathrm{O} & 2.98859 & .77634 & 3.14510 \\ \mathrm{O} & 2.55279 & -1.44885 & 3.04658 \\ \mathrm{C} & 3.40127 & .59975 & 4.50641 \\ \mathrm{H} & 3.70170 & 1.59002 & 4.85089 \\ \mathrm{H} & 2.57549 & .21911 & 5.11383 \\ \mathrm{H} & 4.23872 & -.10046 & 4.56934\end{array}$

Trans TS1 (B3LYP 6-31G*(THF))

$\begin{array}{lccc}\text { E(B3LYP 6-31G*(THF) })= \\ -1299.51046 & & \\ \text { O } & -.47082 & -.14845 & .04425 \\ \text { C } & -.00216 & -.10839 & 1.25460 \\ \text { C } & 1.83294 & .07182 & 1.06568 \\ \text { P } & 2.15665 & -1.10835 & -.22999 \\ \text { C } & -.43863 & 1.04614 & 2.14646 \\ \text { C } & -.42497 & .93579 & 3.54290 \\ \text { C } & -.86951 & 1.99022 & 4.34484 \\ \text { C } & -1.33853 & 3.16830 & 3.75594 \\ \text { C } & -1.36475 & 3.28205 & 2.36182 \\ \text { C } & -.92126 & 2.22498 & 1.56534 \\ \text { O } & 3.70764 & -1.46379 & -.12681 \\ \text { C } & 4.30793 & -2.51132 & -.94133 \\ \text { O } & 1.46565 & -2.52279 & -.10496 \\ \text { C } & .21383 & -2.95083 & -.72863 \\ \text { O } & 1.88177 & -.57415 & -1.69795 \\ \text { C } & 1.52160 & .79595 & -2.05290 \\ \text { H } & -.00050 & -1.05122 & 1.84299 \\ \text { H } & 1.93466 & 1.08692 & .68354 \\ \text { H } & -.08131 & .01122 & 4.00462 \\ \text { H } & -.86199 & 1.88915 & 5.42781 \\ \text { H } & -1.69163 & 3.98766 & 4.37824 \\ \text { H } & -1.74164 & 4.19104 & 1.89722 \\ \text { H } & -.96520 & 2.28776 & .48177 \\ \text { H } & 4.15218 & -2.29811 & -2.00201 \\ \text { H } & 3.87778 & -3.47868 & -.67482 \\ \text { H } & 5.37266 & -2.49310 & -.70841 \\ \text { H } & .40229 & -3.16043 & -1.78366 \\ \text { H } & -.52512 & -2.15944 & -.59905 \\ \text { H } & -.06580 & -3.86520 & -.20463 \\ \text { H } & 2.34457 & 1.47263 & -1.80760 \\ \text { H } & .60516 & 1.05276 & -1.52214 \\ \text { H } & 1.36342 & .77993 & -3.13162 \\ \text { C } & 2.55158 & -.16124 & 2.33414 \\ \text { O } & 2.90433 & .99824 & 2.92866 \\ \text { O } & 2.70738 & -1.25750 & 2.85564 \\ \text { C } & 3.41683 & .89083 & 4.27186 \\ & & & \\ & & \end{array}$

$$
\begin{array}{cccc}
\mathrm{H} & 3.63443 & 1.91345 & 4.58154 \\
\mathrm{H} & 2.66865 & .44495 & 4.93248 \\
\mathrm{H} & 4.32567 & .28417 & 4.29199
\end{array}
$$

\section{Cis OP1 (B3LYP 6-31G*)}

E(B3LYP 6-31G*) $=-1299.53288$

$\mathrm{E}\left(\mathrm{B} 3 \mathrm{LYP} 6-31 \mathrm{G}^{*}(\mathrm{THF})\right)=$ $-1299.54652$

$\begin{array}{lccc}\mathrm{O} & .44097 & 1.10609 & -.46695 \\ \mathrm{C} & .34822 & .61306 & .87217 \\ \mathrm{C} & 1.86183 & .67135 & 1.20996 \\ \mathrm{P} & 2.18975 & .96331 & -.60908 \\ \mathrm{C} & -.61920 & 1.38599 & 1.74286 \\ \mathrm{C} & -.84093 & .96390 & 3.06077 \\ \mathrm{C} & -1.73181 & 1.65014 & 3.88461 \\ \mathrm{C} & -2.42076 & 2.76299 & 3.39575 \\ \mathrm{C} & -2.21187 & 3.17950 & 2.08075 \\ \mathrm{C} & -1.31508 & 2.49514 & 1.25766 \\ \mathrm{O} & 2.35942 & 2.36765 & -1.41105 \\ \mathrm{C} & 1.34844 & 3.37291 & -1.57899 \\ \mathrm{O} & 2.15181 & -.31479 & -1.62772 \\ \mathrm{C} & 1.04664 & -.59770 & -2.49495 \\ \mathrm{O} & 3.82679 & .80649 & -.35430 \\ \mathrm{C} & 4.79128 & .94624 & -1.39393 \\ \mathrm{H} & .03684 & -.44354 & .86475 \\ \mathrm{H} & 2.30231 & -.25285 & 1.58580 \\ \mathrm{H} & -.31201 & .09355 & 3.44542 \\ \mathrm{H} & -1.89124 & 1.31441 & 4.90609 \\ \mathrm{H} & -3.11771 & 3.29778 & 4.03562 \\ \mathrm{H} & -2.74760 & 4.04169 & 1.69186 \\ \mathrm{H} & -1.14968 & 2.81183 & .23430 \\ \mathrm{H} & .47886 & 2.98377 & -2.11271 \\ \mathrm{H} & 1.82292 & 4.16161 & -2.16850 \\ \mathrm{H} & 1.04670 & 3.76565 & -.60642 \\ \mathrm{H} & .13498 & -.79953 & -1.92935 \\ \mathrm{H} & .86382 & .23566 & -3.18124 \\ \mathrm{H} & 1.34097 & -1.47968 & -3.06857 \\ \mathrm{H} & 5.76027 & .67735 & -.96327 \\ \mathrm{H} & 4.82874 & 1.97851 & -1.75715 \\ \mathrm{H} & 4.57141 & .27321 & -2.22995 \\ \mathrm{C} & 2.30784 & 1.85005 & 2.04301 \\ \mathrm{O} & 2.97442 & 1.45055 & 3.14387 \\ \mathrm{O} & 2.09502 & 3.01385 & 1.77022 \\ \mathrm{C} & 3.45596 & 2.51174 & 3.98678 \\ \mathrm{H} & 3.97724 & 2.01779 & 4.80714 \\ \mathrm{H} & 4.13780 & 3.15979 & 3.43025 \\ \mathrm{H} & 2.62160 & 3.10954 & 4.36231\end{array}$

Trans OP1 (B3LYP 6-31G*)

$\mathrm{E}\left(\mathrm{B} 3 \mathrm{LYP} 6-31 \mathrm{G}^{*}\right)=-1299.53724$

$\mathrm{E}(\mathrm{B} 3 \mathrm{LYP} 6-31 \mathrm{G} *(\mathrm{THF}))=$ $-1299.55145$

$\begin{array}{llll}\mathrm{O} & .41629 & .86217 & -.79585 \\ \mathrm{C} & .32133 & .50203 & .59705\end{array}$

$\begin{array}{lccc}\text { C } & 1.81266 & .66631 & .94378 \\ \text { P } & 2.14484 & 1.08232 & -.86288 \\ \text { C } & -.63636 & 1.38886 & 1.36102 \\ \text { C } & -1.60570 & .82217 & 2.19389 \\ \text { C } & -2.48025 & 1.63055 & 2.92401 \\ \text { C } & -2.39579 & 3.01910 & 2.82283 \\ \text { C } & -1.43218 & 3.59447 & 1.98950 \\ \text { C } & -.55899 & 2.78556 & 1.26481 \\ \text { O } & 3.75433 & 1.32550 & -.51594 \\ \text { C } & 4.72919 & 1.63223 & -1.50998 \\ \text { O } & 2.44304 & -.09744 & -1.94286 \\ \text { C } & 1.49237 & -1.08016 & -2.38366 \\ \text { O } & 2.03690 & 2.54780 & -1.58863 \\ \text { C } & .93317 & 2.94746 & -2.41260 \\ \text { H } & .01188 & -.54512 & .68997 \\ \text { H } & 2.04712 & 1.51028 & 1.59479 \\ \text { H } & -1.67873 & -.26074 & 2.26868 \\ \text { H } & -3.22947 & 1.17428 & 3.56566 \\ \text { H } & -3.07722 & 3.65089 & 3.38641 \\ \text { H } & -1.36245 & 4.67588 & 1.90435 \\ \text { H } & .18583 & 3.23942 & .61631 \\ \text { H } & 4.49636 & 2.57668 & -2.01216 \\ \text { H } & 4.79725 & .83009 & -2.25277 \\ \text { H } & 5.68739 & 1.72551 & -.99075 \\ \text { H } & 1.27823 & -1.78038 & -1.57209 \\ \text { H } & 1.98198 & -1.60868 & -3.20503 \\ \text { H } & .56580 & -.62051 & -2.73106 \\ \text { H } & 1.16817 & 3.95965 & -2.75064 \\ \text { H } & -.00371 & 2.93904 & -1.85377 \\ \text { H } & .83950 & 2.28899 & -3.28284 \\ \text { C } & 2.52824 & -.57951 & 1.39730 \\ \text { O } & 3.50059 & -.30449 & 2.28434 \\ \text { O } & 2.27031 & -1.70099 & 1.00351 \\ \text { C } & 4.29297 & -1.43032 & 2.69757 \\ \text { H } & 3.66732 & -2.18308 & 3.18426 \\ \text { H } & 4.78965 & -1.88234 & 1.83518 \\ \text { H } & 5.02635 & -1.02838 & 3.39692 \\ & & & \end{array}$

Cis OP2 (B3LYP 6-31G*)

$\mathrm{E}\left(\right.$ B3LYP $\left.6-31 \mathrm{G}^{*}\right)=-1299.52913$ E(B3LYP 6-31G*(THF)) = $-1299.54308$

$\begin{array}{lccl}\mathrm{O} & .34687 & 1.06152 & -.33217 \\ \mathrm{C} & .21214 & .67331 & 1.06498 \\ \mathrm{C} & 1.71864 & .70387 & 1.41523 \\ \mathrm{P} & 2.00848 & .89856 & -.47823 \\ \mathrm{C} & -.78252 & 1.52643 & 1.81528 \\ \mathrm{C} & -1.36313 & 1.00888 & 2.97940 \\ \mathrm{C} & -2.28045 & 1.76169 & 3.71261 \\ \mathrm{C} & -2.63986 & 3.03821 & 3.27687 \\ \mathrm{C} & -2.07418 & 3.55313 & 2.10864 \\ \mathrm{C} & -1.14791 & 2.80448 & 1.38313 \\ \mathrm{O} & 3.22367 & 1.96862 & -.25201 \\ \mathrm{C} & 3.80334 & 2.85195 & -1.22190 \\ \mathrm{O} & 2.53803 & -.64922 & -.61333 \\ \mathrm{C} & 3.07286 & -1.21445 & -1.81752\end{array}$




$\begin{array}{cccc}\mathrm{O} & 1.93685 & 1.26387 & -2.10947 \\ \mathrm{C} & .80886 & .99526 & -2.93711 \\ \mathrm{H} & -.13399 & -.36692 & 1.09179 \\ \mathrm{H} & 2.10267 & -.23533 & 1.81153 \\ \mathrm{H} & -1.09621 & .00810 & 3.31467 \\ \mathrm{H} & -2.71988 & 1.34754 & 4.61639 \\ \mathrm{H} & -3.35903 & 3.62586 & 3.84153 \\ \mathrm{H} & -2.35061 & 4.54578 & 1.76273 \\ \mathrm{H} & -.70080 & 3.20881 & .48224 \\ \mathrm{H} & 4.29716 & 2.29609 & -2.02270 \\ \mathrm{H} & 4.53805 & 3.44457 & -.67109 \\ \mathrm{H} & 3.04688 & 3.50908 & -1.65498 \\ \mathrm{H} & 2.29308 & -1.37675 & -2.56694 \\ \mathrm{H} & 3.85574 & -.58224 & -2.24401 \\ \mathrm{H} & 3.49988 & -2.17743 & -1.52735 \\ \mathrm{H} & 1.14517 & 1.12531 & -3.97077 \\ \mathrm{H} & -.00704 & 1.69249 & -2.72669 \\ \mathrm{H} & .43065 & -.02590 & -2.81031 \\ \mathrm{C} & 2.20847 & 1.86021 & 2.23984 \\ \mathrm{O} & 3.22746 & 1.47235 & 3.04654 \\ \mathrm{O} & 1.79164 & 2.99820 & 2.19860 \\ \mathrm{C} & 3.83052 & 2.52394 & 3.81238 \\ \mathrm{H} & 4.62826 & 2.04940 & 4.38549 \\ \mathrm{H} & 4.23833 & 3.29480 & 3.15242 \\ \mathrm{H} & 3.09845 & 2.98453 & 4.48147\end{array}$

Trans OP2 (B3LYP 6-31G*)

$\mathrm{E}($ B3LYP 6-31G*) $=-1299.53653$

$\mathrm{E}(\mathrm{B} 3 \mathrm{LYP} 6-31 \mathrm{G} *(\mathrm{THF}))=$ $-1299.55042$

$\begin{array}{llll}\mathrm{O} & .40759 & .81559 & -.62513 \\ \mathrm{C} & .44945 & .27732 & .73063 \\ \mathrm{C} & 1.95271 & .47664 & .95958 \\ \mathrm{P} & 2.05099 & .99683 & -.88535 \\ \mathrm{C} & -.48948 & .99347 & 1.66933 \\ \mathrm{C} & -.84912 & .36358 & 2.86835 \\ \mathrm{C} & -1.67554 & 1.00935 & 3.78689 \\ \mathrm{C} & -2.15958 & 2.29109 & 3.51346 \\ \mathrm{C} & -1.81148 & 2.91871 & 2.31665 \\ \mathrm{C} & -.97946 & 2.27473 & 1.39845 \\ \mathrm{O} & 2.92778 & 2.32645 & -.51422 \\ \mathrm{C} & 3.28949 & 3.39332 & -1.40484 \\ \mathrm{O} & 2.93434 & -.32686 & -1.28580 \\ \mathrm{C} & 3.45441 & -.61983 & -2.58897 \\ \mathrm{O} & 1.78361 & 1.53537 & -2.44984 \\ \mathrm{C} & .69778 & 1.09025 & -3.25739 \\ \mathrm{H} & .20796 & -.78869 & .69210 \\ \mathrm{H} & 2.18353 & 1.33621 & 1.59105 \\ \mathrm{H} & -.47593 & -.63658 & 3.07885 \\ \mathrm{H} & -1.94682 & .50958 & 4.71320 \\ \mathrm{H} & -2.80804 & 2.79370 & 4.22627 \\ \mathrm{H} & -2.19049 & 3.91282 & 2.09333 \\ \mathrm{H} & -.71945 & 2.75809 & .46207 \\ \mathrm{H} & 2.40348 & 3.88644 & -1.80940 \\ \mathrm{H} & 3.90567 & 3.03105 & -2.23153 \\ \mathrm{H} & 3.86460 & 4.09562 & -.79604\end{array}$

\begin{abstract}
$\begin{array}{llll}\mathrm{H} & 3.99153 & .23353 & -3.00929\end{array}$
$\begin{array}{lllll}\mathrm{H} & 2.66020 & -.92150 & -3.27727\end{array}$

H $\quad 4.14408-1.45550 \quad-2.44893$

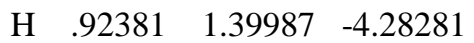

$\begin{array}{lllll}\mathrm{H} & -.24251 & 1.54694 & -2.93529\end{array}$

H $\quad .57591 \quad .00076 \quad-3.23379$

$\begin{array}{llll}\text { C } & 2.71083 & -.72946 & 1.42967\end{array}$

$\begin{array}{llll}\text { O } & 3.99040 & -.41075 & 1.72677\end{array}$

$\begin{array}{llll}\text { O } & 2.25870 & -1.85127 & 1.54546\end{array}$

$\begin{array}{llll}\text { C } & 4.81581 & -1.51129 & 2.13181\end{array}$

$\begin{array}{llll}\text { H } & 5.80082 & -1.08369 & 2.32352\end{array}$

H $4.41924 \quad-1.98152 \quad 3.03593$

$\begin{array}{llll}\mathrm{H} & 4.87141 & -2.26221 & 1.33881\end{array}$
\end{abstract}

\section{Cis TS2 (B3LYP 6-31G*)}

$\mathrm{E}\left(\mathrm{B} 3 \mathrm{LYP} 6-31 \mathrm{G}^{*}\right)=-1299.50034$

$\mathrm{E}(\mathrm{B} 3 \mathrm{LYP} 6-31 \mathrm{G} *(\mathrm{THF}))=$ $-1299.51604$

$\begin{array}{cccc}\mathrm{C} & .06326 & -.02517 & -.05883 \\ \mathrm{C} & -.20873 & -.19915 & 1.36621 \\ \mathrm{P} & 2.42919 & -.06310 & 1.10280 \\ \mathrm{O} & 1.64377 & .08368 & -.25161 \\ \mathrm{C} & -.51551 & 1.13064 & -.85288 \\ \mathrm{C} & -1.86724 & 1.45109 & -.67098 \\ \mathrm{C} & -2.47516 & 2.43350 & -1.44969 \\ \mathrm{C} & -1.74117 & 3.11710 & -2.42166 \\ \mathrm{C} & -. .39620 & 2.80221 & -2.61095 \\ \mathrm{C} & .21111 & 1.81063 & -1.83761 \\ \mathrm{O} & 2.45164 & 1.08062 & 2.19512 \\ \mathrm{C} & 2.55126 & 2.48661 & 1.82058 \\ \mathrm{O} & 2.45444 & -1.46942 & 1.83967 \\ \mathrm{C} & 1.96208 & -1.70874 & 3.18125 \\ \mathrm{O} & 3.94065 & .03913 & .55271 \\ \mathrm{C} & 4.41980 & -.83555 & -.48273 \\ \mathrm{H} & -.10976 & -.95970 & -.59908 \\ \mathrm{H} & -.50286 & -1.19463 & 1.68093 \\ \mathrm{H} & -2.43715 & .93147 & .09325 \\ \mathrm{H} & -3.52293 & 2.67334 & -1.28865 \\ \mathrm{H} & -2.21381 & 3.88853 & -3.02405 \\ \mathrm{H} & .18685 & 3.32540 & -3.36494 \\ \mathrm{H} & 1.25601 & 1.56904 & -1.99680 \\ \mathrm{H} & 3.42992 & 2.63644 & 1.18865 \\ \mathrm{H} & 2.66805 & 3.01941 & 2.76368 \\ \mathrm{H} & 1.62398 & 2.78687 & 1.33418 \\ \mathrm{H} & 2.71689 & -1.37447 & 3.89705 \\ \mathrm{H} & 1.01757 & -1.18876 & 3.34228 \\ \mathrm{H} & 1.82499 & -2.78812 & 3.25445 \\ \mathrm{H} & 5.45716 & -.55076 & -.66534 \\ \mathrm{H} & 3.83113 & -.70991 & -1.39533 \\ \mathrm{H} & 4.38012 & -1.87788 & -.14996 \\ \mathrm{C} & -.55034 & .87640 & 2.24159 \\ \mathrm{O} & -.98415 & .39601 & 3.47105 \\ \mathrm{O} & -.44745 & 2.08865 & 2.05088 \\ \mathrm{C} & -1.23061 & 1.40034 & 4.45226 \\ \mathrm{H} & -1.55209 & .86641 & 5.34970 \\ \mathrm{H} & -.32632 & 1.98114 & 4.66468 \\ & & & \end{array}$

$\begin{array}{llll}\mathrm{H} & -2.01253 & 2.09312 & 4.12621\end{array}$

\section{Trans TS2 (B3LYP 6-31G*)}

$\mathrm{E}\left(\mathrm{B} 3 \mathrm{LYP} 6-31 \mathrm{G}^{*}\right)=-1299.50149$
$\mathrm{E}\left(\mathrm{B} 3 \mathrm{LYP} 6-31 \mathrm{G}^{*}(\mathrm{THF})\right)=$
-1299.52120

$\begin{array}{llll}\text { C } & -.17865 & .08984 & .07097\end{array}$

$\begin{array}{llll}\text { C } & -.25642 & .01191 & 1.50935\end{array}$

$\begin{array}{llll}\text { P } & 2.24766 & -.06176 & 1.05059\end{array}$

$\begin{array}{llll}\mathrm{O} & 1.49482 & .01850 & -.30804\end{array}$

$\begin{array}{llll}\text { C } & -.59680 & 1.32649 & -.65058\end{array}$

$\begin{array}{llll}\text { C } & -1.33991 & 1.21520 & -1.83159\end{array}$

$\begin{array}{llll}\text { C } & -1.76916 & 2.35538 & -2.51314\end{array}$

$\begin{array}{llll}\text { C } & -1.44606 & 3.62212 & -2.02714\end{array}$

$\begin{array}{llll}\text { C } & -.69519 & 3.74292 & -.85353\end{array}$

$\begin{array}{llll}\text { C } & -.27661 & 2.60534 & -.16914\end{array}$

$\begin{array}{llll}\text { O } & 2.22655 & 1.12955 & 2.12686\end{array}$

$\begin{array}{llll}\text { C } & 3.35710 & 2.00315 & 2.38760\end{array}$

$\begin{array}{llll}\text { O } & 2.27696 & -1.50941 & 1.73000\end{array}$

$\begin{array}{llll}\text { C } & 2.25073 & -1.70924 & 3.15717\end{array}$

$\begin{array}{lllll}\text { O } & 3.79416 & .10129 & .57596\end{array}$

$\begin{array}{llll}\text { C } & 4.32802 & -.74987 & -.45042\end{array}$

$\begin{array}{llll}\mathrm{H} & -.47657 & -.82915 & -.42919\end{array}$

$\begin{array}{llll}\mathrm{H} & -.45416 & .91458 & 2.07499\end{array}$

$\begin{array}{lllll}\mathrm{H} & -1.58415 & .22781 & -2.21588\end{array}$

$\begin{array}{llll}\mathrm{H} & -2.35007 & 2.25191 & -3.42568\end{array}$

$\begin{array}{lllll}\mathrm{H} & -1.77371 & 4.51156 & -2.55867\end{array}$

$\begin{array}{llll}\mathrm{H} & -.43703 & 4.72754 & -.47221\end{array}$

$\begin{array}{llll}\mathrm{H} & .31065 & 2.70396 & .73937\end{array}$

$\begin{array}{llll}\mathrm{H} & 3.61791 & 2.57123 & 1.49326\end{array}$

$\begin{array}{llll}\mathrm{H} & 4.22302 & 1.43133 & 2.72521\end{array}$

$\begin{array}{llll}\mathrm{H} & 3.01416 & 2.67375 & 3.17677\end{array}$

$\begin{array}{llll}\mathrm{H} & 1.42800 & -1.14949 & 3.60471\end{array}$

$\begin{array}{llll}\mathrm{H} & 3.20728 & -1.40865 & 3.59725\end{array}$

$\begin{array}{llll}\mathrm{H} & 2.09557 & -2.77891 & 3.29909\end{array}$

$\begin{array}{lllll}\mathrm{H} & 5.37686 & -.46942 & -.56538\end{array}$

$\begin{array}{lllll}\mathrm{H} & 3.79283 & -.59434 & -1.39056\end{array}$

$\begin{array}{llll}\mathrm{H} & 4.25982 & -1.80098 & -.15174\end{array}$

$\begin{array}{llll}\text { C } & -.74202 & -1.22519 & 2.07321\end{array}$

$\begin{array}{llll}\text { O } & -1.05777 & -1.08114 & 3.41238\end{array}$

$\begin{array}{llll}\mathrm{O} & -.85422 & -2.30908 & 1.51179\end{array}$

$\begin{array}{llll}\text { C } & -1.55728 & -2.26159 & 4.04022\end{array}$

$\begin{array}{llll}\mathrm{H} & -1.76546 & -1.98156 & 5.07531\end{array}$

$\begin{array}{llll}\mathrm{H} & -2.47228 & -2.61214 & 3.55294\end{array}$

$\begin{array}{llll}\mathrm{H} & -.82254 & -3.07317 & 4.00926\end{array}$

\section{J. $\mathbf{P h}_{3} \mathbf{P C H}_{2}+\mathrm{CH}_{2} \mathrm{O}$}

\section{TS1 (B3LYP 6-31G*)}

$\mathrm{E}(\mathrm{B} 3 \mathrm{LYP}$ 6-31G*) $=-1190.05843$

$\begin{array}{lrcc}\mathrm{O} & 1.11017 & 1.41164 & 2.61952 \\ \mathrm{C} & 1.67100 & 1.16012 & 1.52450 \\ \mathrm{P} & -1.00748 & .14676 & -.02227\end{array}$ 


\begin{tabular}{|c|c|c|c|}
\hline & & & \\
\hline & -1.77350 & & \\
\hline & 02 & & \\
\hline & & & \\
\hline & & & \\
\hline & & & \\
\hline & -3 & & \\
\hline & -1. & & \\
\hline & $-1 . \varsigma$ & & \\
\hline & & & \\
\hline & & & \\
\hline & & & \\
\hline & & & \\
\hline & & & \\
\hline & & & \\
\hline & -2 & & \\
\hline & -3.4 & -1 . & \\
\hline & -4.0 & -1 & \\
\hline & -3.2 & -.8 & 1.3 \\
\hline & 1.7 & & \\
\hline & 1.2 & -.1 & -.6 \\
\hline & -.1 & -2. & \\
\hline & -1. & & \\
\hline & & & \\
\hline & & & \\
\hline & & & \\
\hline & & & \\
\hline & & & \\
\hline & & & \\
\hline & & 4.8 & 1.3 \\
\hline & -.6 & 2.44 & 1.6 \\
\hline & -.382 & . 161 & 2.81 \\
\hline & -1.66072 & -.52 & 4.8 \\
\hline & -3.9 & -1.3 & \\
\hline & -5.02 & -1.5 & \\
\hline & & & \\
\hline & & & \\
\hline & & -1.1 & \\
\hline
\end{tabular}

\section{TS1b (B3L YP 6-31G*)}

$\begin{array}{lccc}\mathrm{E}(\mathrm{B} 3 L Y P & \left.6-31 \mathrm{G}^{*}\right)=-1190.05778 \\ \mathrm{O} & 4.08528 & .32024 & .96277 \\ \mathrm{C} & 3.43371 & -.03234 & 1.92718 \\ \mathrm{P} & -.18954 & -.09281 & .35848 \\ \mathrm{C} & .65476 & -.13253 & 1.83648 \\ \mathrm{H} & .56000 & .78198 & 2.41913 \\ \mathrm{H} & 3.25160 & -1.09758 & 2.16463 \\ \mathrm{C} & -2.04040 & -.30591 & .36149 \\ \mathrm{C} & -2.78337 & -.81539 & -.71470 \\ \mathrm{C} & -4.16732 & -.96209 & -.60723 \\ \mathrm{C} & -4.82286 & -.60302 & .57331 \\ \mathrm{C} & -4.09075 & -.10056 & 1.65035 \\ \mathrm{C} & -2.70638 & .04058 & 1.54524 \\ \mathrm{C} & .10976 & 1.51562 & -.45832 \\ \mathrm{C} & .39862 & -1.40667 & -.77712 \\ \text { C } & 1.44433 & 1.92658 & -.61981\end{array}$

$\begin{array}{cccc}\text { C } & 1.72424 & 3.15421 & -1.21942 \\ \text { C } & .68391 & 3.97978 & -1.65449 \\ \text { C } & -.64088 & 3.57449 & -1.49529 \\ \text { C } & -.93120 & 2.34457 & -.90005 \\ \text { C } & .85210 & -2.62343 & -.24827 \\ \text { C } & 1.29653 & -3.63688 & -1.09619 \\ \text { C } & 1.29936 & -3.44450 & -2.47985 \\ \text { C } & .86028 & -2.23240 & -3.01316 \\ \text { C } & .41200 & -1.21575 & -2.16643 \\ \text { H } & 3.04310 & .68104 & 2.67489 \\ \text { H } & .55982 & -1.04723 & 2.42064 \\ \text { H } & -2.28304 & -1.11079 & -1.63225 \\ \text { H } & -4.73392 & -1.36426 & -1.44304 \\ \text { H } & -5.89960 & -.72469 & .65605 \\ \text { H } & -4.59404 & .17002 & 2.57491 \\ \text { H } & -2.12456 & .40120 & 2.39060 \\ \text { H } & 2.25092 & 1.27798 & -.29311 \\ \text { H } & 2.75796 & 3.46376 & -1.34543 \\ \text { H } & .90636 & 4.93719 & -2.11860 \\ \text { H } & -1.45186 & 4.21486 & -1.83179 \\ \text { H } & -1.96454 & 2.03754 & -.77697 \\ \text { H } & .87618 & -2.77330 & .82696 \\ \text { H } & 1.64998 & -4.57451 & -.67595 \\ \text { H } & 1.65281 & -4.23320 & -3.13849 \\ \text { H } & .87475 & -2.07135 & -4.08777 \\ \text { H } & .08926 & -.26788 & -2.58847\end{array}$

\section{K. $(\mathrm{MeO})_{3} \mathrm{PCH}_{2}$ $\mathrm{CH}_{2} \mathrm{O}$}

\section{TS1 (B3LYP 6-31G*)}

$\mathrm{E}\left(\mathrm{B} 3 \mathrm{LYP} 6-31 \mathrm{G}^{*}\right)=-840.54850$

$\begin{array}{cccc}\mathrm{O} & -.63812 & 1.02627 & -.39010 \\ \mathrm{C} & -.05812 & 1.14077 & .72079 \\ \mathrm{C} & 1.42708 & -.26016 & 1.06490 \\ \mathrm{P} & 2.40975 & -.42312 & -.32580 \\ \mathrm{H} & .62957 & 1.99182 & .90338 \\ \mathrm{O} & 2.85391 & .97024 & -.97340 \\ \mathrm{C} & 1.99407 & 1.72731 & -1.87497 \\ \mathrm{O} & 3.83977 & -1.10525 & -.07329 \\ \mathrm{C} & 4.82987 & -1.22378 & -1.11922 \\ \mathrm{O} & 1.74076 & -1.30086 & -1.47914 \\ \mathrm{C} & .31854 & -1.61405 & -1.56875 \\ \mathrm{H} & -.56925 & .82600 & 1.65266 \\ \mathrm{H} & .92892 & -1.19651 & 1.31864 \\ \mathrm{H} & 2.09621 & 1.31344 & -2.88226 \\ \mathrm{H} & 2.37921 & 2.74739 & -1.85437 \\ \mathrm{H} & .95002 & 1.69243 & -1.54348 \\ \mathrm{H} & 4.44780 & -1.84971 & -1.93056 \\ \mathrm{H} & 5.09948 & -.23515 & -1.49798 \\ \mathrm{H} & 5.69643 & -1.69990 & -.65888 \\ \mathrm{H} & .14364 & -2.56661 & -1.05996 \\ \mathrm{H} & .11166 & -1.72633 & -2.63437 \\ \mathrm{H} & -.28387 & -.80512 & -1.14264 \\ \mathrm{H} & 1.97525 & .16093 & 1.90840\end{array}$

TS1b (B3LYP 6-31G*)

$\mathrm{E}\left(\mathrm{B} 3 \mathrm{LYP} 6-31 \mathrm{G}^{*}\right)=-840.54755$

$\begin{array}{lllll}\mathrm{O} & -.60610 & 1.24368 & -.27549\end{array}$

$\begin{array}{llll}\text { C } & -.30513 & .68167 & .80922\end{array}$

$\begin{array}{llll}\text { C } & 1.70406 & .35035 & 1.09321\end{array}$

$\begin{array}{llll}\text { P } & 2.49839 & -.24335 & -.30080\end{array}$

$\begin{array}{llll}\mathrm{H} & -.34408 & 1.25036 & 1.76009\end{array}$

$\begin{array}{lllll}\text { O } & 2.84980 & .90321 & -1.35701\end{array}$

C $1.90758 \quad 1.89212-1.86425$

$\begin{array}{lllll}\text { O } & 3.92292 & -.92693 & -.04299\end{array}$

C $4.67444-1.56716-1.09851$

$\begin{array}{llll}\text { O } & 1.71719 & -1.43892 & -1.02480\end{array}$

C $.49108-1.25514-1.79037$

$\begin{array}{lllll}\mathrm{H} & -.55109 & -.38904 & .96866\end{array}$

$\begin{array}{llll}\mathrm{H} & 1.69843 & -.38482 & 1.89934\end{array}$

$\begin{array}{lllll}\mathrm{H} & 1.60030 & 1.58369 & -2.86723\end{array}$

$\begin{array}{llll}\mathrm{H} & 2.46211 & 2.83043 & -1.92257\end{array}$

H $1.02585 \quad 1.98344-1.22067$

H $4.08787-2.37395-1.54402$

$\begin{array}{llll}\mathrm{H} & 4.95233 & -.83361 & -1.86012\end{array}$

H $\quad 5.57035-1.97047 \quad-.62492$

H $\quad-.09184 \quad-2.16572-1.64463$

H $\quad .76201-1.15998-2.84616$

$\begin{array}{llll}\mathrm{H} & -.07068 & -.37667 & -1.44754\end{array}$

$\begin{array}{llll}\mathrm{H} & 2.12495 & 1.30705 & 1.40661\end{array}$

\section{L. $\mathrm{Me}_{3} \mathrm{PCH}_{2}$ МeCHO}

\section{TS1 (B3L YP 6-31G*)}

$\mathrm{E}\left(\mathrm{B} 3 \mathrm{LYP} 6-31 \mathrm{G}^{*}\right)=-654.18826$

$\begin{array}{cccc}\mathrm{O} & 1.21464 & 1.50213 & 1.51125 \\ \mathrm{C} & 1.82740 & .57291 & .89779 \\ \mathrm{P} & -.92481 & .22325 & -.22398 \\ \mathrm{C} & .72030 & -.26811 & -.57883 \\ \mathrm{H} & 1.95077 & -.40377 & 1.41893 \\ \mathrm{H} & 1.04518 & .13612 & -1.54126 \\ \mathrm{C} & 3.07610 & .93366 & .09140 \\ \mathrm{H} & 3.47064 & .08408 & -.47847 \\ \mathrm{H} & 2.86091 & 1.76300 & -.59147 \\ \mathrm{H} & 3.84997 & 1.27524 & .79239 \\ \mathrm{H} & .85963 & -1.35180 & -.54023 \\ \mathrm{C} & -1.01090 & 2.03048 & -.41104 \\ \mathrm{H} & -1.99042 & 2.41541 & -.11155 \\ \mathrm{H} & -.21656 & 2.41525 & .24386 \\ \mathrm{H} & -.81337 & 2.30929 & -1.45082 \\ \mathrm{C} & -2.29466 & -.49407 & -1.22859 \\ \mathrm{H} & -3.26282 & -.08872 & -.91166 \\ \mathrm{H} & -2.13920 & -.26133 & -2.28632 \\ \mathrm{H} & -2.31007 & -1.58224 & -1.11074 \\ \mathrm{C} & -1.32149 & -.15980 & 1.51279 \\ \mathrm{H} & -2.33540 & .16776 & 1.76294\end{array}$




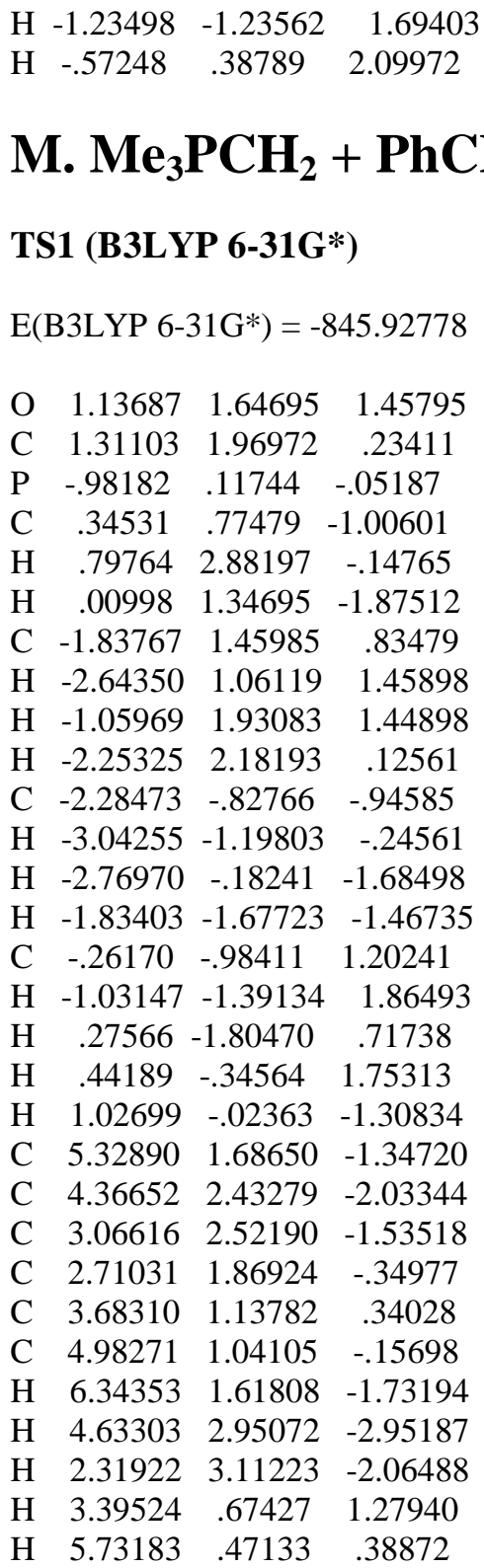

\section{N. $\mathrm{Me}_{3} \mathrm{PCHMe}+\mathrm{CH}_{2} \mathrm{O}$ O. $\mathrm{Me}_{3} \mathrm{PCHPh}+$ $\mathrm{CH}_{2} \mathrm{O}$} TS1 (B3LYP 6-31G*)

$\mathrm{E}\left(\mathrm{B} 3 \mathrm{LYP} 6-31 \mathrm{G}^{*}\right)=-654.17840$

$\begin{array}{cccc}\mathrm{O} & 1.06422 & 1.56685 & 1.74552 \\ \mathrm{C} & 1.64142 & 1.26333 & .67450 \\ \mathrm{P} & -1.08943 & .09845 & -.04291 \\ \mathrm{C} & .52571 & -.00764 & -.68076 \\ \mathrm{H} & 2.46351 & .51863 & .66495 \\ \mathrm{H} & 1.74869 & 2.01236 & -.13817 \\ \mathrm{C} & -1.57122 & 1.84508 & .18191 \\ \mathrm{H} & -2.59187 & 1.91963 & .56927 \\ \mathrm{H} & -.85154 & 2.26429 & .89619 \\ \mathrm{H} & -1.51186 & 2.38218 & -.76975 \\ \mathrm{C} & -2.49911 & -.67568 & -.96143\end{array}$

$\begin{array}{cccc}\mathrm{H} & -3.44896 & -.51678 & -.43750 \\ \mathrm{H} & -2.57120 & -.23802 & -1.96237 \\ \mathrm{H} & -2.32371 & -1.75090 & -1.06631 \\ \mathrm{C} & -1.07627 & -.66389 & 1.61056 \\ \mathrm{H} & -2.05986 & -.58958 & 2.08450 \\ \mathrm{H} & -.79468 & -1.71862 & 1.53187 \\ \mathrm{H} & -.32477 & -.11733 & 2.19319 \\ \mathrm{H} & .94057 & -.99874 & -.47913 \\ \mathrm{C} & .83922 & .52610 & -2.06677 \\ \mathrm{H} & 1.92368 & .67908 & -2.16268 \\ \mathrm{H} & .53529 & -.13032 & -2.89634 \\ \mathrm{H} & .37615 & 1.50669 & -2.24040\end{array}$

\section{TS1b (B3LYP 6-31G*)}

$$
\mathrm{E}\left(\mathrm{B} 3 \mathrm{LYP} 6-31 \mathrm{G}^{*}\right)=-654.17755
$$

$\begin{array}{cccc}\mathrm{O} & 1.06406 & 1.51867 & 1.79930 \\ \mathrm{C} & 1.66096 & 1.23857 & .73482 \\ \mathrm{P} & -1.06814 & .07358 & -.06975 \\ \mathrm{C} & .56242 & -.05265 & -.66480 \\ \mathrm{H} & 2.47971 & .49066 & .72559 \\ \mathrm{H} & 1.77585 & 1.99955 & -.06387 \\ \mathrm{H} & .67055 & .49381 & -1.60653 \\ \mathrm{C} & -1.51346 & 1.83793 & .04705 \\ \mathrm{H} & -2.55142 & 1.95857 & .37195 \\ \mathrm{H} & -.82506 & 2.27565 & .77990 \\ \mathrm{H} & -1.38373 & 2.32444 & -.92464 \\ \mathrm{C} & -2.45716 & -.73807 & -.99034 \\ \mathrm{H} & -3.41252 & -.60183 & -.46999 \\ \mathrm{H} & -2.53342 & -.30995 & -1.99498 \\ \mathrm{H} & -2.25709 & -1.81008 & -1.08656 \\ \mathrm{C} & -1.15440 & -.59157 & 1.62604 \\ \mathrm{H} & -2.16378 & -.47825 & 2.03346 \\ \mathrm{H} & -.88141 & -1.65064 & 1.63805 \\ \mathrm{H} & -.43280 & -.01038 & 2.21290 \\ \mathrm{C} & 1.24237 & -1.41402 & -.66339 \\ \mathrm{H} & 2.30402 & -1.28815 & -.91232 \\ \mathrm{H} & 1.21025 & -1.88083 & .32976 \\ \mathrm{H} & .83613 & -2.14330 & -1.38299\end{array}$

\section{TS1 (B3LYP 6-31G*)}

$\mathrm{E}\left(\mathrm{B} 3 \mathrm{LYP} 6-31 \mathrm{G}^{*}\right)=-845.92284$

$\begin{array}{cccc}\mathrm{O} & 1.00153 & 2.07428 & 1.10924 \\ \mathrm{C} & 1.61092 & 1.02190 & .72560 \\ \mathrm{P} & -1.08133 & .15216 & .12418 \\ \mathrm{C} & .57306 & -.05835 & -.49700 \\ \mathrm{H} & 1.79536 & .19810 & 1.45694 \\ \mathrm{C} & 1.17358 & -1.39957 & -.63014 \\ \mathrm{C} & 1.09414 & -2.37653 & .38391 \\ \mathrm{C} & 1.76276 & -3.59465 & .26894 \\ \mathrm{C} & 2.54674 & -3.86889 & -.85258\end{array}$

$\begin{array}{cccc}\mathrm{C} & 2.65090 & -2.90639 & -1.86018 \\ \mathrm{C} & 1.97373 & -1.69483 & -1.75195 \\ \mathrm{H} & 2.48504 & 1.10955 & .04144 \\ \mathrm{H} & .63533 & .52526 & -1.41857 \\ \mathrm{H} & .52367 & -2.17534 & 1.28699 \\ \mathrm{H} & 1.68085 & -4.32652 & 1.06882 \\ \mathrm{H} & 3.07508 & -4.81423 & -.93713 \\ \mathrm{H} & 3.26289 & -3.10048 & -2.73760 \\ \mathrm{H} & 2.06227 & -.95421 & -2.54393 \\ \mathrm{C} & -1.61816 & 1.80761 & -.40054 \\ \mathrm{H} & -2.58215 & 2.07098 & .04556 \\ \mathrm{H} & -.82610 & 2.47896 & -.04227 \\ \mathrm{H} & -1.69366 & 1.85447 & -1.49114 \\ \mathrm{C} & -2.29338 & -1.08912 & -.47348 \\ \mathrm{H} & -3.26962 & -.93867 & -.00002 \\ \mathrm{H} & -2.40146 & -1.00819 & -1.55898 \\ \mathrm{H} & -1.92803 & -2.09270 & -.23645 \\ \mathrm{C} & -1.18292 & .15255 & 1.94780 \\ \mathrm{H} & -2.20820 & .37935 & 2.25786 \\ \mathrm{H} & -.88764 & -.81205 & 2.36675 \\ \mathrm{H} & -.49328 & .94509 & 2.26630\end{array}$

TS1b (B3LYP 6-31G*)

$\mathrm{E}\left(\mathrm{B} 3 \mathrm{LYP} 6-31 \mathrm{G}^{*}\right)=-845.92098$

$\begin{array}{llll}\text { O } & 1.09674 & 1.34658 & 1.74975\end{array}$

$\begin{array}{llll}\text { C } & 1.60003 & 1.26247 & .59443\end{array}$

$\begin{array}{llll}\mathrm{P}-1.06233 & .10472 & -.02876\end{array}$

C $\quad .58476 \quad-.04076 \quad-.64589$

$\begin{array}{llll}\mathrm{H} & 2.55047 & .71545 & .43723\end{array}$

$\begin{array}{llll}\text { C } & 1.22037 & -1.38520 & -.59015\end{array}$

$\begin{array}{llll}\text { C } & 1.62725 & -1.94147 & .64116\end{array}$

$\begin{array}{llll}\text { C } & 2.26174 & -3.18182 & .69284\end{array}$

$\begin{array}{llll}\text { C } & 2.53064 & -3.89144 & -.47996\end{array}$

$\begin{array}{llll}\text { C } & 2.16289 & -3.34020 & -1.70938\end{array}$

$\begin{array}{llll}\text { C } & 1.51231 & -2.10799 & -1.76235\end{array}$

$\begin{array}{llll}\mathrm{H} & 1.49979 & 2.11515 & -.11369\end{array}$

$\begin{array}{llll}\mathrm{H} & .62206 & .40889 & -1.64448\end{array}$

$\begin{array}{llll}\mathrm{H} & 1.48372 & -1.36674 & 1.55180\end{array}$

H $\quad 2.56684 \quad-3.58615 \quad 1.65514$

$\begin{array}{llll}\mathrm{H} & 3.03210 & -4.85456 & -.43748\end{array}$

$\begin{array}{llll}\mathrm{H} & 2.37917 & -3.87318 & -2.63224\end{array}$

$\begin{array}{llll}\mathrm{H} & 1.22560 & -1.69068 & -2.72579\end{array}$

$\begin{array}{llll}\mathrm{C}-1.50307 & 1.86913 & .10665\end{array}$

$\begin{array}{lll}\mathrm{H}-2.54526 & 1.98198 & .42107\end{array}$

$\begin{array}{llll}\mathrm{H} & -.82621 & 2.28750 & .86009\end{array}$

$\begin{array}{llll}\mathrm{H} & -1.35926 & 2.37437 & -.85284\end{array}$

$\begin{array}{llll}\text { C } & -2.37783 & -.68896 & -1.04564\end{array}$

$\begin{array}{llll}\mathrm{H} & -3.36157 & -.57810 & -.57505\end{array}$

$\begin{array}{llll}\mathrm{H} & -2.40447 & -.23281 & -2.04016\end{array}$

H $-2.15162-1.75367 \quad-1.15791$

$\begin{array}{llll}\text { C }-1.22522 & -.59958 & 1.64063\end{array}$

$\begin{array}{llll}\mathrm{H} & -2.23600 & -.42083 & 2.02107\end{array}$

H $-1.02704-1.67408 \quad 1.61964$

$\begin{array}{llll}\mathrm{H} & -.48086 & -.08810 & 2.26039\end{array}$ 


\begin{tabular}{|c|c|c|c|c|c|c|c|}
\hline \multirow{4}{*}{\multicolumn{4}{|c|}{$\begin{array}{l}\text { P. } \mathrm{Me}_{3} \mathrm{PCHCOOMe}+ \\
\mathrm{CH}_{2} \mathrm{O}\end{array}$}} & $\mathrm{C}$ & -1.20679 & -0.73794 & 1.75584 \\
\hline & & & & $\mathrm{H}$ & -2.16648 & -0.44872 & 2.19743 \\
\hline & & & & $\mathrm{H}$ & -1.14517 & -1.82055 & 1.63909 \\
\hline & & & & $\mathrm{H}$ & -0.36613 & -0.36190 & 2.34289 \\
\hline \multirow{2}{*}{\multicolumn{4}{|c|}{ TS1 (B3LYP 6-31G*) }} & $\mathrm{C}$ & 1.11876 & -1.47846 & -0.61553 \\
\hline & & & & $\mathrm{O}$ & 2.26605 & -1.57753 & -1.32316 \\
\hline \multirow{2}{*}{\multicolumn{4}{|c|}{$\mathrm{E}\left(\mathrm{B} 3 \mathrm{LYP} 6-31 \mathrm{G}^{*}\right)=-842.75625$}} & $\mathrm{O}$ & 0.62064 & -2.42843 & -0.02626 \\
\hline & & & & $\mathrm{C}$ & 2.89150 & -2.86949 & -1.30372 \\
\hline $\mathrm{O}$ & .85035 & 1.69465 & 1.31687 & $\mathrm{H}$ & 3.79377 & -2.76466 & -1.90681 \\
\hline $\mathrm{C}$ & 1.57865 & .75927 & .76893 & $\mathrm{H}$ & 3.14500 & -3.15772 & -0.28042 \\
\hline $\mathrm{P}$ & -1.02455 & .13163 & .11652 & $\mathrm{H}$ & 2.22840 & -3.62649 & -1.73152 \\
\hline $\mathrm{C}$ & .68172 & -.00624 & -.49373 & & & & \\
\hline $\mathrm{H}$ & 1.85254 & -.11618 & 1.41249 & & & & \\
\hline $\mathrm{H}$ & 2.50353 & 1.06109 & .22385 & & & & \\
\hline $\mathrm{H}$ & .80099 & 63289 & -1.37080 & & & & \\
\hline $\mathrm{C}$ & -1.62209 & 1.83023 & -.15444 & & & & \\
\hline $\mathrm{H}$ & -2.57590 & 1.98317 & .36055 & & & & \\
\hline $\mathrm{H}$ & -.84013 & 2.47527 & .25822 & & & & \\
\hline $\mathrm{H}$ & -1.75889 & 2.00512 & -1.22674 & & & & \\
\hline $\mathrm{C}$ & -2.13193 & -.95593 & -.85918 & & & & \\
\hline $\mathrm{H}$ & -3.16671 & -.83472 & -.52157 & & & & \\
\hline $\mathrm{H}$ & -2.07698 & -.68702 & -1.91866 & & & & \\
\hline $\mathrm{H}$ & -1.81937 & -1.99500 & -.73715 & & & & \\
\hline $\mathrm{C}$ & -1.18985 & -.37722 & 1.85542 & & & & \\
\hline $\mathrm{H}$ & -2.24402 & -.36634 & 2.15045 & & & & \\
\hline $\mathrm{H}$ & -.78769 & -1.38866 & 1.95575 & & & & \\
\hline $\mathrm{H}$ & -.59762 & .33927 & 2.43078 & & & & \\
\hline $\mathrm{C}$ & 1.11160 & -1.40323 & -.71628 & & & & \\
\hline $\mathrm{O}$ & 2.21621 & -1.46314 & -1.49378 & & & & \\
\hline $\mathrm{O}$ & .62001 & -2.39071 & -.18085 & & & & \\
\hline $\mathrm{C}$ & 2.80742 & -2.76503 & -1.62785 & & & & \\
\hline $\mathrm{H}$ & 3.68345 & -2.62283 & -2.26141 & & & & \\
\hline $\mathrm{H}$ & 3.09958 & -3.15728 & -.65029 & & & & \\
\hline $\mathrm{H}$ & 2.10577 & -3.46223 & -2.09416 & & & & \\
\hline
\end{tabular}

\section{TS1 conformer with PCCO dihedral angle fixed at $\mathbf{3 0}^{\circ}$ (B3LYP 6-31G*)}

$\begin{array}{lrrr}\text { E(B3LYP 6-31G*) }=-842.75177 \\ \text { O } & 1.02984 & 1.08832 & 1.53820 \\ \text { C } & 1.51312 & 1.02753 & 0.32759 \\ \text { P } & -1.04190 & 0.04822 & 0.12400 \\ \text { C } & 0.60649 & -0.08492 & -0.63433 \\ \text { H } & 2.55690 & 0.65799 & 0.19340 \\ \text { H } & 1.41908 & 1.94640 & -0.31044 \\ \text { H } & 0.57739 & 0.29245 & -1.66216 \\ \text { C } & -1.52441 & 1.80090 & 0.22382 \\ \text { H } & -2.55788 & 1.88331 & 0.57454 \\ \text { H } & -0.82291 & 2.24677 & 0.93512 \\ \text { H } & -1.43755 & 2.27783 & -0.75684 \\ \text { C } & -2.25837 & -0.77270 & -0.97441 \\ \text { H } & -3.26406 & -0.70894 & -0.54608 \\ \text { H } & -2.26291 & -0.30013 & -1.96130 \\ \text { H } & -1.98190 & -1.82489 & -1.08322\end{array}$

